\title{
Experimental Studies of the Stimulated Brillouin Scattering Instability in the Saturated Regime
}

\author{
D.H. Froula
}

Doctor of Philosophy in Physics

\section{October 29, 2002}

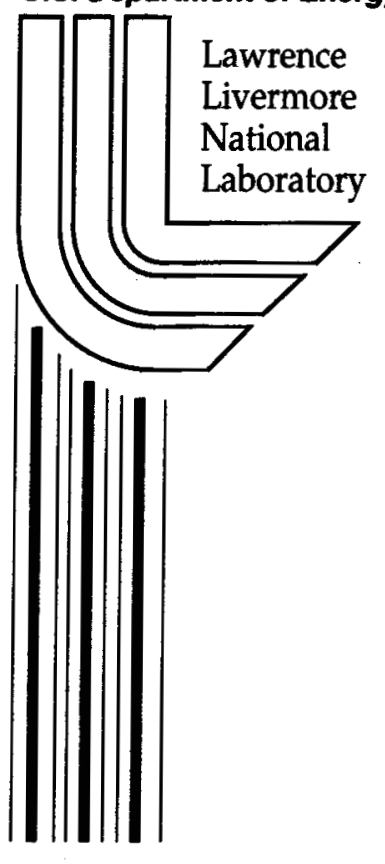




\section{DISCLAIMER}

This document was prepared as an account of work sponsored by an agency of the United States Government. Neither the United States Government nor the University of California nor any of their employees, makes any warranty, express or implied, or assumes any legal liability or responsibility for the accuracy, completeness, or usefulness of any information, apparatus, product, or process disclosed, or represents that its use would not infringe privately owned rights. Reference herein to any specific commercial product, process, or service by trade name, trademark, manufacturer, or otherwise, does not necessarily constitute or imply its endorsement, recommendation, or favoring by the United States Government or the University of California. The views and opinions of authors expressed herein do not necessarily state or reflect those of the United States Government or the University of California, and shall not be used for advertising or product endorsement purposes.

This work was performed under the auspices of the U. S. Department of Energy by the University of California, Lawrence Livermore National Laboratory under Contract No. W-7405-Eng-48.

This report has been reproduced directly from the best available copy.

Available electronically at http://www.doe.gov/bridge

Available for a processing fee to U.S. Department of Energy and its contractors in paper from

U.S. Department of Energy

Office of Scientific and Technical Information

P.O. Box 62

Oak Ridge, TN 37831-0062

Telephone: (865) 576-8401

Facsimile: (865) 576-5728

E-mail: reports@adonis.osti.gov

Available for the sale to the public from

U.S. Department of Commerce

National Technical Information Service 5285 Port Royal Road Springfield, VA 22161

Telephone: (800) 553-6847

Facsimile: (703) 605-6900

E-mail: orders@ntis.fedworld.gov

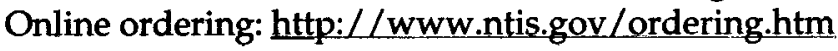

OR

Lawrence Livermore National Laboratory

Technical Information Department's Digital Library

http://www.llnl.gov/tid/Library.html 
Experimental studies of the stimulated Brillouin scattering instability in the saturated regime

by

Dustin Henry Froula

B.S.(California Polytechnic State University, San Luis Obispo) 1998

M.S. (University of California, Davis) 2000

A dissertation submitted in partial satisfaction of the requirements for the degree of

Doctor of Philosophy

in

Physics

in the

GRADUATE DIVISION

of the

UNIVERSITY OF CALIFORNIA, DAVIS

Approved:

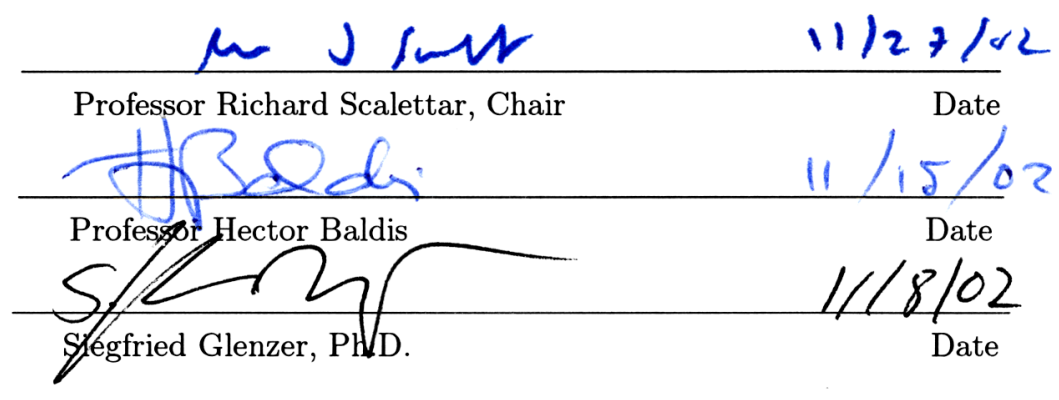

Committee in charge

Fall 2002 
Experimental studies of the stimulated Brillouin scattering instability in the saturated regime

Copyright 2002

by

Dustin Henry Froula 


\begin{abstract} saturated regime

by

Dustin Henry Froula

Doctor of Philosophy in Physics

University of California, Davis

Professor Richard Scalettar, Chair
\end{abstract}

Experimental studies of the stimulated Brillouin scattering instability in the

An experimental study of the stimulated Brillouin scattering (SBS) instability has investigated the effects of velocity gradients and kinetic effects on the saturation of ion-acoustic waves in a plasma. For intensities less than $\mathrm{I}<1.5 \times 10^{15} \mathrm{~W} \mathrm{~cm}^{-2}$, the SBS instability is moderated primarily by velocity gradients, and for intensities above this threshold, nonlinear trapping is invoked to saturate the instability. We report direct evidence of detuning of SBS by a velocity gradient which was achieved by directly measuring the frequency shift of the SBS driven acoustic wave relative to the local resonant acoustic frequency. Furthermore, a novel use of Thomson scattering has allowed us to gather direct evidence of kinetic effects associated with the SBS process. Specifically, a measured two-fold increase of the ion temperature has been linked with laser beam excitation of ion-acoustic waves to large amplitudes by the SBS instability. Ion-acoustic waves were excited to large amplitude with a $2 \omega$ 
1.2-ns long interaction beam with intensities up to $5 \times 10^{15} \mathrm{~W} \mathrm{~cm}^{-2}$. The local frequency, amplitude, and spatial range of these waves were measured with a $3 \omega$ 200ps Thomsonscattering probe beam. These detailed and accurate measurements in well-characterized plasma conditions allow for the first time a direct test of non-linear models of the saturation of SBS. The measured two-fold increase of the ion temperature and its correlation with SBS reflectivity measurements is the first quantitative evidence of hot ions created by ion trapping in laser plasmas.

$\mu \mathrm{sen} \quad 11 / 2+1 / 2$

Professor Richard Scalettar

Dissertation Committee Chair 
To my parents:

It was Grandpa Froula's interest in education that trickled down through my father and created an environment where knowledge is valued. Throughout my life it has been invaluable to have my father as a provider of ideas and a discusser of projects. His ability to educate and advise is truly the reason I am a scientist.

It is my mother who has taught me to see a problem as an opportunity to find a solution. Her support and encouragement has enabled me to bring many ideas to fruition. Her ability to teach me to look for the whole picture surrounding an issue has developed me as a person and as a scientist; I am lucky to have her keen insight into our world. 
To the educators in my life who supported "my" approach to learning while creating an environment that stimulated my curiosity and provided the necessary structure for success:

Ms. Sis Shaperio (1980)

Mr. Jim Harvey (1987)

The Dunn School (1990 - 1993)

Ms. Nancy Roome (1991)

Ms. Rose Knoles (1992)

Mr. Ralph Lowe (1983)

Dr. Ronald Brown (1997) 


\section{Contents}

List of Figures vi

List of Tables $\quad$ xii

1 Introduction $\quad 1$

1.1 Plasma physics . . . . . . . . . . . . . . . . . . . 1

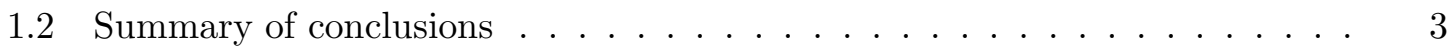

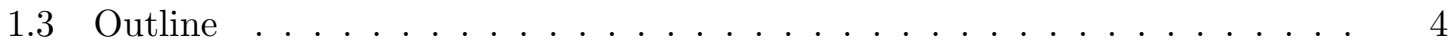

$\begin{array}{llr}\text { I General plasma theory } & 6\end{array}$

2 General Plasma Physics $\quad 7$

2.1 Kinetic theory . . . . . . . . . . . . . . . . . 7

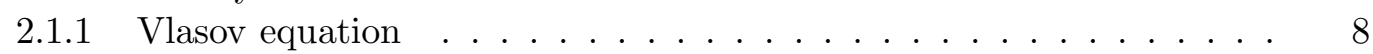

2.1 .2 Plasma dielectric function . . . . . . . . . . . . . . 8

2.2 Ion-acoustic wave dispersion relations . . . . . . . . . . . . . . 10

2.2.1 Single species dispersion relation . . . . . . . . . . . . . . . 11

2.2 .2 Multi-ion species dispersion relation . . . . . . . . . . . . . 12

2.3 Linear Landau damping . . . . . . . . . . . . . . . . . . . . . . . . . . . . 14

2.4 Non-linear Landau damping . . . . . . . . . . . . . . . . . . . . . . 15

2.5 Particle motion in Be-Au plasmas . . . . . . . . . . . . . . . . 16

3 Stimulated Brillouin scattering $\quad 20$

3.1 Electromagnetic and ion-acoustic wave interactions . . . . . . . . . . . 21

3.2 Driven acoustic waves (ponderomotive coupling) . . . . . . . . . . . 22

3.3 Stimulated Brillouin scattering reflectivity (linear theory) . . . . . . . . . 23

3.4 Saturation of the stimulated Brillouin scattering reflectivity by non-linear

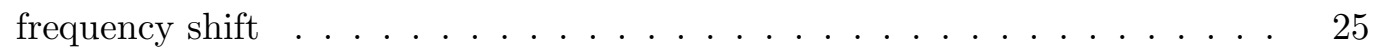

4 Thomson Scattering Diagnostic $\quad 29$

4.1 Thomson scattering parameter . . . . . . . . . . . . 31 
4.2 Thomson scattering theory $\ldots \ldots \ldots \ldots \ldots \ldots$

4.3 Multi-ion species Thomson scattering spectra . . . . . . . . . . . . 35

4.4 Scattering from stimulated Brillouin excited acoustic waves . . . . . . . 37

II Experiment $\quad 40$

5 Experimental Setup $\quad 41$

5.1 Trident Laser Facility . . . . . . . . . . . . . . . . . . . . . . . . 42

5.2 Overview of experimental configuration . . . . . . . . . . . . 42

5.2 .1 Beam transport . . . . . . . . . . . . . . . . 43

5.2 .2 Pulse shaping . . . . . . . . . . . . . . . . . . . 44

5.2 .3 Beam timing . . . . . . . . . . . . . . . . . . . 49

5.3 Instrumentation . . . . . . . . . . . . . . . . 51

5.3 .1 Spectrometer characterization . . . . . . . . . . . 51

5.4 Full aperture backscatter station configuration . . . . . . . . . . . 51

5.4 .1 Backscatter beam transport . . . . . . . . . . . . . 51

5.4 .2 Backscatter energy measurement . . . . . . . . . . . . 53

5.4 .3 Backscatter spectral measurements . . . . . . . . . . . . . . 53

5.5 Thomson-scattering instrumentation . . . . . . . . . . . . 56

5.5.1 "Thermal" Thomson scattering $\left(\mathrm{k}_{\perp}\right) \ldots \ldots \ldots 6$

5.5 .2 "Driven" Thomson scattering $\left(k_{\|}\right) \ldots \ldots \ldots \ldots$

6 Plasma Characterization $\quad \mathbf{5 9}$

6.1 Target configuration . . . . . . . . . . . . . . . . . 59

6.2 Electron density . . . . . . . . . . . . . . . . . . . 60

6.3 Average charge state $(\mathrm{Z}) \ldots \ldots \ldots \ldots$. . . . . . . . . . . . 61

6.4 Plasma electron temperature . . . . . . . . . . . . . . . 62

$6.4 .1 T_{e}$ normal to target surface . . . . . . . . . . . . 62

$6.4 .2 T_{e}$ parallel to the interaction beam axis . . . . . . . . . 63

6.5 Plasma ion temperature . . . . . . . . . . . . . . . . . . 64

6.6 Plasma flow parallel to target surface . . . . . . . . . . . . . . . 65

$\begin{array}{lll}\text { III Results and Discussion } & 67\end{array}$

7 The effects of velocity gradients on SBS $\quad 68$

7.1 Experimental setup . . . . . . . . . . . . . . . . . . . 69

7.2 Plasma characterization . . . . . . . . . . . . . . 71

7.3 Detuning by a velocity gradient . . . . . . . . . . . . . 75

7.4 Chapter summary . . . . . . . . . . . . . . . . . . . . 79 
8 Observation of ion heating by stimulated-Brillouin-scattered-driven ionacoustic waves

8.1 Thomson scattering on SBS driven ion-acoustic waves . . . . . . . . . 82

8.2 Evidence of kinetic effects . . . . . . . . . . . . . . . . . . 84

8.3 Summary . . . . . . . . . . . . . . . . . . . 89

$\begin{array}{ll}\text { IV Summary and conclusions } & 91\end{array}$

9 Summary $\quad 92$

9.1 Introduction . . . . . . . . . . . . . . . . . . . . . . . . . . . 92

9.1.1 Thomson scattering . . . . . . . . . . . . . . 94

9.2 Plasma characterization . . . . . . . . . . . . . . . . 97

9.2 .1 Plasma temperature . . . . . . . . . . . . . . . . . 97

9.2 .2 Plasma flow . . . . . . . . . . . . . . . . . . . . 99

9.2.3 Plasma electron density . . . . . . . . . . . . . . . . . 99

9.3 Saturated SBS regime . . . . . . . . . . . . . . . . . . . . 102

9.3 .1 Velocity gradient . . . . . . . . . . . . . . . . 103

9.3.2 Kinetic effects (Trapping) . . . . . . . . . . . . . . . 108

9.4 Summary . . . . . . . . . . . . . . . . . . . . . 111

10 Conclusions 113

$\begin{array}{ll}\text { Bibliography } & \mathbf{1 1 5}\end{array}$

$\begin{array}{lll}\text { V Appendix } & 123\end{array}$

A "Measurements of non-linear growth of ion-acoustic waves in two-ionspecies plasmas with Thomson scattering", D. H. Froula, et al., Physical Reveiw Letters, 88 (2002)

B "Obervation of ion heating by stimulated-Brillouin-scattered-driven ionacoustic waves using Thomson scattering", D. H. Froula, et al., Physics of Plasmas, 9(10) (2002)

C "Stimulated Brillouin scattering (SBS) in the saturated regime", D. H. Froula, et al., Physics of Plasmas Special Issue, Accepted for publication May (2003) (APS-DPP Invited)

D "Direct observation of stimulated Brillouin scattering (SBS) detuning by a velocity gradient", D. H. Froula, et al., Physical Review Letters, submitted (2002) 


\section{List of Figures}

2.1 The magnitude and phase of the susceptibility for each species is shown for both the slow and fast modes by the amplitude and angle of the corresponding arrow. In the slow mode, the Be ions screen (top left $\operatorname{Re}\left(\chi_{B e}\right)>0$ ) the motion of the $\mathrm{Au}$ ions (middle left $\left.\operatorname{Re}\left(\chi_{A u}\right)<0\right)$. The $\mathrm{Au}$ ions participate very little in the fast mode (middle right $\left|\chi_{B e}\right|<<1$ ). In both modes the electrons have a screening effect (bottom row). There is a small imaginary component in the suceptibilities of the fast mode, therefore, the mode is weakly damped (right side). The slow mode has significant contributions along the imaginary axis resulting in large damping. . . . . . . . . . . . . . . .

2.2 Normalized Landau damping, $\nu_{a} / \omega$, for the fast mode (dashed line) and the slow mode (solid line), as a function of the ion temperature for a fixed electron temperature, $T_{e}=600 \mathrm{eV}$. The concentration of $\mathrm{Au}$ was $10 \%$. The shaded region corresponds to the range of ion temperatures in the present experiment where the slow mode is more heavily damped than the fast mode.

3.1 (1) Incident light scatters from density fluctuations. (2) The scattered light beats with incident light. (3) The ponderomotive force drives density fluctuations with a wavelength equal to the beating wavelength. . . . . . . . .

3.2 (a) Calculations for SBS reflectivity as a function of Au concentration in Be plasmas. Linear theory predicts nearly $100 \%$ (open circles). Our nonlinear model (dark squares) predicts a $10 \%$ level of SBS. (b) As more Au ions are added to a Be plasma, phase velocity of the SBS driven wave decreases (right axis, dashed lines); more Be ions can be trapped, and the detuning parameter $(\eta)$ increases (left axis, solid lines). Two sets of curves are shown for $\mathrm{T}_{i}=400 \mathrm{eV}$ (upper curve) and $\mathrm{T}_{i}=250 \mathrm{eV}$ (lower curves). . . . . .

4.1 (color). Non-collective Thomson scattering (left) reveals a spectrum (right plot) that is a function of the velcity of the electrons. . . . . . . . . . .

4.2 (color). Light scattered from low frequency fluctuations reveals two peaks corresponding to co- and counter-propagating ion-acoustic waves. . . . . . . 32

4.3 The wave vector of the fluctuations are determined by momentum $\left(\mathbf{k}_{\mathbf{t s}}=\mathbf{k}_{\mathbf{s}}+\mathbf{k}\right)$ and energy $\left(\omega_{t s}=\omega_{s}+\omega\right)$ conservation. . . . . . . . . . . 
4.4 (a) The theoretical form factor is plotted for $20 \%$ variations in electron temperature, (b) and ion temperature while all other parameters are held fixed.

4.5 The ratio between the amplitude of the light scattered from the fast mode to the amplitude of the light scattered from the fast mode is plotted as a function of ion temperature for a fixed electron temperature, $\mathrm{T}_{e}=600 \mathrm{eV}$. The shaded area represents the experimental region. . . . . . . . . . .

4.6 A Thomson scattering spectrum is shown for a shot in which the red-shifted peak has been excited by SBS. The blue-shifted peaks are fit with the standard multi-ion-species form factor allowing the measure to the ion temperature, $\mathrm{T}_{i}=475 \mathrm{eV} \ldots \ldots \ldots \ldots \ldots \ldots$

5.1 Schematic of the experimental setup. . . . . . . . . . . . . .

5.2 Beam Transport is shown for both the interaction and heater beams, A and B respectively. . . . . . . . . . . . . . . .

5.3 The beam delivery is shown for the probe beam (Beam $\mathrm{C}) . \ldots \ldots \ldots$

5.4 The east view of the target chamber is shown providing an overview of the probe beam transport to target chamber center. The collection optics for both Thomson-scattering diagnostics are shown. . . . . . . . . . . . . .

5.5 An image of the focal spot of beam A taken on shot 5279 with a $75 \mu \mathrm{m}$ circle superimposed. In this image, low level, large area wings that account for about $1 / 3$ of the total energy are visible. . . . . . . . . . . . .

5.6 (color). Trident's driver laser uses a conventional master oscillator power amplifier architecture. Drive (A \& B beams) and probe ( $\mathrm{C}$ beam) pulses can be amplified sequentially through the front end before being routed to separate disk amplifier chains. If a short pulse is desired for beam C, the 200 ps pulse from the oscillator can be picked off before the pulse stacker. . . .

5.7 (a) One element was selected; the relative timing between beams $\mathrm{A}$ and $\mathrm{B}$ are measured at TCC. (b) Elements A1, A13 and beam C were selected; the relative timing between elements $\mathrm{A} 1$ and $\mathrm{A} 13$ was measured to be $2.34 \mathrm{~ns}$; this indicates that there is 180 ps between elements. The timing between element A1 and C was 1.17. The heater beam path (beam B) was blocked. These results are from campaign $2 \ldots \ldots \ldots \ldots$

5.8 A Ne spectra was measured with each spectrometer (except the $1 / 4$ meter) to calculate the instrument function and the dispersion. (a) $1 / 2$-meter $\mathrm{dts}$ (b) 1-meter dts (c) 1.5-meter tts (d) instrument function for one Ne-line from the 1-meter sbs spectrometer (e) 1-meter sbs (f) The 1/4-meter spectrometer was calabrated using two laser lines at $632 \mathrm{~nm}$ and $527 \mathrm{~nm}$. . . . . . . . . 52

5.9 FABS block diagram. . . . . . . . . . . . . . . . . . . . 54

5.10 A representative diode trace is shown for a Au shot with an interaction beam energy of $190 \mathrm{~J}$ (shot 050701069). The integrated signals are roughly equal corresponding to a $4 \%$ reflectivity. 
5.11 (a) The experimental setup is shown with three beams intersecting the Thomson volume at the target chamber center. Light is collected by two $\mathrm{f} / 5 \mathrm{col}$ lection lenses. (b) A vector diagram is shown where one Thomson diagnostic observes the $2 \mathbf{k}_{\mathbf{o}}$ wave vector propagating parallel to the interaction beam and the second Thomson diagnostic observes a wave vector perpendicular to the first. . . . . . . . . . . . . . . . . . . . . .

6.1 (a) The measured SRS spectrum is measured at $\mathrm{x}=400$ microns from the target surface. The peak frequency is $665 \mathrm{~nm}$. The line out is averaged over a $50 \mathrm{ps}$ region, $500 \mathrm{ps}$ after the interaction beam is turned on. (b) The electron density profile is plotted as a function of distance from the plasma surface, $x$. A LASNEX calculation is shown. . . . . . . . . . . . . .

6.2 Thomson-scattering spectra for three different $\mathrm{Au}$ concentrations averaged over $\sim 25$ ps are shown. (a) $1 \% \mathrm{Au}$ (b) $5 \% \mathrm{Au}$ (c) $10 \% \mathrm{Au}$. The solid line represents the best fit theoretical multi-ion-species form factor. . . . . . . .

6.3 (a) Gold Thomson-scattering streak data are shown for three separate distances from the target. (b) A 50 ps lineout is shown for the above Au streak data. (c) The electron temperature is plotted as a function of the distance from the target surface at a time of $1.4 \mathrm{~ns} . \ldots \ldots \ldots$. . . . . . . .

6.4 The electron temperature profile parallel to the interaction beam axis is shown to be constant. A best fit line is plotted. . . . . . . . . . . . . .

6.5 Light scattered from ion-acoustic waves counter-propagating to the interaction beam axis allow the measure of a velocity gradient along the interaction beam axis. The blue-shifted light is collected by the Driven Thomson Scattering Diagnostic. A Mach 1 flow is measured at the edge of the plasma. . .

7.1 (a) A diagram detailing the ion-acoustic wave-vectors probed is shown in the top left corner. (b) A thermal Thomson-scattering spectrum $\left(\mathrm{k}_{\perp}\right)$ and (d) a driven Thomson-scattering spectrum $\left(\mathrm{k}_{\|}\right)$are shown. (c) The experimental setup is shown. . . . . . . . . . . . . . . . .

7.2 (a) A constant electron temperature, $T_{e}=450 \mathrm{eV}$, is measured along the interaction beam axis. (b) Representative Thomson-scattering spectra taken from the spectra in Fig. 7.1b where light is scattered from ion-acoustic fluctuations propagating perpendicular to the interaction beam $\left(\mathbf{k}_{\perp}\right)$. (c) Thomson-scattering data are shown collected by the diagnostic observing ion-acoustic waves propagating parallel to the interaction beam $\left(\mathbf{k}_{\|}\right)$. The spectra (points) is shown below averaged over $50 \mathrm{ps}$. The theoretical form factor (solid line) is fit to the blue-shifted light using a velocity flow $\frac{v}{v_{t h}}=0.7$ and the electron temperature measured with the first Thomson-scattering diagnostic $\left(T_{e}=450 \mathrm{eV}\right)$. The shaded region was optically filtered by $\mathrm{x} 100$. 
7.3 (color). Intensity spectra at $\mathrm{z}=(\mathrm{a})-375 \mu \mathrm{m}$, (b) $-250 \mu \mathrm{m}$, and (c) $-125 \mu \mathrm{m}$ show a velocity gradient. Furthermore, the frequency of the SBS driven ionacoustic wave is unchanged. The shaded area has been filtered by a factor of 10. (d) The wavelength shift for red-shifted (solid squares) and the blueshifted (open circles) intensity peaks are plotted. The blue-shifted intensity peaks reveal a Mach 1 flow parallel to the interaction beam (right axis). A $\mathrm{pF} 3 \mathrm{~d}$ simulation is shown where large acoustic waves (color scale $=\delta \mathrm{n} / \mathrm{n}_{e}$ ) are driven in the plateau region. . . . . . . . . . . . . . .

7.4 (color). (a) The intensity of the interaction beam is plotted 70 ps into the pF3d simulation. (b) The ion-acoustic waves are large in the front of the plasma. (c) The measured amplitude of the excited ion-acoustic wave (squares) is plotted as a function of the position along the interaction beam. The amplitude profile given by pF3d is also plotted (solid). . . . . . . . . .

7.5 Ths SBS spectra is plotted as a function of intensity. The peak intensity corresponds to light scattered from the velocity plateau; a tail towards the fundamental frequency corresponds to light scattered from ion-acoustic waves in the front of the plasma. . . . . . . . . . . . . . . . .

8.1 (a) Streaked Thomson spectra for a Be plasma with a 10\% Au mixture are shown for a shot without the interaction beam (undriven) and shots with interaction beam intensities of $5 \times 10^{15} \mathrm{~W} \mathrm{~cm}^{-2}$ and $7 \times 10^{15} \mathrm{~W} \mathrm{~cm}^{-2}$ (driven). (b) Line outs show the increasing amplitude of the red-shifted light. The blueshifted peaks are fit with the standard multi-ion-species form factor showing an increase in ion temperature from $250 \mathrm{eV}$ to $475 \mathrm{eV}$. The data was collected using the driven Thomson scattering diagnostic described in Sec. 5.5 and the dts2 spectrometer (see Table 5.3). . . . . . . . . . . . . . . .

8.2 The blue-shifted (thermal) part of the Thomson-scattering spectra for a $10 \%$ $\mathrm{Au}$ mixture shows a shot without the interaction beam (triangles) and a shot with an interaction beam intensity of $6 \times 10^{15} \mathrm{~W} \mathrm{~cm}^{-2}$ (squares) resulting in an increase in ion temperature from 250 to $475 \mathrm{eV} \ldots \ldots$. . . . . . . .

8.3 The ratio of $T_{i} / T_{e}$ as a function of the normalized ion-wave amplitude shows evidance of the generation of hot ions and ion trapping. The ion-wave amplitude is normalized to the thermal ion-wave amplitude. Data for Be plasmas with $5 \% \mathrm{Au}$ (circles) and $10 \% \mathrm{Au}$ (squares) show a factor of two increase in ion temperature. The gray area represents calculated results from a simple energy balance between energy deposition due to SBS, and a free-streaming heat flux. . . . . . . . . . . . . . . . . . .

8.4 The SBS reflectivity has been measured to decrease for Be plasmas with increasing Au concentration (diamonds). This trend is reproduced by our non-linear SBS theory (shaded area). Linear calculations are also shown (black line) . . . . . . . . . . . . . . . . . . . 
8.5 (a) The results of a PIC sumulation of SBS show a phase diagram where trapping can clearly be seen to repeat at the spatial frequency of the driven ion-acoustic wave. (b) The Be distribution function evolves from an intial Maxwellian (dashed line). After 60 ps (solid line), a plateau at the phase velocity and a tail of hot ions is seen. . . . . . . . . . . . .

9.1 (a) The driven Thomson scattering spectra provides a measure of both the plasma flow along the axis of the interaction beam $\left(-\mathbf{k}_{\|}\right)$, the frequency of the SBS driven acoustic wave $\left(\mathbf{k}_{\|}\right)$, and the relative amplitude of the SBS driven ion-acoustic waves. This data is representative of a point at $\mathrm{z}=-375 \mu \mathrm{m}$ and $\mathrm{x}=400 \mu \mathrm{m}$ with an interaction beam intensity of $\mathrm{I}=1.5 \times 10^{15} \mathrm{~W} \mathrm{~cm}^{-2}$. (b) Our thermal Thomson-scattering spectra allow us to measure the electron temperature by observing ion-acoustic waves propagating perpendicular $\left(\mathrm{k}_{\perp}\right)$ to the driven ion-acoustic waves $\left(\mathrm{k}_{\|}\right) . \ldots \ldots \ldots \ldots$

9.2 The sound speed (right axis) and therefore the electron temperature (left axis) were measured to be a constant $\mathrm{T}_{e}=450 \mathrm{eV}$ along the interaction beam axis, $\mathrm{x}=400 \mu \mathrm{m}$ from the surface of the target. The line is a best fit. .

9.3 The frequency shift of the peak intensity scattered from thermal $\left(-\mathbf{k}_{\|}\right)$ionacoustic waves allows us to measure a velocity profile along the interaction beam axis. A LASNEX simulation using a 1-mm disk target reproduces the velcocity profile. . . . . . . . . . . . . . .

9.4 (a) The SRS spectra is measured at $\mathrm{x}=400 \mu \mathrm{m}$ from the target surface. A normalized linear gain calculation is fit to the data (line) to obtain the density. (b) The electron density calculated from the SRS spectra are plotted as a function of the distance from the target surface. LASNEX calculations are plotted (line) . . . . . . . . . . . . . . . . . . .

9.5 The ion-acoustic wave amplitude and the SBS reflectivity are plotted as a function of the intensity of the interaction beam. The target was moved to probe ion waves at $z=-375 \mu \mathrm{m}$. The plasma length along the interaction beam was $\mathrm{L}=1-\mathrm{mm} . \ldots \ldots \ldots$. . . . . . . . . . . . .

9.6 The wavelength shift of the peak intensity for the down shifted (solid squares) and up-shifted (open circles) Thomson-scattering peaks are plotted. The upshifted intensity peaks reveal a Mach 1 flow parallel to the interaction beam (right axis). The data are plotted on top of a pF3d simulation where ionacoustic waves are driven to large amplitude in the front of the velocity plateau. 105

9.7 The measured amplitude of the excited ion-acoustic wave $\left(\frac{\delta n}{n_{e}}\right)$ is plotted as a function of the position along the interaction beam. The calculated amplitude profile from pF3d (solid line) after averaging over a $70 \mu \mathrm{m}$ area similar to the experiment is shown. . . . . . . . . . . . . . . . 107

9.8 The (a) SBS reflectivity and (b) normalized ion-wave amplitude is plotted as a function of plasma length along the interaction beam axis. . . . . . . . . 108 
9.9 The ratio of $\mathrm{T}_{e} / T_{i}$ as a function of the normalized ion-wave amplitude shows evidence of the generation of hot ions and ion trapping. The ion-wave amplitude is normalized to the thermal ion-wave amplitude. Data for Be plasmas with $5 \% \mathrm{Au}$ (circles) and 10\% $\mathrm{Au}$ (squares) show a factor of two increase in ion temperature. The gray area represents calculated results from a simple energy balance between energy deposition due to SBS, and a free-streaming heat flux. . . . . . . . . . . . . . . . . . . 


\section{List of Tables}

5.1 Laser Beam Parameters . . . . . . . . . . . . . . . . . . . .

5.2 The relative beam timings are shown for two Trident campaigns. In campaign 1, elements 6-13 where used. In Campaign 2, elements 5-11 where selected. All numbers are in picoseconds. . . . . . . . . . . . . . . . . . . 50

5.3 The spectrometer characteristics are tabulated above. . . . . . . . . . . . 51 


\section{Acknowledgments}

I would like to thank my technical advisor Siegfried Glenzer for his support, ideas, and guidance throughout my Ph.D. studies. I appreciate the endless hours Laurent Divol spent working me through the theoretical concepts in this thesis and in physics in general. I would like to thank Hector Baldis and Richard Scalettar for making it possible for me to study at Lawrence Livermore National Laboratory. Without the dedication of the Trident Laser Crew (R. Johnson, R. Gibson, T. Hurry, R. Gonzales, N. Okamoto, F. Archuleta, S. Letzring, and R. Perea) through their late nights, extra shots, and knowledge of experimental setups these experiments would not have succeeded. I would like to acknowledge financial support from the Student Employee Graduate Research Fellowship Program. This work was partially supported by LDRD 01-ERD-107 and was performed under the auspices of the U.S. Department of Energy by the Lawrence Livermore National laboratory under contract No. W-7405-ENG-48. 


\section{Chapter 1}

\section{Introduction}

\subsection{Plasma physics}

A plasma is a quasineutral gas of electrons and ions that act in a collective manner. Although $98 \%$ of the matter in the universe is in the plasma state, there are few examples of plasmas in our world. Because of the charged nature of the particles in a plasma, plasmas exhibit fascinating collective behaviors that are not seen in normal gases. Among these are a large variety of wave processes that are due to the long range of the Coulomb force that correlate distant charged particles $[1,2]$.

Over the last 50 years, plasma physicists have used linearized equations to describe plasmas with surprising accuracy. Nature is inherently a non-linear system due to the interweaving of other systems that interfere, cooperate, or compete, making it impossible to write separable linear equations that work over a large range of parameter space. Computers have recently become tools for simulating aspects of these complex systems, allowing scientists to develop models where non-linear processes dominate the experimental 
observables, for example, laser plasma interactions.

The interaction between an incident laser beam and a plasma, can produce a scattered electromagnetic wave in a process called stimulated Brillouin scattering. The SBS instability results from the resonant coupling of an intense laser pulse, a scattered light wave, and an ion-acoustic wave. The coupling between these three waves makes SBS inherently a non-linear process. Linear theory predicts that ion-acoustic waves will be driven by an incident electromagnetic wave to large amplitude where it can backscatter $100 \%$ of the incident energy. Experiments have shown that SBS driven ion-acoustic waves will saturate far below this point, therefore, indicating a need for a non-linear model which will limit the backscatter reflectivity $[3,4,5,6]$. Previous theories have suggested nonlinear saturation mechanisms of ion-acoustic waves through two-ion decay, trapping, and wave breaking $[3,7]$. In order to model recent experiments [8] a non-linear secondary decay process was suggested, and models used an unphysical clamp on the amplitude of the ion-acoustic wave to limit the backscatter reflectivity. This is a verification that non-linear mechanisms must be added to models. Furthermore, theory has long predicted that velocity gradients will create a mismatch in the parametric SBS instability therefore, reducing the amplitude of the ion-acoustic waves [cite].

In this thesis, we will experimentally investigate the ability for hydrodynamics (velocity gradients) and non-linear kinetic effects (trapping) to saturate ion-acoustic waves in a plasma. We use a new technique that measures the relative damping of two ionacoustic modes to determine the ion temperature with high accuracy. We measure up to a factor of two increase in the ion temperature when ion-acoustic waves are excited by 
SBS. This measurement of the ion temperature, and its correlation with SBS, is the first direct quantitative evidence of hot ions created by trapping in laser produced plasmas. Furthermore, we report the first direct evidence of detuning of SBS by a velocity gradient, which was achieved by directly measuring the frequency shift of the SBS driven acoustic wave relative to the local resonant acoustic frequency.

\subsection{Summary of conclusions}

Two saturation process that are important in limiting the SBS reflectivity have been observed and are discussed in this thesis: saturation due to velocity gradients, and direct quantitative evidence of hot ions created by trapping. We will present data that indicate that both the ion-acoustic wave and the SBS reflectivity saturate for intensities above $\mathrm{I}=1.5 \times 10^{15} \mathrm{~W} \mathrm{~cm}^{-2}$. It is clear that non-linear effects have saturated the SBS instability. The findings have been summarized in four publications which have been included as appendices.

We have measured an increase in ion temperature as a function of the SBS excited ion-acoustic wave amplitude. As the interaction beam excites ion-acoustic waves, the ion temperature in the plasma was measured to increase, while we found no change in the electron temperature. This direct observation of an increase in ion temperature is an indication of ion trapping. The driven ion waves accelerate ions through non-linear trapping creating hot ions. After thermalization, this will increase the average ion temperature which we have measured using Thomson scattering. It is clear in these experiments that driving ionacoustic waves to large amplitude transfers energy into the ions (kinetics). In our range of 
experimental parameters the population of Be ions near the phase velocity of the acoustic wave is large enough so that trapping effects are a plausible saturation mechanism. Trapping induces a modification of the dispersion relation for the SBS acoustic wave, creating a non-linear frequency shift $[7,9]$. This non-linear frequency shift detunes the SBS three wave coupling therefore, saturating the ion-acoustic wave.

We have directly investigated the detuning process using two Thomson-scattering diagnostics that allow a direct measure of the shift in the frequency of the local SBS driven ion-acoustic wave relative to the beat frequency of the incident and reflected light. Using this novel technique we present the first measurement of the actual detuning of the SBS driven ion-acoustic wave while simultaneously measuring the local velocity flow. Velocity gradients shift the local resonant frequency, detuning the SBS instability and reducing the threshold for non-linear effects. But clearly once the interaction beam intensity exceeds an intensity of $1.5 \times 10^{15} \mathrm{~W} \mathrm{~cm}^{-2}$ previously discussed kinetic effects (trapping) play an important role in saturating SBS. Preliminary modeling is consistent with experiments indicating that both velocity gradients and trapping effects are critical.

\subsection{Outline}

The thesis is broken into four parts: General plasma theory, Experiments, Results and discussions, and Summary and conclusions. Part I discusses a kinetic approach to plasma physics developing the necessary background to understand stimulated Brillouin scattering and the theoretical underpinning of Thomson scattering. In Part II the laser configuration, the diagnostics, and the plasma characterization are described. Part III is 
split into two chapters that discuss our measurements of the detuning of the SBS instability. Chapter 7 reports on the effects of velocity gradients while Chapter 8 discusses our measurements of kinetic effects. The results are summarized in Part IV. 


\section{Part I}

General plasma theory 


\section{Chapter 2}

\section{General Plasma Physics}

In this chapter we will investigate the ion motion within a plasma by calculating the evolution of the velocity distribution functions. This can be described by the Vlasov equation when collisions are ignored. The Vlasov equation will allow us to develop a plasma dielectric function which can be used to determine the particle motion and specifically the ion-acoustic wave dispersion relation.

\subsection{Kinetic theory}

A kinetic description of a plasma allows one to consider each specie's velocity distribution independently. The one-dimensional particle distribution function is the probability density associated with an ensemble of particles and can be assumed to be a MaxwellianBoltzmann:

$$
f_{o}^{\alpha}=\left(\frac{m_{\alpha}}{2 \pi k_{b} T_{\alpha}}\right)^{1 / 2} e^{-\frac{v^{2}}{2 v_{\alpha}^{2}}}
$$


where $k_{b}$ is the Boltzmann constant, $v_{\alpha} \equiv\left(\frac{k_{b} T_{\alpha}}{m_{\alpha}}\right)^{1 / 2}, \mathrm{~T}_{\alpha}$, and $m_{\alpha}$ are the respective species temperature and mass. $\alpha$ denotes the particle species (e.g. electrons, ions). The kinetic description will allow us to calculate the general ion-acoustic dispersion relation, the damping rates, and will provide insight into the electron and ion particle motion.

\subsubsection{Vlasov equation}

The Vlasov equation $[10,2,11]$, in conjunction with Maxwell's equations, provides a complete description of a collisionless plasma. The Vlasov equation is simply the continuity equation describing the evolution of the particle distribution function, $f(\mathbf{x}, \mathbf{v}, t)$ in phase space,

$$
\frac{\partial f}{\partial t}+\frac{\partial}{\partial \mathbf{x}} \cdot(\mathbf{v} f)+\frac{\partial}{\partial \mathbf{v}} \cdot(\mathbf{a} f)=0
$$

Newton's Second Law and the Lorentz Force Law can be solved for the acceleration,

$$
\mathbf{a}=\frac{q}{m}(\mathbf{E}+\mathbf{v} \times \mathbf{B}),
$$

where $\mathrm{m}$ and $\mathrm{q}$ are the mass and charge of the particles; $\mathbf{E}$ and $\mathbf{B}$ are the electric and magnetic fields. Combining Eq. (2.2) and Eq. (2.3) one arrives at the Vlasov equation,

$$
\frac{\partial f}{\partial t}+\mathbf{v} \cdot \frac{\partial f}{\partial \mathbf{x}}+\frac{q}{m}(\mathbf{E}+\mathbf{v} \times \mathbf{B}) \cdot \frac{\partial f}{\partial \mathbf{v}}=0
$$

From the Vlasov equation, we can use the Poisson equation to relate the electric fields to the density and determine the plasma dielectric function.

\subsubsection{Plasma dielectric function}

The Vlasov equation in conjunction with Poisson's equation can be used to derive the plasma dielectric function which describes the response of the plasma to small per- 
turbations in the electric field. Assuming a distribution function for each particle in the form,

$$
f(\mathbf{x}, \mathbf{v}, t)=f_{o}(\mathbf{v})+f_{1}(\mathbf{x}, t),
$$

where $f_{o}$ is the distribution function (Eq. 2.1) and $f_{1}$ is a first order perturbation around the equilibrium distribution, $f_{1} \propto e^{-i(\mathbf{k} \cdot \mathbf{x}-\omega t)}$, the Poisson equation can take the form,

$$
\varepsilon_{o} \nabla \cdot \mathbf{E}_{\mathbf{1}}=-e n_{e} \int f_{1}^{e} d \mathbf{v}+\sum_{\alpha} Z_{\alpha} q_{\alpha} n_{\alpha} \int f_{1}^{\alpha} d \mathbf{v}
$$

where $Z_{\alpha}$ is the average charge state, $n_{\alpha}$ is the density of the $\alpha$-ion species, and $\mathbf{E}_{\mathbf{1}} \propto$ $e^{-i(\mathbf{k} \cdot \mathbf{x}-\omega t)}$ is the electric field resulting from the small perturbation in the distribution function.

Letting the external magnetic and electric fields be zero, $\mathbf{B}_{o}=\mathbf{E}_{o}=0$, and reducing the dimensions to one for simplicity, the Vlasov equation (Eq. 2.4) can be written,

$$
\frac{\partial f_{1}^{\alpha}}{\partial t}+v \frac{\partial f_{1}^{\alpha}}{\partial x}+\frac{q E_{1}}{m} \frac{\partial f_{o}^{\alpha}}{\partial v}=0
$$

Solving this equation for the first order perturbation in the particle distribution function,

$$
f_{1}^{\alpha}=\frac{i Z_{\alpha} E_{1}^{\alpha}}{m_{\alpha}} \frac{\frac{\partial f_{o}^{\alpha}}{\partial v}}{\omega-k v}
$$

and substituting into the Poisson equation (Eq. 2.6) the plasma dielectric function can be defined:

$$
\varepsilon \equiv 1-\frac{e^{2} n_{e}}{\varepsilon_{o} m_{e} k^{2}} \int \frac{\frac{\partial f_{o}^{e}}{\partial v}}{v-\frac{\omega}{k}} d v-\sum_{\alpha} \frac{e^{2} Z_{\alpha}^{2} n_{\alpha}}{\varepsilon_{o} m_{\alpha} k^{2}} \int \frac{\frac{\partial f_{o}^{\alpha}}{\partial v}}{v-\frac{\omega}{k}} d v .
$$

As can be demonstrated by the Poisson equation, the field generated by a density perturbation scales as the inverse of the dielectric function. Therefore, the roots of Eq. 2.9 describe the normal modes of the plasmas (i.e. resonant responses). 
The first and second non-constant terms in Eq. 2.9 are defined as the electron, $\chi_{e}$, and ion, $\chi_{\alpha}$, susceptibilities respectively. The particle susceptibility has a singularity at the phase velocity $\left(v_{\phi} \equiv \frac{\omega}{k}\right)$. In general the particle susceptibility can be solved numerically [12]. In the special case when the phase velocity is large and there is weak damping Landau showed that the integral can be solved in the complex plane using a contour that follows the real axis with a small semicircle around the pole [13]. In this case the equation for the susceptibility becomes,

$$
\chi_{\alpha}=-\frac{\omega_{\alpha}^{2}}{k^{2}}\left[P \int_{-\infty}^{+\infty} \frac{\frac{\partial f_{o}^{\alpha}}{\partial v}}{v-v_{\phi}} d v+\left.i \pi \frac{\partial f_{o}^{\alpha}}{\partial v}\right|_{v=\omega / k}\right]
$$

where $\omega_{\alpha}^{2}=\frac{e^{2} Z_{\alpha}^{2} n_{\alpha}}{\varepsilon_{o} m_{\alpha}}$ is the plasma frequency of the $\alpha$-species; P denotes the Cauchy principal value. The contribution of the different particles to each mode is apparent through the calculation of the respective species susceptibilities. The real and imaginary parts of the susceptibility are treated separately in the following sections.

\subsection{Ion-acoustic wave dispersion relations}

The ion-acoustic wave dispersion relation describes the low frequency collective effects in a plasma. The thermal velocity $\left(v_{t h} \equiv \sqrt{\frac{k_{b} T}{m}}\right)$ of a specific species specifies how it participates in the collective effects. When a species has a large thermal velocity, it tends to screen electric potentials that are setup by slower species. We can therefore, categorize the species into two limiting sets separated by the phase velocity of the wave. The susceptibility (Eq. 2.10) of a species with a thermal velocity much greater than the wave's phase velocity 
$\left(v_{t h}>>v_{\phi}\right)$ can be Taylor expanded around $\frac{v_{\phi}}{v} \approx 0$ giving,

$$
\left.R \epsilon\left(\chi_{\alpha}\right)\right|_{v_{\phi}<<v_{t h}}=\frac{1}{k^{2} \lambda_{\alpha}^{2}}
$$

where $\lambda_{\alpha}^{2}=\frac{\varepsilon_{o} T_{\alpha}}{Z_{\alpha}^{2} e^{2} n_{\alpha}}$ is the Debye length for a given species.

In a plasma, waves will be supported by the species moving much slower than the phase velocity. In this case, $v_{\phi}>>v_{t h}$ so we can Taylor expand Eq. 2.10 around $\frac{v}{v_{\phi}} \approx 0$ giving,

$$
R \epsilon\left(\chi_{\alpha}\right)=-\frac{\omega_{\alpha}^{2}}{k^{2}}\left[\frac{1}{v_{\phi}^{2}}\left(1+\frac{3 v_{\alpha}^{2}}{v_{\phi}^{2}}\right)\right]_{v_{\phi}>>v_{t h}} .
$$

\subsubsection{Single species dispersion relation}

The normal modes of a plasma can be determined by solving for the roots of the plasma dielectric function,

$$
\varepsilon=1+\chi_{e}+\chi_{i}=0
$$

Because we are interested in the low frequency ion-acoustic waves, we can assume that the electrons are moving much faster than the wave $\left(v_{e}>>v_{\phi}\right)$ and therefore move to screen the electric potential of the slower ion motion. We can then substitute the screening solution (Eq. 2.11 ) for the electrons and the wave-like solution (Eq. 2.12) for the ions into Eq. 2.13,

$$
1-\frac{1}{k^{2} \lambda_{e}^{2}}+\frac{\omega_{i}^{2}}{k^{2}}\left[\frac{k^{2}}{\omega^{2}}\left(1+3 v_{i}^{2} \frac{k^{2}}{\omega^{2}}\right)\right]=0
$$

The ion susceptibility, $\chi_{i}$, is much greater than one $\left(\frac{\omega_{i}^{2}}{\omega^{2}}>>1\right)$ therefore, the single species ion-acoustic dispersion relation becomes,

$$
c_{s}^{2} \equiv \frac{\omega^{2}}{k^{2}}=\frac{k_{b} T_{e}}{M_{i}}\left[\frac{Z}{1+\left(k \lambda_{e}\right)^{2}}+\frac{3 T_{e}}{T_{i}}\right] .
$$


Where $c_{s}$ is defined as the sound speed in a plasma. It is apparent that the sound speed of the ion-acoustic wave scales, to first order, as the product of the charge state and the electron temperature. Thomson scattering experimentally measures the sound speed of waves and therefore, providing a direct measure of the electron temperature when the charge state is known.

\subsubsection{Multi-ion species dispersion relation}

We will now look at a plasma that consists of multi-ion species. Specifically the use $\mathrm{Au}-\mathrm{Be}$ ions to derive a dispersion relation will help understand physical trends in experiments which used a mixture of $\mathrm{Au}$-Be to make a novel ion temperature measurement.

For a plasma with $\mathrm{Au}$ and Be ions, there are two weakly damped acoustic solutions to Eq. 2.9. Because a Au-Be plasma has two phase velocities (i.e. two modes), we can separate the species into three limiting sets, as compared with the two used for the singleion species case. There are species that have a thermal velocity which is less than both of the phase velocities, species that have a thermal velocity between the phase velocities, and species that have a thermal velocity greater than both of the phase velocities. Once again the normal modes of the plasma can be determined by solving Eq. 2.9,

$$
\varepsilon=1+\chi_{e}+\chi_{B e}+\chi_{A u}=0 .
$$

\section{"Fast" ion-acoustic wave dispersion relation}

For the mode that has the larger phase velocity (defined as the "fast" mode), both ion-species oscillate in a wave-like behavior while the electrons work to screen the ion motion $\left(v_{B e}<<v_{\phi}<<v_{e}\right)$. Therefore, the roots of the dielectric function (Eq.2.16) can be 
found by substituting Eq. 2.11 for the screening electrons and Eq. 2.12 for the Au and Be ions into Eq. 2.16,

$$
R \epsilon(\varepsilon)=1+\frac{1}{k^{2} \lambda_{e}^{2}}+\frac{\omega_{B e}^{2}}{\omega^{2}}+\frac{\omega_{A u}^{2}}{\omega^{2}}=0
$$

The Au susceptibility is much smaller than the Be susceptibility due to the respective masses $\left(\frac{m_{B e}}{m_{A u}}<<1\right)$ and therefore can be ignored. The Be susceptibility is much greater than one, leading to a dispersion relation for the fast ion-acoustic mode which is similar to the single ion species case (Eq. 2.15),

$$
\left(\frac{\omega}{k}\right)_{f a s t}^{2}=\frac{Z_{B e} k_{b} T_{e}}{m_{B e}}+\ldots
$$

Once again the phase velocity of the wave scales with the electron temperature.

\section{"Slow" ion-acoustic wave dispersion relation}

In the mode with the smaller phase velocity (defined to be the "slow" mode), the $\mathrm{Au}$ ions oscillate, while both the electrons and Be-ions screen the potential created by the $\mathrm{Au}$ motion. In this case, solving for the root of the dielectric function (Eq. 2.16) a phase velocity between the thermal velocities of the $\mathrm{Be}$ and $\mathrm{Au}$ ions $\left(v_{A u}<<v_{\phi}<<v_{B e}\right)$ is used. Therefore, the screening susceptibility, Eq. 2.11, is used for both the Be ions and the electrons; the wave-like solution, Eq. 2.12, is used for the slow Au motion. Here we keep only the first order terms and note that both ion susceptibilities are greater than one,

$$
\left(\frac{\omega}{k}\right)_{\text {slow }}^{2}=\frac{Z_{A u} k_{b} T_{e}}{m_{A u}}\left[\frac{n_{B e}}{n_{A u}}+\frac{T_{e}}{T_{B e}} \frac{n_{B e}}{n_{A u}}\right]^{-1}
$$

This is an important result for our experiments as it allows us to measure the average charge state of the Au ions. As in the single species case, the phase velocity of the modes are proportional to the product of the electron temperature and the individual species charge 
state. Therefore, if the charge state of one of the species is known (as is the case with Be because it is relatively easily fully ionized) then the electron temperature is determined by the fast mode allowing for the charge state of the second species to be determined by the phase velocity of the slow mode.

\subsection{Linear Landau damping}

In 1947 Landau predicted that even in a collisionless plasma there would be damping of electrostatic waves due to acceleration of particles near the phase velocity. Landau pointed out that a correct treatment of the Vlasov equation gives a finite imaginary part the susceptibility [11]. For an electrostatic wave propagating through a plasma, the majority of the particles are not moving at the waves phase velocity but merely oscillate in the field taking no energy from the electrostatic wave. Landau damping is caused by particles moving at velocities near the phase velocity of an electrostatic wave. These particles feel a nearly constant field and therefore, can be efficiently accelerated. This transfer of energy from the wave to the particles damps the electrostatic wave. There are two main conditions for Landau damping to be effective:

(1) there must be sufficient number of particles at the phase velocity,

(2) there must be more particles moving slightly slower than the phase velocity than slightly faster than the phase velocity, namely the distribution function must have a negative slope around the phase velocity, $\frac{d f_{o}\left(v_{\phi}\right)}{d v}<0$. 
Landau damping accelerates the particles moving slightly slower than the phase velocity, while decelerating the particles move slightly faster than the phase velocity. If there are more particles (around the phase velocity) moving slower than the phase velocity, then there will be a net energy transfer from the field into the particles and the wave will be damped. Conversely if there are more particles moving faster than the phase velocity then there will be a net energy gain in the electrostatic wave and the wave will grow. The normalized damping rate $\left(\frac{\nu_{i}}{\omega}\right)$ can be calculated from the imaginary part of the dielectric function (Eq. 2.9) which gives,

$$
\frac{\nu_{i}}{\omega}=\sqrt{\frac{\pi}{8}}\left(\frac{Z T_{e}}{T_{i}}\right)^{3 / 2} e^{-\frac{Z T_{e}}{2 T_{i}}}
$$

for the ion species and,

$$
\frac{\nu_{e}}{\omega}=\sqrt{\frac{\pi}{8}}\left(\frac{Z m_{e}}{m_{i}}\right)^{1 / 2} \approx 1.5 \times 10^{-2} \sqrt{Z / A}
$$

for the electrons, where $\mathrm{A}$ is the atomic number. For a typical fully ionized Be plasma $\left(T_{e} / T_{i}=3\right)$, the Landau damping is $5 \%$. In Sec. 4.3 we will plot the damping rates for our Be-Au experiments which has led to a unique measure of the ion temperature in our plasmas.

\section{$2.4 \quad$ Non-linear Landau damping}

When ion-acoustic waves grow to large amplitude, Landau damping becomes nonlinear and particles begin to be trapped in the electrostatic potential of the ion waves. These trapped particles are accelerated along with the ion-acoustic wave as they oscillate (bounce) 
from one side of the potential to the other. Trapping modifies the dispersion relation which tends to slow the ion-acoustic wave. If there are a reasonable number of collisions, these accelerated particles will collide with untrapped particles increasing the ion temperature of the plasma.

\subsection{Particle motion in Be-Au plasmas}

The relative motion of the different particles to each mode is apparent through the calculation of the respective species susceptibilities which is shown in Fig. 2.1. The relative motion of the particles is given by the sign of the real vector component of each specie's susceptibility $\left(\operatorname{R\epsilon }\left(\chi_{\alpha}\right)\right)$. For the slow mode (left column), both the Be and electron species have susceptibilities with a real component (Eq. 2.10) opposing that of the Au species, therefore, both the electron and Be ion motion screen the Au ion fluctuations.

The right column of Fig. 2.1 shows the respective motion of the species to the fast mode. Here both the $\mathrm{Be}$ and $\mathrm{Au}$ ions participate in the wave (i.e. both have real vector components in the same direction, Eq. 2.12), while the electron motion screen the fields set up by the ions.

For both the slow and fast modes, the damping is controlled by the Be ions as the $\mathrm{Au}$ ions are moving much slower than the phase velocities of either mode $\left(v_{A u}<<v_{\phi}\right)$ and therefore, there are not enough $\mathrm{Au}$ ions to participate in damping the wave, condition (1) in Sec. 2.3. There is a small amount of damping due to the electrons which is not sensitive to the electron or ion temperature (Eq. 2.21).

Figure 2.2 shows the normalized Landau damping for the fast and slow modes as 


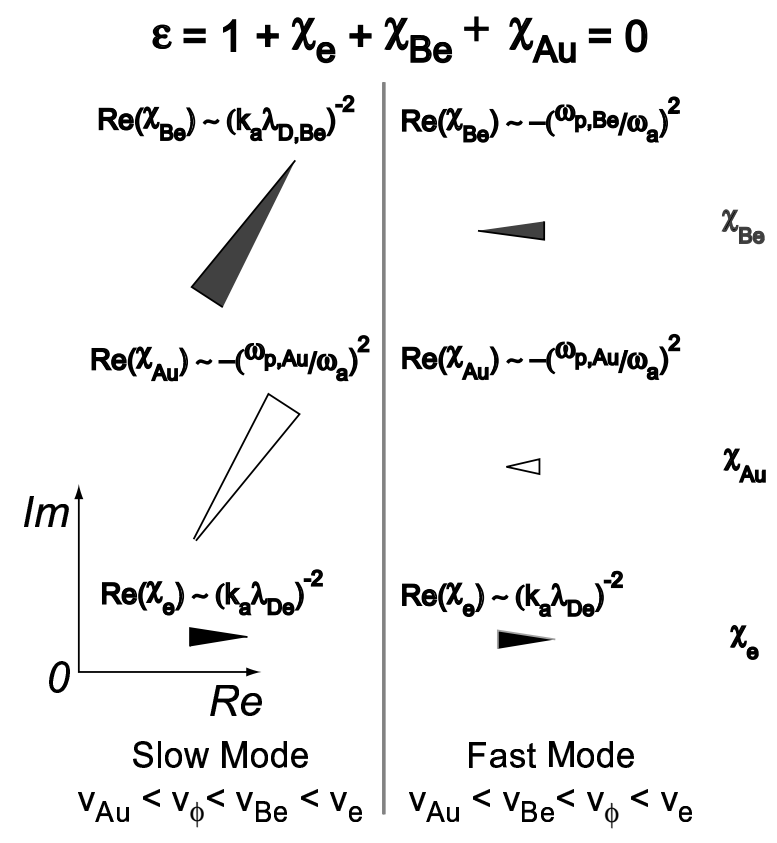

Figure 2.1: The magnitude and phase of the susceptibility for each species is shown for both the slow and fast modes by the amplitude and angle of the corresponding arrow. In the slow mode, the Be ions screen (top left $\operatorname{Re}\left(\chi_{B e}\right)>0$ ) the motion of the $\mathrm{Au}$ ions (middle left $\left.\operatorname{Re}\left(\chi_{A u}\right)<0\right)$. The $\mathrm{Au}$ ions participate very little in the fast mode (middle right $\left|\chi_{B e}\right|<<1$ ). In both modes the electrons have a screening effect (bottom row). There is a small imaginary component in the suceptibilities of the fast mode, therefore, the mode is weakly damped (right side). The slow mode has significant contributions along the imaginary axis resulting in large damping. 
a function of ion temperature. Notice as the ion temperature increases the damping of the two modes reacts qualitatively different; the damping of the fast mode increases while the damping of the slow mode decreases. This can be explained physically by looking at the details of where the phase velocity is in regards to the Be ion velocity distribution function. As the ion temperature increases, the distribution function of the Be ions broadens. Therefore, the number of particles at the phase velocity of the fast mode available to participate in damping increases and the damping of the fast mode goes up (condition 1 in Sec. 2.3). This is in contrast to the slow mode where the phase velocity is in the bulk of the distribution function, therefore, the number of particles at the phase velocity of the mode stays relatively constant. Around the phase velocity of the slow mode, the proportion of fast ions increases more rapidly than the number of slow ions therefore, decreasing the Landau damping (condition 2 in Sec. 2.3). 


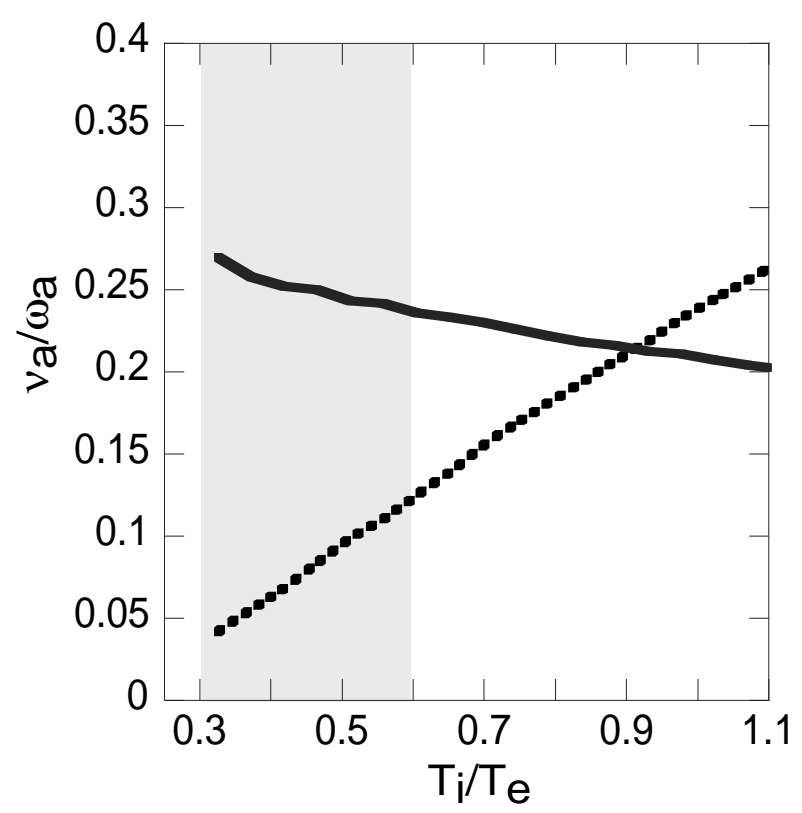

Figure 2.2: Normalized Landau damping, $\nu_{a} / \omega$, for the fast mode (dashed line) and the slow mode (solid line), as a function of the ion temperature for a fixed electron temperature, $T_{e}=600 \mathrm{eV}$. The concentration of Au was $10 \%$. The shaded region corresponds to the range of ion temperatures in the present experiment where the slow mode is more heavily damped than the fast mode. 


\section{Chapter 3}

\section{Stimulated Brillouin scattering}

In this chapter we will develop a three-wave model describing light interacting with a plasma. We will see that the ponderomotive force created by the beating of an incident and scattered light wave can drive ion-acoustic waves to large amplitude where a non-linear model is needed. We propose a non-linear frequency shift as a possible saturation mechanism.

The stimulated Brillouin scattering (SBS) instability results from the resonant coupling of the incident electromagnetic wave with an ion-acoustic wave and a back-scattered electromagnetic wave. The beating of the incident and scattered wave creates a ponderomotive force which resonantly drives the acoustic wave. In turn, the growing ion-acoustic wave Bragg scatters the incident wave, causing the ion waves to grow from thermal noise to large amplitude $[3,4,8,5,6]$. 


\subsection{Electromagnetic and ion-acoustic wave interactions}

An electromagnetic wave incident on a plasma will interact directly with the electrons which in turn set up an electric-potential that will interact with the ions. It is therefore through the electrons that the light wave indirectly couples to the ions. The electromagnetic wave equation for light propagating in a plasma can be derived from Maxwell equations assuming a constant background density of electrons,

$$
\left[\frac{\partial^{2}}{\partial t^{2}}-c^{2} \frac{\partial^{2}}{\partial x^{2}}+\omega_{p_{e}}^{2}\right] E(x, t)=0
$$

This equation can then be linearized by expanding the electric field and density,

$$
\begin{aligned}
& E(x, t)=E_{o}(x, t)+E_{s}(x, t), \\
& n(x, t)=n_{e}+\delta n(x, t),
\end{aligned}
$$

where $\mathrm{n}_{e}$ is the background neutral density and $\delta \mathrm{n}$ is a small first order perturbation around this density. Substituting these linearized equations into the electromagnetic wave equation (Eq. 3.1) keeping only first order terms and assuming $E_{s}$ is small compared to $E_{o}$ one gets,

$$
\left[\frac{\partial^{2}}{\partial t^{2}}-c^{2} \frac{\partial^{2}}{\partial x^{2}}+\omega_{p_{e}}^{2}\right] E_{s}(x, t)=-\omega_{p_{e}}^{2} \frac{\delta n}{n_{e}} E_{o}
$$

The fields can further be expressed as a product of a slow $(\tilde{E}(x, t))$ and fast $\left(e^{-i(k x-\omega t)}\right)$ oscillation:

$$
\begin{aligned}
& E_{o}=\tilde{E}_{o}(x, t) e^{-i\left(k_{o} x-\omega_{o} t\right)} \\
& E_{s}=\tilde{E}_{s}(x, t) e^{-i\left(-k_{s} x-\omega_{s} t\right)} \\
& \delta n=\tilde{\delta n}(x, t) e^{-i(k x-\omega t)} .
\end{aligned}
$$


Substituting these equations into the linearized wave equation (Eq. 3.4), while keeping only the first order low frequency terms, two coupled linearized equation describing the interaction between the incident light waves and ion-acoustic waves in a plasma is expressed,

$$
\begin{aligned}
& {\left[\frac{\partial}{\partial t}-c \frac{\partial}{\partial x}\right] \tilde{E}_{s}=i \frac{\omega_{p_{e}}^{2}}{2 \omega_{s}} \frac{\tilde{\delta n}}{n_{e}} \tilde{E}_{o}} \\
& {\left[\frac{\partial}{\partial t}+c \frac{\partial}{\partial x}\right] \tilde{E}_{o}=i \frac{\omega_{p_{e}}^{2}}{2 \omega_{s}} \frac{\tilde{\delta n}}{n_{e}} \tilde{E}_{s}}
\end{aligned}
$$

where $\tilde{E}_{o}$ and $\tilde{E}_{s}$ are the low frequency components of the electric field, E.

\subsection{Driven acoustic waves (ponderomotive coupling)}

The product between the incident and reflected light waves drives ion-acoustic fluctuations from an initial thermal level to large amplitudes. Electrons oscillating parallel to an electromagnetic field gradient, produced by the product of the incident and reflected light waves, move farther in the half-cycle when they are traveling from a strong electric field region to a weak electric field region, therefore, there is a net drift [2]. When the electromagnetic field gradient resonantly couples with the ion-acoustic fluctuations, then the electrons drift from regions of low electron density to regions of high electron density therefore, increasing the amplitude of the resonant ion-acoustic wave.

A wave equation that couples an electromagnetic field to a density fluctuation in a plasma can be derived through fluid equations to be [10],

$$
\left[\frac{\partial^{2}}{\partial t^{2}}+\nu_{L}-c_{s}^{2} \nabla^{2}\right] \frac{\delta n}{n_{e}}=\frac{Z e^{2}}{2 m_{i} m_{e} \omega_{o}^{2}} \nabla^{2}|E|^{2}
$$

where $\nu_{L}$ is the linear Landau damping (Section 2.3). Expressing the fields as a product of 
slow and fast oscillations (Eqs. 3.5-3.7) one finds,

$$
\left[\frac{\partial}{\partial t}+c_{s} \frac{\partial}{\partial x}+\nu_{L}\right] \frac{\tilde{\delta n}}{n_{e}}=i \frac{Z n_{e} e^{2} k^{2}}{4 m_{i} m_{e} \omega_{o}^{2} \omega}\left(\tilde{E}_{o} \tilde{E}_{s}\right)
$$

From Eq. 3.11 the coupling between the product of the incident and reflected light and the ion-acoustic wave is apparent. If either the incident or scattered electric field are increased, the amplitude of the acoustic wave grows. We can now use these coupled wave equations to calculate the SBS reflectivity from a plasma.

\subsection{Stimulated Brillouin scattering reflectivity (linear the- ory)}

An electromagnetic wave propagating through a plasma will scatter from density fluctuations. The light scattered from ion-acoustic waves will beat creating a ponderomotive force pushing electrons from low density regions of the acoustic wave to regions of high density. This in turn increases the amplitude of the acoustic wave which will increase the scattered light. The increased amplitude of the scattered electromagnetic wave will increase the ponderomotive force on the electrons and the process will repeat provided the acoustic wave remains resonant [Fig. 3.1].

The light reflected from the plasma $\left(\mathrm{r} \equiv\left(\frac{E_{s}(0)}{E_{o}(0)}\right)^{2}\right)$ can be calculated using a linear three wave model (Eqs. 3.8, 3.9, and 3.11). If we assume that the light waves have been driven into a steady state, Eqs. 3.8 and 3.9 become,

$$
\begin{aligned}
& -c_{s} \frac{\partial \tilde{E}_{s}}{\partial x}=i \frac{e^{2} n_{e}}{2 \epsilon_{o} m_{e} \omega_{o}} \frac{\tilde{\delta n}}{n_{e}} \tilde{E}_{o} \\
& c_{s} \frac{\partial \tilde{E}_{o}}{\partial x}=i \frac{e^{2} n_{e}}{2 \epsilon_{o} m_{e} \omega_{o}} \frac{\tilde{\delta n}}{n_{e}} \tilde{E}_{s}
\end{aligned}
$$




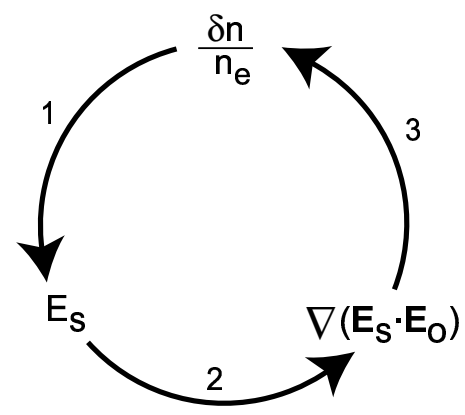

Figure 3.1: (1) Incident light scatters from density fluctuations. (2) The scattered light beats with incident light. (3) The ponderomotive force drives density fluctuations with a wavelength equal to the beating wavelength.

It can be shown that in the large damping limit $\left(\partial_{t} E_{s}-c_{s} \partial_{x} E_{s}\right)$ can be neglected and Eq. 3.11 will reduce to,

$$
\nu_{L} \frac{\tilde{\delta n}}{n_{e}}=\frac{i Z e^{2} k^{2}}{4 m_{i} m_{e} \omega_{o}^{2} \omega}\left(\tilde{E}_{o} \tilde{E}_{s}\right)
$$

If we further assume that there is no pump depletion, $\tilde{E}_{o}$ is a constant, we can easily solve this system of equations for the light backscattered along the incident beam between $\mathrm{z}=0$ and $\mathrm{z}=\mathrm{L}$,

$$
r \equiv\left(\frac{E_{s}(0)}{E_{o}}\right)^{2}=r(z=0) e^{2 G}
$$

where $\mathrm{L}$ is the length in which SBS is resonant and $\mathrm{G}$ is the linear gain,

$$
G \equiv \frac{\omega_{i}^{2} L}{4 c_{s}^{2}}\left(\frac{\omega}{\nu_{L}}\right)\left(\frac{e \tilde{E}_{o}}{m_{e} c \omega_{o}}\right)^{2}=4 \times 10^{-35}\left(\frac{Z n_{e}\left[\mathrm{~cm}^{-3}\right] L[\mu \mathrm{m}]}{T_{e}[e V]}\right)\left(\frac{\omega}{\nu_{L}}\right) I\left[W \mathrm{~cm}^{-3}\right]
$$

where $\omega$ is the ion-acoustic frequency and $c_{s}$ is the sound speed. The reflectivity at the far edge of the plasma $(\mathrm{r}(\mathrm{z}=0))$ can be calculated from thermal noise for our experimental conditions $\left(n_{e}=10^{20} \mathrm{~cm}^{-3}, \lambda_{o}=0.527 \mu \mathrm{m}, \Omega=3 \times 10^{-2}, \dot{L}=500 \mu \mathrm{m}\right)$ :

$$
r(z=0)=\frac{1}{2} r_{o}^{2} n_{e} \dot{L} \Omega \approx 6.2 \times 10^{-9}
$$


where $r_{o}=e^{2} / m c^{2}=2.8 \times 10^{-13} \mathrm{~cm}$ is the classical electron radius, $\dot{L}$ is the scale length in which the waves remain thermal and $\Omega$ is the solid angle of the collection optics.

We can estimate the value of the linear gain when the reflectivity reaches $100 \%$ by solving Eq. 3.15 for the gain; $G_{100 \%}=10$. This indicates that in order for linear theory to be valid the gain must be much less than $G_{100 \%}$. For our experimental conditions $\left(\frac{Z_{B e} T_{e}}{T_{i}}=10\right.$, $\lambda_{o}=0.527 \mu \mathrm{m}, \mathrm{L}=1000 \mu \mathrm{m}, \mathrm{I}=5 \times 10^{15} \mathrm{~W}-\mathrm{cm}^{-3}, \nu_{L} / \omega=0.05$ see Sect. 2.3), the gain is of order $\mathrm{G}=200$ therefore, it is clear a non-linear model must be used to saturate the ion-acoustic waves and limit the reflectivity.

\subsection{Saturation of the stimulated Brillouin scattering reflec- tivity by non-linear frequency shift}

In our range of experimental parameters $\left(6<Z_{B e} T_{e} / T_{i}<14\right)$ the population of Be ions near the phase velocity of the acoustic wave is large enough so that trapping effects are a plausible saturation mechanism. Trapping suppresses the ionic part of the linear Landau damping of the driven wave by flattening the distribution function around the phase velocity of the wave $[7,14]$; therefore, a lower limit for the residual damping, $\nu$, is the electronic Landau damping and a small collisional damping $\left(\nu_{e} / \omega_{s} \approx 0.015\right.$, see Sec. 2.3).

Another effect of the trapping is a modification of the dispersion relation for the 
SBS acoustic wave, creating a non-linear frequency shift, $\delta \Omega[7,9,15]$ :

$$
\begin{aligned}
\frac{\delta \Omega}{\omega} \approx & -\eta\left(\frac{\tilde{\delta n}}{n_{e}}\right)^{1 / 2} \equiv \\
& -\frac{f_{B e}}{\sqrt{2 \pi}}\left(v^{4}-v^{2}\right) e^{-v^{2} / 2} \sqrt{Z_{B e} \frac{T_{e}}{T_{i}}}\left(\frac{\tilde{\delta n}}{n_{e}}\right)^{1 / 2} ;
\end{aligned}
$$

where $\eta$ is the detuning parameter, $\omega$ is the SBS acoustic wave frequency, $f_{B e}$ is the fraction of Be ions in the plasma $\left(f_{B e}=0.9\right)$, and $v=v_{\phi} / v_{B e}, v_{B e}=\sqrt{\frac{k_{b} T_{i}}{M_{B e}}}$ is the thermal velocity of the Be ions. The phase velocity $\left(v_{\phi}\right)$ of the fast mode is given by the multi-ion-species dispersion relation $\left(v^{2}\right.$ is of order of $\left.Z_{B e} T_{e} / T_{i}\right)$.

This non-linear frequency shift detunes the resonant coupling between the interaction beam and the ion wave, therefore saturating the SBS instability. As the ion-acoustic waves are excited to large amplitude, ions are accelerated in the wave's potential well's therefore slowing the wave's phase velocity detuning the SBS resonance so that energy is no longer coupled in to these ion-acoustic waves by the incident laser.

Combining these effects in to the linear model developed in Section 3.2, one adds a damping term that is proportional to the non-linear frequency shift (Eq. 3.18) to Eq. $3.11[16]$ :

$$
\left(i \eta \sqrt{\frac{\tilde{\delta n}}{n_{e}}}+\nu\right) \frac{\delta \tilde{n}(z)}{n_{e}}=\frac{i Z e^{2} k^{2}}{4 \epsilon_{o} m_{i} m_{e} \omega_{o}^{2} \omega_{a}}\left(\tilde{E}_{o} \tilde{E}_{s}\right) .
$$

Balancing the two terms on the LHS of Eq. (3.19) shows that detuning becomes important for SBS saturation when $\delta n / n_{e} \approx \nu^{2} / \eta^{2} \simeq 0.1 \%$ for our parameters. Solving the system of equations [Eqs.(3.12-3.19)] between $\mathrm{z}=0$ and $\mathrm{z}=\mathrm{L}$ leads to:

$$
G_{S B S}(L)=\left[\ln \left|\frac{\tilde{\delta n}}{n_{e}}\right|+\frac{3 \eta^{2}}{2 \nu^{2}}\left|\frac{\tilde{\delta n}}{n_{e}}\right|\right]_{0}^{L},
$$


where $\mathrm{G}_{S B S}$ is the usual linear convective gain (see Sect. 3.3) calculated with the reduced damping discussed at the beginning of this section.

The logarithmic part of Eq. 3.20 is responsible for an exponential growth until detuning dominates the damping, leading to a polynomial growth in length. The SBS reflectivity for different fractions of Au is shown in (Fig. 3.2a). Parameters used in the model were selected to match the experiment $\left(\mathrm{L}=800 \mu \mathrm{m}, \mathrm{T}_{i} / \mathrm{T}_{e} \approx 1 / 2, \mathrm{I}_{0}=3 \times 10^{15} \mathrm{~W} \mathrm{~cm}^{-2}\right.$, $\left.\mathrm{n}_{e}=10^{20} \mathrm{~cm}^{-3}\right)$. The scaling with the fraction of $\mathrm{Au}$ ions comes mainly from a decreasing phase velocity of the fast mode when the fraction of $\mathrm{Au}$ ions is increased, which increases the number of ions at the phase velocity, resulting in a larger detuning parameter $(\eta)$, and therefore more efficient detuning, (i.e., the same acoustic wave amplitude creates a larger frequency shift) [Fig. 3.2]. 

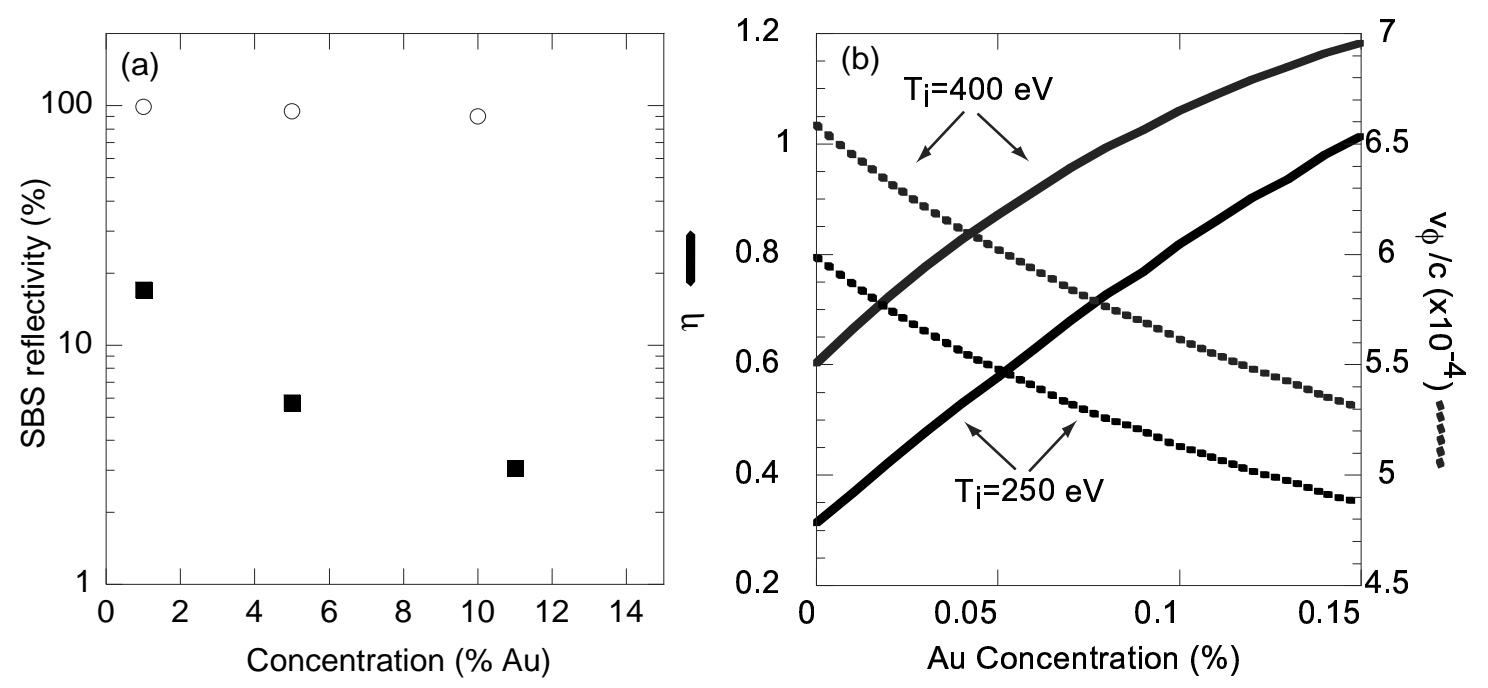

Figure 3.2: (a) Calculations for SBS reflectivity as a function of Au concentration in Be plasmas. Linear theory predicts nearly $100 \%$ (open circles). Our non-linear model (dark squares) predicts a $10 \%$ level of SBS. (b) As more Au ions are added to a Be plasma, phase velocity of the SBS driven wave decreases (right axis, dashed lines); more Be ions can be trapped, and the detuning parameter $(\eta)$ increases (left axis, solid lines). Two sets of curves are shown for $\mathrm{T}_{i}=400 \mathrm{eV}$ (upper curve) and $\mathrm{T}_{i}=250 \mathrm{eV}$ (lower curves). 


\section{Chapter 4}

\section{Thomson Scattering Diagnostic}

Thomson scattering is a powerful technique that allows one to directly measure the frequency and relative amplitude of oscillations in a plasma. From the measured frequency, the local sound speed, electron temperature [17], and particle velocity flow [18] can be determined. The amplitude of the fluctuation is proportional to the square root of the scattered intensity. In multi-species plasmas, the relative amplitude of the ion-acoustic waves allows an accurate determination of the ion temperature [19].

Through an investigation into the plasma dielectric function (Chapter 2) it is evident that a plasma will support density fluctuations which propagate with a specific dispersion. As we are primarily interested in low frequency fluctuations for our experiments, we will assume an ion-acoustic dispersion relation (Sec. 2.2).

Thomson scattering is the process in which electromagnetic waves are scattered by electrons [20]. For example, the electric field from an electromagnetic wave incident on a single electron will drive the electron to oscillate at the frequency of the incident 


\section{Non-collective \\ Thomson scattering $(\alpha<1)$}
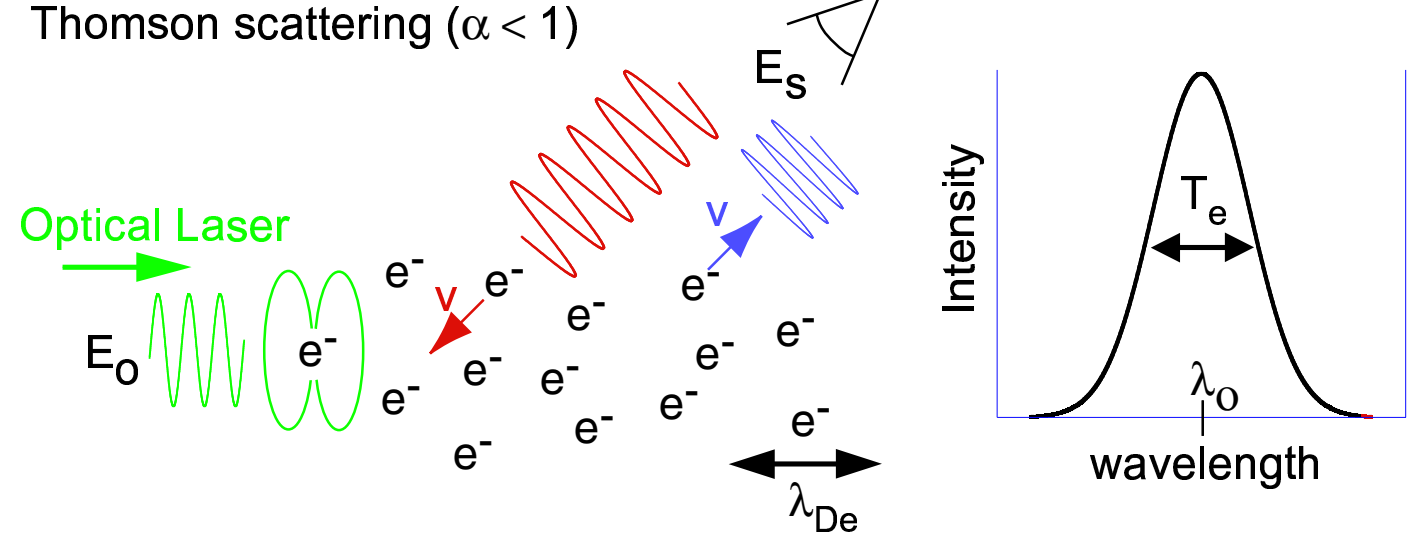

Figure 4.1: (color). Non-collective Thomson scattering (left) reveals a spectrum (right plot) that is a function of the velcity of the electrons.

wave [21]. The accelerating electron will radiate a scattered electromagnetic wave at the frequency of oscillation. If we then imagine that in addition to the oscillation due to the incident electromagnetic wave, the electron is moving at some velocity towards our detector, the radiated light will be Doppler shifted to higher wavelengths. The frequency shift of the scattered light is a measure of the velocity of the electron. If we further include an ensemble of electrons, our detector will measure light that is both red- (shifted to longer wavelengths) and blue-shifted (shifted to shorter wavelengths). By measuring the spectrum of the light scattered we can measure the electron velocity distribution function [Fig. 4.1].

Let us now look at an example where the plasma consists of both ions and electrons. We will setup our scattering geometry and the measured frequency window to allow for the observation of collective Thomson scattering from low frequency fluctuations in the plasma. In this case, we are observing light scattered from electrons that are screening the slow ion-acoustic motion. The motion of the ions, and consequently the electrons, are governed 
by the ion-acoustic dispersion relation (Eq. 2.15),

$$
c_{s}^{2}=\frac{k_{b} T_{e}}{M_{i}}\left[\frac{Z}{1+\left(k \lambda_{e}\right)^{2}}+\frac{3 T_{e}}{T_{i}}\right]
$$

The light scattered from these fluctuations will be Doppler shifted by the acoustic phase velocity $\left(\mathrm{c}_{s}\right)$. Two peaks will be observed in the scattered spectrum; light shifted to the blue corresponds to light scattered from an acoustic wave with a velocity component towards the detector, and light shifted to the red corresponding to light scattered from an acoustic wave with a velocity component away from the detector [Fig. 4.2]. The separation between the two peaks in the spectrum is proportional to the sound speed of the ion-acoustic wave (see Sec. 2.2),

$$
\Delta \lambda_{i a w}=2 \frac{\lambda_{t s}^{2}}{2 \pi c}\left(c_{s} k_{a}\right)
$$

where $\lambda_{t s}$ is the wavelength of the probe laser and $\mathrm{k}_{a}$ is the wave number of the ion-acoustic wave. If there is an ion flow relative to the laboratory frame, then the scattered light will have an additional shift given by,

$$
\Delta \lambda_{\text {flow }}=\frac{\lambda_{t s}^{2}}{2 \pi c}\left(v_{f} k_{a}\right)
$$

\subsection{Thomson scattering parameter}

The scattering parameter $(\alpha)$ allows one to determine whether the measured light is characterized by single particle behavior (short wavelength fluctuations) or light scattered 


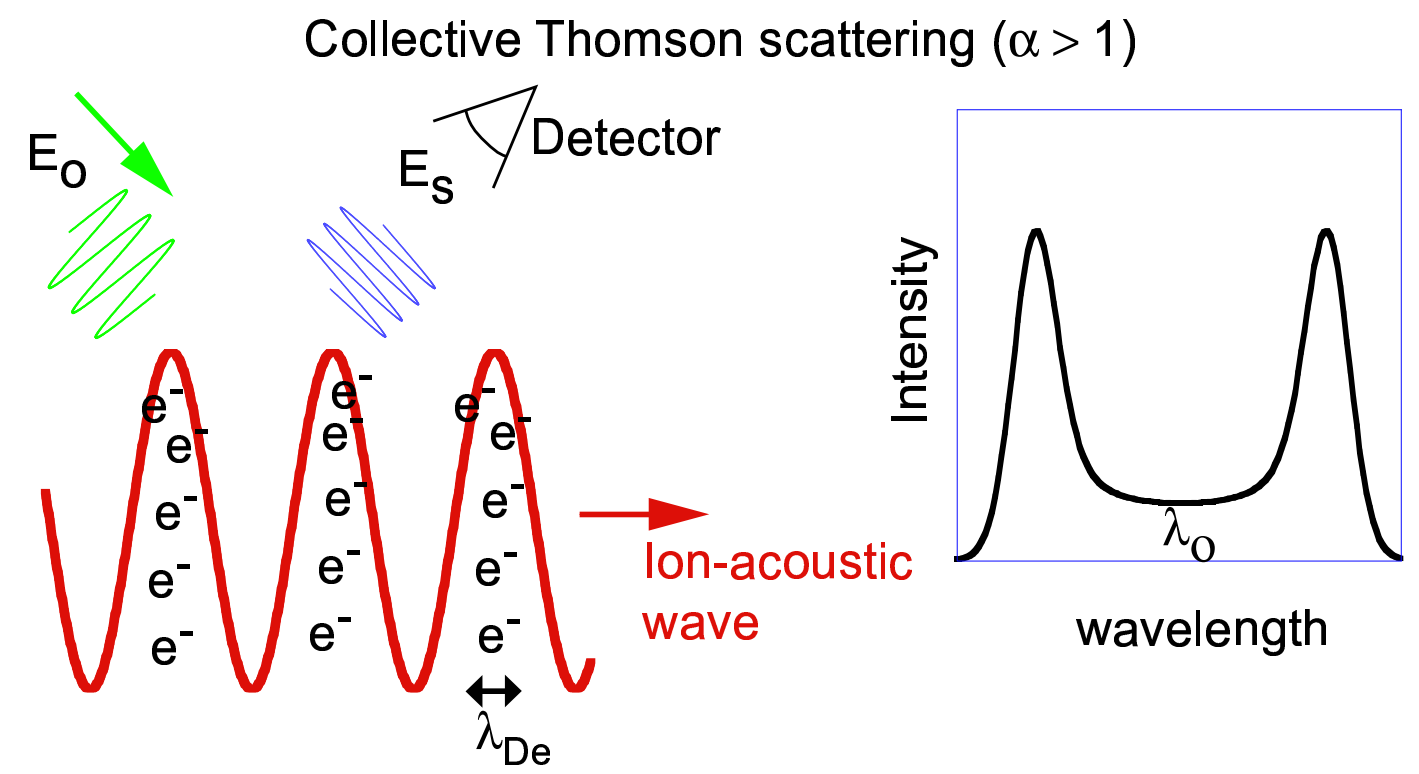

Figure 4.2: (color). Light scattered from low frequency fluctuations reveals two peaks corresponding to co- and counter-propagating ion-acoustic waves.

from collective motion (long wavelength fluctuations). The scattering parameter is the ratio of the fluctuation wavelength $(\lambda)$ to the Debye length $\left(\lambda_{D e}\right)$,

$$
\alpha=\frac{1}{k \lambda_{D e}} \propto \frac{\lambda}{\lambda_{D e}}
$$

When the scattering parameter is large $(\alpha>>1)$, then the wavelength of the probed fluctuation carries over many Debye lengths and wave-like behaviors are probed (collective scattering). When the scattering parameter is small $(\alpha<<1)$, the fluctuation wavelength is shorter than the Debye length and individual electron motion is probed (non-collective scattering). The probed wavelength can be determined by selecting the wavelength of the incident probe laser light $\left(k_{t s}=\frac{2 \pi}{\lambda_{t s}}\right)$, the wavelength of the observed light $\left(k_{s}=\frac{2 \pi}{\lambda_{s}}\right)$, and the direction of observation relative to the incident light $(\theta)$ [Fig. 4.3].

When the wavelength shift between the scattered light and the incident light is 


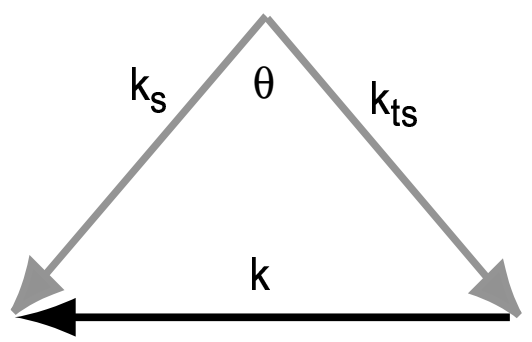

Figure 4.3: The wave vector of the fluctuations are determined by momentum $\left(\mathbf{k}_{\mathbf{t s}}=\mathbf{k}_{\mathbf{s}}+\mathbf{k}\right)$ and energy $\left(\omega_{t s}=\omega_{s}+\omega\right)$ conservation.

small $\left(k_{s} \approx k_{t s}\right)$ then,

$$
k=2 k_{t s} \sin \frac{\theta}{2}
$$

This condition holds for scattering from low-frequency ion-acoustic fluctuations, but not for scattering from higher-frequency electron plasma waves.

\subsection{Thomson scattering theory}

The time-averaged Thomson scattered power $\left(P_{s}\right)$ per unit solid angle $(\Omega)$ per unit frequency $(\omega / 2 \pi)$ is given by [22],

$$
\frac{d P_{s}}{d t d \Omega d \omega / 2 \pi}=\frac{P_{i}}{r_{o}^{2} A}\left(1-\sin ^{2} \theta \cos ^{2}\left|\phi-\phi_{o}\right|\right) N_{e} S(\mathbf{k}, \omega)
$$

where $r_{o}=\frac{e^{2}}{m c^{2}}$ is the classical electron radius, $P_{i}$ is the incident power, $\mathrm{A}$ is the cross sectional area of the incident beam, $N_{e}$ is the number of electrons in the scattering volume, V. The wave vector and frequency of the probed fluctuation $(\mathbf{k}, \omega)$ is the difference in the wave vector and frequency between the probe $\left(\mathbf{k}_{\mathbf{t s}}, \omega_{\mathbf{t s}}\right)$ and the scattered $\left(\mathbf{k}_{\mathbf{s}}, \omega_{\mathbf{s}}\right)$ 
electromagnetic waves [Fig. 4.3],

$$
\begin{aligned}
& \mathbf{k}=\mathbf{k}_{\mathbf{t s}} \pm \mathbf{k}_{\mathbf{s}} \\
& \omega=\omega_{t s}-\omega_{s} .
\end{aligned}
$$

$\theta$ is the scattering angle, and $\phi-\phi_{t s}$ is the angle between the incident electric field vector, and $\mathbf{k}_{\mathbf{s}}$ in a plane perpendicular to $\mathbf{k}_{\mathbf{t s}}$.

The single-ion species dynamic form factor for a collisionless plasma,

$$
S(\mathbf{k}, \omega)=\frac{2 \pi}{\mathbf{k}}\left|\mathbf{1}-\frac{\chi_{\mathbf{e}}}{\varepsilon}\right|^{2} \mathbf{f}_{\mathbf{e}}\left(\frac{\omega}{\mathbf{k}}\right)+\frac{2 \pi \mathbf{Z}}{\mathbf{k}}\left|\frac{\chi_{\mathbf{i}}}{\varepsilon}\right| \mathbf{f}_{\mathbf{i}}\left(\frac{\omega}{\mathbf{k}}\right)
$$

was determined in 1960 by Fejer [17] using a linearized Vlasov equation to express the particle motion within a plasma. In the above equation, $f\left(\frac{\omega}{\mathbf{k}}\right)$ are normalized one-dimensional distribution functions (Eq. 2.1) in the direction of $\mathbf{k}$ evaluated at the phase velocity $v_{\phi}=\frac{\omega}{\mathbf{k}}$. $\mathrm{Z}$ is the average electron charge state. $\varepsilon(\mathbf{k}, \omega)=1+\chi_{e}+\chi_{i}$ is the plasma dielectric function and $\chi_{e}, \chi_{i}$ are the electron, ion susceptibilities discussed in Sec. 2.1.2. It is evident from Eq. 4.9 that peaks will appear in the scattered spectrum whenever $\operatorname{Re}(\varepsilon)=0$, the natural modes of the plasma. An ion-acoustic wave corresponds to a weakly damped lowfrequency natural mode, therefore, one expects for a single-ion-species plasma, two peaks in the scattering spectrum corresponding to wave-vectors $\pm \mathrm{k}$. The wavelength separation of the two peaks is twice the phase velocity of an ion-acoustic wave which is given by the linear dispersion relation (Sec. 2.2.1),

$$
v_{\phi}^{2}=\frac{k_{b} T_{e}}{M_{i}}\left[\frac{Z}{1+\left(k \lambda_{e}\right)^{2}}+\frac{3 T_{e}}{T_{i}}\right]
$$

For a high-Z plasma or for $\frac{T_{i}}{T_{e}}<<1$, the second term in Eq. 4.10 can be neglected. In this case the frequency separation of the ion-acoustic peaks is proportional to the electron tem- 
perature. The wavelength separation between the two peaks is $\Delta \lambda=2 \omega_{a} \lambda_{o}^{2} / 2 \pi c$. Therefore, we find from Eqs. 4.5 and 4.10,

$$
\Delta \lambda=\frac{4 \lambda_{t s}}{c} \sin \left(\frac{\theta}{2}\right) \sqrt{\frac{Z k_{b} T_{e}}{M_{i}\left(1+k^{2} \lambda_{e}^{2}\right)}} .
$$

It is clear from Eq. 4.11, that the separation of the two acoustic peaks in the Thomson spectra allows the measure of the electron temperature when the average charge state $(\mathrm{Z})$ is known.

\subsection{Multi-ion species Thomson scattering spectra}

Adding a second ion species to a plasma introduces another set of acoustic modes, therefore, two additional peaks are expected in the Thomson scattering spectra. In 1970 Evans [23] adapted the theoretical form factor to include multi-ion-species plasmas,

$$
S(\mathbf{k}, \omega)=\frac{\mathbf{2} \pi}{\mathbf{k}}\left|\mathbf{1}-\frac{\chi_{\mathbf{e}}}{\varepsilon}\right|^{\mathbf{2}} \mathbf{f}_{\mathbf{e}}\left(\frac{\omega}{\mathbf{k}}\right)+\frac{\mathbf{2 \pi}}{\mathbf{k}} \sum_{\alpha} \mathbf{Z}_{\alpha}\left|\frac{\chi_{\alpha}}{\varepsilon}\right| \mathbf{f}_{\alpha}\left(\frac{\omega}{\mathbf{k}}\right)
$$

Figure 4.4 is a plot of the calculated scattering power spectrum, Eq. 4.6, using the multiion form factor (Eq. 4.12) for a Be plasma with the addition of $10 \%$ Au. By varying the electron temperature while holding all other parameters fixed $\left(T_{i}=250 \mathrm{eV}, Z=4, n_{e}=\right.$ $1.5 \times 10^{15} \mathrm{~cm}^{-3}$ ), three spectra are plotted showing the sensitivity of the form factor to the electron temperature (Fig. 4.4). The peaks with the greatest separation correspond to a fast ion-acoustic mode, Eq. $2.18\left(\mathrm{v}_{\phi} / c=4.2 \times 10^{-4}\right)$. The smaller, less shifted peaks, correspond to a slow ion-acoustic mode, Eq. $2.19\left(\mathrm{v}_{\phi} / c=9 \times 10^{-5}\right)$. For our conditions, there is a weakly damped fast mode (the normalized Landau damping from Sec. 2.3 is $\nu_{L} / \omega=0.05$ ) and a larger damped slow mode $\left(\nu_{L} / \omega=0.3\right)$. 

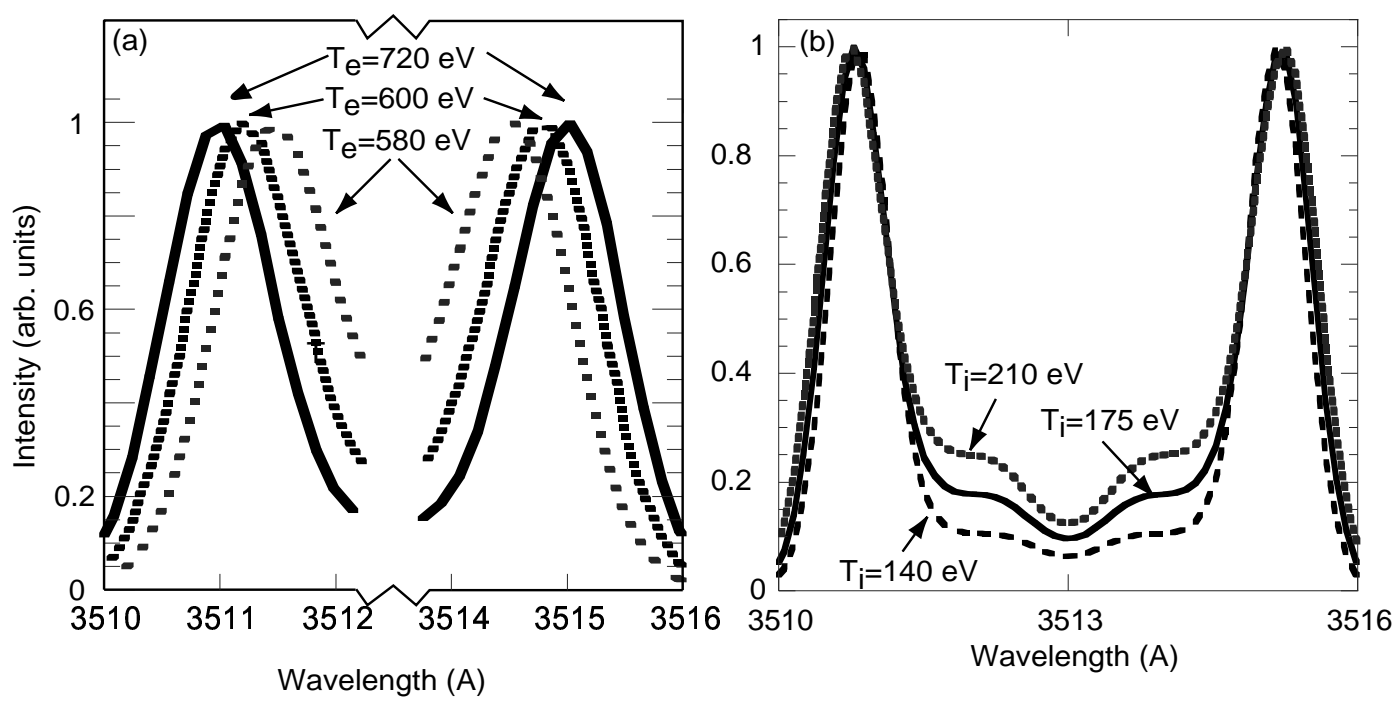

Figure 4.4: (a) The theoretical form factor is plotted for $20 \%$ variations in electron temperature, (b) and ion temperature while all other parameters are held fixed.

As in the single species case, the separation between the peaks corresponding to a particular mode is proportional to the phase velocity of the mode (if the modes are well separated) therefore, if the charge state of one of the species is known, then the electron temperature, and the charge state of the second species can be measured (See Eqs. 2.18 and 2.19).

Furthermore, the two-ion-species plasmas allow us to measure the ion temperature in a unique way; the relative amplitude of the peaks in the Thomson-scattering spectra belonging to the slow and fast ion-acoustic waves provide an accurate measure of the ion temperature. As a result of the sensitivity of Landau damping to ion temperature (see Sec. 2.3), the damping of the fast mode increases while the damping of the slow mode decreases when the ion temperature is increased. Figure $4.4 \mathrm{~b}$ illustrates the respective increase or decrease in the amplitude of the peaks in the Thomson scattering spectra when 


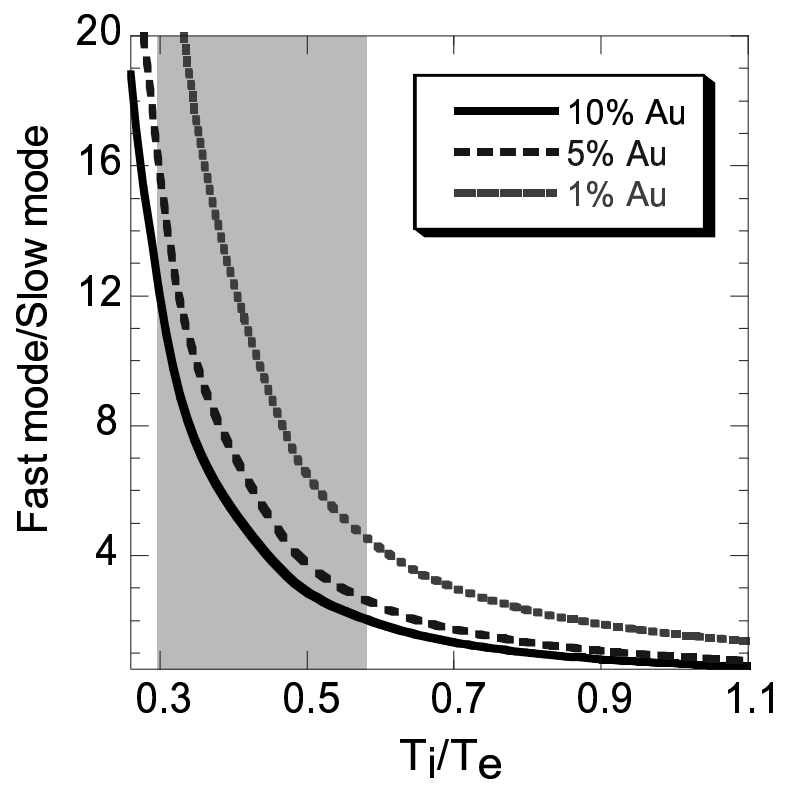

Figure 4.5: The ratio between the amplitude of the light scattered from the fast mode to the amplitude of the light scattered from the fast mode is plotted as a function of ion temperature for a fixed electron temperature, $\mathrm{T}_{e}=600 \mathrm{eV}$. The shaded area represents the experimental region.

the ion temperature varies. Figure 4.5 indicates the sensitivity of the Thomson-scattering spectra to the ion temperature; the ratio between the amplitudes of the slow and fast mode is plotted as a function of ion temperature while all other parameters are held constant. In practice, the average charge state, the velocity flow, the electron and ion temperatures are determined through a four parameter fit of the form factor, Eq. 4.12.

\subsection{Scattering from stimulated Brillouin excited acoustic waves}

When a high power laser is incident on a plasma, the resulting ponderomotive force will excite acoustic waves. When the wave number of the excited acoustic fluctuation is twice the wave number of the interaction beam $\left(k=2 k_{o}\right)$, a process called stimulated Brillouin 
scattering (SBS) will amplify the acoustic wave (see Chapt. 3). Using Eq. 4.5 it is evident that if the angle between the Thomson-scattering probe laser and the Thomson-scattering collection optics is $\theta=84^{\circ}$ (assuming $\lambda_{t s}=351 \mathrm{~nm}$ for the Thomson probe laser and $\lambda_{o}=$ $527 \mathrm{~nm}$ for the interaction beam), then one can observe Thomson-scattered light scattered from the SBS excited acoustic waves $\left(\mathrm{k}=2 \mathrm{k}_{o}\right)$. The intensity of the Thomson-scattered light is proportional to the square of the amplitude of the ion-acoustic wave $\left(\frac{\delta n}{n_{e}}\right)$ that is being probed, therefore, Thomson scattering is a unique tool to investigate this process. In the presence of an interaction beam only the ion-acoustic wave co-propagating in the direction of the interaction beam will be excited; therefore, only the red shifted Thomson-scattered light will be amplified. Figure 4.6 shows a representative scattering spectra in which ionacoustic waves have been excited above the thermal level by the interaction beam. The square of the amplitude of the ion-acoustic wave is proportional to the scattered intensity, therefore, we define the normalized ion wave amplitude as,

$$
\frac{d n}{n_{e}} \propto \sqrt{\frac{I_{\text {excited }}}{I_{\text {thermal }}}}
$$




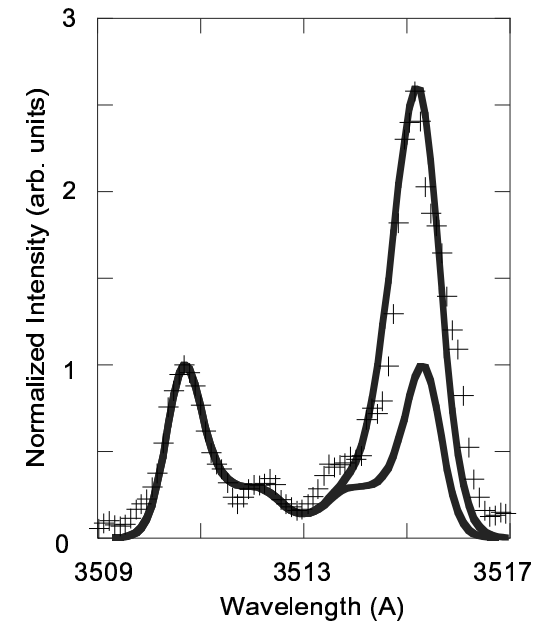

Figure 4.6: A Thomson scattering spectrum is shown for a shot in which the red-shifted peak has been excited by SBS. The blue-shifted peaks are fit with the standard multi-ion-species form factor allowing the measure to the ion temperature, $\mathrm{T}_{i}=475 \mathrm{eV}$. 


\section{Part II}

\section{Experiment}




\section{Chapter 5}

\section{Experimental Setup}

Experiments were carried out in two series of tests at the Trident Laser Facility. The first was a three week campaign that began in March of 2001. The second was a four week campaign that began in April of 2002. A total of 160 shots were used in these experiments. In the first campaign, we employed two main diagnostics: the stimulated Brillouin excited ion-acoustic wave was measured using one Thomson scattering configuration, and a diode was used to measure the total backscattered energy. The second campaign added to these measurements; a second Thomson scattering diagnostic was used to measure thermal ion-acoustic waves in the same volume of plasma as the first, while two spectrometers were used to observe the spectra of the stimulated Raman and stimulated Brillouin backscattered light. 


\subsection{Trident Laser Facility}

The Trident Laser Facility is located at the Los Alamos National Laboratory and is used as a multipurpose laboratory for developing instrumentation and conducting experiments requiring short (nanosecond) low energy (sub-kilojoule) laser-light pulses [24]. The Trident facility includes a Nd:glass laser driver that is split into three laser beams. The laser facility was upgraded to its current configuration in 1992 when LANL acquired the Chroma laser [25].

The Trident Facility employs a Nd:YLF master oscillator and a chain of Nd:phosphate glass rod and disk amplifiers that produce pulse lengths from 100 ps to 2 ns. The laser can generate $60 \mathrm{~J}$ in $100 \mathrm{ps}$ and $250 \mathrm{~J}$ in $2 \mathrm{~ns}$ in each of two frequency-doubled $(\lambda=527 \mathrm{~nm}$ frequency doubled from $\lambda=1054 \mathrm{~nm}$ ) main drive beams, and it can generate $1 \mathrm{~J}$ in $200 \mathrm{ps}$ of frequency tripled $(\lambda=351 \mathrm{~nm})$ light in a third beam line.

\subsection{Overview of experimental configuration}

The experiments used a configuration in which three laser beams converge at a target within a vacuum chamber in the target room at the Trident Laser Facility. The plasma was produced by a "heater beam" of $2 \omega(\lambda=527 \mathrm{~nm})$ laser light focused to an intensity of $10^{14} \mathrm{~W} \mathrm{~cm}^{-2}$. A separate high energy $2 \omega$ "interaction beam", with a $1.2 \mathrm{~ns}-$ long square pulse, was aligned parallel to the target and focused to a maximum laser intensity of $7 \times 10^{15} \mathrm{~W} \mathrm{~cm}^{-2}[26]$. The interaction beam was used to drive stimulated Brillouin scattering which excites ion-acoustic waves in the plasma. The wave number of the excited ion wave was matched using a third $3 \omega\left(\lambda_{t s}=351 \mathrm{~nm}\right) 200 \mathrm{ps}$ long Thomson-scattering 


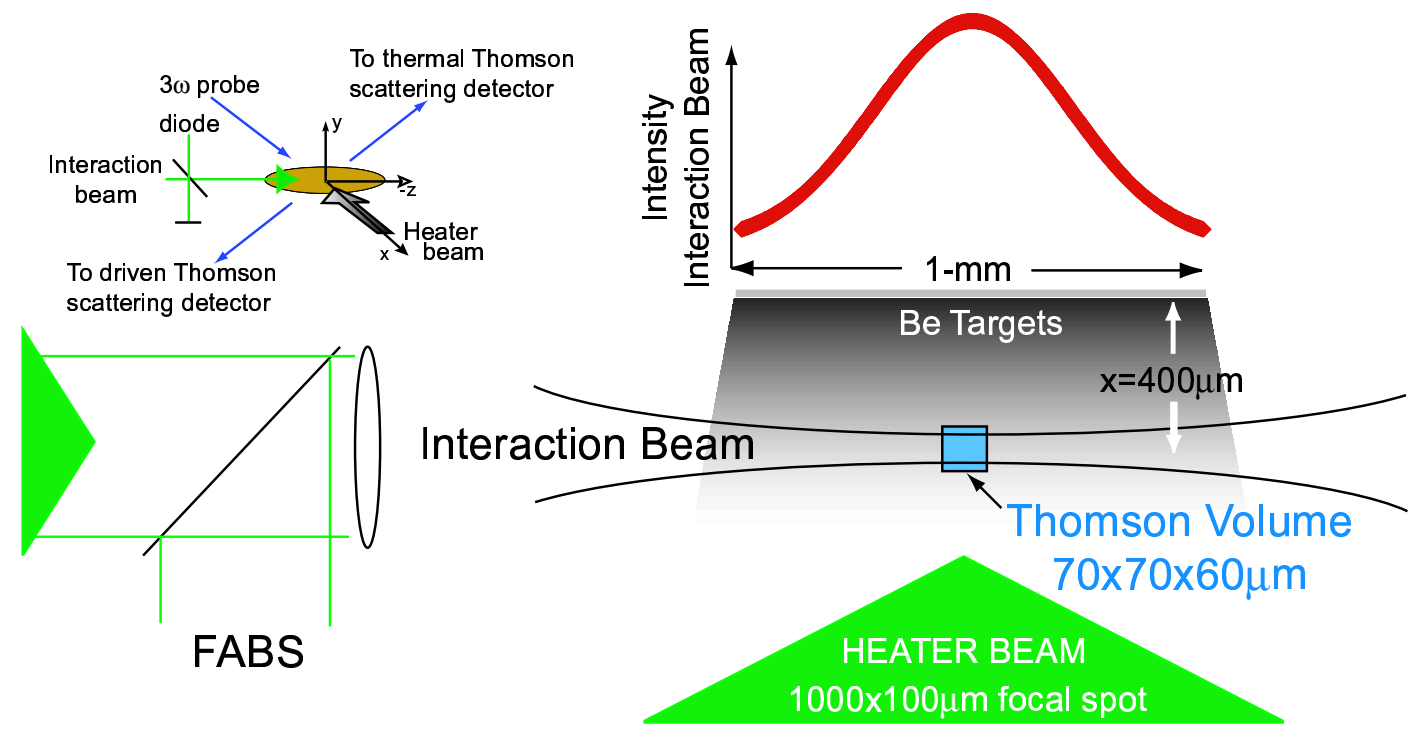

Figure 5.1: Schematic of the experimental setup.

\begin{tabular}{|l|c|c|c|c|c|c|}
\hline & Beam & Wavelength & Energy & Pulse Length & Spot Size & Max. Intensity \\
\hline & & $(\mathrm{nm})$ & $(\mathrm{J})$ & $(\mathrm{ns})$ & $(\mu \mathrm{m})$ & $\left(\mathrm{W}-\mathrm{cm}^{-2}\right)$ \\
\hline Interaction Beam & $\mathrm{A}$ & 527 & $0-230$ & 1.2 & 60 & $7 \times 10^{15}$ \\
\hline Heater Beam & $\mathrm{B}$ & 527 & 180 & 1.2 & $1000 \mathrm{x} 100$ & $2 \times 10^{14}$ \\
\hline Probe Beam & $\mathrm{C}$ & 351 & 1 & 0.200 & 60 & $2 \times 10^{14}$ \\
\hline
\end{tabular}

Table 5.1: Laser Beam Parameters

"probe beam". Table 5.1 lists the laser beam parameters used in the experiments.

\subsubsection{Beam transport}

The heater beam (Beam B) enters through beam tubes in the north-west corner of the target room [Fig. 5.2]. The first two mirrors are used to adjust the path length of the heater beam and, thereby, the relative timing between the heater beam and interaction beam while the third mirror directs the beam to the target chamber center. The heater beam was directed normal to the target surface and focused to a plane at target chamber 
center [Fig. 5.1] by a $120 \mathrm{~cm}$ focal length $f / 6$ lens and a strip line random phase plate (RPP). This produced a line focus with a $1000 \mu \mathrm{m} \times 100 \mu \mathrm{m}$ elliptical area with an intensity of $10^{14}$ $\mathrm{W} \mathrm{cm}^{-2}[27]$.

The interaction beam (Beam A) was transported via three mirrors before passing through the backscatter wedge (4\% reflection) [Fig. 5.2]. The probe beam (Beam C) entered the room between and above Beams A and B. The probe beam is frequency tripled in the target room [Fig. 5.3]. The $1 \omega$ beam is transported through the upper table down a periscope where it pass through a KDP trippler and out towards the target chamber. The beam is then reflected off two mirrors before entering target chamber [Fig. 5.4]. The linearly polarized light from the probe beam was set in the plane normal to the target surface.

The interaction beam and probe beams (Beams A and C) both run parallel to the target surface. They are focused to a volume at target chamber center a distance $\mathrm{x}$ from the target surface using a $120 \mathrm{~cm}$ focal length $f / 6$ and a $700 \mathrm{~cm}$ focal length $f / 10$ lens respectively (see 5.1). In both cases a best focus diameter of about $60 \mu \mathrm{m}$ was achieved. The spot size of the interaction beam was previously determined by Ref. [26] by imaging the interaction beam onto an equivalent focal plane at a CCD camera as shown in Fig. 5.5.

\subsubsection{Pulse shaping}

The oscillator output pulse (200 ps) is temporally shaped using a pulse stacker [28]. The pulse stacker consists of 13 elements that can be selected to set the length of the pulse and its timing relative to the probe beam. Each element allows one pass of the input pulse and therefore adds 200 ps to the output pulse. For example, if elements 1 through 6 are selected, then the output pulse will be 1.2 ns. Furthermore, the attenuation of each element 


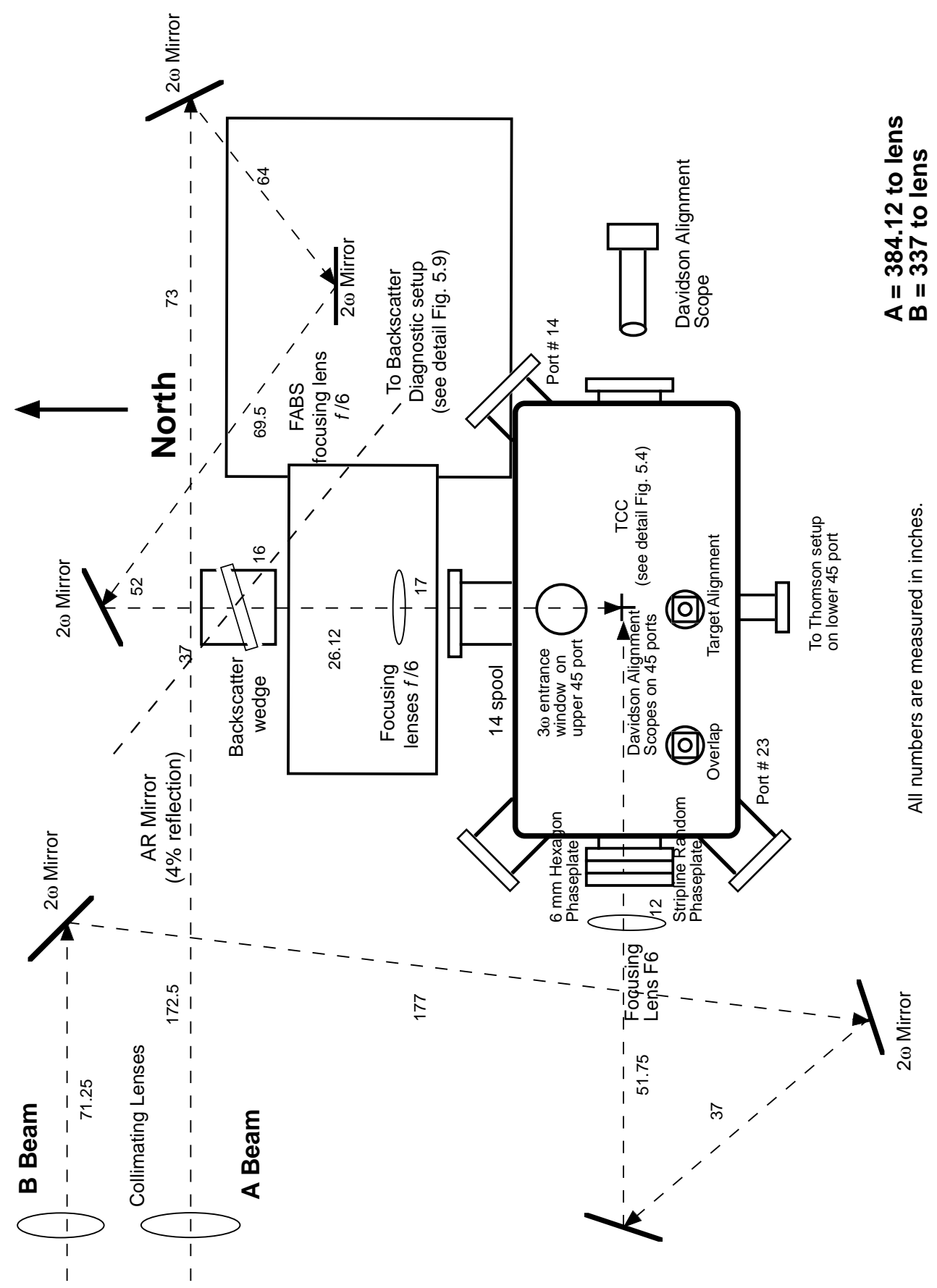

Figure 5.2: Beam Transport is shown for both the interaction and heater beams, A and B respectively. 


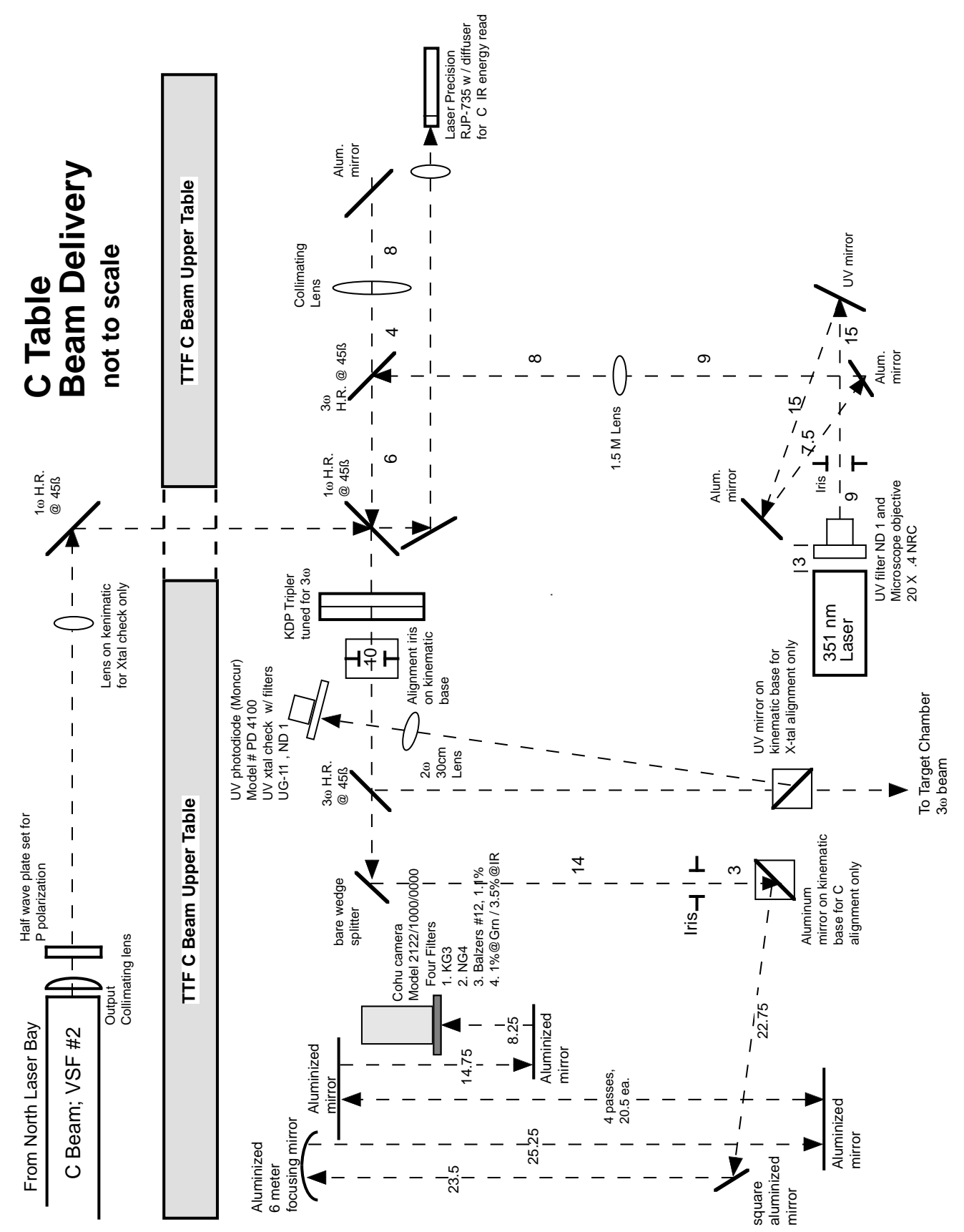

Figure 5.3: The beam delivery is shown for the probe beam (Beam C). 


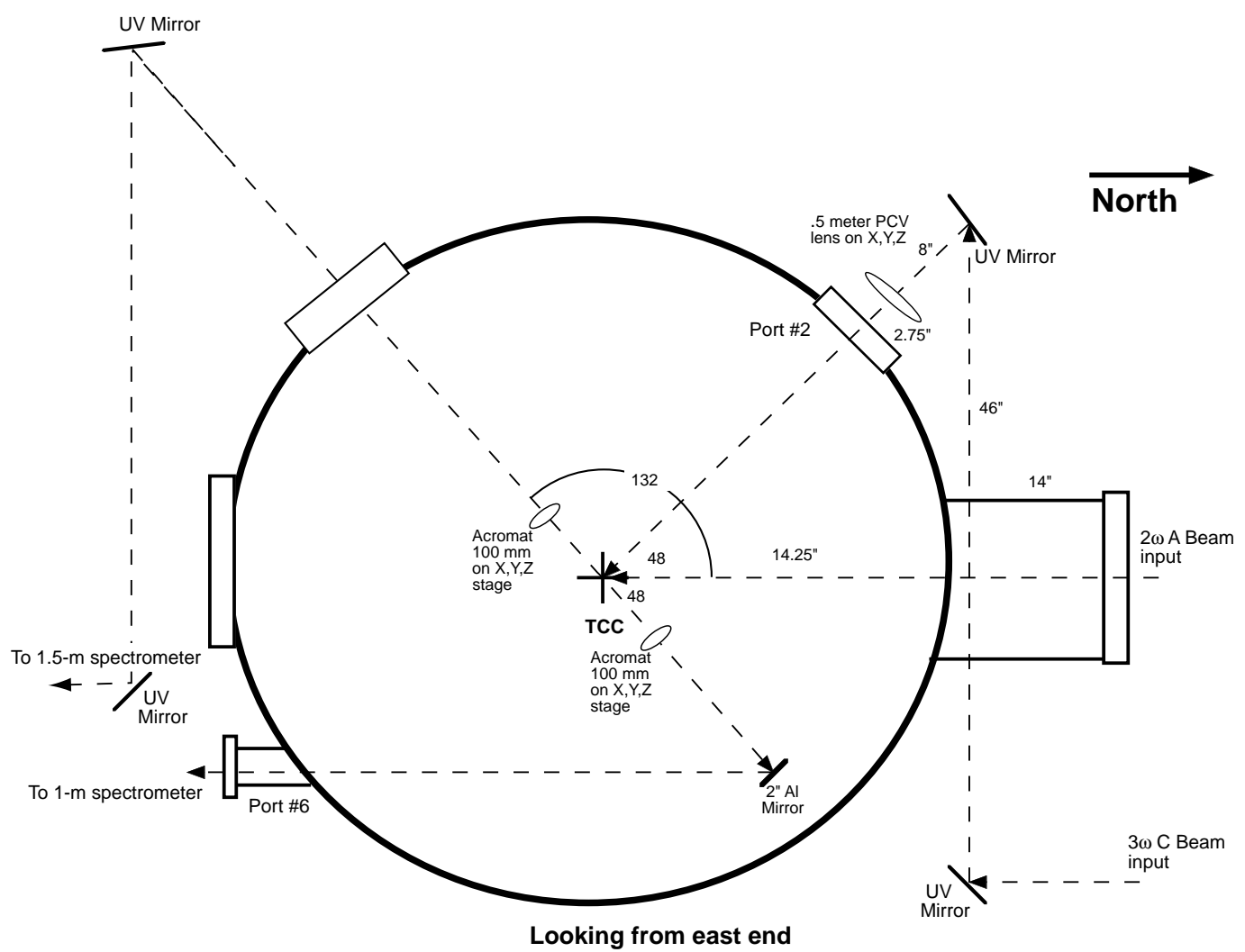

Target chamber setup

Figure 5.4: The east view of the target chamber is shown providing an overview of the probe beam transport to target chamber center. The collection optics for both Thomsonscattering diagnostics are shown. 


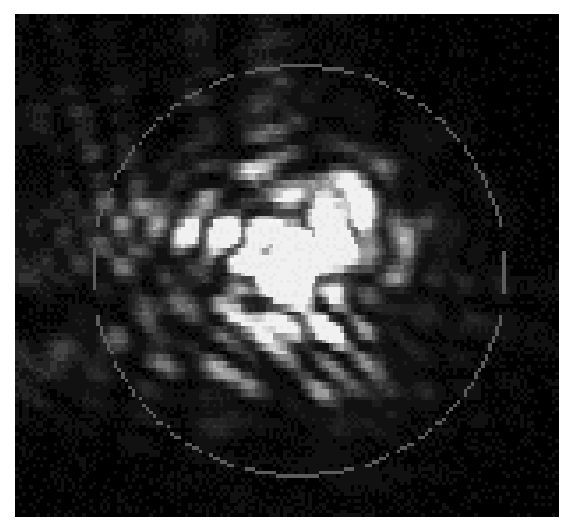

Figure 5.5: An image of the focal spot of beam A taken on shot 5279 with a $75 \mu \mathrm{m}$ circle superimposed. In this image, low level, large area wings that account for about $1 / 3$ of the total energy are visible.

can be selected to shape the pulse.

The stacked pulses are then amplified and split into the A and B drive beams (Fig. 5.6). These beams are further amplified to a maximum energy of $250 \mathrm{~J}$ in $2 \mathrm{~ns}$. The drive beams are expanded throughout the amplification stages to a final diameter of $20 \mathrm{~cm}$. The timing between the drive beam pulses at the target is determined by their relative path lengths.

The probe beam ( $\mathrm{C}$ beam) can be selected in one of two configurations: with the same pulse shape as the drive beams, or the Gaussian pulse shape from the oscillator. In the former, the relative probe beam timing is determined by its path length to the target. In the latter configuration, the 200 ps Gaussian pulse is split to the probe beam amplifiers before entering the pulse stacker. In this case the system is more flexible and timing can be changed without realignment to the target chamber center. The timing between the drive beams and the probe beam is simply adjusted by: a trombone delay system just after the oscillator, and/or the elements selected in the pulse stacker (Fig. 5.6). 


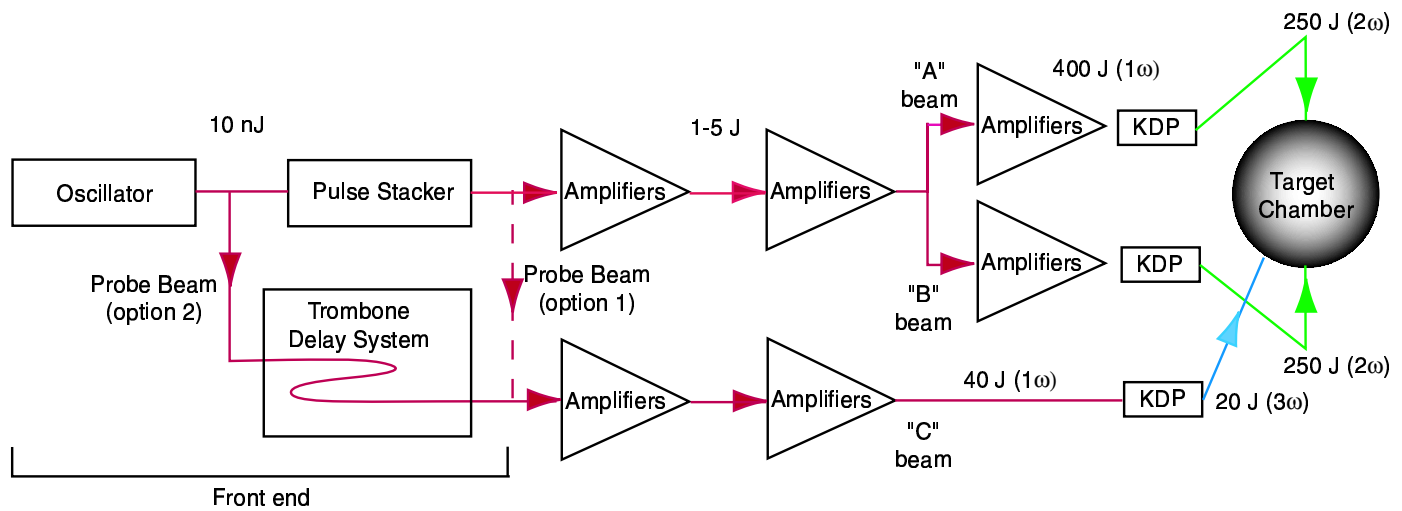

Figure 5.6: (color). Trident's driver laser uses a conventional master oscillator power amplifier architecture. Drive (A \& B beams) and probe (C beam) pulses can be amplified sequentially through the front end before being routed to separate disk amplifier chains. If a short pulse is desired for beam C, the 200 ps pulse from the oscillator can be picked off before the pulse stacker.

For example, if the drive beams are 1.2 ns long ( 6 elements would need to be used for shaping), the probe beam timing relative to the drive beams can be varied by adding non-alternating elements. So if elements 1 through 6 are used, then selecting elements 4 through 9 for the drive beams would change the timing by 800 ps. Therefore, the timing can easily be adjusted in 200 ps steps over a 1.2 ns range by selecting the elements used to transmit the drive beams. Our experiments use the Gaussian configuration.

\subsubsection{Beam timing}

The interaction beam and heater beam arrive at the target room at approximately the same time. The path length the interaction beam travels in the target room is slightly longer than the heater beam. The time delay was measured using a fast Hamamatsu photo diode placed at target chamber center. One 200 ps element was selected from the pulse train; Figure 5.7 a shows the two pulses arriving at TCC $1.0 \mathrm{~ns}$ apart. The first pulse is 

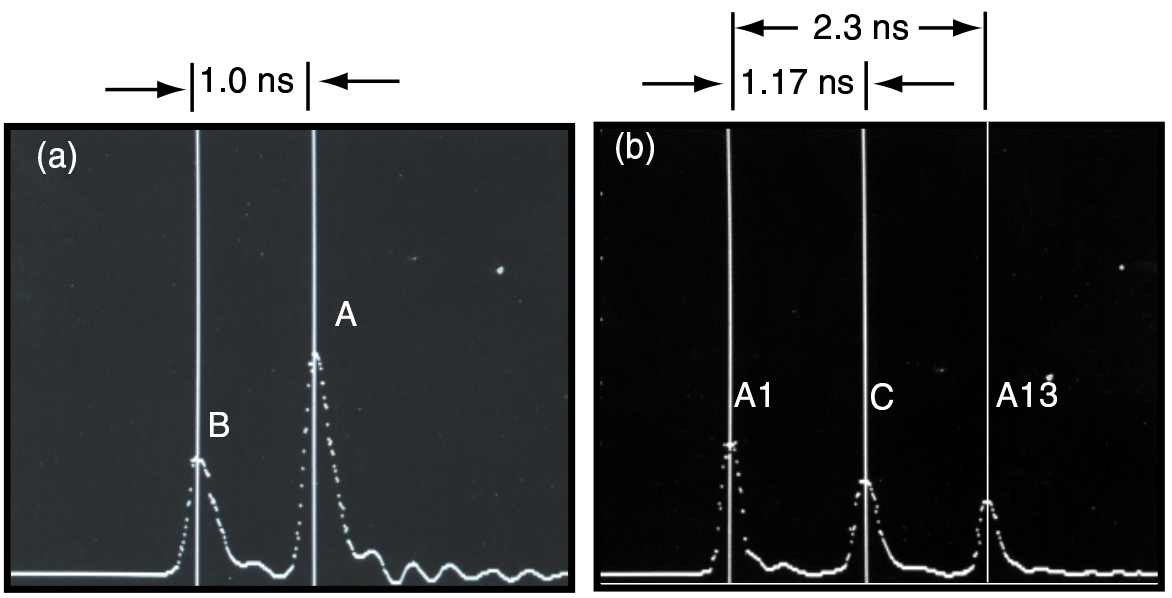

Figure 5.7: (a) One element was selected; the relative timing between beams A and B are measured at TCC. (b) Elements A1, A13 and beam $\mathrm{C}$ were selected; the relative timing between elements A1 and A13 was measured to be $2.34 \mathrm{~ns}$; this indicates that there is 180 ps between elements. The timing between element $\mathrm{A} 1$ and $\mathrm{C}$ was 1.17. The heater beam path (beam B) was blocked. These results are from campaign 2 .

\begin{tabular}{|c|c|c|c|}
\hline & A & B & C \\
\hline Campaign 1 & 830 & 0 & 1400 \\
\hline Campaign 2 & 1000 & 0 & 1410 \\
\hline
\end{tabular}

Table 5.2: The relative beam timings are shown for two Trident campaigns. In campaign 1, elements 6-13 where used. In Campaign 2, elements 5-11 where selected. All numbers are in picoseconds.

from the heater beam, while the second is from the interaction beam.

The time delay measured at the target chamber center between the first element of beam $\mathrm{A}$ and beam $\mathrm{C}$ is shown in Figure $5.7 \mathrm{~b}$ to be $1.17 \mathrm{~ns}$. The beam timings for our two campaigns are shown in Table 5.2. 


\begin{tabular}{|c|c|c|c|c|c|c|}
\hline & Spectrometer Length & Order & Grating & Slit Width & Dispersion & Instrument Function \\
\hline & (meters) & & (grooves / mm) & (microns) & A / pxl & A \\
\hline dts1 & 1.0 & 1 & 3600 & 200 & 0.055 & 0.47 \\
\hline $\mathrm{dts} 2$ & 0.5 & 2 & 1200 & 200 & 0.10 & 0.25 \\
\hline $\mathrm{tts}$ & 1.5 & 1 & 2400 & 200 & 0.05 & 0.02 \\
\hline $\mathrm{sbs}$ & 1.0 & 1 & 1200 & 200 & 0.05 & 0.45 \\
\hline $\mathrm{srs}$ & 0.25 & 1 & 150 & 300 & 5.3 & \\
\hline
\end{tabular}

Table 5.3: The spectrometer characteristics are tabulated above.

\subsection{Instrumentation}

\subsubsection{Spectrometer characterization}

Five separate spectrometers were used. Hamamatsu streak cameras were coupled to the output of the spectrometers by placing the input slit of the streak camera at the image plane of the spectrometer. The instrument calibration was determined using a $\mathrm{Ne}$ lamp placed at the slit of the spectrometer [Fig. 5.8]. The results of these measurements are compiled in Table 5.3.

\subsection{Full aperture backscatter station configuration}

\subsubsection{Backscatter beam transport}

The Full Aperture Backscatter Station (FABS) collects the interaction beam light backscattered by the plasma. This backscattered light was collimated by the interaction beam focusing lens as it propagated back up the laser chain [Fig. 5.9]. Four percent of the backscattered light was diverted by the backscatter wedge. The reflection from the front surface of the wedge was then focused by the FABS f/6 $(120 \mathrm{~cm}$ focal length) lens on to 

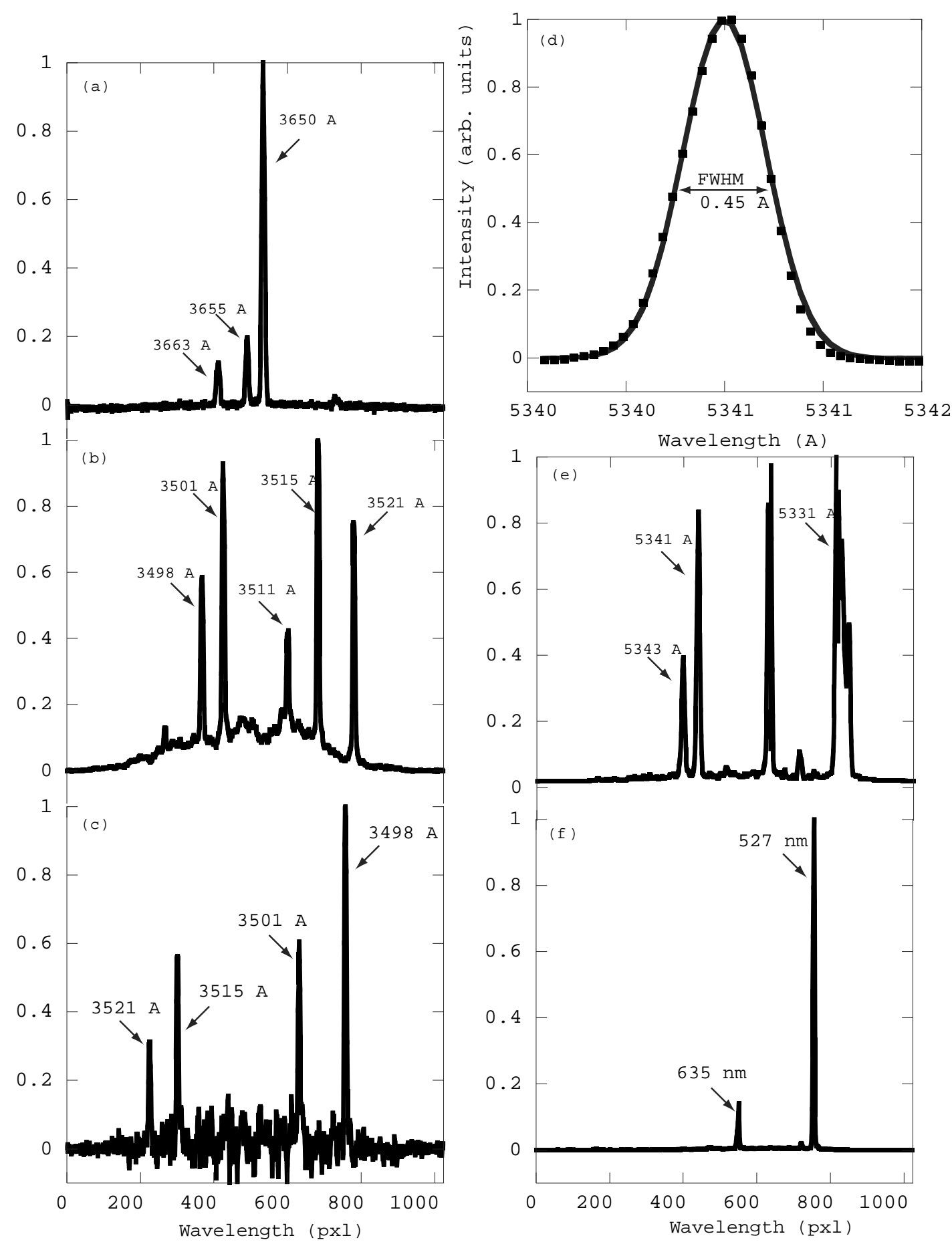

Figure 5.8: A Ne spectra was measured with each spectrometer (except the 1/4 meter) to calculate the instrument function and the dispersion. (a) 1/2-meter dts (b) 1-meter dts (c) 1.5-meter tts (d) instrument function for one Ne-line from the 1-meter sbs spectrometer (e) 1 -meter sbs (f) The 1/4-meter spectrometer was calabrated using two laser lines at $632 \mathrm{~nm}$ and $527 \mathrm{~nm}$. 
a large area diode. Prior to the diode there was a bare wedge. The reflections from the front and rear surfaces of the wedge were focused onto fibers that were coupled to respective 1-meter and 1/4-meter spectrometers.

\subsubsection{Backscatter energy measurement}

The backscattered energy was measured using a fast (200 ps rise time) Hamamatsu large area photodiode. The backscattered light was focused through a band pass filter $(527 \pm 10 \mathrm{~nm})$ to eliminate stray light. To calibrate the backscattered energy, $4 \%$ of the interaction beam was split by the back surface of the backscatter wedge [Fig. 5.9]. A mirror with an anti-reflection (AR) coating on the back surface was used to reflect $4 \%$ of the split interaction beam into the FABS focusing lens. The path length between the reference pulse and the backscattered light was adjusted such that the calibration pulse arrived at the diode approximately 15 ns prior to the backscattered signal [Fig. 5.10]. The data was reduced by numerically integrating each pulse; the SBS reflectivity is the ratio of the integrated signals adjusted for the energy loss (96\%) at the AR mirror,

$$
R=4 \%\left(\frac{I_{s b s}}{I_{\text {interaction beam }}}\right)
$$

\subsubsection{Backscatter spectral measurements}

The spectrum of the backscattered light was measured by coupling the light into two $400 \mu \mathrm{m}$ fiber optic cables. Ten meter fibers were placed at the focus of the FABS focusing lens. The light was spatially smoothed by placing $3^{\circ}$ diffusers $5 \mathrm{~cm}$ from the fiber optic entrances. The diffuser expanded the spot on the fiber to approximately $2 \mathrm{~mm}$ 


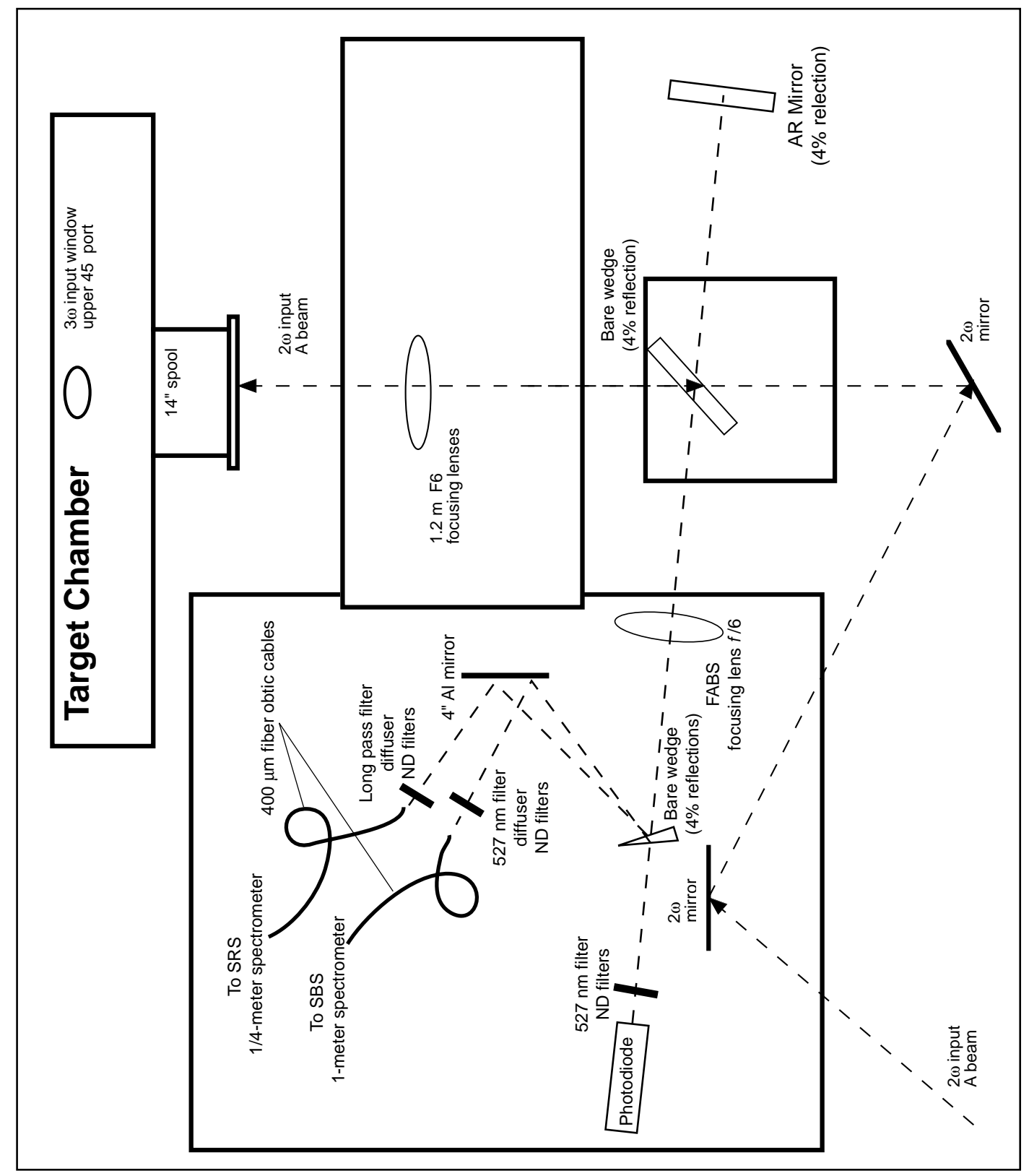

Figure 5.9: FABS block diagram. 


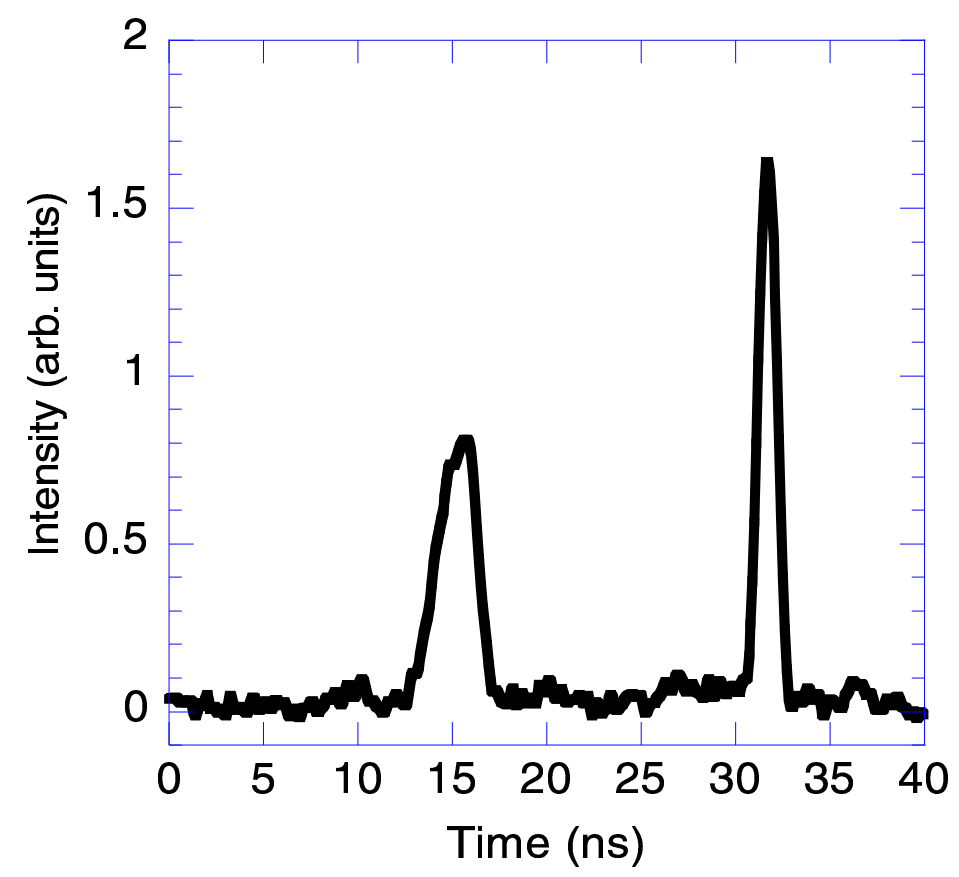

Figure 5.10: A representative diode trace is shown for a Au shot with an interaction beam energy of $190 \mathrm{~J}$ (shot 050701069). The integrated signals are roughly equal corresponding to a $4 \%$ reflectivity. 
diameter making alignment less critical. The fiber optic cables where mounted at the slits of a 1-meter and 1/4-meter spectrometer. The spectral resolution of the 1-meter and 1/4meter spectrometers was $0.05 \mathrm{~nm}$ and $0.5 \mathrm{~nm}$ respectively (See Section 5.3.1). The 1-meter spectrometer was used to observe the backward stimulated Brillouin scattered light while the 1/4-meter spectrometer measured the backward stimulated Raman scattered light.

\subsection{Thomson-scattering instrumentation}

Two lenses were used to collect Thomson scattered light from the same $70 \mu \mathrm{m} x$ $70 \mu \mathrm{m}$ x $60 \mu \mathrm{m}$ volume in the plasma (Fig. 5.11a). The probe beam ran parallel to the target surface and was focused to a $60 \mu \mathrm{m}$ diameter spot (see Sec. 5.2.1). The angle between the interaction beam and the probe beam was $48^{\circ}$ (Fig. 5.11b). The two Thomson-scattering diagnostics observed perpendicular wave-vectors. The "thermal" Thomson-scattering diagnostic observes acoustic waves that propagate perpendicular to the interaction beam and parallel to the target surface $\left(\mathrm{k}_{\perp}\right.$. The "driven" Thomson-scattering diagnostic observes acoustic waves propagating along the interaction beam axis $\left(\mathrm{k}_{\|}\right)$. When the interaction beam is employed, one of these waves observed by the driven Thomson-scattering diagnostic is excited by stimulated Brillouin scattering (the $+k_{\|}=2 k_{o}$ vector that is co-propagating with the interaction beam).

\subsection{1 “Thermal" Thomson scattering $\left(\mathrm{k}_{\perp}\right)$}

A lens was mounted to collect light scattered by ion-acoustic fluctuations propagating parallel to the target surface and perpendicular to the interaction beam. The angle 

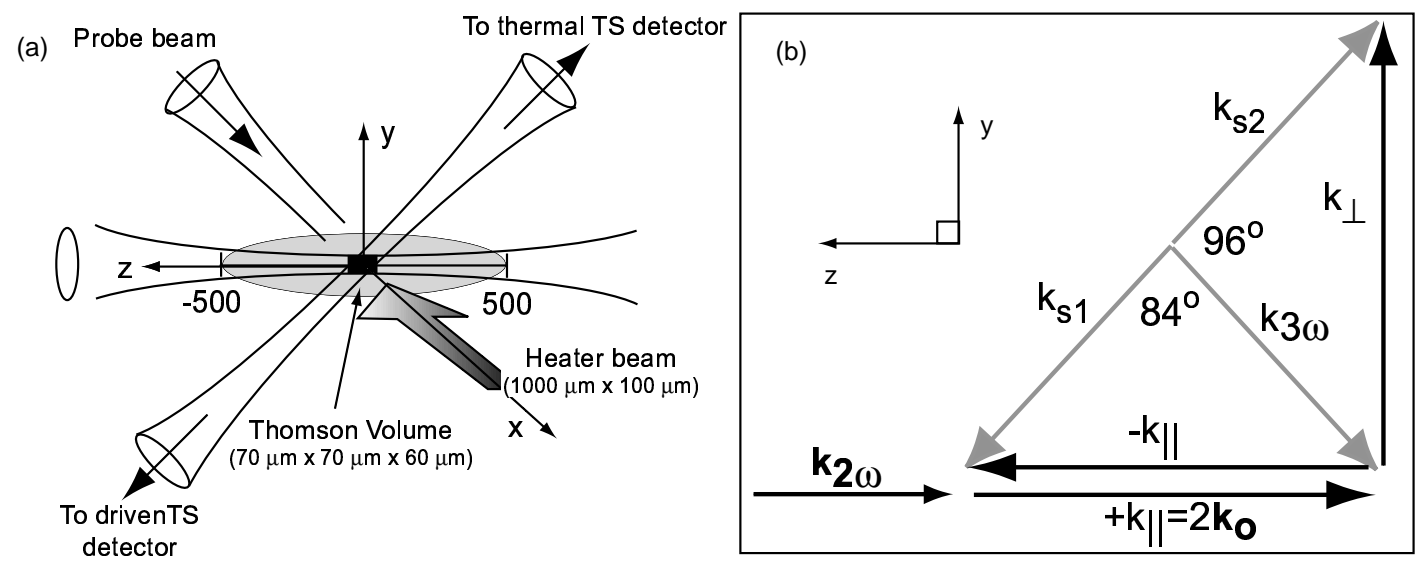

Figure 5.11: (a) The experimental setup is shown with three beams intersecting the Thomson volume at the target chamber center. Light is collected by two $\mathrm{f} / 5$ collection lenses. (b) A vector diagram is shown where one Thomson diagnostic observes the $2 \mathbf{k}_{\mathbf{o}}$ wave vector propagating parallel to the interaction beam and the second Thomson diagnostic observes a wave vector perpendicular to the first.

between the collection lens and the probe beam was $96^{\circ}$ [Fig. 5.11] (see Sec. 4.2). The scattered light was collected and collimated using a $100 \mathrm{~mm}$ focal length $f / 5$ lens. The light was focused onto a $200 \mu \mathrm{m}$ slit of a 1.5 -meter spectrometer by a $25 \mathrm{~cm}$ focal length $f / 5$ achromatic lens. This optical system created a magnification of 3:1. The Thomson-scattering volume was approximately $70 \mu \mathrm{m} \times 70 \mu \mathrm{m} \times 60 \mu \mathrm{m}$ and is defined by the projection of the spectrometer and streak camera slits into the plasma and the beam waist of the focused probe beam.

\subsection{2 "Driven" Thomson scattering $\left(k_{\|}\right)$}

A second identical Thomson-scattering lens system was mounted $\left(42^{\circ}\right.$ down from the axis of the interaction beam) to collect light scattered from ion-acoustic waves with a wave-vector $\left(k_{\|}\right)$parallel to the axis of the interaction beam (Sec.5.5.1) and perpendicular to the wave-vector observed by the thermal Thomson-scattering instrumentation (Fig. 5.11b). 
The angle between the probe beam and scattered light wave vectors was $84^{\circ}$ (see Sec. 4.4). The collected light was focused onto a $200 \mu \mathrm{m}$ slit of a 1-meter imaging spectrometer. This system images the same volume and location in the plasma as the thermal Thomsonscattering diagnostic allowing, for the direct comparison of the two perpendicular wave vectors. 


\section{Chapter 6}

\section{Plasma Characterization}

In this chapter we will discuss the measured plasma conditions. Thomson scattering allows us to measure the electron temperature profiles both parallel and perpendicular to the target surface. We are also able to measure the particle flow parallel to the target surface. Furthermore, the density profile normal to the target is inferred from stimulated Raman measurements.

\subsection{Target configuration}

The targets consisted of pure $\mathrm{Au}$, pure $\mathrm{Be}$, or a mixture of Be with $1 \%, 5 \%$ or $10 \% \mathrm{Au}$ by atomic weight. Targets were $500 \mu \mathrm{m}$ tall $\times 50 \mu \mathrm{m}$ thick. The plasma length was determined by varying the target length between $250 \mu \mathrm{m}$ and $1000 \mu \mathrm{m}$ in the direction of the long axis (1-mm) of the heater beams focal spot. The multi-ion-species targets were fabricated by coating a substrate with alternating Au-Be layers of varying thickness. LASNEX [29], a hydrodynamic code using an atomic physics model that has been tested in 
Refs. [30, 31], calculates that plasmas created with this target configuration homogeneously mix within the first nanosecond of the experiment [19].

\subsection{Electron density}

The light scattered into the full aperture backscatter station (FABS) allows a measure of the electron density. The frequency of the light backscattered by the electron plasma wave (high-frequency electron fluctuations) is a strong function of the electron plasma frequency $\left(\omega_{p e}\right)$ and therefore the density,

$$
\omega=\sqrt{\left(\frac{n_{e} e^{2}}{\epsilon_{o} m_{e}}\right)+\frac{3 k^{2} k_{b} T_{e}}{m_{e}}}
$$

This backscattered high-frequency light undergoes stimulated Raman scattering (SRS), an amplification process analogous to stimulated Brillouin scattering [32, 33, 34, $35,36]$. The spectrum of the SRS light was measured using a 1/4-meter spectrometer (Sec. 5.3.1). Figure 6.1a shows the measured SRS spectrum for a shot where the interaction beam was focused $\mathrm{x}=400 \mu \mathrm{m}$ from the target surface. The spectrum peaks at $665 \mathrm{~nm}$ which corresponds to a density of $\mathrm{n}_{e}(x=400 \mu \mathrm{m})=1.2 \times 10^{20} \mathrm{~cm}^{-3}$. The spectra was fit using a linear gain calculation. To match the spectral width a $10 \%$ density gradient was assumed over $300 \mu \mathrm{m}$. The corresponding SRS gain was $\mathrm{G}_{s b s}=10$. The electron density profile normal to the target surface is shown in Fig. 6.1b. 

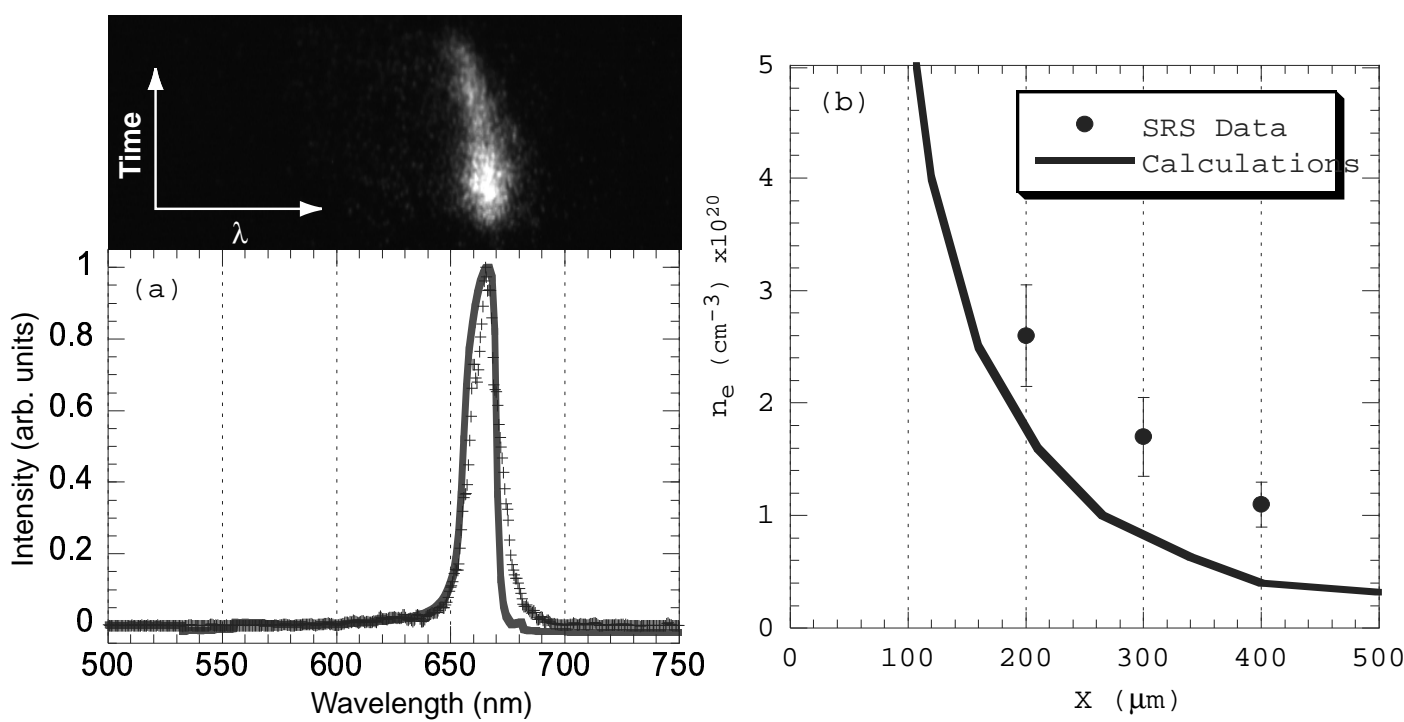

Figure 6.1: (a) The measured SRS spectrum is measured at $\mathrm{x}=400$ microns from the target surface. The peak frequency is $665 \mathrm{~nm}$. The line out is averaged over a 50 ps region, 500 ps after the interaction beam is turned on. (b) The electron density profile is plotted as a function of distance from the plasma surface, $\mathrm{x}$. A LASNEX calculation is shown.

\subsection{Average charge state $(\mathrm{Z})$}

Figure 6.2 shows the thermal Thomson-scattering spectra, measured without the interaction beam, for Be plasmas with 1\%,5\%, and $10 \% \mathrm{Au}$ at a distance of $300 \mu \mathrm{m}$ from the target. The two ion-acoustic modes result in four Thomson-scattering peaks $(2$ modes, counter and co-propagating to the interaction beam axis). The peaks with the greatest separation correspond to a fast ion-acoustic mode. The smaller, less shifted peaks correspond to a slow ion-acoustic mode. Four peaks can be seen, except for the $1 \%$ Au case where the two inner peaks are not resolved by the spectrometer. Indeed the solution to the multi-ion-species dispersion relation shows, for our conditions, the presence of a weakly damped fast mode (the normalized Landau damping is $\nu_{L} / \omega_{a}=0.05$ see Sec. 2.3) and a slow mode with a larger damping $\left(\nu_{L} / \omega_{a}=0.3\right)$. The separation between the peaks 

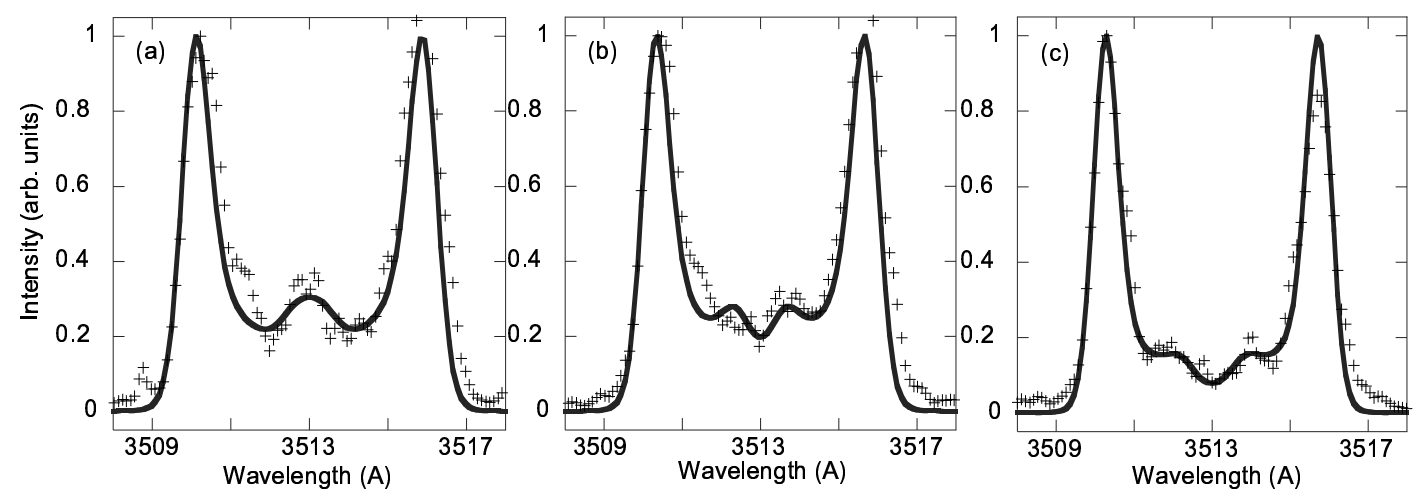

Figure 6.2: Thomson-scattering spectra for three different Au concentrations averaged over $\sim 25$ ps are shown. (a) $1 \% \mathrm{Au}$ (b) $5 \% \mathrm{Au}$ (c) $10 \% \mathrm{Au}$. The solid line represents the best fit theoretical multi-ion-species form factor.

corresponding to a particular mode (see Sec. 2.2.2) is roughly proportional to the phase velocity of the mode and therefore, if the charge state of one of the species is known then the electron temperature and charge state of the second species can be measured.

The Thomson-scattering spectra were fit using the standard multi-ion-species form factor, Eq. 4.12. For these conditions, Be is fully ionized $Z_{B e}=4$. Therefore, the electron temperature is determined by the outer peaks $\left(T_{e}(\mathrm{x}=300 \mu \mathrm{m})=600 \mathrm{eV}\right)$, while the average charge state is determined through a fit of the inner peaks $\left(Z_{A u}(\mathrm{x}=300 \mu m)=40\right)$.

\subsection{Plasma electron temperature}

\subsection{1 $T_{e}$ normal to target surface}

Figure 6.3a shows Thomson-scattering data for pure Au plasmas at various distances from the target. The product of the Au charge state and electron temperature, $Z_{A u} T_{e}$, is measured along the heater beam axis by varying the distance of the Thomsonscattering volume from the Au disk targets. Figure $6.3 \mathrm{c}$ is a plot of the electron temperature 
(a)

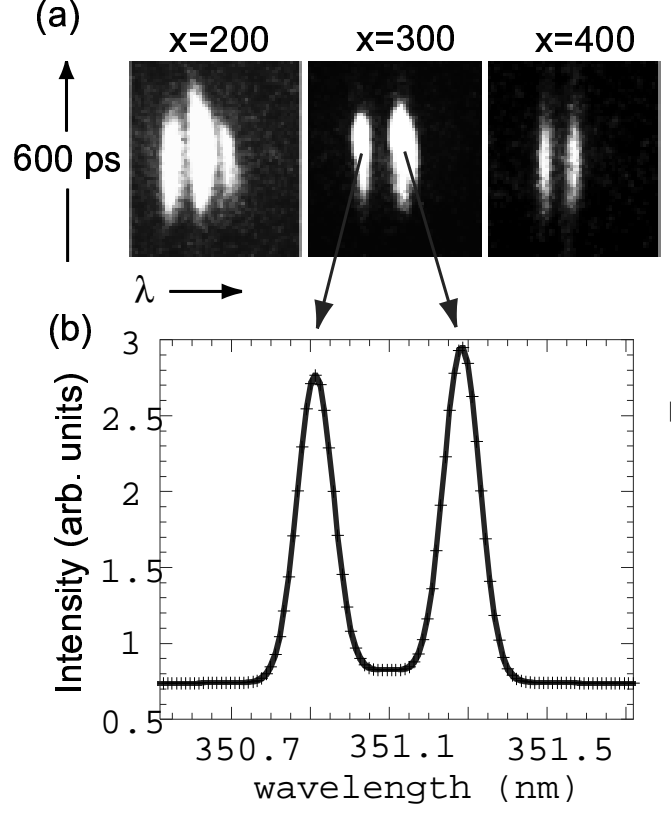

(c)

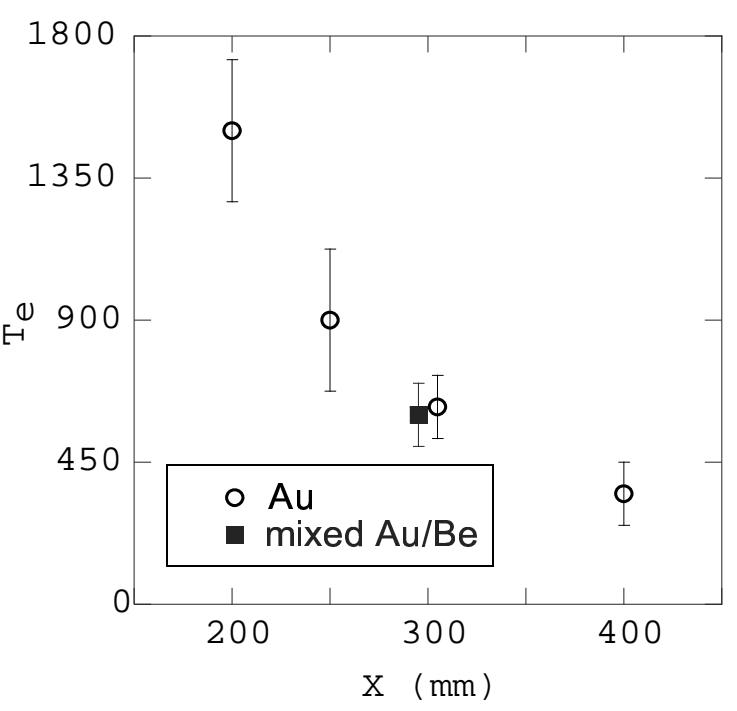

Figure 6.3: (a) Gold Thomson-scattering streak data are shown for three separate distances from the target. (b) A $50 \mathrm{ps}$ lineout is shown for the above Au streak data. (c) The electron temperature is plotted as a function of the distance from the target surface at a time of 1.4 ns.

gradient measured along the $\mathrm{x}$-axis using an average $\mathrm{Au}$ charge state of $\mathrm{Z}_{A u}=43$ which was measured using multi-ion-species plasmas at $\mathrm{x}=300 \mu \mathrm{m}$ (Section 6.3). The scattering spectra for the closest measurement to the target $(\mathrm{x}=200 \mu \mathrm{m})$ shows three distinct peaks; if one assumes that the middle peak is stray light scattered directly from the target surface, then $T_{e}=1500 \mathrm{eV}$. If on contrary we interpret either one of the outer peaks as spurious, the inferred electron temperature will be $400 \mathrm{eV}$.

\subsection{2 $T_{e}$ parallel to the interaction beam axis}

The data for Be plasmas shown in Figure 6.4 indicates a constant 450 eV electron temperature parallel to the interaction beam axis at a distance of $400 \mu \mathrm{m}$ from the target 


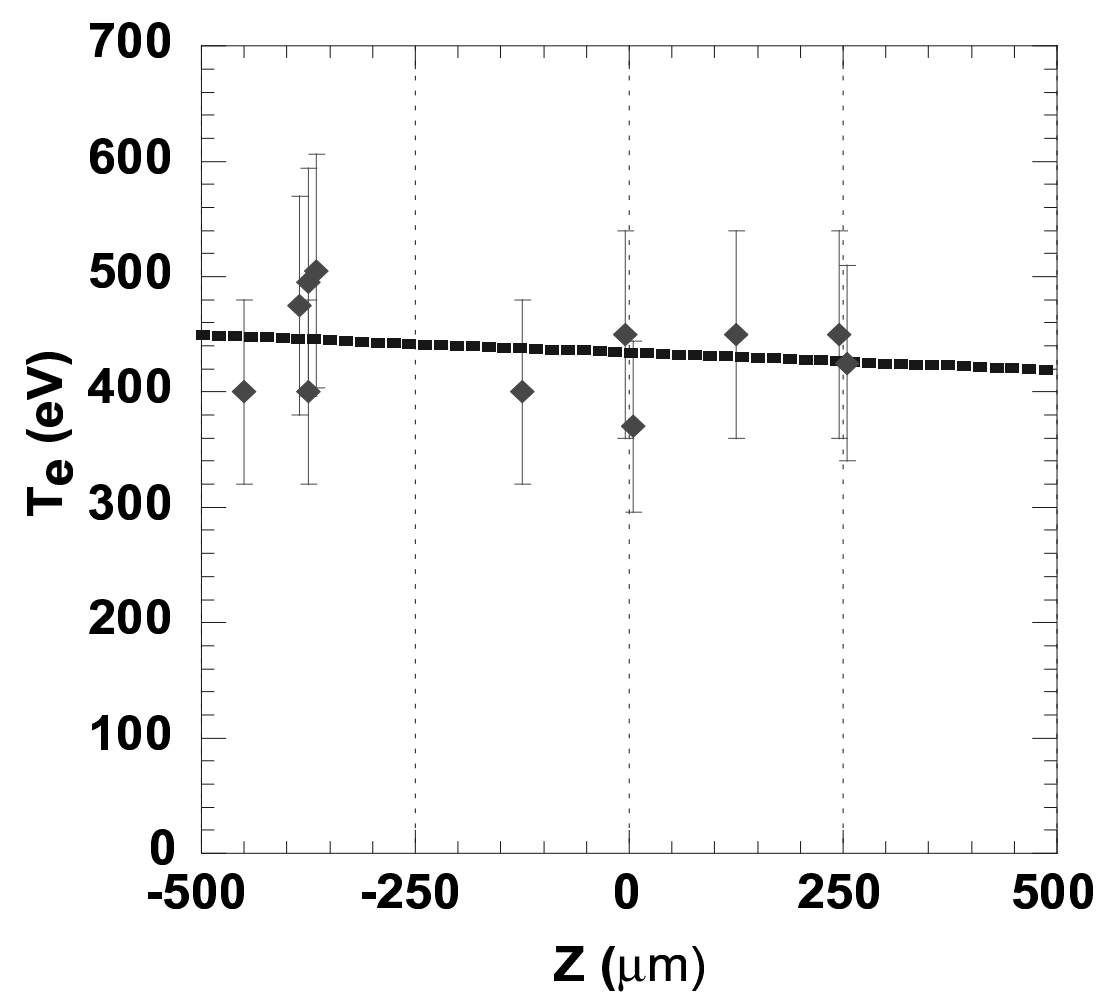

Figure 6.4: The electron temperature profile parallel to the interaction beam axis is shown to be constant. A best fit line is plotted.

surface. This temperature profile was measured using the thermal Thomson-scattering diagnostic (See Sec. 5.5.1), which observes wave numbers perpendicular to the interaction beam $\left(\mathrm{k}_{\perp}\right)$.

\subsection{Plasma ion temperature}

The two-ion-species plasmas have further allowed us to measure the ion temperature in a unique way; the relative amplitude of the peaks in the Thomson-scattering spectra belonging to the slow and fast ion-acoustic waves provide an accurate measure of the ion temperature, $T_{i}(\mathrm{x}=300 \mu \mathrm{m})=250 \mathrm{eV}$. As a result of the sensitivity of Landau damping 
to ion temperature, the damping of the fast mode increases while the damping of the slow mode decreases when the ion temperature is increased (see Sec. 2.5). This is illustrated by a respective increase or decrease in the amplitude of the peaks in the Thomson-scattering spectra (see Sec. 4.3) and allows us to use the ion temperature as a sensitive fit parameter.

\subsection{Plasma flow parallel to target surface}

When observing low frequency collective effects with Thomson scattering, the frequency of the scattered light is shifted from the laser frequency in two ways: by the frequency of the ion-acoustic wave in the frame of the plasma $\left(\omega_{a}=c_{s} k_{a}\right)$ and by the relative ion flow in the laboratory frame $\left(\mathrm{v}_{f}\right)$. See Chapter 4 . In our case, we are able to measure the velocity flow along the interaction beam axis by measuring the light that has been blueshifted by acoustic waves counter-propagating in the direction of the interaction beam. Because we employ two Thomson-scattering diagnostics that observe the same volume in the plasma, we are able to measure the local sound speed $\left(\mathrm{c}_{s}\right)$ with the Thermal ThomsonScattering Diagnostic $\left(\mathrm{k}_{\perp}\right)$ and the velocity flow $\left(\mathrm{v}_{f}\right)$ with the Driven Thomson-Scattering Diagnostic $\left(\mathrm{k}_{\|}\right)$.

Figure 6.5 plots the peak wavelength of the light scattered from thermal ionacoustic waves $\left(-\mathrm{k}_{||}\right)$. The fact that we have independently measured the local sound speed (Fig. 4.2) enables us to know the frequency shift of the scattered light due to the ion-acoustic waves in the frame of the plasma (Eq. 4.3). This frequency shift corresponds to $\Delta \lambda_{\text {iaw }}=2.5$ $\AA$; therefore, we fit a $200 \mu \mathrm{m}$ velocity plateau in the middle of the plasma (Fig. 6.5). The additional shift in wavelength as one moves away from the plateau $\left(\Delta \lambda_{\text {flow }}\right)$ is a result of 


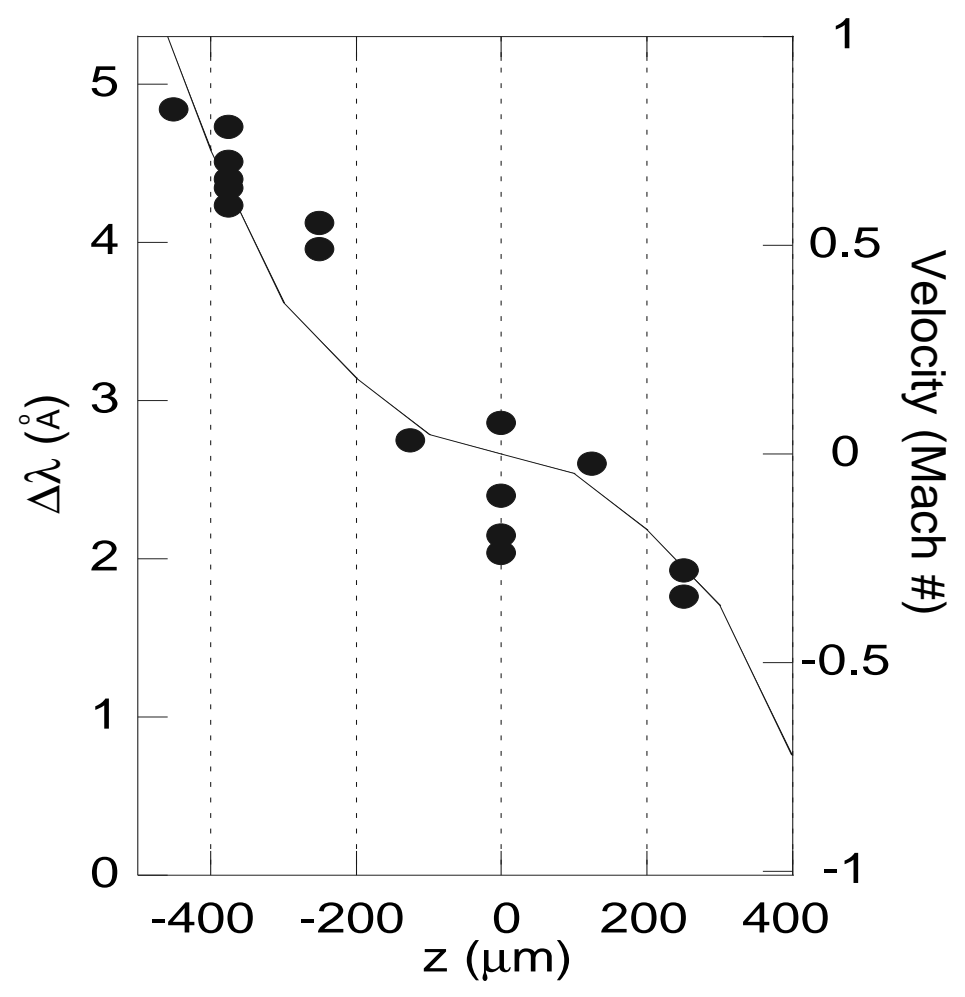

Figure 6.5: Light scattered from ion-acoustic waves counter-propagating to the interaction beam axis allow the measure of a velocity gradient along the interaction beam axis. The blue-shifted light is collected by the Driven Thomson Scattering Diagnostic. A Mach 1 flow is measured at the edge of the plasma.

the relative ion flow in the laboratory frame (Eq.6.4). 


\section{Part III}

\section{Results and Discussion}




\section{Chapter 7}

\section{The effects of velocity gradients on}

\section{SBS}

Stimulated Brillouin scattering (SBS) is a result of the resonant ponderomotive coupling of an incident light wave, a reflected light wave, and an ion-acoustic wave. This resonance can drive ion-acoustic waves to large amplitudes. In principle, this process could result in the reflection of a large fraction of the incident energy for inertial confinement fusion (ICF) targets. In order to minimize this energy loss, a good understanding of mechanisms that could limit SBS is important. In many targets, the velocity gradient within the expanding plasma provides such a mechanism; the frequency of the local acoustic waves is Doppler shifted, therefore, detuning the three-wave resonant process [37].

In this chapter, we present the first direct measurement of SBS detuning by a velocity gradient. A novel use of two Thomson-scattering diagnostics has allowed us to directly measure the frequency and amplitude of the ion-acoustic wave responsible for SBS 
as a function of space. This is the first measurement that spatially resolves both the frequency and the amplitude of the ion-acoustic waves directly responsible for SBS. These measurements link the saturation of the SBS instability to the frequency detuning from an expanding plasma. We have independently measured the ion-acoustic frequency and the frequency of the driven acoustic wave at various positions in the plasma. Therefore, by comparing the local ion-acoustic frequency, with the local frequency of the driven acoustic wave we have measured the actual detuning of the SBS instability. The measured electron temperature, velocity gradient, and electron density have been included in fluid simulations which clearly support the measured SBS detuning by a velocity gradient.

\subsection{Experimental setup}

The experiments used a three-beam configuration at the Trident Laser Facility (see Chapt. II). The Be plasmas were produced by a heater beam with $180 \mathrm{~J}$ of $2 \omega(\lambda=527 \mathrm{~nm})$ laser light in a 1.2-ns-long square pulse. The heater beam was focused normal to the target surface using an $f / 6$ lens and a strip line random phase plate (Fig. 7.1c). This produced a line focus with an intensity of $10^{14} \mathrm{~W} \mathrm{~cm}^{-2}$ and an initial $1000 \mu \mathrm{m} \times 100 \mu \mathrm{m}$ Be plasma [27]. An interaction beam ( $50 \mathrm{~J}, 2 \omega, 1.2$-ns-long square pulse) was aligned $\mathrm{x}=400 \mu \mathrm{m}$ from the target surface and was focused to a $60 \mu \mathrm{m}$ diameter spot, resulting in an intensity of $1.5 \times 10^{15} \mathrm{~W} \mathrm{~cm}^{-2}$ (see Sec. 5.2.1). The interaction beam was used to drive SBS which excites ion-acoustic waves with wave-vector $\mathbf{k}_{||}=2 \mathbf{k}_{\mathbf{o}}$ co-propagating in the direction of the interaction beam. The backscattered SBS light was collected and collimated by the focusing lens of the interaction beam. A fraction of the backscattered light was focused on to a fast 


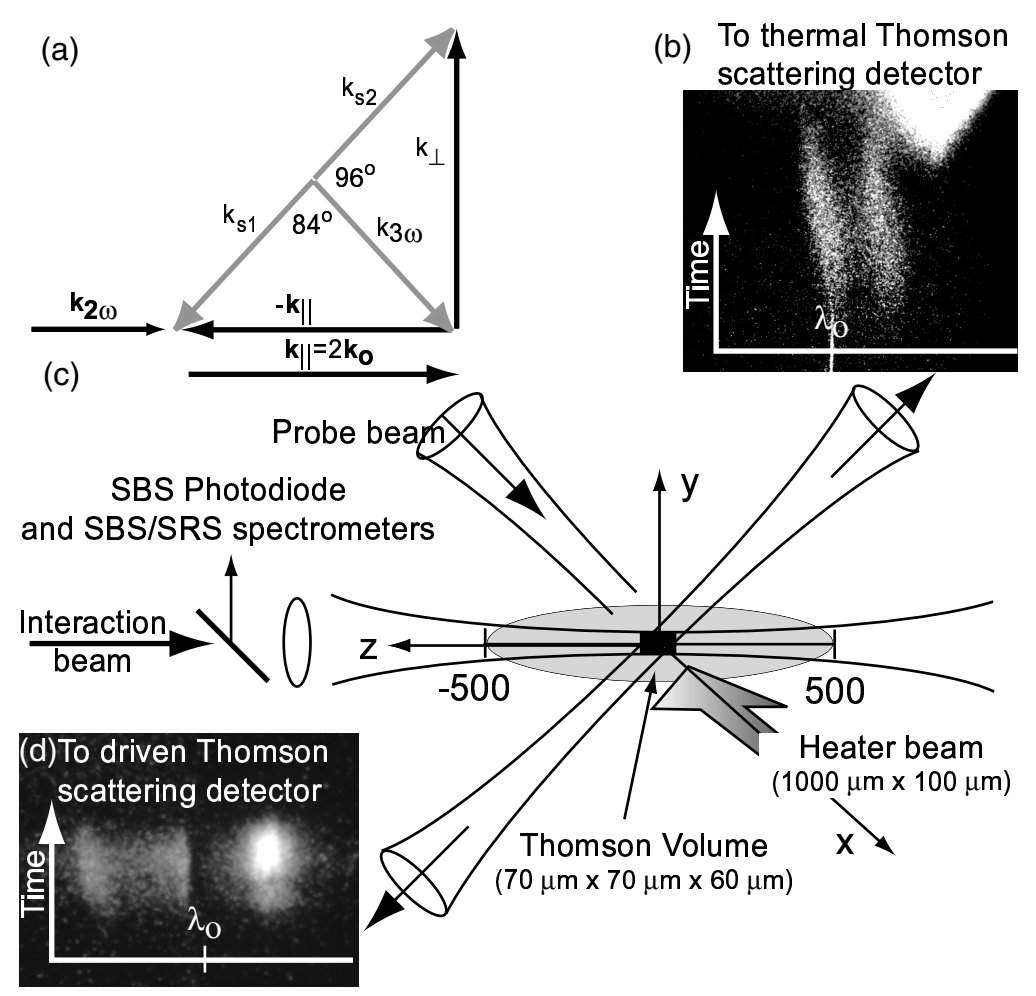

Figure 7.1: (a) A diagram detailing the ion-acoustic wave-vectors probed is shown in the top left corner. (b) A thermal Thomson-scattering spectrum $\left(\mathrm{k}_{\perp}\right)$ and $(\mathrm{d})$ a driven Thomsonscattering spectrum $\left(\mathrm{k}_{\|}\right)$are shown. (c) The experimental setup is shown.

photodiode and two, 1/4-meter SRS and 1-meter SBS, spectrometers. The SRS and SBS spectra were measured with a respective resolution of $0.5 \mathrm{~nm}$ and $0.45 \AA$. The absolute SBS energy was measured with the diode (see Sec. 5.4) [38].

The third laser beam, $3 \omega(\lambda=351 \mathrm{~nm})$, was used as a Thomson probe beam. The probe beam and Thomson-scattering collection optics define a volume $(70 \mu \mathrm{m} \times 70 \mu \mathrm{m} \times 60 \mu \mathrm{m})$ located at the center of the chamber (see Sec. 5.5). Light scattered from the Thomsonscattering volume was imaged onto two separate Thomson-scattering spectrometers. The interaction beam is focused in the middle of this volume. The first Thomson-scattering diagnostic probes ion-acoustic fluctuations (Fig. 7.1b) propagating perpendicular to the in- 
teraction beam $\left(\mathbf{k}=\mathbf{k}_{\perp}\right)$ and allows us to independently measure the electron temperature $T_{e}$. The geometry (Fig. 7.1a) of the second Thomson-scattering diagnostic was chosen to measure light scattered from SBS-driven ion-acoustic waves (Fig. 7.1d) that are excited by the SBS instability $\left(\mathbf{k}=\mathbf{k}_{\|}\right)[39]$.

The interaction beam was timed to turn on $1.0 \mathrm{~ns}$ after the heater beam. The 180 ps Gaussian Thomson probe beam was turned on 400 ps after the interaction beam near the peak of the backscattered SBS light (see Sec. 5.2.3).

\subsection{Plasma characterization}

The plasma has been well-characterized (see Sec. 6); Fig. 7.2a shows a constant electron temperature profile $\left(\mathrm{T}_{e}=450 \mathrm{eV}\right)$ along the interaction beam axis. The location of the Thomson-scattering volume and the focus of the interaction beam remained at TCC throughout the experiment; the target (and the heater beam) was moved to probe different positions in the plasma parallel to, and $400 \mu \mathrm{m}$ from, the surface of the target.

Figure $7.2 \mathrm{~b}$ shows a Thomson-scattering spectrum taken from the streak data in Fig. 7.1b. Two peaks corresponding to ion-acoustic fluctuations propagating along the direction of $\mathbf{k}_{\perp}$ are evident. Fitting the data using a standard theoretical form factor [23] gives the electron temperature (see Chapt. 4). The measured peaks are broadened by velocity gradients perpendicular to the interaction beam. This broadening is to be expected as the initial plasma length is only $\mathrm{y}=100 \mu \mathrm{m}$ in the perpendicular direction; this Thomsonscattering diagnostic probes the plasma expanding in the perpendicular direction. The measured width of the peaks $(\delta \lambda)$ gives an estimated velocity range $\frac{\delta v}{v}\left(\propto \frac{\delta \lambda}{\lambda}\right)=0.5$ within 

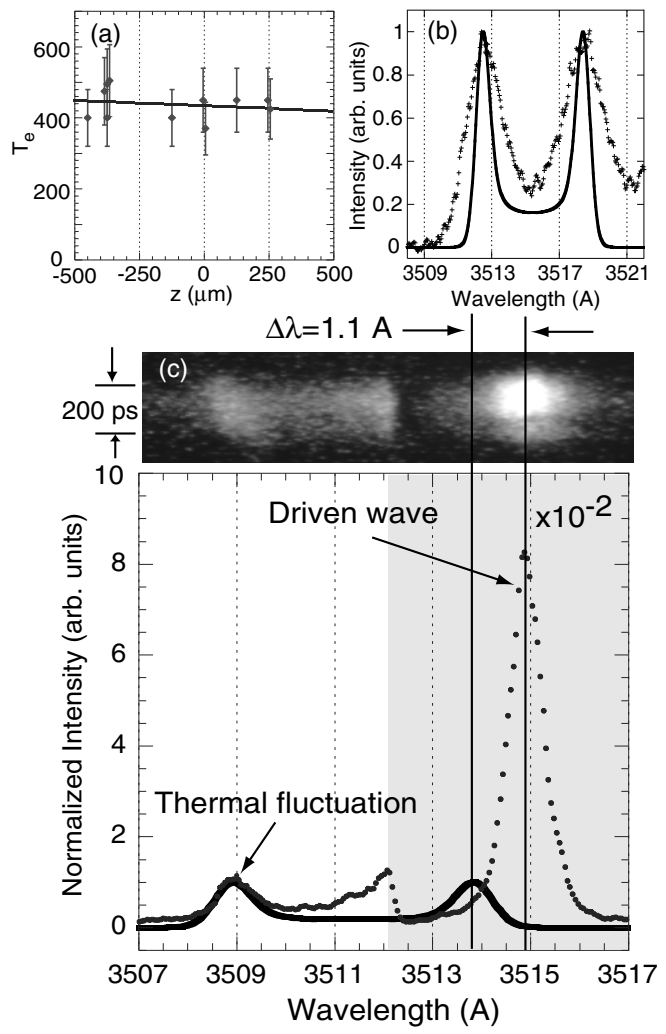

Figure 7.2: (a) A constant electron temperature, $T_{e}=450 \mathrm{eV}$, is measured along the interaction beam axis. (b) Representative Thomson-scattering spectra taken from the spectra in Fig. 7.1b where light is scattered from ion-acoustic fluctuations propagating perpendicular to the interaction beam $\left(\mathbf{k}_{\perp}\right)$. (c) Thomson-scattering data are shown collected by the diagnostic observing ion-acoustic waves propagating parallel to the interaction beam $\left(\mathbf{k}_{\|}\right)$. The spectra (points) is shown below averaged over 50 ps. The theoretical form factor (solid line) is fit to the blue-shifted light using a velocity flow $\frac{v}{v_{t h}}=0.7$ and the electron temperature measured with the first Thomson-scattering diagnostic $\left(T_{e}=450 \mathrm{eV}\right)$. The shaded region was optically filtered by x100. 
the Thomson-scattering volume.

The blue-shifted intensity peak in the spectra mesured by the second Thomsonscattering diagnostic is a direct measure of the local resonant frequency of the ion-acoustic waves in the laboratory frame $\left(-\mathbf{k}_{\|}\right)$. The frequency of the scattered light in the laboratory frame is shifted from the laser frequency in two ways: by the frequency of the probed ion-acoustic wave (function of the electron temperature), and by the overall flow of the plasma relative to the frame of the laboratory (Doppler shift along $\mathrm{k}_{\|}$). Since the electron temperature is known, the plasma flow parallel to the z-axis is determined from the local frequency shift between the frequency of the Thomson-scattering probe $\left(\lambda_{3 \omega}=351.3 \mathrm{~nm}\right)$ and the blue-shifted intensity peak.

Figure 7.2c shows a Thomson-scattering spectra in which two intensity peaks can be observed. The blue-shifted intensity peak is fit using the independently measured electron temperature, and a flow of $\frac{v}{c_{s}}=0.7$ is measured. Note that the use of two Thomsonscattering diagnostics is necessary as the SBS-driven acoustic wave is not assumed to be at the local resonant ion-acoustic frequency. This is evident in Fig. 7.2c, where the red-shifted intensity peak and the corresponding resonant intensity peak are offset by $1.1 \AA$.

Figure 7.3d shows the plasma flow along the z-axis (see Sec. 6.6) measured from the frequency of the local resonant ion-acoustic fluctuations $\left(-\mathbf{k}_{\|}\right)$. A $200 \mu \mathrm{m}$ velocity plateau is measured in the center of the plasma; a Mach 1 flow was measured in the front edge of the plasma. One can directly observe the effect of the velocity gradient on SBS from the frequency mismatch between the measured frequency of the SBS driven acoustic wave, and the measured frequency of the local resonant waves. 


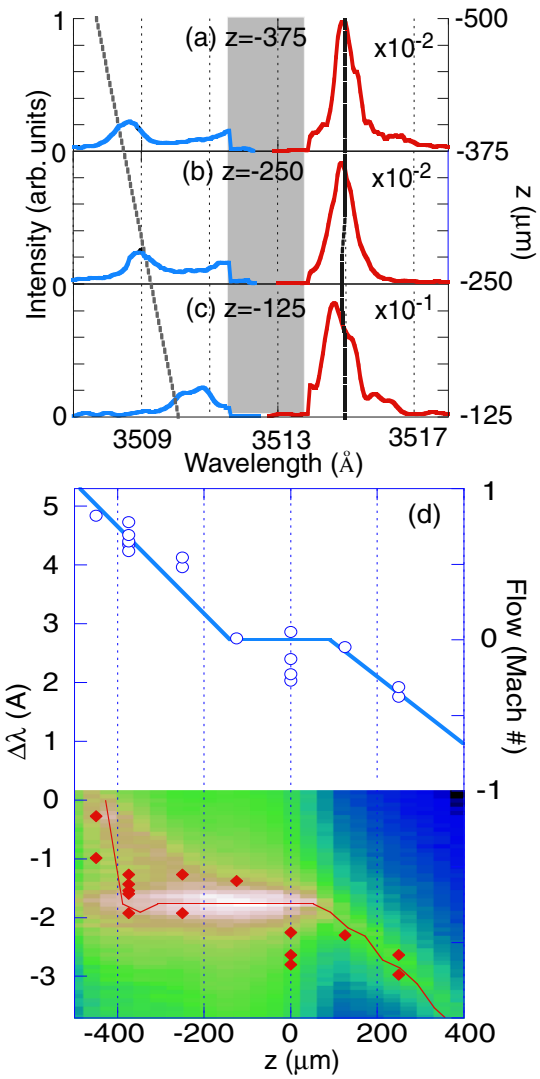

Figure 7.3: (color). Intensity spectra at $\mathrm{z}=(\mathrm{a})-375 \mu \mathrm{m}$, (b) $-250 \mu \mathrm{m}$, and (c) $-125 \mu \mathrm{m}$ show a velocity gradient. Furthermore, the frequency of the SBS driven ion-acoustic wave is unchanged. The shaded area has been filtered by a factor of 10. (d) The wavelength shift for red-shifted (solid squares) and the blue-shifted (open circles) intensity peaks are plotted. The blue-shifted intensity peaks reveal a Mach 1 flow parallel to the interaction beam (right axis). A pF3d simulation is shown where large acoustic waves (color scale $=\delta \mathrm{n} / \mathrm{n}_{e}$ ) are driven in the plateau region. 


\subsection{Detuning by a velocity gradient}

Figure 7.3 shows spectra for representative data shots at three positions in the plasma; the frequency of the driven ion-acoustic wave remains unchanged $(\lambda=3515 \AA)$ while the frequency of the local resonant ion-acoustic waves $\left(-\mathbf{k}_{\|}\right)$increase as one moves from the plateau to the front of the plasma (this increase is a direct effect of the plasma flow as the electron temperature is constant). SBS light is scattered from the velocity plateau where large resonant acoustic waves are driven. The light scattered from the plateau and the incident light create a constant ponderomotive force with a constant frequency profile in the front of the plasma. This constant frequency ponderomotive force drives non-resonant acoustic waves (i.e., the velocity gradient shifts the resonant frequency from the constant frequency of the ponderomotive force).

The experiment was simulated in 2-D using pF3d [40]. A f/6 RPP beam with a best focus of $60 \mu \mathrm{m}$ and an average vacuum intensity of $10^{15} \mathrm{~W} \mathrm{~cm}^{-2}$ was propagated through a $1000 \mu \mathrm{m}$ slab of plasma $\left(T_{e}=450 \mathrm{eV}, n_{e}=1 \times 10^{20} \mathrm{~cm}^{-3}\right)$. A Mach 2 transverse flow (lateral expansion of the target) and the measured velocity profile along the propagation axis shown in Fig. 7.3d were included.

Figure $7.4 \mathrm{a}$ shows a plot of the interaction beam 70 ps into the simulation. Intensity of the most intense speckles is around $4 \times 10^{15} \mathrm{~W} \mathrm{~cm}^{-2}$. A linear non-local heat transport model was used, but had only a small effect on the results. The maximum local temperature fluctuation observed was $\frac{\Delta T}{T} \approx 30 \%$ in the most intense speckles. The transverse flow suppressed most of the filamentation and the simulations show little beam bending (Fig. 7.4a). 


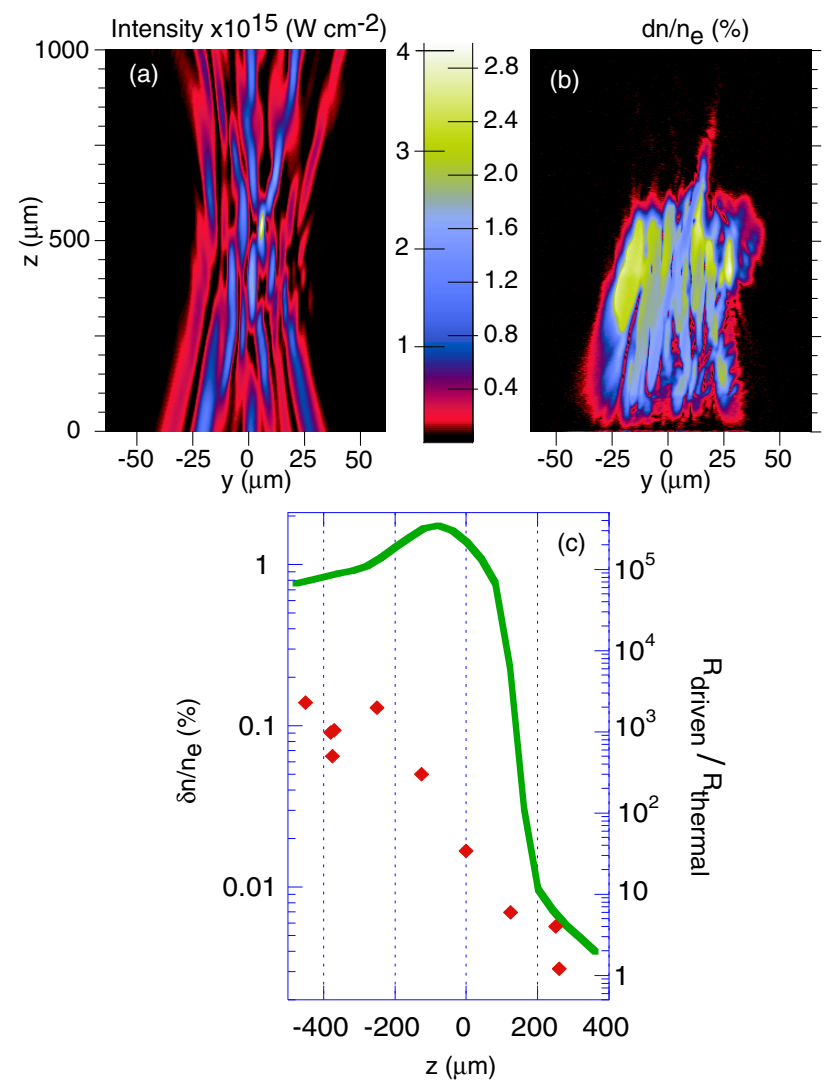

Figure 7.4: (color). (a) The intensity of the interaction beam is plotted 70 ps into the pF3d simulation. (b) The ion-acoustic waves are large in the front of the plasma. (c) The measured amplitude of the excited ion-acoustic wave (squares) is plotted as a function of the position along the interaction beam. The amplitude profile given by pF3d is also plotted (solid). 
Figure 7.4b and the solid line in Fig. 7.4c show that large ion-acoustic waves are driven by SBS only in the front half of the plasma [41]. The velocity gradient in the back of the plasma prevents any significant SBS growth. The plateau in the middle provides a resonant region where SBS drives a large acoustic wave. The amplitude of the driven acoustic wave saturates near the front of the velocity plateau. In the front of the plasma, the strong velocity gradient prevents SBS growth at the local resonant frequency but the reflected light coming from the plateau and the incoming light provide a constant frequency ponderomotive force that drives non-resonant acoustic waves. The off-resonant acoustic waves are clearly observed in Fig 7.3d. As one moves away from the plateau and towards the front of the plasma, the acoustic wave amplitude slightly decreases as the ponderomotive driver is increasingly off resonance (Fig. 7.4c).

The effect of detuning SBS by a velocity gradient is further verified by post processing the simulation providing a measure of the local frequency of the driven ion-acoustic waves. This agrees well with the experimentally measured frequencies, as shown in Fig. 7.3d; off-resonant driven ion-acoustic waves are evident in the front part of the plasma.

While the amplitude of driven acoustic waves $\left(\frac{\delta n}{n_{e}}\right)$ is readily obtained from simulations, it has to be calculated from the intensity peaks in the Thomson-scattering spectra. This is done by comparing the scattered reflectivity from thermal fluctuations (i.e. the power scattered in to the left intensity peak in Fig. 7.2c divided by the incident laser power), which is given by:

$$
R_{\text {thermal }}=\frac{1}{4} r_{0}^{2} n_{e} L \Delta \Omega_{\text {exp }}
$$

where $r_{0}$ is the classical electron radius, $\mathrm{L}$ is the length along the direction of the $3 \omega$ probe 
beam, and $\Delta \Omega_{e x p}$ is the solid angle of the collecting optics (an $\mathrm{f} / 5$ lens), with the reflectivity scattered by the driven acoustic wave given by:

$$
R_{\text {driven }}=\frac{1}{4} r_{0}^{2} n_{e}^{2} \lambda_{3 \omega}^{2} L L_{c}\left(\delta n / n_{e}\right)^{2}
$$

where $\delta n / n_{e}$ is the amplitude of the acoustic wave and $L_{c}$ its correlation length along the direction of the $3 \omega$ probe beam. $L_{c}$ can be estimated by the transverse size of speckles generated by the interaction beam $f_{2 \omega} \lambda_{2 \omega}$.

Figure $7.4 \mathrm{c}$ shows that this estimation of $\delta n / n_{e}$ reproduces the overall spatial shape predicted by $\mathrm{pF} 3 \mathrm{~d}$, but the absolute amplitude is smaller by almost an order of magnitude. Various factors can explain this discrepancy. For instance, any misalignment of the optics, an overestimation of $L_{c}$, or enhanced thermal fluctuations would lead to an underestimation of the acoustic wave amplitude. Assuming the latter, we have verified that increasing the amplitude of the thermal noise source used both in the Thomson-scattering calculations and in the SBS simulations by an order of magnitude gives a good agreement between the measured and simulated wave amplitude and spatial profiles. A study of the amplitude of the thermal noise in laser plasmas needs to be completed to investigate the discrepancies in the calculated wave amplitudes seen in this and other studies [19].

A peak SBS reflectivity of $6 \%$ was measured. The SBS spectrum (Fig. 7.5) confirms our analysis of the effect of the velocity gradient on SBS. The frequency of the peak intensity is consistent with the frequency of the large off-resonant waves observed by Thomson scattering in the front part of the plasma, and corresponds to the resonant acoustic frequency in the velocity plateau. The spectrum has a tail towards the fundamental frequency that corresponds to light scattered from the flow propagating towards the detector. There 


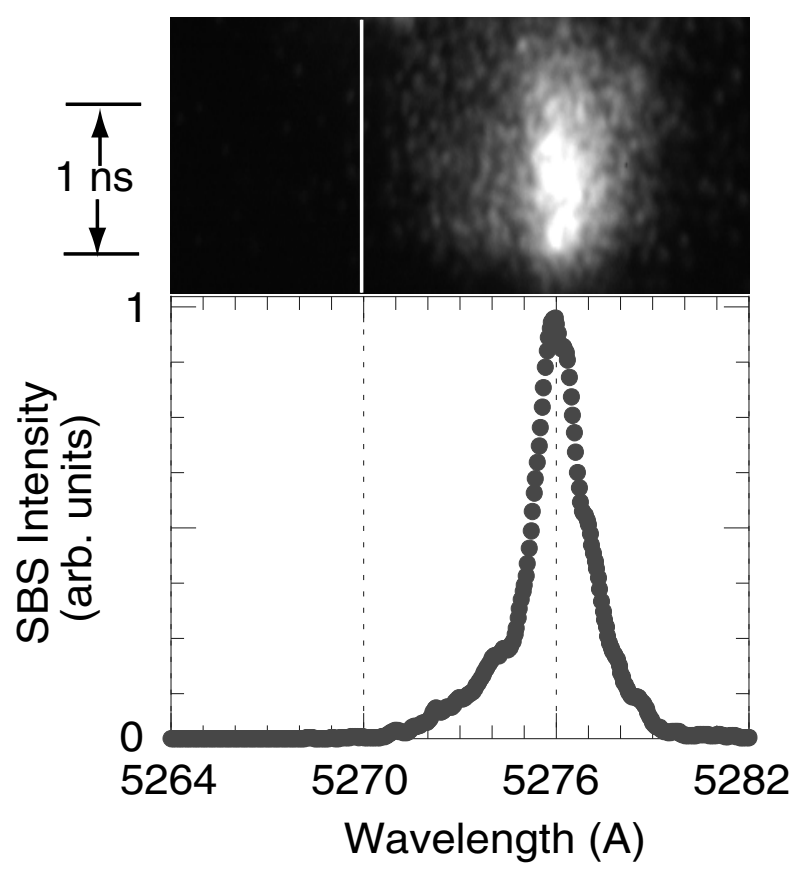

Figure 7.5: Ths SBS spectra is plotted as a function of intensity. The peak intensity corresponds to light scattered from the velocity plateau; a tail towards the fundamental frequency corresponds to light scattered from ion-acoustic waves in the front of the plasma.

is a sharp cutoff towards higher wavelengths indicating that there is no scattering coming from the back of the plasma where the velocity flow is propagating away from the detector. This is consistent with the fact that we measure very small ion-acoustic waves in the back portion of the plasma (Fig. 7.4c). Overall, the SBS spectrum is characteristic of the central plateau, while the expanding plasma only slightly modifies its shape.

\subsection{Chapter summary}

In summary, we have presented the first direct measurement of SBS detuning by a velocity gradient. We studied an expanding plasma with a central velocity plateau. The frequency mismatch between the frequency of the SBS driven ion-acoustic wave and 
the local resonant frequency (as measured from thermal waves) was mapped throughout the plasma. It was found that acoustic waves in the flow are driven off resonance by the ponderomotive force with a beat frequency created by the incoming light and the light reflected from the plateau. This detuning inhibits further SBS growth in the flow. These findings are important to many laser-plasma experiments and occurs in various ICF direct drive [42] and indirect drive targets [19]. We have shown that the SBS spectrum gives insight into the locations of SBS growth, and Thomson scattering on driven ion-acoustic waves provides a direct verification of modeling the effect of a velocity gradient on SBS. 


\section{Chapter 8}

\section{Observation of ion heating by}

\section{stimulated-Brillouin-scattered-}

\section{driven ion-acoustic}

\section{waves}

In this chapter, we present observations of the growth of ion-acoustic waves in a well-characterized multi-ion-species plasma consisting of $\mathrm{Au}$ and Be. Using Thomson scattering, we directly measured the growth of the ion-acoustic waves responsible for the SBS light (see Sec. 4.4). The Thomson-scattering spectra show simultaneously the scattering from thermal ion-acoustic fluctuations and ion-acoustic waves that have been excited to large amplitude by SBS using a $2 \omega$ interaction beam.

For this experiment the addition of small amounts of Au to Be plasmas results in 
a solution to the kinetic dispersion relation with two modes (see Sec. 2.2.2). The mode with the larger phase velocity is weakly damped while the other mode is heavily damped. In this chapter we call the "fast mode" the solution to the kinetic dispersion relation with the larger phase velocity while the "slow mode" is the solution with the smaller phase velocity.

We observe that when exciting SBS, only the fast mode grows to large amplitude. In addition, we use a new technique that measures the relative damping of the two modes to determine the ion temperature with high accuracy (see Sec. 4.3). We measure up to a factor of two increase in the ion temperature when ion-acoustic waves are excited by SBS. This measurement of an increase in ion temperature, and its correlation with SBS, is direct quantitative evidence of hot ions created by trapping in laser plasmas. Motivated by this observed increase in ion temperature and our SBS reflectivity measurements, we developed a model that includes trapping effects (Sec. 3.4), providing a saturation mechanism that gives SBS reflectivity values consistent with the experiment.

\subsection{Thomson scattering on SBS driven ion-acoustic waves}

Figure 8.1a shows the Thomson-scattering data in which ion-acoustic waves are being driven by the ponderomotive force due to the interaction beam. Figure $8.1 \mathrm{~b}$ shows line outs of these data revealing that once the interaction beam is turned on, only the ion-acoustic waves excited by SBS are driven to large amplitudes. The undriven part of the spectra are fit using the standard theoretical form factor (Eq. 4.12); when ion-acoustic waves are excited, the driven part of the spectra is fit with a Gaussian. This fit is used to calculate the relative amplitude of the driven acoustic wave. The ion-acoustic wave 

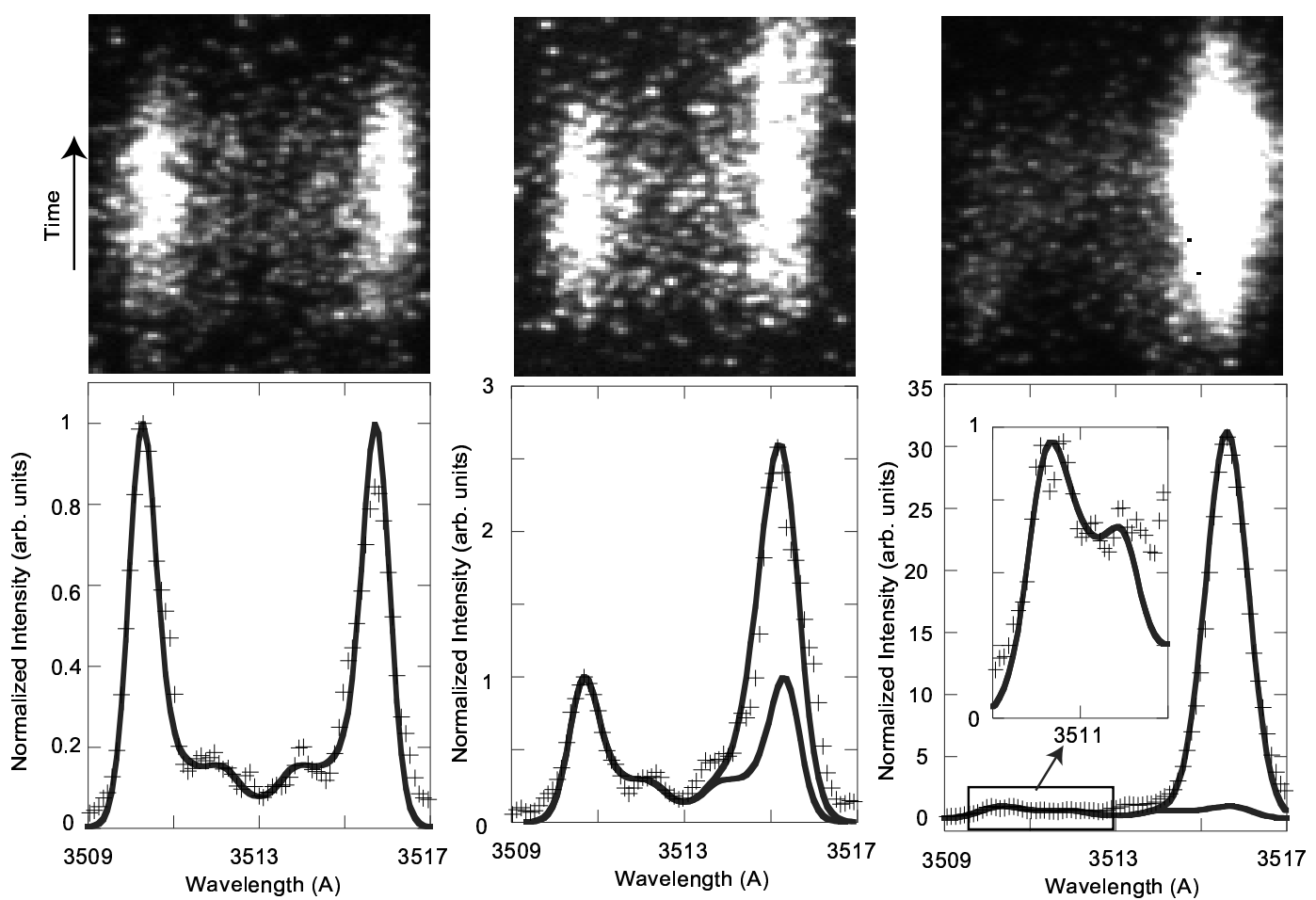

Figure 8.1: (a) Streaked Thomson spectra for a Be plasma with a 10\% Au mixture are shown for a shot without the interaction beam (undriven) and shots with interaction beam intensities of $5 \times 10^{15} \mathrm{~W} \mathrm{~cm}$ cm $^{-2}$ and $7 \times 10^{15} \mathrm{~W} \mathrm{~cm}^{-2}$ (driven). (b) Line outs show the increasing amplitude of the red-shifted light. The blue-shifted peaks are fit with the standard multi-ion-species form factor showing an increase in ion temperature from $250 \mathrm{eV}$ to $475 \mathrm{eV}$. The data was collected using the driven Thomson scattering diagnostic described in Sec. 5.5 and the dts2 spectrometer (see Table 5.3).

amplitude is defined to be the square root of the ratio between the area under the red-shifted Thomson-scattering spectra, and the area under the blue-shifted spectra; the ion-acoustic wave amplitude is proportional to $\delta n / n_{e}$.

Our SBS linear gain calculations (see Sec. 3.3), reproduce the findings of Fig. 8.1 with almost no amplification of the slow mode $\left(G_{S B S}^{\text {slow }} \approx 5\right)$ and a large amplification for the fast mode $\left(G_{S B S}^{\text {fast }} \approx 200\right)$. This difference in the amplification can be explained; when these modes are being driven in steady state, the rate of energy lost through Landau damping 
$\left(\nu_{L} \mathrm{~W}_{a}\right)$ must be balanced with the energy transferred to the electrons by the ponderomotive coupling. The energy of the SBS driven acoustic wave is given by:

$$
W_{a}=\frac{\left|E_{a}\right|^{2}}{16 \pi} \frac{\partial\left(\omega \epsilon_{r}\right)}{\partial \omega} .
$$

For a given electrostatic field $E_{a}$ (i.e. a given electronic density perturbation), the rate of energy lost through Landau damping for the individual modes can be compared,

$$
\frac{\left(\nu_{\text {slow }} W_{a}^{\text {slow }}\right)}{\left(\nu_{\text {fast }} W_{a}^{\text {fast }}\right)} \approx \frac{\nu_{\text {slow }}}{\nu_{\text {fast }}}\left(1+\frac{\operatorname{Re}\left(\chi_{\text {Be }}^{\text {slow }}\right)}{\operatorname{Re}\left(\chi_{e}\right)}\right)=30
$$

where $\nu_{\text {slow (fast) }}$ is the normalized Landau damping for the slow(fast) mode. Hence, in addition to having a larger Landau damping, the slow mode, in comparison to the fast mode, needs more laser energy to be sustained at a given amplitude.

\subsection{Evidence of kinetic effects}

Figure 8.2 compares two measured spectra, one without the interaction beam and the other with an interaction beam intensity of $\mathrm{I}=5 \times 10^{15} \mathrm{~W} \mathrm{~cm}^{-2}$. The variation in the amplitude of the thermal peaks is a direct indication of a change in ion temperature. Figure 8.3 shows the ion temperature increase as a function of the SBS excited ion-acoustic wave amplitude. When the energy in the interaction beam is increased, the ion-wave amplitude increases and the ion temperature rises; we found no change in the electron temperature. The ion temperature was normalized to the electron temperature in Fig. 8.3 to remove variations in the plasma parameters due to the heater beam. The electron temperature varied by less than $10 \%$ for a given heater beam energy and was independent of the interaction beam intensity. This direct observation of an increase in ion temperature indicates that en- 


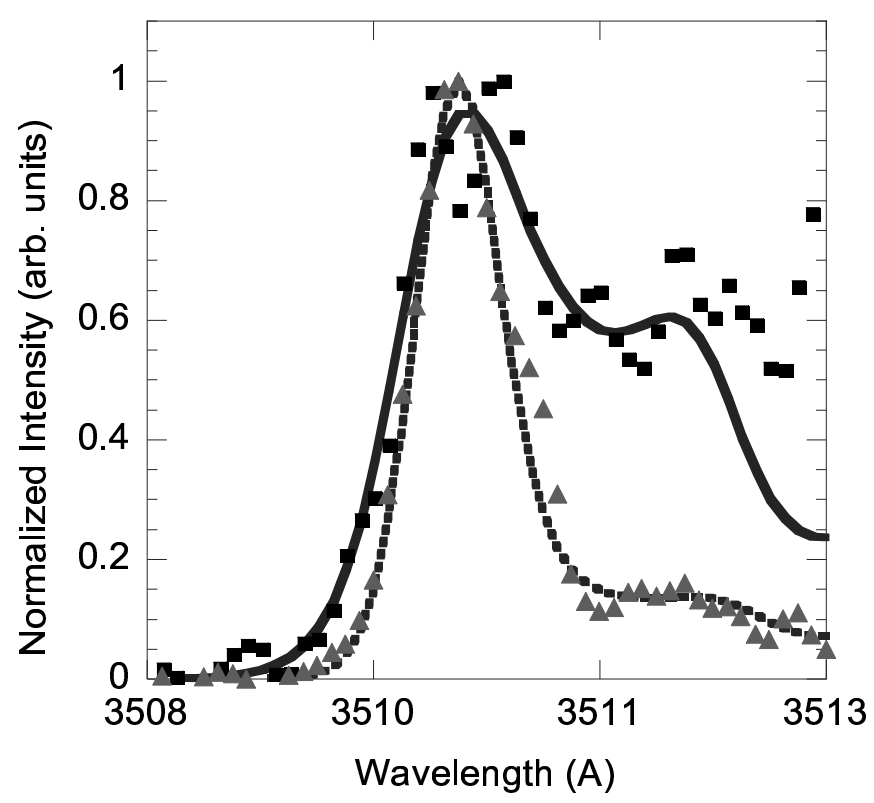

Figure 8.2: The blue-shifted (thermal) part of the Thomson-scattering spectra for a $10 \%$ $\mathrm{Au}$ mixture shows a shot without the interaction beam (triangles) and a shot with an interaction beam intensity of $6 \times 10^{15} \mathrm{~W} \mathrm{~cm}^{-2}$ (squares) resulting in an increase in ion temperature from 250 to $475 \mathrm{eV}$.

ergy is directly transferred from the SBS driven ion-acoustic waves into the ions. Trapping of Be ions is a plausible mechanism for such an energy transfer.

The SBS instability excites an ion-acoustic wave propagating in the same direction as the interaction beam; when this wave is driven to large amplitude it traps Be ions, accelerating them in its direction of propagation. After thermalization by collisions, the average ion temperature increases. We measure this increase in ion temperature from the light scattered off thermal ion-acoustic waves counter-propagating to the original hot ions.

Figure 8.4 shows the measured SBS reflectivity for the various mixtures of $\mathrm{Au}$ and Be. We observe the SBS reflectivity to be a few percent, while linear theory (Sec. 3.3) predicts a reflectivity of nearly $100 \%$. It is therefore clear that while our linear theory allows one to predict which ion-acoustic waves will be driven to large amplitudes by SBS [Fig. 8.1], 


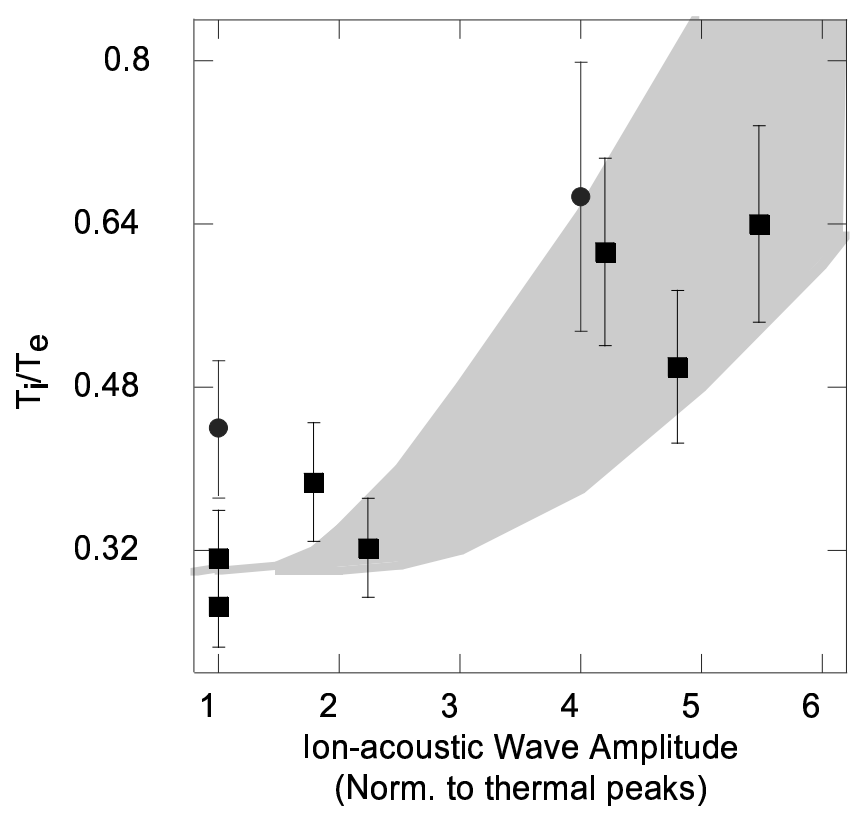

Figure 8.3: The ratio of $T_{i} / T_{e}$ as a function of the normalized ion-wave amplitude shows evidance of the generation of hot ions and ion trapping. The ion-wave amplitude is normalized to the thermal ion-wave amplitude. Data for Be plasmas with 5\% Au (circles) and 10\% $\mathrm{Au}$ (squares) show a factor of two increase in ion temperature. The gray area represents calculated results from a simple energy balance between energy deposition due to SBS, and a free-streaming heat flux. 


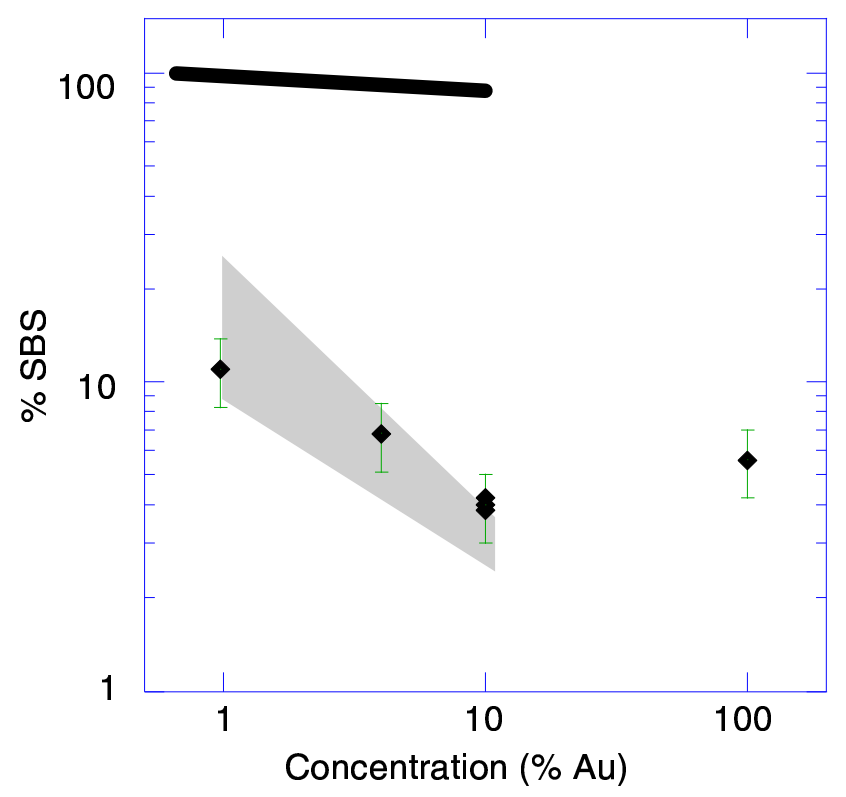

Figure 8.4: The SBS reflectivity has been measured to decrease for Be plasmas with increasing $\mathrm{Au}$ concentration (diamonds). This trend is reproduced by our non-linear SBS theory (shaded area). Linear calculations are also shown (black line).

the SBS reflectivity data (Fig. 8.4) indicates a need for a non-linear model that includes saturation of ion-acoustic waves. It should be noted that even after the ion temperature has increased, the fast mode is still weakly damped (see Fig. 2.2) and linear reflectivity predictions are close to $100 \%$, so in these experiments, saturation of the SBS can not be explained by an increase in Landau damping due to global heating of the ions [43].

We have applied the model developed in Sec. 3.4 where a non-linear frequency shift due to trapping detunes the resonant coupling between the interaction beam and the ion wave, therefore, saturating the instability. As the ion-acoustic waves are excited to large amplitude, ions are accelerated in the wave's potential wells, therefore, slowing the wave's phase velocity and detuning the SBS resonance so that energy is no longer coupled into these ion-acoustic waves by the incident laser. 
The SBS reflectivity for different fractions of Au is shown in Fig. 8.4 and is in rough agreement with the experimental values. Parameters used in the model were selected to match the experiment $\left(\mathrm{L}=800 \mu \mathrm{m}, \mathrm{T}_{i} / T_{e}=1 / 2, \mathrm{I}_{o}=3 \times 10^{15} \mathrm{~W}-\mathrm{cm}^{-2}, \mathrm{n}_{e}=10^{20} \mathrm{~cm}^{-3}\right)$. This model is dependent on the electron density and the interaction beam intensity; therefore, the shaded area in Fig. 8.4 result from uncertainties in these experimental parameters.

We further test this model by estimating the ion temperature in the Thomsonscattering volume. We balance the energy flux deposited into the acoustic waves (from the Manley-Rowe relations) with a free streaming heat flux [44]:

$$
\frac{\omega_{s}}{\omega_{0}}\left(R_{S B S} I_{0}\right)(z=L / 2)=n_{B e} T_{i}(z=L / 2) v_{B e}(z=L / 2) .
$$

This model assumes that the energy deposition is local, i.e., the mean-free path of the hot ions accelerated by trapping (a few tens of $\mu m$ ) is much smaller than the length of the plasma $(L \approx 1 \mathrm{~mm}$ ), and thermalization is fast enough so heat is carried away by Maxwellian ions when a steady-state is reached. Theoretical and experimental ion-wave amplitudes were related by matching a high reflectivity point; the model then reproduces the experimental scaling for ion temperature. This agreement shows that our modeling of SBS gives a correct estimation of the acoustic wave amplitude in the Thomson-scattering volume, but does not prove that trapping actually occurs; but trapping is the most plausible means for energy transfer form the waves to the ions with these plasma conditions. The fact that the electron temperature does not increase rules out inverse bremsstrahlung and other energy transfer through electrons.

Validation of the main assumptions of this modeling (a steady-state is reached and the non-linear frequency shift is the main effect that detunes and saturates the instability) 

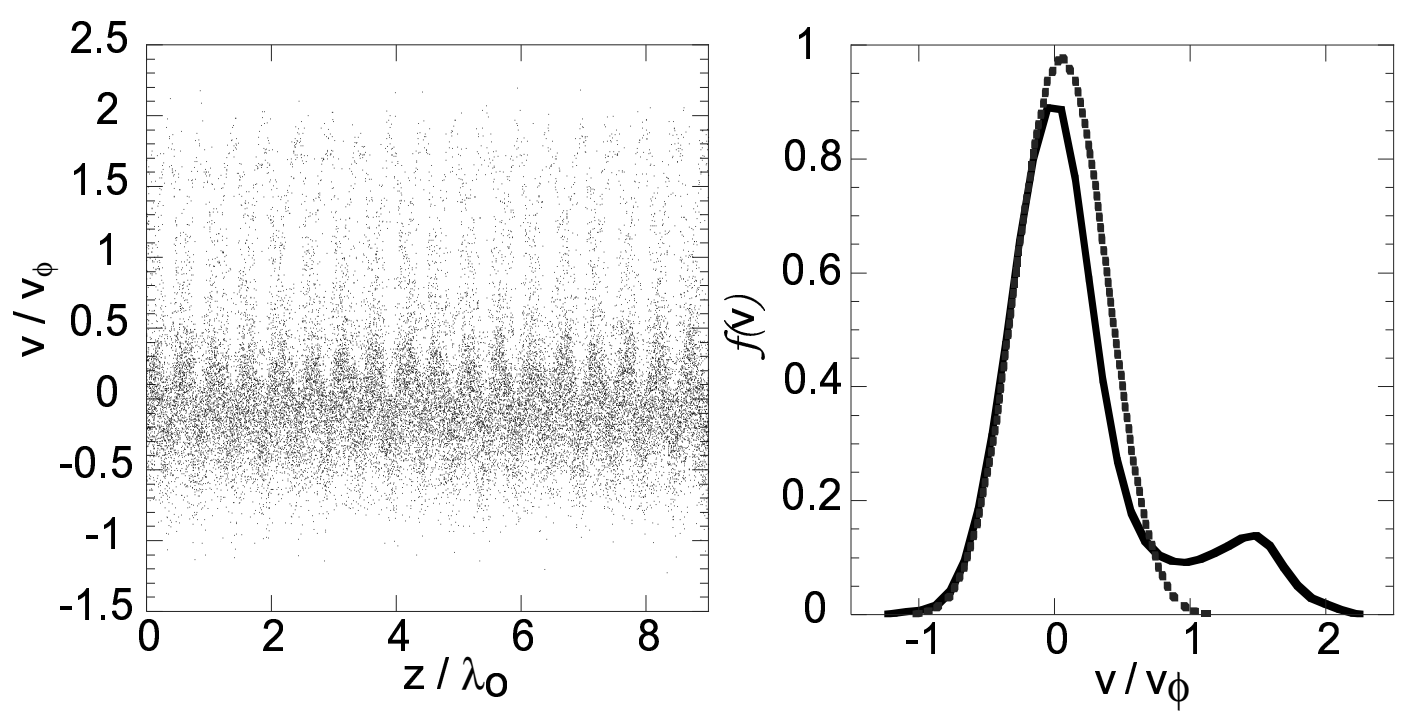

Figure 8.5: (a) The results of a PIC sumulation of SBS show a phase diagram where trapping can clearly be seen to repeat at the spatial frequency of the driven ion-acoustic wave. (b) The Be distribution function evolves from an intial Maxwellian (dashed line). After $60 \mathrm{ps}$ (solid line), a plateau at the phase velocity and a tail of hot ions is seen.

through comparisons of PIC and fluid simulations is beyond the scope of this thesis, but such a behavior has been emphasized in various publications $[4,45,3,46]$ and preliminary PIC simulations of SBS, with the experimental parameters, using the hybrid code Bzohar [7] show trapping of the Be ions by the SBS acoustic wave. Figure 8.5 shows the distribution of particles in phase space where trapped particles follow closed orbits and concurrently the distribution function exhibits a hot tail with ions moving up to twice the phase velocity.

\subsection{Summary}

In summary, we have presented measurements of the growth of ion-acoustic waves in well-characterized multi-ion-species plasmas. Our measurements of the stimulated Brillouin scattering (SBS) reflectivity indicate the need for a saturation mechanism. Using 
Thomson scattering and adding a small fraction of $\mathrm{Au}$ ions to a Be plasma enabled an accurate measurement of $T_{i}$; while $T_{i}$ was found to increase with SBS reflectivity and with the amplitude of the (local) acoustic waves, $T_{e}$ was found to be constant. This is consistent with our model where the increase in $T_{i}$ is due to trapping of ions by the SBS ion-acoustic waves, while the electron-ion collision rate and the electronic part of the Landau damping is very small, so that $T_{e}$ and $T_{i}$ are de-coupled. 


\section{Part IV}

\section{Summary and conclusions}




\section{Chapter 9}

\section{Summary}

\subsection{Introduction}

An understanding of the propagation of laser light into an underdense plasma is critical in the development of indirect-drive inertial confinement fusion. Understanding ionwave growth and the saturation mechanisms in these plasmas is necessary to improve the energy coupling into the fusion capsule. Currently models are being developed to predict stimulated Brillouin scattering (SBS) laser losses in high temperature, large scale length plasmas that will be achieved in inertial confinement fusion at future facilities such as the National Ignition Facility [40, 47]. We will present measurements that have verified both hydrodynamic (velocity gradients) and kinetic (trapping) effects on SBS.

The SBS instability results from the resonant coupling of an intense laser pulse, a scattered light wave, and an ion-acoustic wave. SBS excites an ion-acoustic wave from thermal fluctuations to large amplitude where it has been shown to saturate $[3,5,4,8]$. Previous models have suggested non-linear saturation mechanisms such as two-ion-decay, 
trapping, and wave breaking $[48,44,49,50]$. Theory has long predicted that velocity gradients will create a mismatch in the parametric SBS instability therefore, reducing the growth of ion-acoustic waves [37]. Detuning of SBS can be a powerful mechanism for limiting the growth of ion-acoustic waves because the instability relies on a resonant threewave process. Any shift in the local frequency of the ion-acoustic resonance relative to the ponderomotive frequency, created by the incident and reflected light, will limit the growth of the SBS instability.

Experiments at the Trident Laser Facility (see Sec. 5.1) observe detuning of the SBS instability by a velocity gradient and by kinetic effects. Direct evidence of detuning of SBS by a velocity gradient was observed through a novel use of two Thomson-scattering diagnostics allowing us to measure the frequency and amplitude of the ion-acoustic waves directly responsible for SBS. By comparing the local ion-acoustic frequency, with the local frequency of the driven acoustic wave we have measured the actual detuning of the SBS instability.

We will show that ion-acoustic waves and the SBS reflectivity saturate for intensities above I $>1.5 \times 10^{15} \mathrm{~W} \mathrm{~cm}^{-2}$ where we have seen direct effects of trapping. Adding a small amount of $\mathrm{Au}$ to our Be plasmas allowed the accurate determination of the ion temperature by measuring the relative damping of two ion-acoustic modes. We report up to a factor of two increase in ion temperature when ion-acoustic waves are excited to large amplitude by SBS [39]. This increase in ion temperature is a strong indication of hot ions due to trapping. 


\subsubsection{Thomson scattering}

Thomson scattering provides a measure of the frequencies and amplitudes of coand count-propagating ion-acoustic waves in a plasma. From the measured resonant frequencies, the local sound speed, electron temperature, and particle flow can be determined. The amplitude of the ion-acoustic wave is proportional to the square root of the measured intensity. In multi-ion-species plasmas, the relative amplitude of the light scattered from ion-acoustic waves allows an accurate determination of the ion temperature (see Sec. 4.3). In theory, the ion temperature can also be obtained from the width of the intensity peaks in the Thomson-scattering spectra, but velocity and temperature gradients within the Thomson-scattering volume make this measurement uncertain and therefore, unreliable in laser produced plasmas.

In our experiment, the wavelength of the probe laser, the scattering geometry, and the plasma parameters result in collective Thomson scattering from fluctuations characterized by wave numbers such that the scattering parameter is greater than one, $\alpha=1 / \mathrm{k}_{a} \lambda_{D} \approx$ 3 , where $\mathrm{k}_{a}$ is the acoustic scattering wave vector and $\lambda_{D}$ is the electron Debye length.

When observing low frequency collective effects with Thomson scattering, the frequency of the scattered light is shifted from the laser frequency in two ways: by the frequency of the ion-acoustic wave in the frame of the plasma $\left(\omega_{a}=\mathrm{c}_{s} k_{a}\right)$, and by the relative ion flow in the laboratory frame $\left(\mathrm{v}_{f}\right)$. The frequency shift due to the ion-acoustic waves is given by,

$$
\Delta \lambda_{\text {iaw }}=\frac{\lambda_{3 \omega}^{2}}{2 \pi c}\left(\mathrm{c}_{s} \mathrm{k}_{a}\right)
$$


while the ion flow shifts the spectral peaks by,

$$
\Delta \lambda_{\text {flow }}=\frac{\lambda_{3 \omega}^{2}}{2 \pi c}\left(\overrightarrow{\mathrm{k}}_{a} \cdot \overrightarrow{\mathrm{v}}_{f}\right)
$$

where the dispersion relation is given by,

$$
\frac{\omega}{k}=c_{s}=\sqrt{\frac{Z k_{b} T_{e}}{m_{i}\left(1+k^{2} \lambda_{D e}^{2}\right.}+3 \frac{k_{b} T_{i}}{m_{i}}} .
$$

$\mathrm{k}_{b}$ is Boltzmann's constant and $\mathrm{m}_{i}$ is the ion mass and $\mathrm{Z}$ is the particles average charge state. Therefore, if the sound speed is known, the frequency shift of one peak in the Thomsonscattering spectra relative to the frequency of the probe laser, provides a measure of the plasma flow.

We employed two separate Thomson-scattering spectrometers observing the same volume and location in the plasma (Fig. 7.1a); one of the Thomson-scattering diagnostics measured light scattered from the SBS excited ion-acoustic waves $\left(\mathbf{k}_{a}=\mathbf{k}_{\|}\right.$see Fig. $\left.7.1 \mathrm{~b}\right)$ which propagate parallel to the interaction beam (Fig. 9.1a). The other Thomson-scattering diagnostic probes ion-acoustic waves propagating perpendicular $\left(\mathrm{k}_{a}=\mathrm{k}_{\perp}\right.$ see Fig. $\left.7.1 \mathrm{~b}\right)$ to the interaction beam (Fig. 9.1b). The latter allows us to measure the local sound speed (proportional to the electron temperature), while the former measures both the frequency of the excited ion-acoustic wave (from $\mathbf{k}_{||}$co-propagating in the direction of the interaction beam) and the plasma flow along the interaction beam $\left(-\mathbf{k}_{||}\right.$counter-propagating in the direction of the interaction beam). 


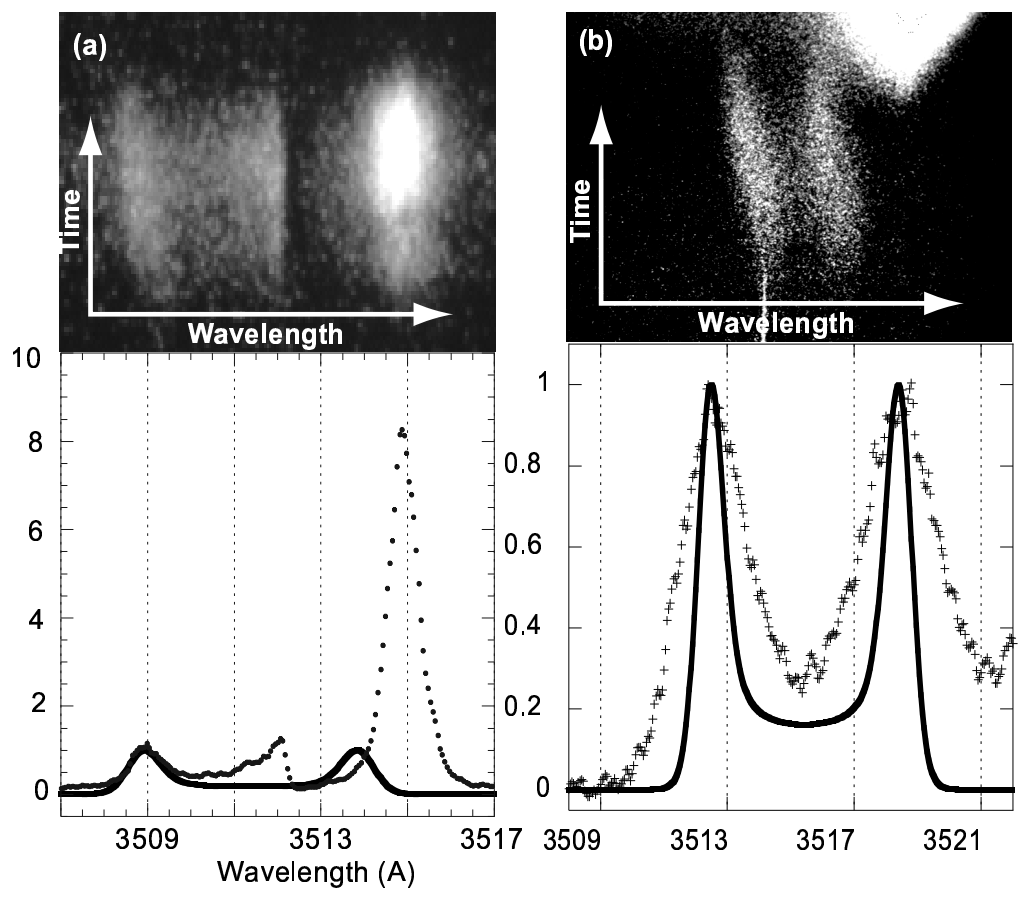

Figure 9.1: (a) The driven Thomson scattering spectra provides a measure of both the plasma flow along the axis of the interaction beam $\left(-\mathbf{k}_{\|}\right)$, the frequency of the SBS driven acoustic wave $\left(\mathbf{k}_{\|}\right)$, and the relative amplitude of the SBS driven ion-acoustic waves. This data is representative of a point at $\mathrm{z}=-375 \mu \mathrm{m}$ and $\mathrm{x}=400 \mu \mathrm{m}$ with an interaction beam intensity of $\mathrm{I}=1.5 \times 10^{15} \mathrm{~W} \mathrm{~cm}^{-2}$. (b) Our thermal Thomson-scattering spectra allow us to measure the electron temperature by observing ion-acoustic waves propagating perpendicular $\left(\mathrm{k}_{\perp}\right)$ to the driven ion-acoustic waves $\left(\mathrm{k}_{\|}\right)$. 


\subsection{Plasma characterization}

\subsubsection{Plasma temperature}

The plasma has been well-characterized (see Chapt. 6); Figure 9.2 shows a constant electron temperature profile $\left(\mathrm{T}_{e}(\mathrm{x}=400 \mu \mathrm{m})=450 \mathrm{eV}\right)$ along the interaction beam axis. Figure $9.1 \mathrm{~b}$ shows a typical Thomson-scattering spectrum $\left(\mathrm{k}_{\perp}\right)$. Two peaks corresponding to thermal ion-acoustic fluctuations propagating perpendicular to the interaction beam $\left(\mathrm{k}_{\perp}\right)$ are evident. The data are fit using a standard theoretical form factor which uses the electron temperature as the only sensitive free parameter for our fully ionized Be plasmas (see Sec. 4.2). The measured width of the peaks $(\delta \lambda)$ gives an estimated velocity range $\frac{\delta v}{c_{s}}\left(\propto \frac{\delta \lambda}{\lambda}\right)=0.5$ within the Thomson-scattering volume. This broadening is to be expected as the initial plasma length is only $\mathrm{y}=100 \mu \mathrm{m}$ in the perpendicular direction and therefore, the Thomson-scattering volume is centered around the outward expansion of the plasma. An error of $20 \%$ in the electron temperature is due to the broad Thomson-scattering spectra. The error bars were determined by fitting the form factor over a reasonable spread in the data.

The ion temperature has been measured using Be plasmas with small amounts of Au. Measuring the relative damping of two kinetic modes produced in these multi-ionspecies plasmas allowed us to determine the ion temperature with high accuracy. In this study we found the ion temperature to be $\mathrm{T}_{i}(\mathrm{x}=300 \mu \mathrm{m})=250 \mathrm{eV}$ (see Sec. 4.3). 


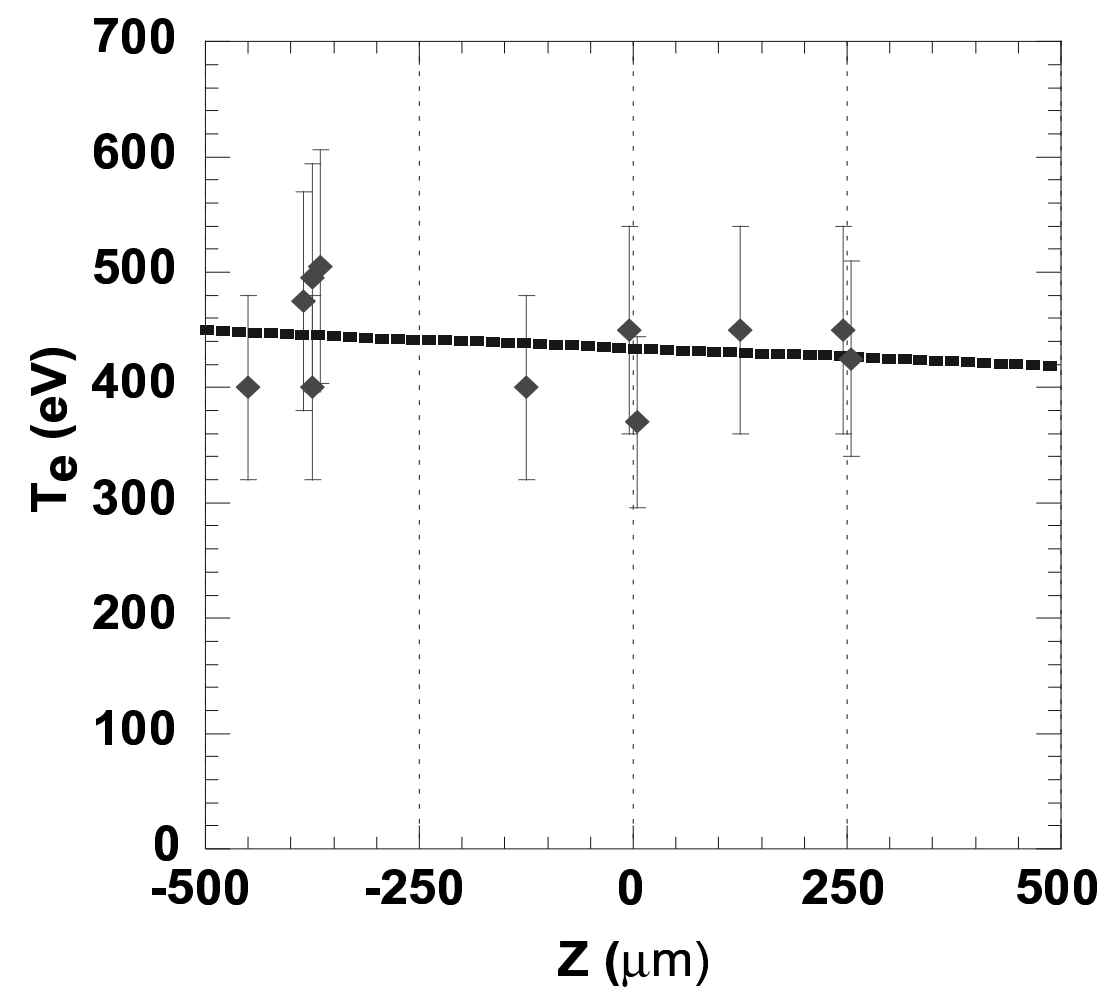

Figure 9.2: The sound speed (right axis) and therefore the electron temperature (left axis) were measured to be a constant $\mathrm{T}_{e}=450 \mathrm{eV}$ along the interaction beam axis, $\mathrm{x}=400 \mu \mathrm{m}$ from the surface of the target. The line is a best fit. 


\subsubsection{Plasma flow}

Figure 9.1a shows a Thomson-scattering spectra in which the probed ion-acoustic waves are co- and counter-propagating in the direction of the interaction beam $\left(\mathrm{k}_{\|}\right)$. The blue-shifted light is scattered from ion-acoustic fluctuations $\left(-\mathbf{k}_{\|}\right)$, i.e., the standard Thomsonscattered signal. The form factor is fit to the blue-shifted intensity peak using the independently measured electron temperature and a flow of $\frac{\mathrm{v}}{c_{s}}=0.7$. The red-shifted intensity peak in the theoretical fit $(\lambda=3513.8 \AA)$ corresponds to the local ion-acoustic resonance for waves co-propagating in the direction of the interaction beam $\left(\mathbf{k}_{\|}\right)$. Note that the use of two Thomson-scattering diagnostics is necessary as the driven acoustic wave is not assumed to be at the local resonant frequency. In fact, from Fig. 9.1a it is evident that the SBS driven ion-acoustic waves are off resonance (see Sec. 9.3.1).

By moving the target, we have used scattering from the ion-acoustic fluctuations $\left(-\mathbf{k}_{||}\right)$to map out the velocity flow (Fig. 9.3) (see Sec. 6.6). A $200 \mu \mathrm{m}$ velocity plateau in the middle of the plasma is evident while a Mach 1 flow was measured in the front of the plasma. A LASNEX [29] simulation using a 1-mm circular target with an average intensity of $10^{14} \mathrm{~W} \mathrm{~cm}^{-2}$ calculated a radial flow $400 \mu \mathrm{m}$ from the target that compares well with our data in Fig. 9.3.

\subsubsection{Plasma electron density}

The frequency of the light backscattered by the electron plasma wave (SRS) is a strong function of the electron plasma frequency $\left(\omega_{p e}^{2}=\frac{n_{e} e^{2}}{\varepsilon_{o} m_{e}}\right)$ and therefore, the density (see Sec. 6.2), 


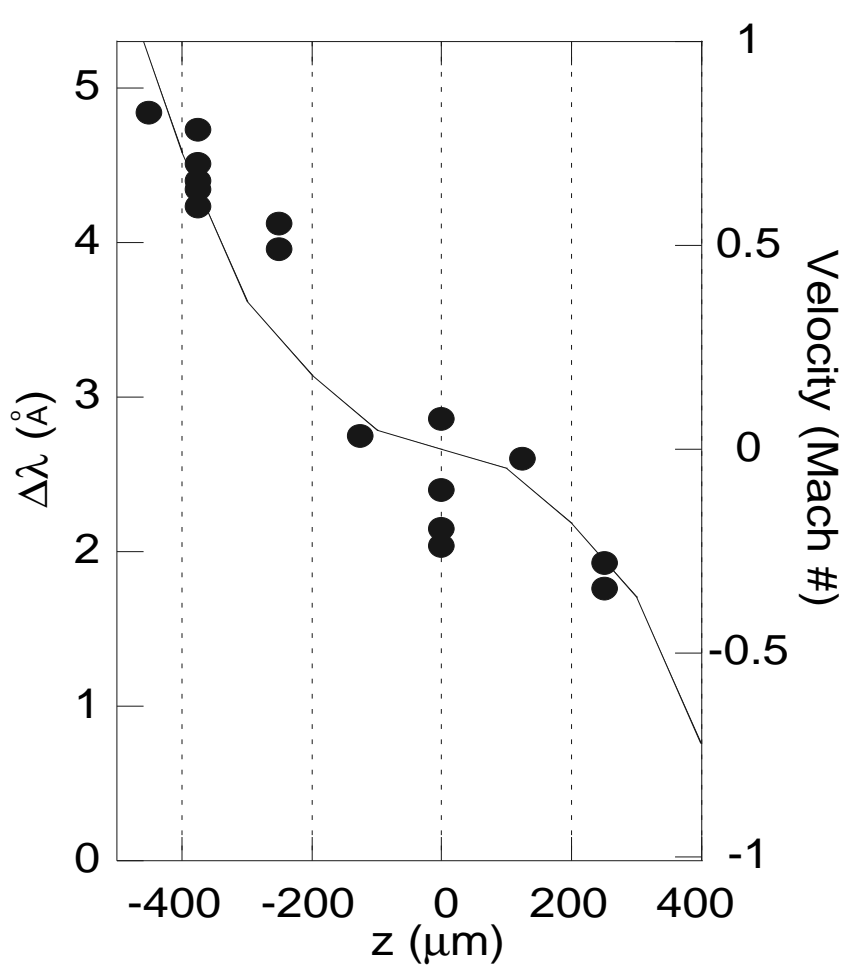

Figure 9.3: The frequency shift of the peak intensity scattered from thermal $\left(-\mathbf{k}_{\|}\right)$ionacoustic waves allows us to measure a velocity profile along the interaction beam axis. A LASNEX simulation using a 1-mm disk target reproduces the velcocity profile. 

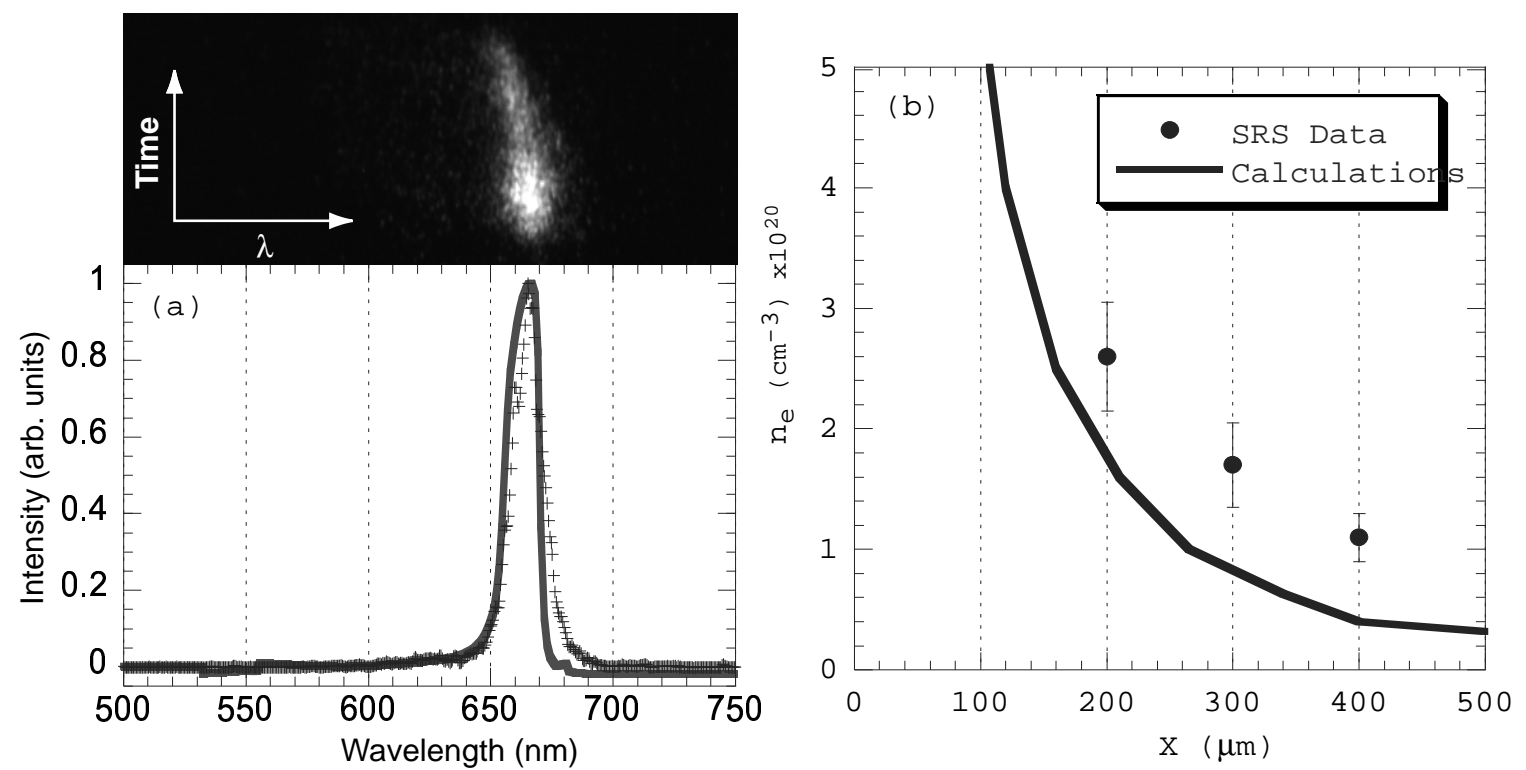

Figure 9.4: (a) The SRS spectra is measured at $\mathrm{x}=400 \mu \mathrm{m}$ from the target surface. A normalized linear gain calculation is fit to the data (line) to obtain the density. (b) The electron density calculated from the SRS spectra are plotted as a function of the distance from the target surface. LASNEX calculations are plotted (line).

$$
\omega=\sqrt{\omega_{p e}^{2}+\frac{3 \mathrm{k}^{2} k_{b} \mathrm{~T}_{e}}{\mathrm{~m}_{e}}}
$$

where $\mathrm{m}_{e}$ is the mass of an electron, and $\mathrm{k}=2 k_{o}$ is the wave-vector of the probed electron plasma wave.

Figure $9.4 \mathrm{a}$ shows the measured SRS spectrum for a shot where the interaction beam was focused $\mathrm{x}=400 \mu \mathrm{m}$ from the target surface. The spectrum peaks at $665 \mathrm{~nm}$ which corresponds to a density of $\mathrm{n}_{e}(\mathrm{x}=400 \mu \mathrm{m})=1.2 \times 10^{20} \mathrm{~cm}^{-3}$. The spectra was fit using a linear gain calculation. To match the spectral width a $10 \%$ density gradient was assumed over $300 \mu \mathrm{m}$. The corresponding SRS gain was $\mathrm{G}_{\text {srs }}=10$. The electron density profile normal to the target surface is shown in Fig. 9.4b. 


\subsection{Saturated SBS regime}

Figure 9.1a shows Thomson-scattering data in which the SBS instability driven by the interaction beam, has excited ion-acoustic waves $\left(\mathbf{k}_{\|}\right)$to large amplitude. The intensity of the scattered light is proportional to the square root of the amplitude of the probed ion-acoustic wave $\left(\mathrm{I}^{2} \propto \frac{\delta n}{n_{e}}\right)$. In Fig. 9.5, the local normalized ion-acoustic wave amplitude $(\mathrm{z}=-375)$ and the SBS reflectivity are plotted as a function of the intensity of the interaction beam. The amplitude of the local SBS driven ion-acoustic waves and the SBS reflectivity saturate for intensity $\mathrm{I}>1.5 \times 10^{15} \mathrm{~W} \mathrm{~cm}^{-2}$. The intensity was varied by changing the energy from 10 to $150 \mathrm{~J}$ while all other parameters were held fixed.

Various saturation mechanisms have been suggested to explain ion-wave saturation, including frequency detuning induced by trapping [3, 9, 39], 2-ion-wave-decay [8, 51, 7, 52], increased linear Landau damping due to kinetic ion heating [53, 43], and nonlinear damping associated with wave-breaking [48, 44], and energy coupling with higher harmonics $[54,55]$. Detuning of SBS can be a powerful mechanism for limiting the growth of ion-acoustic waves because the instability relies on a resonant three-wave process. Any shift in the local frequency of the SBS driven ion-acoustic wave relative to the frequencies of the incident and/or reflected light will limit the growth of the SBS instability. In the following sections we will investigate detuning of the local ion-acoustic wave due to velocity gradients and kinetic effects. We have found that velocity gradients increase the threshold for linear growth, but for intensities above $\mathrm{I} \geq 1.5 \times 10^{15} \mathrm{~W} \mathrm{~cm}^{-2}$ non-linear kinetic effects saturate SBS. 


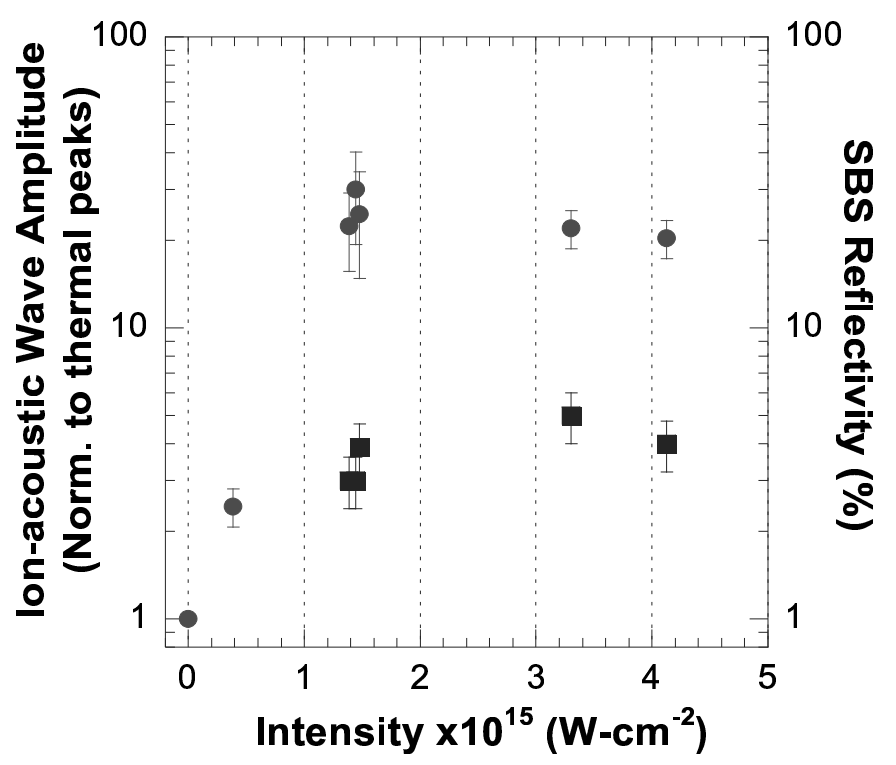

Figure 9.5: The ion-acoustic wave amplitude and the SBS reflectivity are plotted as a function of the intensity of the interaction beam. The target was moved to probe ion waves at $z=-375 \mu \mathrm{m}$. The plasma length along the interaction beam was $\mathrm{L}=1-\mathrm{mm}$.

\subsubsection{Velocity gradient}

The measured velocity gradient Doppler shifts the local resonant frequency of the ion-acoustic waves, detuning the SBS resonance, therefore, saturating the driven ionacoustic wave. From Fig. 9.1a it is evident that the SBS driven ion-acoustic waves are are off resonance. The local resonant frequency for acoustic waves with $\mathbf{k}_{\|}$(shown by the red-shifted intensity peak in the theoretical form factor) is lower than that of the measured SBS driven ion-acoustic wave by $1.1 \AA$, which proves that SBS is detuned by the velocity gradient (i.e. SBS-driven acoustic waves are locally off resonance). Figure 9.6 shows that the frequency of the driven ion-acoustic wave remains unchanged as one moves away from the velocity plateau and towards the front of the plasma. This indicates that the frequency of the ponderomotive force driving the ion-acoustic waves in the front of the plasma is 
constant.

From Fig. 7.4 it is apparent that SBS grows rapidly in the plateau creating a large scattered light wave. This scattered light wave beats with the incident light wave creating a constant frequency ponderomotive force in the front of the plasma (i.e., the frequency of the light scattered from the plateau and the frequency of the incident light are constant). From the resonant acoustic wave, it is apparent that the acoustic resonance is changing as one move towards the front of the plasma, therefore, the constant ponderomotive frequency created in the plateau is increasingly off resonance in the front of the plasma and the spatial amplitude of the ion-acoustic waves can not resonantly grow in the front of the plasma.

The experiment was simulated in 2-D using pF3d [40]. A $f / 6$ RPP beam with a best focus of $60 \mu \mathrm{m}$ and an average vacuum intensity of $10^{15} \mathrm{~W} \mathrm{~cm}^{-2}$ was propagated through a $\mathrm{L}=1000 \mu \mathrm{m}$ slab of plasma $\left(\mathrm{T}_{e}=450 \mathrm{eV}, \mathrm{n}_{e}=1.2 \times 10^{20} \mathrm{~cm}^{-3}\right)$. A Mach 2 transverse flow and the measured velocity profile along the propagation axis were included (right axis Fig. 9.6)

Figure 9.6 shows the simulated spatial growth of the ion-acoustic waves. The calculated amplitude profile is shown in Fig. 7.4 after post processing the simulation and averaging over a $70 \mu \mathrm{m}$ area similar to the experiments. It is evident that large ion-acoustic waves are driven only in the front half of the plasma. The velocity gradient in the back of the plasma prevents any significant SBS growth. The plateau in the middle provides a resonant region where SBS drives a large ion-acoustic wave. In the front of the plasma, the strong velocity gradient prevents SBS growth at the local resonant frequency, but the off resonance ponderomotive force drives non-resonant acoustic waves in the front of the 


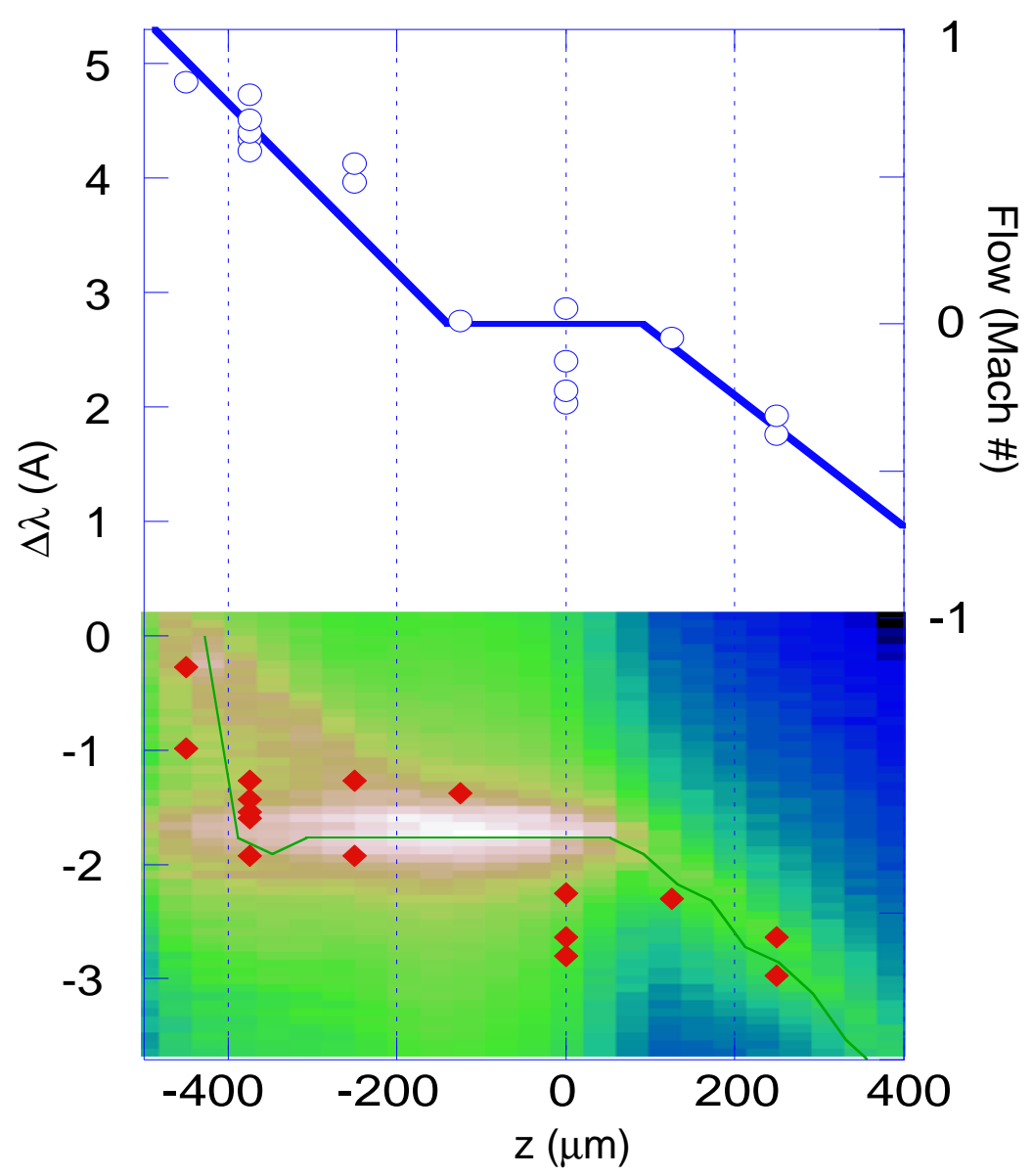

Figure 9.6: (color). The wavelength shift of the peak intensity for the down shifted (solid squares) and up-shifted (open circles) Thomson-scattering peaks are plotted. The up-shifted intensity peaks reveal a Mach 1 flow parallel to the interaction beam (right axis). The data are plotted on top of a pF3d simulation where ion-acoustic waves are driven to large amplitude in the front of the velocity plateau. 
plasma (Fig. 9.6).

While the amplitude of the driven acoustic waves $\left(\frac{\delta n}{n_{e}}\right)$ is readily obtained from simulations, it has to be calculated from the measured intensity peaks in the Thomsonscattering spectra. This is done by comparing the scattered reflectivity from thermal fluctuations (i.e. the power scattered in to the left intensity peak in Fig. 9.1a divided by the incident laser power), which is given by:

$$
R_{\text {thermal }}=\frac{1}{4} r_{0}^{2} n_{e} L_{3 \omega} \Delta \Omega_{e x p}
$$

where $r_{0}$ is the classical electron radius, $L_{3 \omega}$ is the length along the direction of the $3 \omega$ probe beam, and $\Delta \Omega_{e x p}$ is the solid angle of the collecting optics (an f/5 lens), with the reflectivity scattered by the driven acoustic wave given by:

$$
R_{\text {driven }}=\frac{1}{4} r_{0}^{2} n_{e}^{2} \lambda_{3 \omega}^{2} L_{3 \omega} L_{c}\left(\frac{\delta n}{n_{e}}\right)^{2}
$$

where $\delta n / n_{e}$ is the amplitude of the acoustic wave and $L_{c}$ its correlation length along the direction of the $3 \omega$ probe beam. $L_{c}$ can be estimated by the transverse size of speckles generated by the interaction beam $f_{2 \omega} \lambda_{2 \omega}$.

Figure 9.7 shows that this estimation of $\delta n / n_{e}$ reproduces the overall spatial shape predicted by pF3d, but the absolute amplitude is smaller by almost an order of magnitude. Various factors can explain this discrepancy. For instance, any misalignment of the optics, an overestimation of $L_{c}$, or enhanced thermal fluctuations would lead to an underestimation of the acoustic wave amplitude. Assuming the latter, we have verified that increasing the amplitude of the thermal noise source used both in the Thomson-scattering calculations and in the SBS simulations by an order of magnitude gives a good agreement between the 


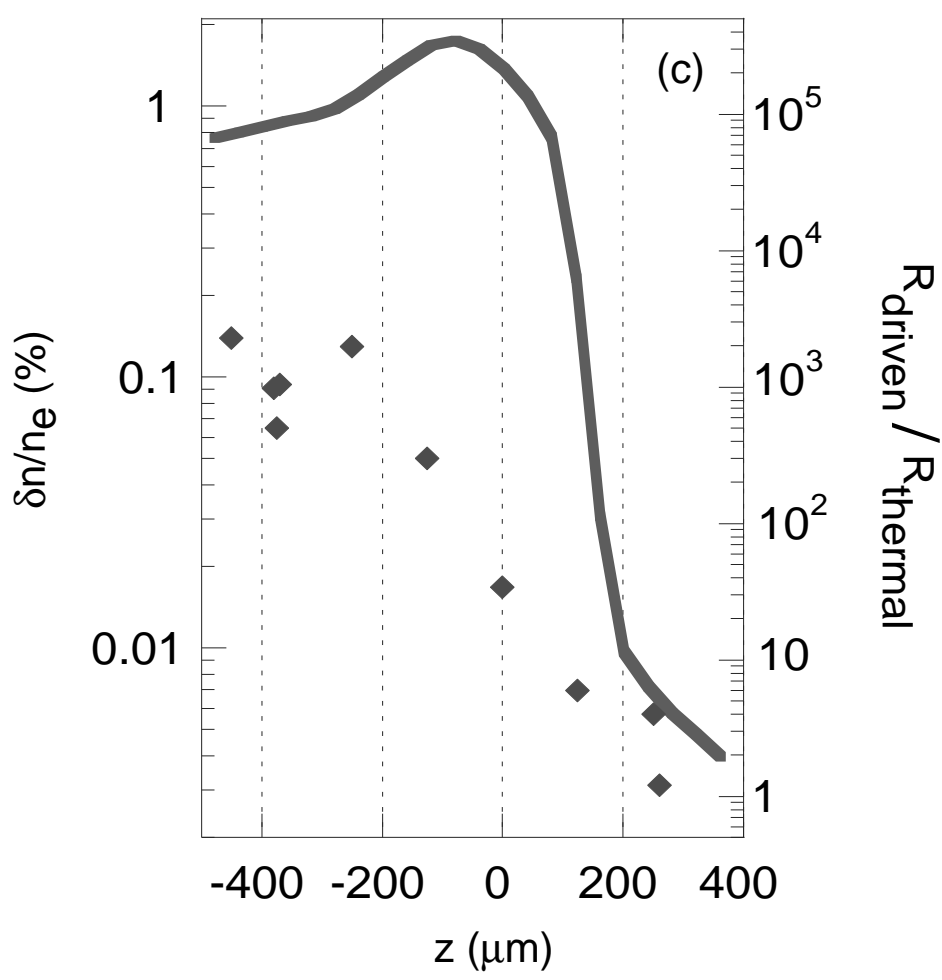

Figure 9.7: The measured amplitude of the excited ion-acoustic wave $\left(\frac{\delta n}{n_{e}}\right)$ is plotted as a function of the position along the interaction beam. The calculated amplitude profile from pF3d (solid line) after averaging over a $70 \mu \mathrm{m}$ area similar to the experiment is shown.

measured and simulated wave amplitude and spatial profiles. A study of the amplitude of the thermal noise in laser induced plasmas needs to be completed to investigate the discrepancies in the calculated wave amplitudes seen in this and other studies, see Ref. [19].

Figure $9.8 \mathrm{a}$ plots the average SBS reflectivity as a function of the length of the plasma along the interaction beam (L). This scaling was done by using various sizes of targets. The SBS reflectivity should scale exponentially with the length of the velocity plateau. From simulations it is apparent that there is no velcocity plateau for targets shorter than 600 microns, therefore, the reflectivity below this point is due to scattering from regions of the plasma where there is a velocity gradient. In these cases, the reflectivity 

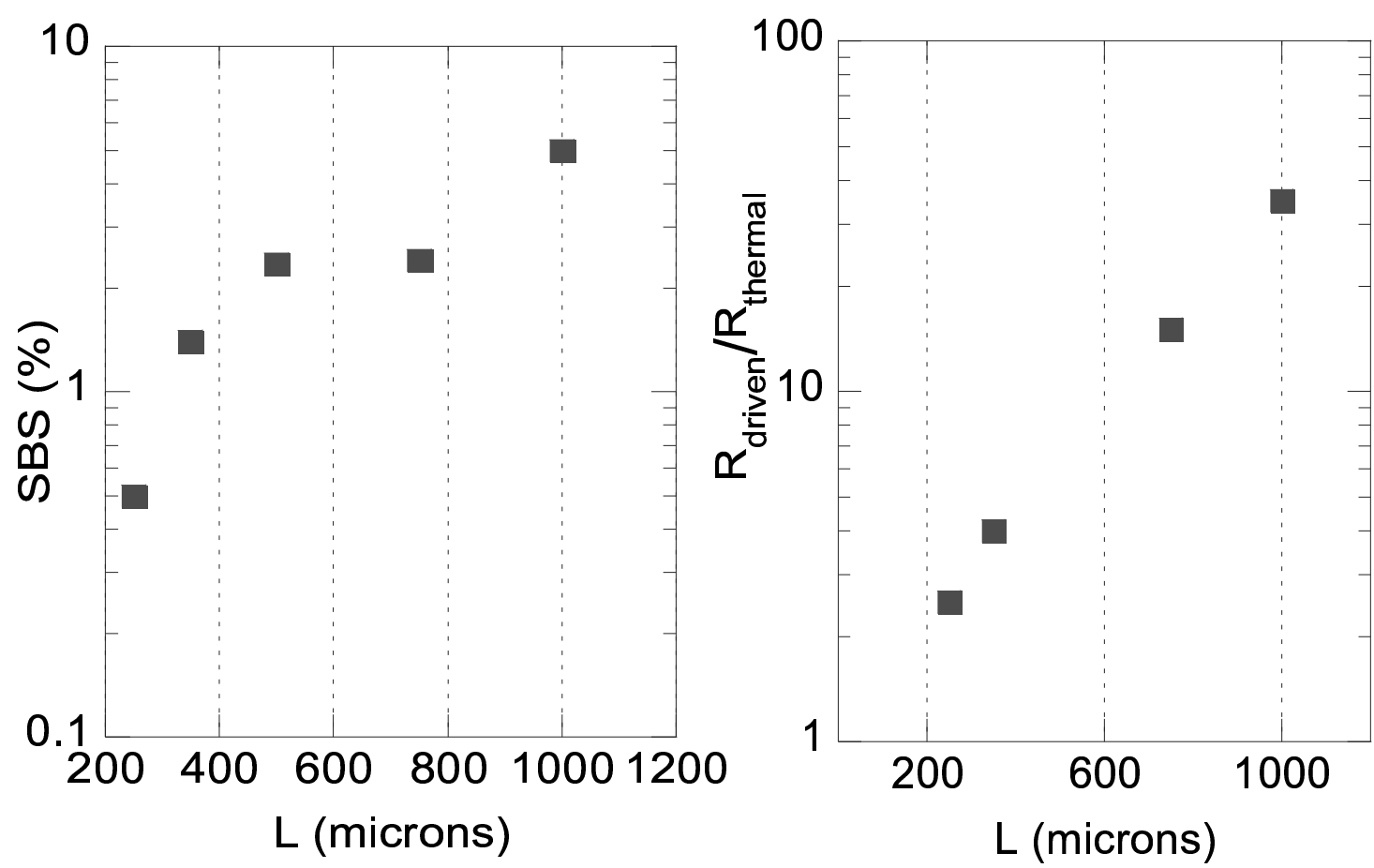

Figure 9.8: The (a) SBS reflectivity and (b) normalized ion-wave amplitude is plotted as a function of plasma length along the interaction beam axis.

is very sensitive to the actual shape of the velocity gradient and no simple scaling is available. As shown previously, a very precise characterization of the plasma conditions was necessary to model SBS from the largest 1-mm targets (see Sec. 9.3.1). Figure 9.8b shows the measured ion-acoustic wave ratio $\left(\mathrm{R}_{\text {driven }} / R_{\text {thermal }}\right)$ in the middle of the plasma $(\mathrm{z}=0)$ as a function of the plasma length along the interaction beam $(\mathrm{L})$.

\subsubsection{Kinetic effects (Trapping)}

When the intensity exceeds I $>1.5 \times 10^{15} \mathrm{~W} \mathrm{~cm}^{-2}$, it is clear that even with a strong velocity gradient, non-linear kinetic effects are needed to explain saturation of the SBS instability. In our range of experimental parameters $\left(6<Z_{B e} T_{e} / T_{i}<14\right)$ there are 
many particles at the ion-acoustic phase velocity, therefore non-linear kinetic effects are a plausible mechanism to saturate SBS.

By adding small amounts of $\mathrm{Au}$ to our Be plasmas, we were able to accurately measure an increase in ion temperature when ion-acoustic waves were driven to large amplitude. For these plasmas, the solution to the kinetic dispersion relation reveals two modes which react qualitatively differently when the ion temperature is changed. Namely, when the ion temperature is increases, the damping of the two modes move inversely. Figure 9.9a shows the multi-ion spectra for two different ion temperatures. These data are fit using a multi-ion form factor with two free sensitive fit parameters $\left(\mathrm{T}_{i}, \mathrm{~T}_{e}\right)$ [23]. The frequency shift of the outter peak is set by the electron temperature, while the relative amplitude of the two intensity peaks is determined by the ion temperature.

Figure $9.9 \mathrm{~b}$ shows the ion temperature increase as a function of the SBS excited ion-acoustic wave amplitude. As the interaction beam excites ion-acoustic waves, the ion temperature in the plasma was measured to increase, while we found no change in the electron temperature. Comparing Fig. 9.5 and Fig. 9.9b it is evident that the increase in ion temperature is directly linked to the saturated regime. This direct observation of an increase in ion temperature is an indication of ion trapping. In this experiment ion-acoustic waves are excited in the direction of the interaction beam. These driven acoustic waves trap ions which are then accelerated creating hot ions. After thermalization this will increase the average ion temperature which we have measured using Thomson scattering.

Trapping induces a modification of the dispersion relation for the SBS acoustic wave, creating a non-linear frequency shift $[7,15,9]$. This non-linear frequency shift detunes 


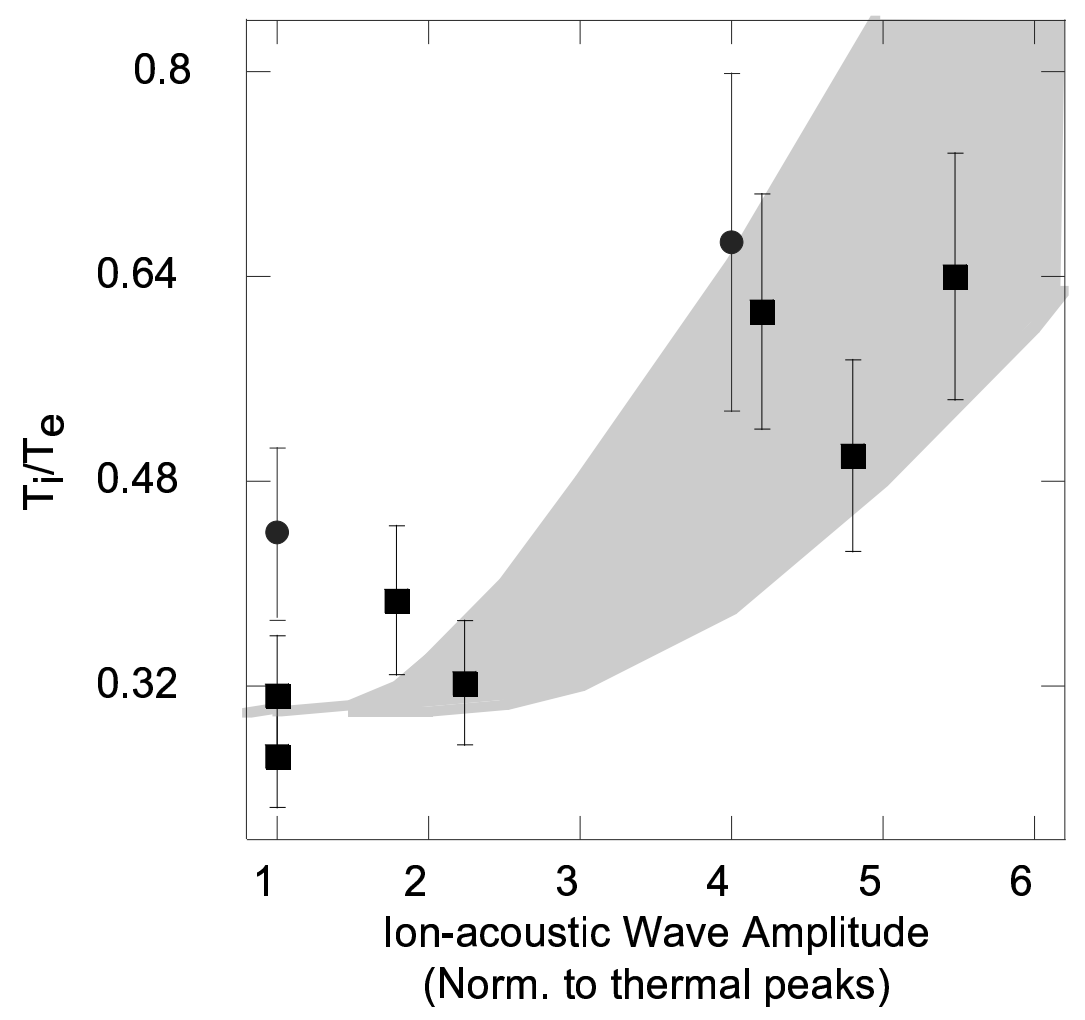

Figure 9.9: The ratio of $\mathrm{T}_{e} / T_{i}$ as a function of the normalized ion-wave amplitude shows evidence of the generation of hot ions and ion trapping. The ion-wave amplitude is normalized to the thermal ion-wave amplitude. Data for Be plasmas with 5\% Au (circles) and $10 \%$ $\mathrm{Au}$ (squares) show a factor of two increase in ion temperature. The gray area represents calculated results from a simple energy balance between energy deposition due to SBS, and a free-streaming heat flux. 
the SBS resonance therefore, saturating the ion-acoustic wave. Using this trapping model, the calculated SBS reflectivity is close to the $5 \%$ measured in our experiments [38].

Furthermore, we have tested this model by estimating the ion temperature in the Thomson-scattering volume. We balance the energy flux deposited into the ion-acoustic waves (from the Manley-Rowe relations) with a free streaming heat flux [44]. This model assumes that the energy deposition is local and thermalization is fast enough so that heat is carried away by Maxwellian ions when a steady state is reached. Theoretical and experimental ion-wave amplitudes were related by matching a high reflectivity point; the model then reproduces the experimental scaling for ion temperature (Fig. 9.9b).

\subsection{Summary}

We have presented a detailed study of SBS saturation mechanisms in low-Z Be plasmas. We find that detuning of the SBS instability by velocity gradients and by trapping are effective mechanisms for saturating the SBS reflectivity. Direct evidence of detuning of SBS by a velocity gradient was observed through a novel use of two Thomson-scattering diagnostics, allowing us to directly measure the frequency shift between the frequency of the SBS driven acoustic wave relative to the local resonant acoustic frequency. Furthermore, we have shown that by adding small amounts of $\mathrm{Au}$ to our Be plasmas have allowed us to accurately determine the ion temperature by measuring the relative damping of two ion-acoustic modes. We report up to a factor of two increase in ion temperature when ionacoustic waves are excited by SBS. This increase in ion temperature is a strong indication of hot ions due to trapping. 
Trapping is an efficient saturation mechanism in low-Z plasmas because there are significant particles at the wave's phase velocity, but in general trapping does not explain saturation in high-Z plasmas. Both the ion-acoustic wave and SBS reflectivity have experimentally been shown to saturate in high-Z plasmas [19]. Previous non-linear saturation mechanisms such as two-ion-decay or harmonic generation in high-Z plasmas have been suggested, but these effects have never been experimentally or theoretically verified.

In part due to the measured kinetic effects, trapping is currently being implemented in models to help simulate low-Z conditions [56], and experiments are underway to investigate saturation mechanisms in high-Z plasmas. Understanding the saturation mechanisms in high-Z plasmas is critical in the development of indirect drive inertial confinement fusion. 


\section{Chapter 10}

\section{Conclusions}

We have shown that detuning of the SBS instability by velocity gradients and trapping are effective mechanisms in Low-Z Be plasmas for saturating the SBS reflectivity. Direct evidence of detuning of SBS by a velocity gradient was observed through a novel use of two Thomson-scattering diagnostics, allowing us to directly measure the frequency shift of the SBS driven acoustic wave relative to the local acoustic frequency. Furthermore, experiments using multi-ion-species plasmas have allowed us to accurately determine the ion temperature by measuring the relative damping of two ion-acoustic modes. We report upto a factor of two increase in ion temperature when ion-acoustic waves are excited by SBS. This increase in ion temperature is a strong indication of hot ions due to trapping.

Trapping is an efficient saturation mechanism in low-Z plasmas because there are significant particle at the wave's phase velocity, but in general trapping does not explain saturation in high-Z plasmas. Both the ion-acoustic wave and SBS reflectivity have experimentally been shown to saturate [19]. Previous theories have suggested non-linear 
saturation mechanisms such as two-ion-decay or harmonic generation in high-Z plasmas, but these effects have never been experimentally or theoretically verified.

In part due to the measured kinetic effects, trapping is currently being implemented in models to help simulate low-Z conditions and experiments are underway to investigate saturation mechanisms in high-Z plasmas. Understanding the saturation mechanisms in high-Z plasmas is critical in the development of indirect drive inertial confinement fusion. 


\section{Bibliography}

[1] Dwight R. Nicholson. Introduction to Plasma Theory. Wiley, 1983.

[2] F. F. Chen. Introduction to plasma physics and controlled fussion. Plenum Press, 1984.

[3] C. E. Clayton, C. Joshi, and F. F. Chen. Ion-trapping saturation of brillouin instability. Physical Review Letters, 51(18):1656, 1983.

[4] S. C. Wilks, W. L. Kruer, J. Denavit, K. G. Estabrook, D. E. Hinkel, D. Kalantar, A. B. Langdon, B. MacGowan, D. S. Montgomery, and E. A. Williams. Nonlinear theory and simulations of stimulated brillouin backscatter in multispecies plamsas. Physical Review Letters, 74:5048, 1995.

[5] B. Gellert and B. Kronast. Investigation of stimulated brillouin scattering under welldefined interaction conditions. Applied Physics B, 32:175, 1983.

[6] B. Gellert and B. Kronast. Investigation of stimulated brillouin scattering under welldefined interaction conditions; part ii. Applied Physics B, 33:29, 1984.

[7] B. I. Cohen, B. F. Lasinski, A. B. Langdon, and E. A. Williams. Resonantly excited nonlinear ion waves. Physics of Plasmas, 4(4):956, 1997. 
[8] S. H. Glenzer, L. M. Divol, R. L. Berger, C. Geddes, R. K. Kirkwood, J. Moody, E. A. Williams, and P. E. Young. Thomson scattering measurements of saturated ion waves in laser fusion plasmas. Physical Review Letters, 86:2565, 2001.

[9] A. A. Andreev and V. T. Tikhonchuk. JETP, 68:1135, 1989.

[10] W. L. Kruer. The Physics of laser plasma interactions. Addison-Wesley Publishing Company, Inc., 1988.

[11] E. M. Lifshitz and L. P. Pitaevskii. Physical Kinetics, volume 10. Pergamon Press, 1979.

[12] Burton D. Fried and Samuel D. Conte. The plasma dispersion function. Academic Press INC., London, 1961.

[13] L. Landau. On the vibrations of the electronic plasma. Journal of Physics USSR, 10(1):25, 1946.

[14] V. E. Zakharov and V. I. Karpman. Soviet Physics-JETP, 16:351, 1962.

[15] G. J. Morales and T. M. O'Neil. Nonlinear frequency shift of an electron plasma wave. Physical Review Letters, 28:417, 1972.

[16] E. A. Williams and B. I. Cohen. Saturation of laser beam energy transfer by nonlinear ion wave damping and frequecncy shifts. In Conference Proceedings APS-DPP01; Model was introduced and solved analytically by referanced., page QP1.146, Long Beach, 2001. 
[17] J. A. Fejer. Scattering of radio waves by an ionized gas in thermal equilibrium. Canadian Journal of Physics, 38:1114, 1960.

[18] B. La Fontaine, H. Baldis, D. M. Villeneuve, J. Dunn, G. D. Enright, J. C. Kieffer, H. Pepin, M. D. Rosen, D. L. Matthews, and S. Maxon. Characterization of laserproduced plasmas by ultraviolet thomson scattering. Physics of Plasmas, 1(7):2329, 1994.

[19] S. H. Glenzer, C. A. Back, K. G. Estabrook, R. Wallace, K. Baker, B. J. MacGowan, B. A. Hammel, R. E Cid, and J. S. De Groot. Observation of two ion-acoustic wavis in a two-species laser-produced plamsa with thomson scattering. Physical Review Letters, 77(8):1496, 1996.

[20] J. Sheffield. Plasma Scattering of Electromagnetic Radiation. Academic, New York, 1975.

[21] John D. Jackson. Classical Electrodynamics. Hamilton Printing Company, thrid edition, 1998.

[22] J. Myatt. Fluctuations and transport in laser plasmas. PhD thesis, University of Alberta, 1997.

[23] D. E. Evans. The effect of impurities on the spectrum of laser light scattered by a plasma. Plasma Physics, 12:573, 1970.

[24] N. K. Moncur, R. P. Johnson, R. G Watt, and R. B. Gibson. Trident: a versatile high-power nd:glass laser facility for inertial confinement fusion experiments. Applied Optics, 34(21):4274, 1995. 
[25] Kms fusion 1979 annual technical report on inertial fusion research. Technical Report KMSF-U1046, KMS Fusion Inc., 1979.

[26] R. P. Johnson. Results of trident beam characterization 8/14/95 - 8/18/95. Technical Report P-24: 97-U-065, LANL, 1/23/1997 1997.

[27] B. S. Bauer, R. P. Drake, K. G. Estabrook, R. G Watt, M. Wilke, and S. A Baker. Detection of ion plasma waves by collective thomson scattering. Physical Review Letters, 74(18):3604, 1995.

[28] C. E. Thomas and L. D. Siebert. Pulse shape generator for laser fusion. Applied Optics, 15:462-465, 1976.

[29] G. B. Zimmerman and W. L. Kruer. Comments plasma physics controlled fusion. 2:51, 1975.

[30] L. J. Suter, A. A. Hauer, L. V. Powers, D. B. Ress, N. Delameter, W. W. Hsing, O. L. Landen, A. R. Thiessen, and R. E. Turner. Modeling and interpretation of nova's symmetry scaling data base. Physical Review Letters, 73:2328, 1994.

[31] L. J. Suter, R. L. Kauffman, C. B. Darrow, A. A. Hauer, H. N. Kronblum, O. L. Landen, T. J. Orzechowski, D. W. Phillion, J. L. Porter, L. V. Powers, A. Richard, M. D. Rosen, A. R. Theissen, and R. J. Wallace. Radiation drive in laser-heated hohlraums. Physics of Plasmas, 3:2057, 1996.

[32] J. C. Fernandez, J. A. Cobble, D. S. Montgomery, M. D. Wilke, and B. B. Afeyan. Observed insensitivity of stimulated raman scattering on electron density. Physics of Plasmas, 7(9):3743, 2000. 
[33] D. S. Montgomery, B. B. Afeyan, J. A. Cobble, J. C. Fernandez, M. D. Wilke, S. H. Glenzer, R. K. Kirkwood, B. J. MacGowan, J. D. Moody, E. L. Lindman, D. H. Munro, B. H. Wilde, H. A. Rose, D. F. DuBois, B. Bezzerides, and H. X. Vu. Evidence of plasma fluctuations and their effect on the growth of stimulated brillouin and stimulated raman scattering in laser plasmas. Physics of Plasmas, 5(5), 1998.

[34] J. D. Moody, B. J. MacGowan, J. E. Rothenberg, R. L. Berger, L. Divol, S. H. Glenzer, R. K. Kirkwood, E. A. Williams, and P. E. Young. Backscatter reduction using combined spatial, temporal, and polarization beam smoothing in long-scatle-length laser plasmas. Physical Review Letters, 86(13):2810, 2001.

[35] S. H. Glenzer, L. J. Suter, R. L. Berger, K. G. Estabrook, B. A. Hammel, R. L. Kauffman, R. K. Kirkwood, B. J. MacGowan, J. D. Moody, J. E. Rothenberg, and R. E. Turner. Hohlraum energetics with smoothed laser beams. Physics of Plasmas, 7(6), 2000.

[36] R. K. Kirkwood, B. J. MacGowan, D. S. Montgomery, B. B. Afeyan, W. L. Kruer, D. M. Pennington, S. C. Wilks, J. D. Moody, K. Wharton, C. A. Back, K. G. Estabrook, S. H. Glenzer, M. A. Blain, R. L. Berger, D. E. Hinkel, B. F. Lasinski, E. A. Williams, D. H. Munro, B. H. Wilde, and C. Rousseaux. Obervation of multiple mechanisms for stimulating ion waves in ignition scale plasmas. Physics of Plasmas, 4(5):1800, 1997.

[37] C. S. Lui, M. N. Rosenbluth, and R. B White. Parametric saattering instabilities in inhomogeneous plasmas. Physical Review Letters, 31(11), 1973.

[38] D. H. Froula, L. Divol, H. A. Baldis, R. L. Berger, D. G. Braun, B. I. Cohen, R. P. John- 
son, D. S. Montgomery, E. A. Williams, and S. H. Glenzer. Observation of ion heating by stimulated brillouin scattered driven ion-acoustic waves using thomson scattering. Physics of Plasmas, 9(11):4709, 2002.

[39] D. H. Froula, L. M. Divol, and S. H. Glenzer. Measurement of nonlinear growth of ionacoustic waves in two-ion-species plasmas with thomson scattering. Physical Review Letters, 88(10):105003, 2002.

[40] R. L. Berger, C.H. Still, E. A. Williams, and A. B. Langdon. On the dominant and subdominant behavior of stimulated raman and brillouin scattering driven by nonuniform laser beams. Physics of Plasmas, 5(12):4337, 1998.

[41] J. A. Cobble, J. C. Fernandez, N. A. Kurnit, D. S. Montgomery, and R. P. Johnson. The spatial location of laser-driven, foward-propagating waves in a national-ignition-facility plasma. Physics of Plasmas, 7(1):323, 2000.

[42] W. Seka, H. A. Baldis, J. Fuchs, S. P. Regan, D. D. Meyerhofer, C. Stoeckl, B. Yaakobi, R. S. Craxton, and R. W. Short. Multibeam stimulated brillouin scattering from hot, solid-target plasmas. Physical Review Letters, 89(17):175002, 2002.

[43] P. W Rambo, S. C. Wilks, and W. L. Kruer. Hybrid particle-in-cell simulations of stimulated brillouin scattering including ion-ion collisions. Physical Review Letters, $79: 83,1997$.

[44] W. L. Kruer. Nonlinear estimates of brillouin scatter in plasma. Physics of Fluids, 23(6):1273, 1980. 
[45] H. X. Vu, D. F. DuBois, and B. Bezzerides. Transient enhancement and detuning of laser-driven parametric instabilities by particle trapping. Physical Review Letters, 86(19):4306, 2001.

[46] L. Divol, B. I. Cohen, E. A. Williams, A. B. Langdon, and B. F. Lasinski. Nonlinear saturation of stimulated brillouin scattering for long time scales. Physical Review Letters, 2002.

[47] H. A. Rose and D. F. DuBois. Laser hot spots and the breakdown of linear instability theory with application to stimulated brillouin scattering. Physical Review Letters, 72:2883, 1994 .

[48] M.J. Herbst, C. E. Clayton, and F. F. Chen. Saturation of brillouin backscatter. Physical Review Letters, 43:1591, 1979.

[49] J. Handke, S. A. Rizvi, and B. Kronast. Nonlinear wave process revealed in the spectra of stimulated brillouin scattering. Physical Review Letters, 51:1660, 1983.

[50] J. E. Bernard and J. Meyer. Measurement of absolute growth rates and saturation phenomena for stimulated brillouin scattering in a co2-laserirradiated plasma. Physical Review Letters, 55:79, 1985.

[51] W. L. Kruer. From fusion to light surfing. Addison-Wesley, Redwood City, CA, 1991.

[52] C. Riconda. Physica Scripta, 84:217, 2000.

[53] C. J. Pawley, H. E. Huey, and N. C. Jr. Luhmann. Observation of the growth and 
saturation of ion waves generated by optical mixing. Physical Review Letters, 49:877, 1982.

[54] J. A. Heikkinen, S. J. Karttunen, and R. R. E. Salomaa. Ion acoustic nonlinearities in stimulated brillouin scattering. Physics of Fluids, 27:707, 1984.

[55] W. Rozmus. The local-global analysis of the stimulated brillouin scattering in the regime of nonlinear sound waves. Physics of Fluids, page 576, 1992.

[56] R. K. Kirkwood, J. D. Moody, A. B. Langdon, B. I. Cohen, E. A. Williams, M. R. Dorr, J. A. Hittinger, R. L. Berger, P. E. Young, L. J. Suter, L. Divol, S. H. Glenzer, and O. L. Landen. Observation of saturation of energy transfer between copropagating beams in a flowing plasma. Physical Review Letters, 89(21):215003, 2002. 


\section{Part V}

Appendix 
Appendix A

"Measurements of non-linear

growth of ion-acoustic waves in

two-ion-species plasmas with

Thomson scattering", D. H.

Froula, et al., Physical Reveiw

Letters, 88 (2002) 


\title{
Measurements of Nonlinear Growth of Ion-Acoustic Waves in Two-Ion-Species Plasmas with Thomson Scattering
}

\author{
D. H. Froula,* L. Divol, and S. H. Glenzer \\ L-399, Lawrence Livermore National Laboratory, University of California, \\ P.O. Box 808, Livermore, California, 94551 \\ (Received 16 October 2001; revised manuscript received 6 February 2002; published 25 February 2002) \\ We report the first Thomson-scattering measurements of the growth of ion-acoustic waves in well- \\ characterized multi-ion-species plasmas consisting of gold and beryllium. We observe that only the beryl- \\ liumlike mode grows, verifying linear kinetic theory. In addition, a twofold increase in ion temperature \\ is measured when ion-acoustic waves are excited to large amplitudes by stimulated Brillouin scattering \\ (SBS). This increase in ion temperature is a strong indication of hot ions due to trapping. We explain \\ the measured SBS reflectivity by nonlinear detuning of the SBS instability due to these trapping effects.
}

DOI: $10.1103 /$ PhysRevLett.88.105003

Indirect drive inertial confinement fusion depends on efficient propagation of high intensity laser beams through multi-ion-species plasmas in closed-geometry hohlraums. Understanding ion-wave growth and the saturation mechanisms in these plasmas is necessary to improve the energy coupling in the fusion capsule and to develop models that will predict stimulated Brillouin scattering (SBS) laser energy losses at future fusion facilities such as the National Ignition Facility. The SBS instability results from the resonant coupling of an intense laser pulse with an ionacoustic wave. The resulting driven ion-acoustic wave has been shown to saturate [1-4]. A possible mechanism to explain ion-wave saturation is frequency detuning by trapping $[1,5]$.

In this Letter, we present the first observations of the growth of ion-acoustic waves in a well-characterized multi-ion-species plasma consisting of $\mathrm{Au}$ and $\mathrm{Be}$. Using Thomson scattering, we directly measure the growth of the ion-acoustic wave responsible for the SBS light. The temporally resolved Thomson-scattering spectra show simultaneously the scattering from thermal ion-acoustic fluctuations and ion-acoustic waves that have been excited to large amplitude by SBS using a $2 \omega$ interaction beam. For this experiment, the solution to the kinetic dispersion relation gives slow and fast ion-acoustic modes which are evident in our Thomson-scattering spectra as fast Be-like and slow Au-like peaks [6]. We observe that only the Be-like mode grows, verifying predictions made by linear kinetic theory. In addition, we use a new technique that measures the relative damping of the slow and fast modes to determine the ion temperature with high accuracy. We measure up to a factor of 2 increase in the ion temperature when ion-acoustic waves are excited by SBS. This measurement of the ion temperature, and its correlation with SBS, is the first direct quantitative evidence of hot ions created by trapping in laser plasmas. Motivated by the observed increase in ion temperature and SBS reflectivity measurements, we developed a model that includes trapping effects, providing the necessary
PACS numbers: $52.25 . \mathrm{Os}, 52.35 . \mathrm{Fp}, 52.38 .-\mathrm{r}, 52.50 . \mathrm{Jm}$

saturation mechanism to give SBS reflectivity values consistent with the experiment.

The experiments used a three-beam configuration at the Trident Laser Facility [7]. The multi-ion-species targets consisted of a mixture of Be with $1 \%, 5 \%$, or $10 \% \mathrm{Au}$ by atomic number which was fabricated by coating a substrate with alternating Au-Be layers of varying thickness [8]. The plasma was produced by a heater beam with $180 \mathrm{~J}$ of $2 \omega(\lambda=527 \mathrm{~nm})$ laser light in a 1.2-ns-long square pulse. The heater beam was focused normal to the target surface (Fig. 1a) using an $f / 6$ lens and a strip line random phase plate. This produced a line focus $(\sim 1000 \times 100 \mu \mathrm{m})$ with an intensity of $10^{14} \mathrm{~W} \mathrm{~cm}^{-2}$ [9]. The targets were cut such that initially an $800 \times 100 \mu \mathrm{m}$ plasma was formed. A separate high energy $2 \omega$ interaction

(c)
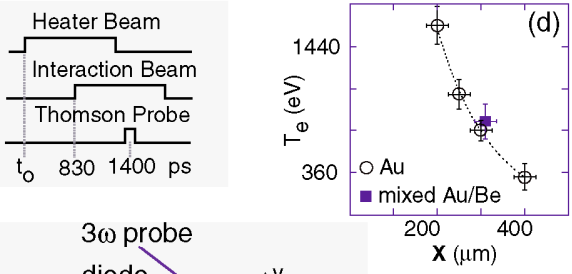

(a)
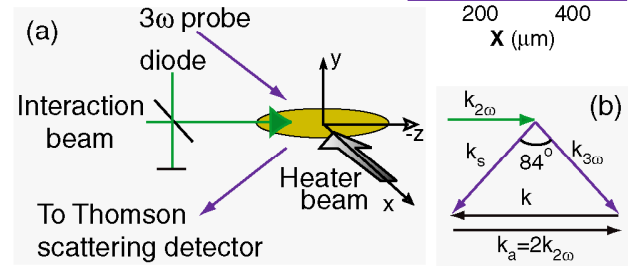

FIG. 1 (color). (a) Schematic of the experimental setup. (b) The angle between the incident $\left(\mathbf{k}_{3 \omega}\right)$ and scattered $\left(\mathbf{k}_{s}\right)$ Thomson beam $\left(\left|k_{3 \omega}\right| \simeq\left|k_{s}\right|\right)$ is determined by matching the driven ion-wave vector $\left(\mathbf{k}_{a} \approx 2 \mathbf{k}_{2 \omega}\right)$ with the Thomsonscattering wave vector (k). (c) Relative beam timings. (d) The electron temperature is plotted as a function of the distance from the target surface at a time of $1.4 \mathrm{~ns}$.

(C) 2002 The American Physical Society

105003-1 
beam, 1.2-ns-long square pulse, was aligned parallel to the target and focused to $\mathrm{a} \sim 60 \mu \mathrm{m}$ diameter spot, resulting in laser intensities up to $4 \times 10^{15} \mathrm{~W} \mathrm{~cm}^{-2}$ [10]. The interaction beam was used to drive SBS which excites ion-acoustic waves in the plasma. The wave number of the excited ion-acoustic wave was matched using a third $3 \omega$ $(\lambda=351 \mathrm{~nm}) 180 \mathrm{ps}$ Thomson-scattering probe beam (Fig. 1b). The beams were timed such that the interaction beam turned on $830 \mathrm{ps}$ after the heater beam. The Thomson-scattering probe began 200 ps after the end of the heater beam (Fig. 1c). A region of plasma overlapping the interaction beam path was imaged onto a half-meter spectrometer with an optical magnification of $5: 1$. The cylindrical Thomson-scattering volume, centered in the $y-z$ plane, was $60 \mu \mathrm{m}$ long and $40 \mu \mathrm{m}$ in diameter. The spectrally resolved Thomson-scattering signal was detected with a streak camera, resulting in a spectral and temporal resolution of $0.05 \mathrm{~nm}$ and $100 \mathrm{ps}$, respectively.

Figure 2 shows the Thomson-scattering data with and without the interaction beam at a distance of $x=300 \mu \mathrm{m}$ from the target surface. The blow-off plasma was well characterized by fitting the standard multi-ion-species form factor $[11,12]$ to the Thomson-scattering spectra taken in the absence of the interaction beam. The electron temperature, $T_{e}(x=300)=600 \mathrm{eV}$, is directly determined by the separation of the fast Be-like mode since the Be ions are fully ionized, $Z_{\mathrm{Be}}=4$. Furthermore, the phase velocity of the slow mode is sensitive to the product of the average Au charge state and electron temperature, $Z_{\mathrm{Au}} T_{e}$, therefore allowing the accurate measure of the charge state, $Z_{\mathrm{Au}}(x=300)=40$. The relative amplitude of the peaks in the Thomson scattering spectra belonging to the slow and fast ion-acoustic waves provides an accurate measure of the ion temperature, $T_{i}(x=300)=$ $250 \mathrm{eV}$. The product of the Au charge state and electron temperature, $Z_{\mathrm{Au}} T_{e}$, is measured along the heater beam

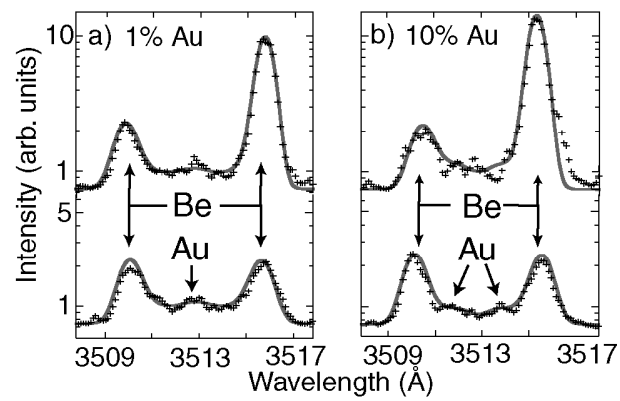

FIG. 2. The measured Thomson-scattering spectra $(+)$ from Be plasmas with (a) $1 \%$ and (b) $10 \% \mathrm{Au}$. The solid line is the best fit theoretical form factor with the addition of a Gaussian line profile in the upper two plots. The bottom data set represents scattering when the interaction beam is turned off, while the upper spectra show data with an interaction beam intensity of $3.5 \times 10^{15} \mathrm{~W} \mathrm{~cm}^{-2}$ axis by varying the distance of the Thomson-scattering volume from the Au disk targets (Fig. 1d). The average Au charge state was assumed to vary slowly from 43 at $x=200 \mu \mathrm{m}$ to 38 at $x=400 \mu \mathrm{m}$ [13].

The wavelength of the probe laser $(\lambda=351 \mathrm{~nm})$, the scattering geometry, and the plasma parameters result in collective Thomson scattering from fluctuations characterized by wave numbers such that the scattering parameter is greater than one, $\alpha=1 / k \lambda_{D} \approx 3$, where $k$ is the thermal Thomson-scattering wave vector and $\lambda_{D}$ is the electron Debye length. An electron density of $n_{e} \simeq 10^{20} \mathrm{~cm}^{-3}$ was calculated by radiation-hydrodynamic modeling and is consistent with other experiments [14]. The collective ion effects are seen in Fig. 2, where representative scattering spectra are shown with two different ion concentrations revealing both the slow and the fast modes.

When the interaction beam is used, ion-acoustic waves are excited by SBS which produce backward scattered light. In order to measure the amplitude of the excited ion waves, the angle $\theta$ between the incident and scattered wave vectors of the Thomson-scattering probe was adjusted to match the Thomson-scattering wave vector, $k \simeq$ $2 k_{3 \omega} \sin \theta / 2$ with the driven ion-wave vector $k_{a} \simeq 2 k_{2 \omega}$ giving $\theta=84^{\circ}$ (Fig. 1b). It is noted that we observe thermal ion-acoustic wave fluctuations in all the Thomson spectra, driven or undriven, as the blueshifted peaks.

The top spectra in Fig. 2 show that, once the interaction beam is turned on, only the redshifted peak belonging to the fast Be-like mode are excited by SBS. No excitation of the slow ion-acoustic wave has been observed. The spectra are fit using the standard theoretical form factor; when SBS is excited, a Gaussian line profile centered on the frequency of the fast Be-like peak is added. The area under the Gaussian line profile is varied to fit the driven peak. In the parameter regime of this experiment, the spatial growth of SBS from either acoustic mode is inversely proportional to its Landau damping. Because of the fast energy equilibration time between the Be and $\mathrm{Au}$, we can assume each species has the same ion temperature. The solution to the multi-ion-species dispersion relation shows that the fast mode is weakly damped (the normalized Landau damping is $\nu_{L} / \omega_{a}=0.05$ for a $10 \%$ Au mixture with $T_{i} / T_{e} \approx 0.3$ ) and the slow mode is heavily damped $\left(\nu_{L} / \omega_{a}=0.3\right)$. Our SBS linear gain calculations, using the codes LIP/PIRANAH [15], reproduce the findings of Fig. 2 with almost no amplification of the slow mode $\left(G_{\mathrm{SBS}} \approx 5\right)$ and a large amplification for the fast mode $\left(G_{\mathrm{SBS}} \approx 200\right)$.

Figure 3 shows the measured SBS reflectivity for various mixtures of $\mathrm{Au}$ and $\mathrm{Be}$. These measurements have been performed by collecting and collimating the SBS light using the $f / 6$ aspheric lens that focuses the interaction beam. The light is then reflected off a beam splitter, passed through a narrow $532 \mathrm{~nm}$ notch filter, and focused onto a diode that measured both the incident and backreflected SBS light (Fig. 1a). We observe the SBS reflectivity to be a few percent, while linear theory (i.e., convective gain) 


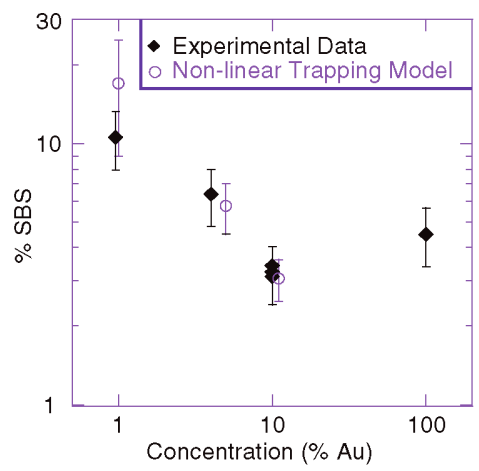

FIG. 3 (color). The SBS reflectivity has been measured to decrease for beryllium plasmas with increasing gold concentration (black diamonds). This trend is reproduced by our nonlinear trapping theory (blue circles).

gives a reflectivity of nearly $100 \%$. It is therefore clear that, while our linear theory predicts the growth of the fast mode and not the slow mode as seen in Fig. 2, the SBS reflectivity data indicate a need for a nonlinear model that includes saturation of ion-acoustic waves.

Figure $4 \mathrm{a}$ shows the ion temperature increase as a function of the SBS excited ion-acoustic wave amplitude. The ion-wave amplitude is defined to be the square root of the ratio of the area under the redshifted spectra, to the area under the blueshifted spectra; the ion-wave amplitude is proportional to $\delta n / n_{e}$. Figure $4 \mathrm{~b}$ indicates the sensitivity of the Thomson spectra to ion temperature. As the ion temperature increases, the damping of the fast mode increases while the damping of the slow mode decreases, resulting in a respective rise and fall in the blueshifted peaks of the thermal undriven Thomson spectra. With the interaction beam employed, the ion-wave amplitude increases and the ion temperature rises (Fig. 4a), while we found no change in the electron temperature. This direct observation of an increase in ion temperature indicates that ion trapping is important and could be the critical saturation mechanism.

In this experiment we excite ion-acoustic waves in the direction of the interaction beam. These driven ion waves trap ions which are accelerated (creating hot ions) in the same direction as the driven acoustic wave. After thermalization this will increase the average ion temperature which we measure from the blueshifted peaks in the Thomson-scattering spectrum; note that the blueshifted peaks result from scattering off ion-acoustic wave fluctuations in the direction counterpropagating to the original hot ions. We have therefore included these trapping effects in our nonlinear theory of SBS.

In our range of experimental parameters, $6<$ $Z_{\mathrm{Be}} T_{e} / T_{i}<14$, the population of $\mathrm{Be}$ ions near the phase velocity of the ion-acoustic wave is large enough so that trapping effects are a plausible saturation mechanism. Trapping reduces the ionic part of the linear Landau
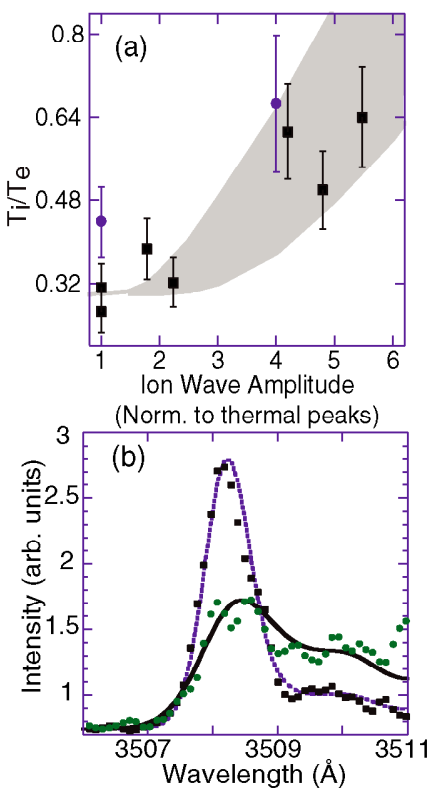

FIG 4 (color), (a) The ratio of $T_{i} / T_{\text {e }}$ as a function of ionwave amplitude is plotted indicating hot ions and ion trapping. Data for Be with $5 \%$ Au (blue circles) and $10 \%$ Au (black squares) are shown. The solid green line is the result of modeling and shows good agreement with the experiment (b) The blueshifted (thermal) Thomson-scattering spectra for a $10 \% \mathrm{Au}$ mixture are shown for a shot without the interaction beam (black squares) and a shot with an interaction beam in tensity of $3.5 \times 10^{15} \mathrm{~W} \mathrm{~cm}^{-2}$ (green circles) resulting in an increase in ion temperature from 250 to $475 \mathrm{eV}$.

damping of the driven wave by flattening the distribution function around the phase velocity of the wave $[5,16]$; therefore, a lower limit for the residual damping, $\nu$, is the electronic Landau damping and a small collisional damping $\left(\nu / \omega_{s} \approx 0.015\right)$.

Another effect of the trapping is a modification of the dispersion relation for the SBS acoustic wave, creating a nonlinear frequency shift, $\delta \Omega[5,17,18]$ :

$$
\begin{aligned}
\frac{\delta \Omega}{\omega} & \approx-\eta\left(\frac{\delta n}{n_{e}}\right)^{1 / 2} \\
& =-\frac{f_{\mathrm{Be}}}{\sqrt{2 \pi}}\left(v^{4}-v^{2}\right) e^{-v^{2} / 2} \sqrt{Z_{\mathrm{Be}} \frac{T_{e}}{T_{i}}}\left(\frac{\delta n}{n_{e}}\right)^{1 / 2},
\end{aligned}
$$

where $\eta$ is the detuning parameter, $\omega_{a}$ is the SBS acoustic wave frequency, $f_{\mathrm{Be}}$ is the fraction of Be ions in the plasma $\left(f_{\mathrm{Be}}=0.9\right)$, and $v=v_{\phi} / v_{\mathrm{Be}}, v_{\mathrm{Be}}=\sqrt{\frac{T_{i}}{M_{B e}}}$ is the thermal velocity of the Be ions. The phase velocity $\left(v_{\phi}\right)$ of the fast mode is given by the multi-ion-species dispersion relation $\left(v^{2}\right.$ is of order of $\left.Z_{\mathrm{Be}} T_{e} / T_{i}\right)$. This nonlinear 
frequency shift detunes the resonant coupling between the interaction beam and the ion-acoustic wave, therefore saturating the SBS instability.

Including these effects in a steady-state coupled-wave model of SBS gives (from Ref. [19]):

$$
\begin{gathered}
c \sqrt{1-\frac{n_{e}}{n_{c}}} \partial_{z} A_{1}(z)=-\frac{i \omega_{0}}{4} \frac{\delta n(z)^{*}}{n_{e}} \frac{n_{e}}{n_{c}} A_{0} \\
\left(i \eta \sqrt{\frac{\delta n}{n_{e}}}+\nu\right) \frac{\delta n(z)}{n_{e}}=\frac{i \omega_{0}^{2}}{\omega_{a}} \frac{Z_{\mathrm{Be}} m_{e}}{m_{i}}\left(\frac{v_{0}}{c}\right)^{2} \frac{A_{1}(z)^{*}}{A_{0}}
\end{gathered}
$$

where $A_{0}\left(A_{1}\right)$ is the incoming (reflected) laser light vector potentials, $\omega_{0}$ is the laser frequency, and $v_{0}$ is the electron quiver velocity. Balancing the two terms on the left-hand side of Eq. (3) shows that detuning becomes important for SBS saturation when $\delta n / n_{e} \approx \nu^{2} / \eta^{2} \simeq$ $0.1 \%$ for our parameters. The SBS reflectivity is calculated by integrating the system of equations [Eqs. (2) and (3)] between $z=0$ and $z=L$ for different fractions of $\Lambda \mathrm{u}$ and is in rough agreement with the experimental values (Fig. 3). Parameters used in the model were selected to match the experiment $\left(\mathrm{L}-800 \mu \mathrm{m}, T_{i} / T_{e} \approx 1 / 2, I_{0}-\right.$ $3 \times 10^{15} \mathrm{~W} \mathrm{~cm}^{-2}, n_{e}=10^{20} \mathrm{~cm}^{-3}$ ). This model is dependent on the electron density and the interaction beam intensity; the error bars in Fig. 3 result from uncertainties in these experimental parameters. The scaling with the fraction of $\mathrm{Au}$ ions is well reproduced, and is mainly from a decreasing phase velocity of the fast mode when the fraction of $\mathrm{Au}$ ions is increased, which increases the number of ions at the phase velocity resulting in a larger detuning parameter $(\eta)$ and therefore more efficient detuning, (i.e., the same acoustic wave amplitude creates a larger frequency shift).

We further test this model by estimating the ion temperature in the Thomson-scattering volume $(z=L / 2)$. We balance the energy flux deposited into the acoustic waves (from the Manley-Rowe relations) with a free streaming heat flux [20]:

$$
\frac{\omega_{s}}{\omega_{0}}\left(R_{\mathrm{SBS}} I_{0}\right)=n_{\mathrm{Be}} T_{i} v_{\mathrm{Be}} .
$$

This model assumes that the energy deposition is local, i.e., the mean-free path of the hot ions accelerated by trapping (a few tens of $\mu \mathrm{m}$ ) is much smaller than the length of the plasma ( $L \approx 1 \mathrm{~mm}$ ), and thermalization is fast enough so that heat is carried away by Maxwellian ions when a steady state is reached. Theoretical and experimental ion-wave amplitudes were related by matching a high reflectivity point; the model then reproduces the experimental scaling for $T_{i}$. The gray area in Fig. $4 \mathrm{a}$ is due to the error in the modeled SBS reflectivity and the uncertainties in the energy loss to the electrons and $\mathrm{Au}$ ions.

Validation of the main assumptions of this modeling (a steady state is reached and the nonlinear frequency shift is the main effect that detunes and saturates the instability) through comparisons of particle-in-cell and fluid simulations is beyond the scope of this paper, but such a detuning effect has been emphasized in various publications $[1,3,21]$. The saturation mechanism governed by detuning will be much weaker in high- $Z$ plasmas $(\mathrm{CO} 2$, pure $\mathrm{Au}$ ) because the detuning parameter is a strong function of charge state.

In summary, we have presented the first measurements of the growth of ion-acoustic waves in well-characterized multi-ion-species plasmas. Our measurements of the SBS reflectivity indicate the need for a saturation mechanism. Using Thomson scattering and by adding a small fraction of $\mathrm{Au}$ ions to a Be plasma enabled the first accurate measurement of $T_{i}$ in laser-plasma interaction studies; while $T_{i}$ was found to increase with SBS reflectivity and with the amplitude of the (local) acoustic waves, $T_{e}$ was found to be constant. This is consistent with our model, where the increase in $T_{i}$ is due to trapping of ions by the SBS ion-acoustic waves

We would like to acknowledge the efforts of the Trident laser crew: R. Johnson, T. Hurry, R. Gonzales, N. Okamoto, F. Archulata, S. Letzring, and R. Perea We thank D. Montgomery, C. Labaune, E. Williams, B Cohen, R. Berger, and $\mathrm{H}$. Baldis for valuable discussions. We further thank R. Scalettar and the UC Davis Physics Department for their support. This work was performed under the auspices of the U.S. Department of Energy by the Lawrence Livermore National Laboratory under Contract No. W-7405-ENG-48.

*Also at Physics Department, University of California at Davis, California 95616

[1] C. E. Clayton et al., Phys. Rev. Lett. 51, 1656 (1983).

[2] B. Gellert and B. Kronast, Appl. Phys. B 32, 175 (1983)

[3] S. C. Wilks et al., Phys. Rev. Lett. 74, 5048 (1995).

[4] S. H. Glenzer et al., Phys. Rev. Lett. 86, 2565 (2000).

[5] B. I. Cohen et al., Phys. Plasmas 4, 956 (1997).

[6] E. A. Williams et al., Phys. Plasmas 2, 129 (1995).

[7] N. K. Moncur et al., Appl. Opt. 34, 4274 (1995).

[8] S. H. Glenzer et al., Phys. Rev. Lett. 77, 1496 (1996).

[9] B. S. Bauer et al., Phys. Rev. Lett. 74, 3604 (1995).

[10] R. P. Johnson (private communication).

[11] J. A. Fejer, Can. J. Phys. 38, 1114 (1960).

[12] D. E. Evans, Plasma Phys. 12, 573 (1970)

[13] M. E. Foord et al. Phys. Rev. Lett. 85, 992 (2000).

[14] D. S. Montgomery et al., Phys. Rev. Lett. 84, 678 (2000).

[15] L. V. Powers et al., Phys. Plasmas 2, 2473 (1995).

[16] V.E. Zakharov and V. I. Karpman, Sov. Phys. JETP 16, 351 (1962).

[17] G. J. Morales and T. M. O'Neil, Phys. Rev. Lell. 28, 417 (1972).

[18] A. A. Andreev and V. T. Tikhonchuk, JETP 68, 1135 (1989).

[19] E. A. Williams and B. I. Cohen, in Proceedings of the APSDPP01, Long Beach, CA, 2001, p. QP1.146.

[20] W. L. Kruer, Phys. Fluids 23, 1273 (1980)

[21] H. X. Vu et al., Phys. Rev. Lett. 86, 4306 (2001).

$105003-4$ 
Appendix B

"Obervation of ion heating by stimulated-Brillouin-scattereddriven ion-acoustic waves using Thomson scattering", D. H. Froula, et al., Physics of Plasmas, 9(10) (2002) 


\section{Observation of ion heating by stimulated-Brillouin-scattering-driven ion-acoustic waves using Thomson scattering}

D. H. Froula and L. Divol

Lawrence Livermore National Laboratory, University of California, P.O. Box 808,

livermore, California 94551

and University of California at Davis, California 95616

H. A. Baldis

Institute for Lasers Science Applications, University of California, California 94551

R. L. Berger, D. G. Braun, and B. I. Cohen

Lawrence Livermore National Laboratory, University of California, P.O. Box 808,

Livermore, California 94551

R. P. Johnson and D. S. Montgomery

Los Alamos National Laboratory, Los Alamos, New Mexico 87545

E. A. Williams and S. H. Glenzer

Lawrence Livermore National Laboratory, University of California, P.O. Box 808 Livermore, California 94551

(Received 3 April 2002; accepted 26 July 2002)

Thomson-scattering measurements of the growth of ion-acoustic waves are obtained from well-characterized multi-ion-species plasmas consisting of gold and beryllium. Simultaneously, the ion temperature is accurately measured by comparing the relative amplitude of thermal ion-acoustic modes. A twofold increase in ion temperature was measured when ion-acoustic waves are excited to large amplitudes by stimulated Brillouin scattering (SBS). This increase in ion temperature is a strong indication of hot ions due to trapping. The measured SBS reflectivity is explained by nonlinear detuning of the SBS instability due to these trapping effects. (C) 2002 American Institute of Physics. [DOI: 10.1063/1.1508776]

\section{INTRODUCTION}

Ion-acoustic wave saturation mechanisms in large scale multi-ion-species plasmas play an important role in the energy coupling into the fusion capsule. It is therefore imperative to the success of inertial confinement fusion (ICF) to understand the physical processes and to develop models ${ }^{1,2}$ that will predict ion-acoustic wave growth and stimulated Brillouin scattering (SBS) laser energy losses at future fusion facilities such as the National Ignition Facility (NIF). The SBS instability results from the resonant coupling of an intense laser pulse with an ion-acoustic wave. The resulting reflected SBS light is dependent on the ion-acoustic waves excited by the SBS instability. The amplitude of the SBS driven ion-acoustic waves have been shown to saturate. ${ }^{3-6} \mathrm{~A}$ possible mechanism to explain the saturation of the SBS instability is frequency detuning by trapping. ${ }^{3,7}$

We present observations of the growth of ion-acoustic waves in a well-characterized multi-ion-species plasma consisting of Au and Be. Using Thomson scattering, we directly measured the growth of the ion-acoustic waves responsible for the SBS light; concurrently we measure the SBS reflectivity. The Thomson-scattering spectra show simultaneously the scattering from thermal ion-acoustic fluctuations and ionacoustic waves that have been excited to large amplitude by SBS using a $2 \omega(\lambda=527 \mathrm{~nm}$ frequency doubled from $\lambda$ $=1054 \mathrm{~nm}$ ) interaction beam. For this experiment, the addi- tion of small amounts of $\mathrm{Au}$ to Be plasmas results in a solution to the kinetic dispersion relation with two modes. The mode with the larger phase velocity is weakly damped while the other mode is heavily damped. In this manuscript we call "fast mode" the solution to the kinetic dispersion relation with the larger phase velocity while the "slow mode" is the solution with the smaller phase velocity.

We observe that when exciting SBS, only the fast mode grows to large amplitude verifying predictions made by linear kinetic theory. In addition, we use a new technique that measures the relative damping of the two modes to determine the ion temperature with high accuracy. We measure up to a factor of two increase in the ion temperature when ionacoustic waves are excited by SBS. This measurement of an increase in ion temperature, and its correlation with SBS, is direct quantitative evidence of hot ions created by trapping in laser plasmas. Motivated by this observed increase in ion temperature and our SBS reflectivity measurements, we developed a model that includes trapping effects, providing a saturation mechanism that gives SBS reflectivity values consistent with the experiment.

In Sec. II of this paper we present the experimental setup. Section III describes the plasma characteristics for both pure $\mathrm{Au}$ and $\mathrm{Be}-\mathrm{Au}$ plasmas. Section IV discusses our experimental Thomson scattering and SBS reflectivity results. A theoretical modeling that includes nonlinear detuning due to trapping effects is developed and compared to our 


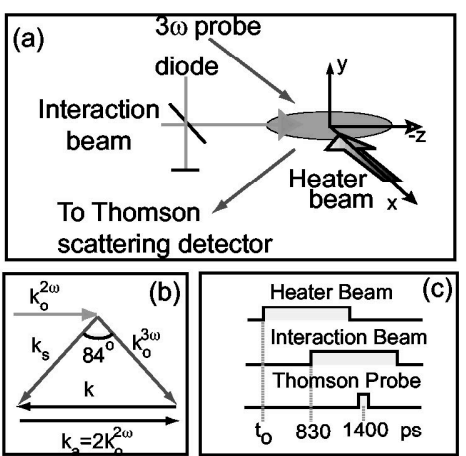

FIG. 1. (a) Schematic of the experimental setup. (b) The angle between the incident $\left(\mathbf{k}_{o}^{3 \omega}\right)$ and scattered $\left(\mathbf{k}_{s}^{3 \omega}\right)$ Thomson beam $\left(\left|k_{o}^{3 \omega}\right| \simeq\left|k_{s}^{3 \omega}\right|\right)$ is determined by matching the driven ion-wave vector $\left(\mathbf{k}_{\alpha} \simeq 2 \mathbf{k}_{o}^{2 \omega}\right)$ with the Thomson-scattering wave vector (k). (c) Relative beam timings.

cxpcrimental results in Scc. V. Scction VI summarizes our findings.

\section{EXPERIMENTAL SETUP}

\section{A. Target and beam configuration}

The experiments used a three-beam configuration at the Trident Laser Facility. ${ }^{8}$ The targets consisted of pure $\Lambda \mathbf{u}$ or a mixture of $\mathrm{Be}$ with $1 \%, 5 \%$, or $10 \% \mathrm{Au}$ by atomic number. These multi-ion-species targets were fabricated by coating a substrate with alternating $\mathrm{Au}-\mathrm{Be}$ layers of varying thickness. $\mathrm{L} A \mathrm{SNEX},{ }^{9-11}$ a hydrodynamic code using an atomic physics model that has been tested in Ref. 12 , calculates that plasmas created with this target configuration homogeneously mix within the first nanosecond of the experiment.

The plasma was produced by a heater beam with $180 \mathrm{~J}$ of $2 \omega(\lambda=527 \mathrm{~nm})$ laser light in a $1.2 \mathrm{~ns}$-long square pulse. The heater beam was focused normal to the target surface [Fig. 1(a)] using an $f / 6$ lens and a strip line random phase plate (RPP). This produced a line focus $(\sim 1000 \times 100 \mu \mathrm{m})$ with an intensity of $10^{14} \mathrm{~W} \mathrm{~cm}^{-2} .13$ The targets were cut such that initially an $800 \times 100 \mu \mathrm{m}$ plasma was formed.

A separate high energy ( $200 \mathrm{~J}) 2 \omega$ interaction beam, with a $1.2 \mathrm{~ns}-$ long square pulse, was aligned parallel to the target and focused with an $f / 6$ lens to a $\sim 60 \mu \mathrm{m}$ diameter spot, resulting in laser intensities up to $7 \times 10^{15} \mathrm{~W} \mathrm{~cm}^{-2}$. The interaction beam was used to drive SBS which excites ionacoustic waves in the plasma. A third $3 \omega(\lambda=351 \mathrm{~nm}) 180$ ps long beam was used as a Thomson-scattering probe. The geometry was selected so that the light from the Thomson probe would scatter from ion-acoustic waves driven by the interaction beam [Fig. 1(b)]. The beams were timed such that the interaction beam turned on $830 \mathrm{ps}$ after the heater beam. The Thomson-scattering probe began $200 \mathrm{ps}$ after the end of the heater beam [Fig. 1(c)]; there is an uncertainty in the absolute timing of $150 \mathrm{ps}$ between the end of the heater beam and the peak of the Thomson probe.

\section{B. Stimulated Brillouin scattering}

The SBS instability results from the resonant coupling of the incident electromagnetic wave $\left(\omega_{o}^{2 \omega}, \mathbf{k}_{o}^{2 \omega}\right)$ with an ionacoustic wave $\left(\omega_{a}, \mathbf{k}_{a}\right)$ and a back-scattered electromagnetic wave $\left(\omega_{o}^{2 \omega}-\omega_{a},-\mathbf{k}_{o}^{2 \omega}\right)$. The beating of the incident and scattered wave creates a ponderomotive force which resonantly drives the acoustic wave $\left(\mathbf{k}_{a} \simeq 2 \mathbf{k}_{o}^{2 \omega}\right)$. In turn, the growing ion-acoustic wave Bragg scatters the incident wave, causing the scattered wave to grow. The resonanting instability causes the ion waves to grow from thermal noise levels to large amplitude.

In this experiment, the backward SBS light has been collected by the lens used to focus the interaction beam. A $4 \%$ beam splitter separated the back-scattered light which was then filtered with a narrow $527 \mathrm{~nm}$ notch filter used to eliminate stray light. The SBS reflected light and the interaction beam power were monitored by a diode which enables an absolute measurement of the SBS reflectivity [Fig. 1(a)].

\section{Thomson scattering}

The wavelength of the probe laser $(\lambda=351 \mathrm{~nm})$, the scattering geometry, and the plasma parameters result in collective Thomson scattering from fluctuations characterized by wave numbers such that the scattering parameter is greater than one, $\alpha=1 / k_{a} \lambda_{D} \approx 3$, where $k_{a}$ is the acoustic scattering wave vector and $\lambda_{D}$ is the electron Debye length. The electron density was calculated using LASNLX to be $n_{e}(x=300 \mu \mathrm{m}) \simeq 10^{20} \mathrm{~cm}^{-3}$ which is consistent with Refs. 14 and $15 ; x$ is the dircetion of the target normal [sec Fig. 1(a)]. In these calculations, nonlocal thermodynamic equilibrium atomic physics was uscd with a flux limitcr of $f$ $=0.03$. Thomson scattering was used as a diagnostic to measurc the relative amplitude of the SBS driven ion-acoustic waves by directly scattering from ion-acoustic fluctuations with a wave vector $\mathbf{k}_{a} \simeq 2 \mathbf{k}_{o}^{2 \omega}$. Figurc 1 (b) shows the wave number matching condition for Thomson scattering from the SBS driven acoustic waves. This relation is used to detcrmine the angle $\theta$ between the incident light and the Thomson-scattering collection optics

$$
k_{a}=2 k_{3 \omega} \sin \theta / 2 \Rightarrow \theta=84^{\circ} .
$$

The Thomson-scattering spectra show peaks corresponding to the modes of the plasma which are solutions to the plasma dispersion relation. For a single-ion-species plasma and $\alpha>1$ the Thomson-scattering spectrum shows two peaks near the Thomson probe laser frequency corresponding to the co- and counter-propagating ion-acoustic waves with a wave number equal to $\mathbf{k}_{a}$ [Fig. 1(b)]. In the presence of the interaction beam only the ion-acoustic wave co-propagating in the direction of the interaction beam will be excited; therefore, only the red-shifted peak will be amplified.

A region of plasma overlapping the interaction beam path was imaged onto a half-meter spectrometer with an optical magnification of 5:1. The cylindrical Thomsonscattering volume was defined by the $f / 10$ focusing optics and the projection of a $200 \mu \mathrm{m} \times 200 \mu \mathrm{m}$ aperture defined by the spectrometer and streak camera slits. The Thomsonscattering volume is centered with respect to the target sur- 

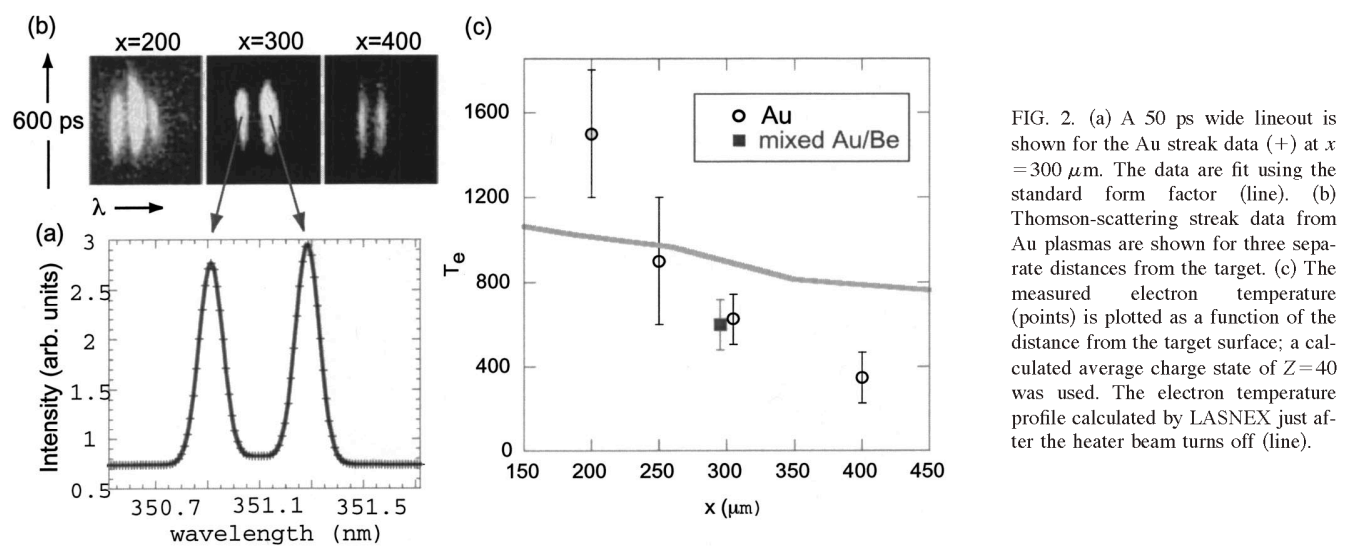

face $(y-z$ plane) and is approximately $40 \mu \mathrm{m} \times 40 \mu \mathrm{m}$ $\times 60 \mu \mathrm{m}$. Its distance from the target surface was varied for the gold plasmas between $x=200 \mu \mathrm{m}$ and $x=400 \mu \mathrm{m}$; for the gold-beryllium targets the volume probed was at a distance of $x=300 \mu \mathrm{m}$. The spectrally resolved Thomsonscattering signal was detected with a Hamamatsu S-20 streak camera resulting in a spectral and temporal resolution of 0.04 $\mathrm{nm}$ and $100 \mathrm{ps}$, respectively.

\section{PLASMA CHARACTERIZATION}

\section{A. Gold plasmas}

The linear dispersion relation for ion-acoustic waves in a single-ion-species plasma is given by

$$
\left(\frac{\omega_{a}}{k_{a}}\right)^{2}=\frac{k_{b} T_{e}}{M}\left(\frac{Z}{1+k^{2} \lambda_{D}^{2}}+\frac{3 T_{i}}{T_{e}}\right),
$$

where $M$ is the ion mass, $Z$ is the average charge state, while $T_{e}$ and $T_{i}$ are the electron and ion temperatures, respectively. For a high- $Z$ plasma or for $T_{i} / T_{e} \ll 1$, a condition that is met in our Au plasmas, the second term in Eq. (2) can be neglected. In this case the frequency separation of the ionacoustic peaks is proportional to the electron temperature. The wavelength separation between the two peaks is $\Delta \lambda$ $=2 \omega_{a} \lambda_{3 \omega}^{2} / 2 \pi c$. Therefore, we find from Eqs. (1) and (2)

$$
\Delta \lambda=4 \lambda_{3 \omega} \sin \left(\frac{\theta}{2}\right) \sqrt{\frac{Z k_{b} T_{e}}{M\left(1+k^{2} \lambda_{D}^{2}\right)}},
$$

which gives, $\Delta \lambda[n m] \simeq 3 \times 10^{-3} \sqrt{Z T_{e}[\mathrm{eV}]}$. This formula can be used to estimate the electron temperature but for better accuracy, our data were fit using the standard theoretical form factor [Fig. 2(a)].

Figure 2(b) shows Thomson-scattering data for Au plasmas at various distances from the target. The product of the $\mathrm{Au}$ charge state and electron temperature, $Z_{\mathrm{Au}} T_{e}$, is measured along the heater beam axis by varying the distance of the Thomson-scattering volume from the Au disk targets. Figure 2(c) is a plot of the electron temperature gradient measured along the $x$ axis using the average Au charge state

$\left(Z_{\mathrm{Au}}=43\right)$ calculated by LASNEX. The calculated average charge state was flat in the measured region. In this study, these calculations were experimentally verified by measuring the average Au charge state using multi-ion-species plasmas at $x=300 \mu \mathrm{m}$ (Sec. III B). The scattering spectra for the closest measurement to the target $(x=200 \mu \mathrm{m})$ show three distinct peaks; we have assumed that the middle peak is stray light most likely scattered directly from the target surface, and found $T_{e}=1500 \mathrm{eV}$. If on the contrary we interpret one of the outer peaks as spurious, the inferred electron temperature would be $400 \mathrm{eV}$. Figure 2(c) shows the electron temperature profile just after the heater beams are turned off (1.2 ns) as calculated by LASNEX for our experimental conditions. These calculations show strong conduction into the solid target after the heater beam is turned off at $1.2 \mathrm{~ns}$. The discrepancy in the calculated electron temperature far from the target surface could be due to the flux limiter used in the calculation; there are also geometric effects due to the twodimensional (2D) axisymetric hydrodynamic code.

\section{B. Multi-ion-species plasmas}

Figure 3 shows the thermal Thomson-scattering spectra, measured without the interaction beam, for Be plasmas with $1 \%, 5 \%$, and $10 \% \mathrm{Au}$ at a distance of $300 \mu \mathrm{m}$ from the target. The two ion-acoustic modes result in four Thomsonscattering peaks ( 2 modes, counter and co-propagating to the interaction beam axis). The peaks with the greatest separation correspond to a fast ion-acoustic mode $\left(v_{\phi} / c=4.2\right.$ $\times 10^{-4}$ ). The smaller, less shifted peaks correspond to a slow ion-acoustic mode $\left(v_{\phi} / c=9 \times 10^{-5}\right)$. In Fig. 3 four peaks can be seen, except for the $1 \% \mathrm{Au}$ case where the two inner peaks are not resolved by the spectrometer. Indeed the solution to the multi-ion-species dispersion relation shows, for our conditions, the presence of a weakly damped fast mode (the normalized Landau damping is $\nu_{L} / \omega_{a}=0.05$ ) and a slow mode with a larger damping $\left(\nu_{L} / \omega_{a}=0.3\right)$ [Fig. 6(b)].

The contribution of the different particles to each mode 


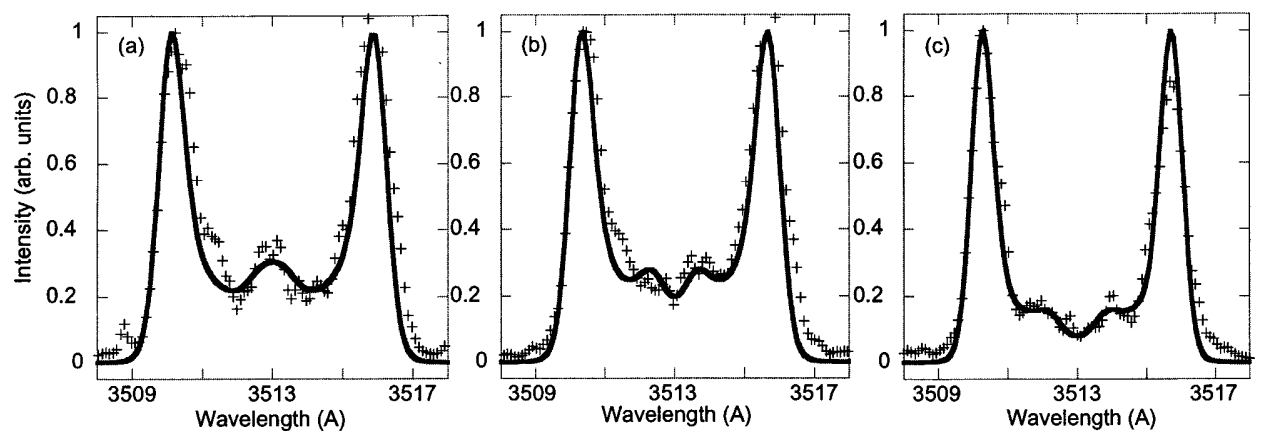

FIG. 3. Thomson-scattering spectra for three different Au concentrations averaged over $\sim 25$ ps are shown: (a) $1 \% \mathrm{Au}$, (b) $5 \% \mathrm{Au}$, (c) $10 \% \mathrm{Au}$. The solid lines represent the best fit theorctical multi-ion-species form factor.

is apparent through the calculation of the respective species susceptibilities:

$$
\chi_{\alpha}=\frac{\omega_{p_{\alpha}}^{2}}{k^{2}}\left[P \int_{-\infty}^{+\infty} \frac{\frac{\partial f_{o}^{\alpha}}{\partial v}}{v-\frac{\omega}{k}} d v+i \pi \frac{\partial f_{o}^{\alpha}\left(\left(\frac{\omega}{k}\right)^{\alpha}\right)}{\partial v}\right],
$$

where $\omega_{p_{\alpha}}=e^{2} Z_{\alpha}^{2} n_{\alpha} / \varepsilon_{o} m_{\alpha}$ is the plasma frequency of the $\alpha$-specie; $P$ denotes the Cauchy principal value. Figure 4 compares the contribution of the individual species through a plot of the respective susceptibilities. For the slow mode (left column), both the Be and electron species have susceptibilities with a real part $\left(\operatorname{Re}\left(\chi_{\rho, \mathrm{Be}}\right) \simeq\left(k_{a} \lambda_{n e, D \mathrm{Be}}\right)^{-2}\right)$ which

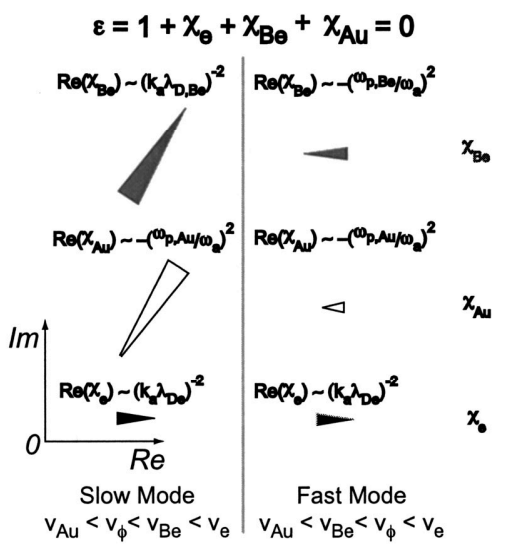

FIG. 4. The magnitude and phase of the susceptibility for each species is shown for both the slow and fast modes by the amplitude and angle of the corresponding arrow. In the slow mode, the Be ions screen (top left $\operatorname{Re}\left(\chi_{\mathrm{Be}}\right)>0$ ) the motion of the $\mathrm{Au}$ ions (middle left $\operatorname{Re}\left(\chi_{\mathrm{Au}}\right)<0$ ). The $\mathrm{Au}$ ions participate very little in the fast mode (middle right $\left|\chi_{\mathrm{Be}}\right| \leqslant 1$ ). In both modes the electrons have a screening effect (bottom row). There is a small imaoinary component in the susceptibilities of the fast mode, therefore, the mode is weakly damped (right side). The slow mode has significan comributions along the imaginary axis resulting in large damping. gives a real vector component opposing that of the $\mathrm{Au}$ species; therefore, both the electron and Be ion motion screen the Au ion fluctuations. The right column of Fig. 4 shows the respective contribution of the species to the fast mode. Here both the $\mathrm{Be}$ and $\mathrm{Au}$ ions participate in the wave (i.e., both have real vector components in the same direction and $\left.\operatorname{Re}\left(\chi_{\mathrm{Be}, \mathrm{Au}}\right) \simeq-\omega_{p, \mathrm{Au}, \mathrm{Be}}^{2} / w_{a}^{2}\right)$, while the electron motion screens the fields setup by the ions. For the fast mode, using the conditions similar to the experiment $\left(T_{e}=430 \mathrm{eV}, T_{i}\right.$ $=140 \mathrm{eV}, n_{e}=10^{20} \mathrm{~cm}^{-3}$, and $\left.Z_{\mathrm{Au}}=40\right)$ we find: $\chi_{\mathrm{Be}}$ $=-6.4+0.06 i ; \chi_{\mathrm{Au}}=-2.06-0.17 i ; \chi_{e}=7.43+0.136 i$ (left column of Fig. 4). Noting that the phase velocity of the fast mode is much larger than the thermal velocities of both types of ions $\left(v_{\mathrm{Be}} / c=1.3 \times 10^{-4}, v_{\mathrm{Au}} / c=2.8 \times 10^{-5}\right)$, the fast mode consists of $\mathrm{Be}$ and $\mathrm{Au}$ ions oscillating in phase $\left(\operatorname{Re}\left(\chi_{\mathrm{Be}, \mathrm{Au}}\right) \sim-\omega_{p, \mathrm{Au}, \mathrm{Be}}^{2} / \omega_{a}^{2}\right)$ while being shielded by the electrons $\left(\operatorname{Re}\left(\chi_{e}\right) \simeq\left(k_{a} \lambda_{D e}\right)^{-2}\right)$. This is in contrast to the slow mode (right column of Fig. 4) where the phase velocity is between the thermal velocities of the individual ions resulting in susceptibilities $\left(\chi_{\mathrm{Be}}-26.7+41 i ; \chi_{\mathrm{Au}}--35.2\right.$ $-42.9 i ; \chi_{e}=7.43+0.03 i$ ) that show the ions oscillating out of phase. ${ }^{16,17}$ Here the Be ions play a role similar to the electrons in shielding the $\mathrm{Au}$ ions $\left(\operatorname{Re}\left(\chi_{\mathrm{Be}}\right) \simeq\left(k_{a} \lambda_{D, \mathrm{Be}}\right)^{-2}\right.$, where $\left.\lambda_{D, \mathrm{Be}}=v_{\mathrm{Be}} / \omega_{p, \mathrm{Be}}\right)$. The individual particle contributions to the susceptibilities for each mode are shown in Fig. 4.

As in the single species case, the separation between the peaks corresponding to a particular mode is roughly proportional to the phase velocity of the mode, and therefore, if the charge state of one of the species is known then the electron temperature and charge state of the second species can be measured.

The Thomson-scattering spectra were fit using the standard multi-ion-species form factor first developed by Fejer ${ }^{18}$ and later applied by Evans. ${ }^{19}$ The electron temperature, $T_{e}(x=300 \mu \mathrm{m})=600 \mathrm{eV}$, is directly determined from this fit since at this temperature, $\mathrm{Be}$ is fully ionized, $Z_{\mathrm{Be}}=4$. Therefore, the use of two-ion-species plasmas allows the direct measure of the average gold charge state, $Z_{\mathrm{Au}}(x$ $=300 \mu \mathrm{m})=40$, verifying our LASNEX calculations. Figure 5 (a) shows the sensitivity of the form factor to electron tem- 

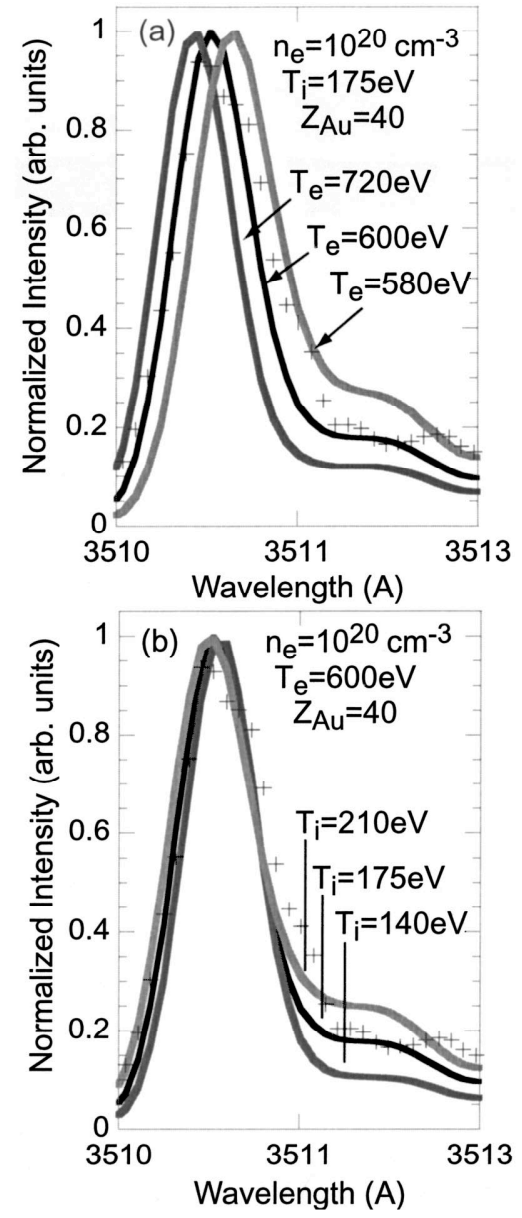

FIG. 5. (a) The theoretical form factor is plotted for $20 \%$ variations in electron temperature (a), and ion temperature (b) while all other parameters are held fixed. The spectra are normalized such that their maximum amplitude is one.

perature. It is clear from these plots that the electron temperature can be determined to better than $15 \%$.

Furthermore, the two-ion-species plasmas have allowed us to measure the ion temperature in a unique way; the relative amplitude of the peaks in the Thomson-scattering spectra belonging to the slow and fast ion-acoustic waves provide an accurate measure of the ion temperature, $T_{i}(x$ $=300 \mu \mathrm{m})=250 \mathrm{eV}$. As a result of the sensitivity of Landau damping to ion temperature (Fig. 6), the damping of the fast mode increases while the damping of the slow mode decreases when the ion temperature is increased. This is illustrated by a respective increase or decrease in the amplitude of the peaks in the Thomson-scattering spectra [Fig. 5(b)].
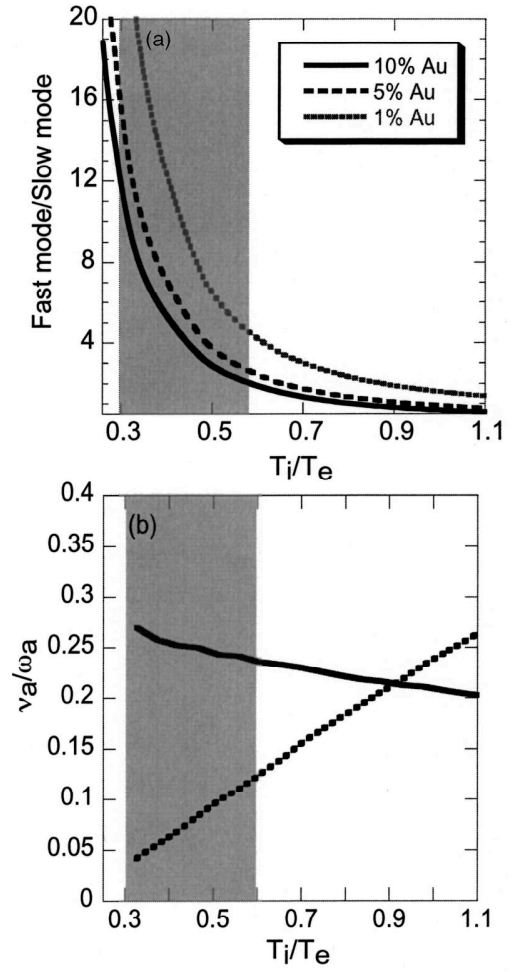

FIG. 6. (a) The ratio between the amplitude of the light scattered from the fast mode to the amplitude of the light scattered from the slow mode is plotted as a function of ion temperature for a fixed electron temperature, $T_{e}=600 \mathrm{eV}$. (b) Normalized Landau damping. $v_{a} / \omega_{a}$. for the fast mode (dashed line) and the slow mode (solid line), as a function of the ion temperature for a fixed electron temperature, $T_{e}=600 \mathrm{eV}$. The concentration of Au was $10 \%$. The shaded regions correspond to the range of ion temperatures in the present experiment where the slow mode is more heavily damped than the fast mode.

Figure 6(a) indicates the sensitivity of the Thomsonscattering spectra to the ion temperature; the ratio between the amplitudes of the slow and fast mode is plotted as a function of ion temperature while all other parameters are held constant. It is possible to measure the ion temperature to better than $15 \%$ using these multi-ion-species plasmas [Fig. $5(\mathrm{~b})]$.

\section{EXPERIMENTAL RESULTS}

\section{A. Thomson scattering on SBS driven ion-acoustic} waves

Figure 7(a) shows the Thomson-scattering data in which ion-acoustic waves are being driven by the ponderomotive force due to the interaction beam. Figure 7 (b) shows lineouts of these data revealing that once the interaction beam is turned on, only the ion-acoustic waves excited by SBS are 

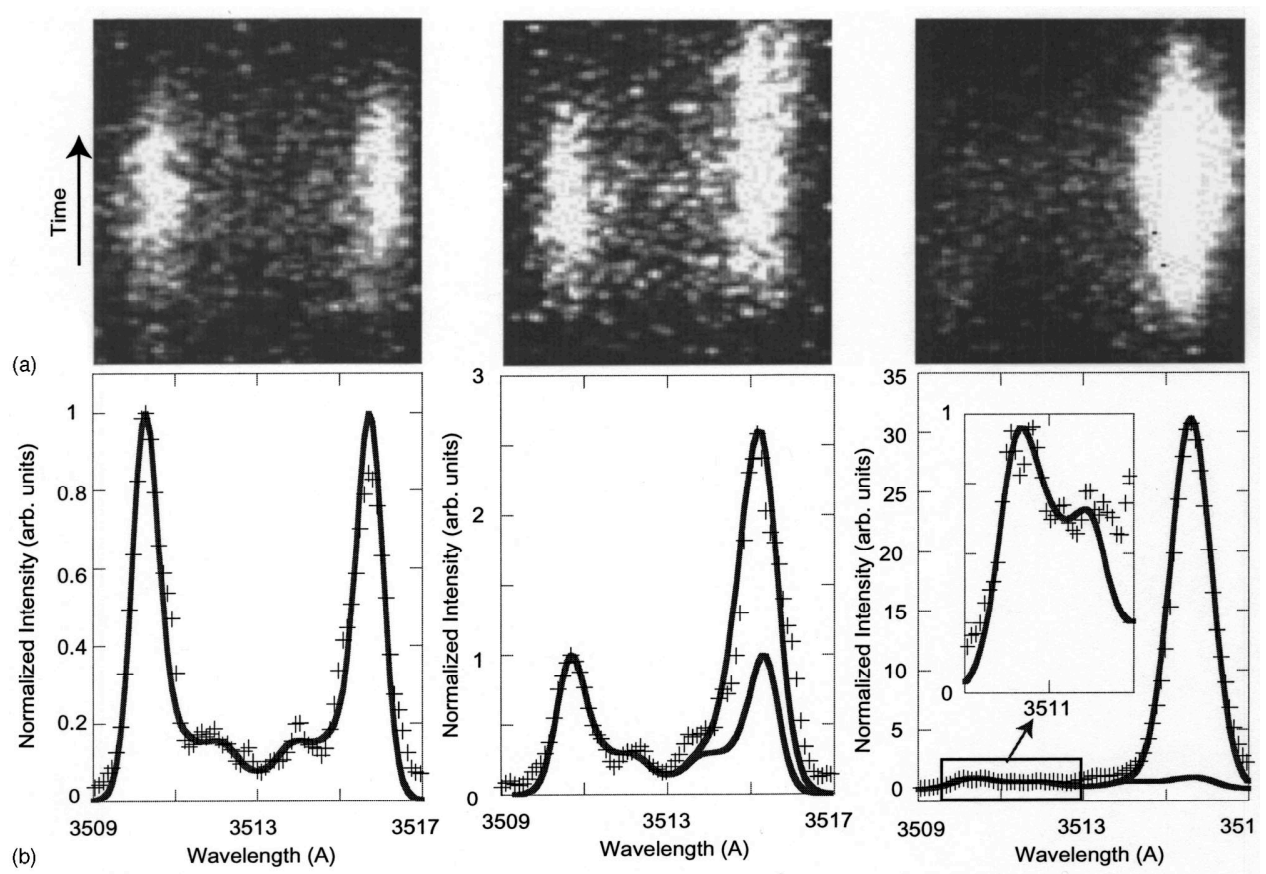

FIG. 7. (a) Streaked Thomson spectra for a Be plasma with a $10 \%$ Au mixture are shown for a shot without the interaction beam (undriven) and shots with interaction beam intensities of $5 \times 10^{15} \mathrm{~W} \mathrm{~cm}^{-2}$ and $7 \times 10^{15} \mathrm{~W} \mathrm{~cm}^{-2}$ (driven). (b) Line outs show the increasing amplitude of the red-shifted light; the square root of the intensity is proportional to the amplitude of the driven ion-acoustic wave. The blue-shifted peaks are fit with the standard multi-ion-species form factor showing an increase in ion temperature from 250 to $475 \mathrm{eV}$.

driven to large amplitudes. The undriven part of the spectra are fit using the standard theoretical form factor; when ionacoustic waves are excited, the driven part of the spectra is fit with a Gaussian. This fit is used to calculate the relative amplitude of the driven acoustic wave. The ion-acoustic wave amplitude is defined to be the square root of the ratio between the area under the red-shifted Thomson-scattering spectra, and the area under the blue-shifted spectra; the ionacoustic wave amplitude is proportional to $\delta n / n_{e}$

Our SBS linear gain calculations, using the codes I.IP/PIRANAH ${ }^{20}$ reproduce the findings of Fig. 7 with almost no amplification of the slow mode $\left(G_{\mathrm{SBS}}^{\text {slow }} \approx 5\right)$ and a large amplification for the fast mode $\left(G_{\mathrm{SBS}}^{\mathrm{fast}} \approx 200\right)$. This difference in the amplification can be explained; when these modes are being driven in steady state, the rate of energy lost through Landau damping $\left(\nu_{L} W_{a}\right)$ must be balanced with the energy transferred to the electrons by the ponderomotive coupling. The energy of the SBS driven acoustic wave is given by

$$
W_{a}=\frac{\left|E_{a}\right|^{2}}{16 \pi} \frac{\partial\left(\omega \epsilon_{r}\right)}{\partial \omega} .
$$

For a given electrostatic field $E_{a}$ (i.e., a given electronic density perturbation), the rate of energy lost through $I$ andan damping for the individual modes can be compared

$$
\frac{\left(\nu_{\text {slow }} W_{a}^{\text {slow }}\right)}{\left(\nu_{\text {fast }} W_{a}^{\text {fast }}\right)} \approx \frac{\nu_{\text {slow }}}{\nu_{\text {fast }}}\left(1+\frac{\operatorname{Re}\left(\chi_{B e}^{\text {slow }}\right)}{\operatorname{Re}\left(\chi_{e}\right)}\right)=30,
$$

where $\nu_{\text {slow(fast) }}$ is the normalized Landau damping for the slow(fast) mode. Hence, in addition to having a larger Landau damping, the slow mode, in comparison to the fast mode, needs more laser energy to be sustained at a given amplitude.

\section{B. Ion temperature}

Figure 8 compares two measured spectra, one without the interaction beam and the other with an interaction beam intensity of $I=7 \times 10^{15} \mathrm{~W} \mathrm{~cm}^{-2}$. The variation in the amplitude of the thermal peaks is a direct indication of a change in ion temperature. Figure 9 shows the ion temperature increase as a function of the SBS excited ion-acoustic wave amplitude. When the energy in the interaction beam is increased, the ion-wave amplitude increases and the ion temperature rises; we found no change in the electron temperature. The ion temperature was normalized to the electron temperature 


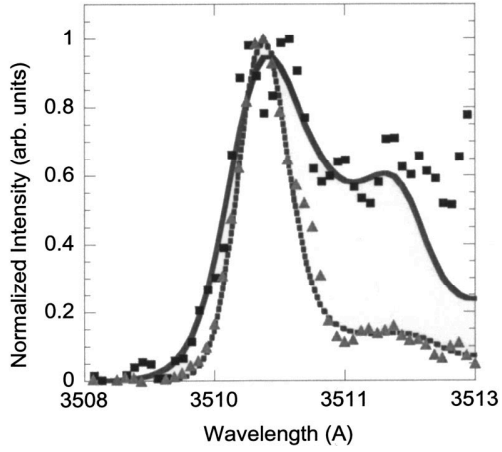

FIG. 8. The blueshifted (thermal) part of the Thomson-scattering spectra for a $10 \%$ Au mixture are shown for a shot without the interaction beam (triangles) and a shot with an interaction beam intensity of $7 \times 10^{15} \mathrm{~W} \mathrm{~cm}^{-2}$ (squares) resulting in an increase in ion temperature from 250 to $475 \mathrm{eV}$.

in Fig. 9 to remove variations in the plasma parameters due to the heater beam. The electron temperature varied by less than $10 \%$ for a given heater beam energy and was independent of the interaction beam intensity. This direct observation of an increase in ion temperature indicates that energy is directly transferred from the SBS driven ion-acoustic waves into the ions. Trapping of Be ions is a plausible mechanism for such an energy transfer.

The SBS instability excites an ion-acoustic wave propagating in the same direction as the interaction beam; when this wave is driven to large amplitude it traps Be ions, accelerating them in its direction of propagation. After thermalization by collisions, the average ion temperature increases.

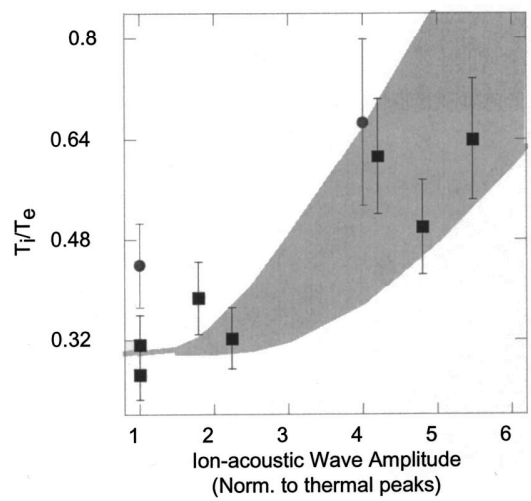

FIG. 9. The ratio of $T_{i} / T_{e}$ as a function of the normalized ion-wave amplitude shows evidence of the generation of hot ions and ion trapping. The ion-wave amplitude is normalized to the thermal ion-wave amplitude. Data for Be plasmas with $5 \% \mathrm{Au}$ (circles) and $10 \% \mathrm{Au}$ (squares) show a factor of two increase in ion temperature The gray area represents calculated resuls from a simple energy balnce between energy deposition due to SBS, and a free-streaming heat flux (Sec. V).

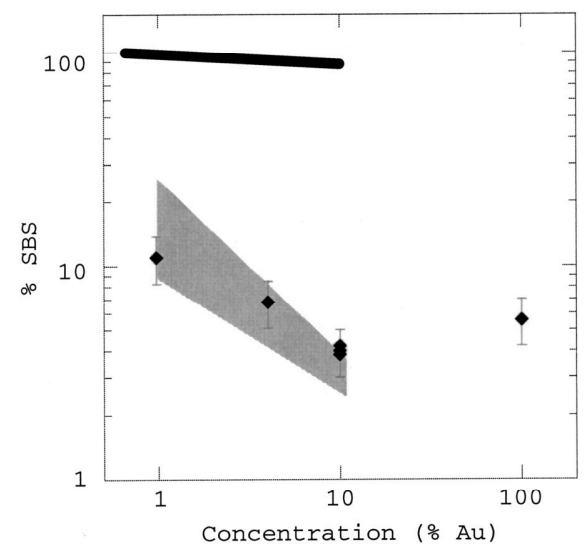

FIG. 10. The SBS reflectivity has been measured to decrease for beryllium plasmas with increasing gold concentration (diamonds). This trend is reproduced by our non-linear SBS theory (shaded area). Linear calculations are also shown (black line).

We measure this increase in ion temperature from the light scattered off thermal ion-acoustic waves counter-propagating to the original hot ions.

\section{SBS reflectivity}

Figure 10 shows the measured SBS reflectivity for the various mixtures of $\mathrm{Au}$ and $\mathrm{Be}$. We observe the SBS reflectivity to be a few percent, while linear theory (i.e., convective gain) predicts a reflectivity of nearly $100 \%$. It is, therefore, clear that while our linear theory allows one to predict which ion-acoustic waves will be driven to large amplitudes by SBS, the SBS reflectivity data (Fig. 10) indicate a need for a nonlinear model that includes saturation of ion-acoustic waves. It should be noted that even after the ion temperature has increased, the fast mode is still weakly damped (Fig. 6) and linear reflectivity predictions are close to $100 \%$, so in these experiments, saturation of the SBS can not be explained by an increase in Landau damping due to global heating of the ions. ${ }^{21}$

\section{MODELING: SATURATION OF THE SBS REFLECTIVITY BY NONLINEAR FREQUENCY SHIFT}

In our range of experimental parameters $\left(6<\mathrm{Z}_{\mathrm{Be}} T_{e} / T_{i}\right.$ $<14$ ) the population of Be ions near the phase velocity of the acoustic wave is large enough so that trapping effects are a plausible saturation mechanism. Trapping suppresses the ionic part of the linear Landau damping of the driven wave by flattening the distribution function around the phase velocity of the wave, ${ }^{7,22}$ therefore, a lower limit for the residual damping, $\nu$, is the electronic Landau damping and a small collisional damping $\left(\nu / \omega_{s} \approx 0.015\right)$. 
Another effect of the trapping is a modification of the dispersion relation for the SBS acoustic wave, creating a nonlinear frequency shift, $\delta \Omega:^{7,23,24}$

$$
\begin{aligned}
\frac{\delta \Omega}{\omega_{a}} \approx & -\eta\left(\frac{\delta n}{n_{e}}\right)^{1 / 2} \\
\equiv & -\frac{f_{B e}}{\sqrt{2 \pi}}\left(v^{4}-v^{2}\right) \\
& \times e^{-v^{2} / 2} \sqrt{7^{B e} \frac{T_{e}}{T_{i}}}\left(\frac{\delta n}{n_{e}}\right)^{1 / 2},
\end{aligned}
$$

where $\eta$ is the detuning parameter, $\omega_{a}$ is the SBS acoustic wave frequency, $f_{\mathrm{Be}}$ is the fraction of Be ions in the plasma, and $v=v_{\phi} / v_{\mathrm{Be}}, v_{\mathrm{Be}}=\sqrt{T_{i} / M_{\mathrm{Be}}}$ is the thermal velocity of the Be ions. The phase velocity $\left(v_{\phi}\right)$ of the fast mode is given by the multi-ion-species dispersion relation $\left(v^{2}\right.$ is of order of $Z_{\mathrm{Be}} T_{e} / T_{i}$ ).

This nonlinear frequency shift detunes the resonant coupling between the interaction beam and the ion wave, therefore saturating the SBS instability. As the ion-acoustic waves are excited to large amplitude, ions are accelerated in the waves potential wells, therefore, slowing the waves phase velocity detuning the SBS resonance so that energy is no longer coupled into these ion-acoustic waves by the incident laser.

Including these effects in a steady-state coupled-wave model of SBS gives ${ }^{25}$

$$
\begin{aligned}
& c \sqrt{1-\frac{n_{e}}{n_{c}}} \partial_{z} A_{1}(z)=-\frac{i \omega_{0}}{4} \frac{\delta n(z)^{*}}{n_{e}} \frac{n_{e}}{n_{c}} A_{0}, \\
& \left(i \eta \sqrt{\frac{\delta n}{n_{e}}}+\nu\right) \frac{\delta n(z)}{n_{e}}=\frac{i \omega_{0}^{2}}{\omega_{a}} \frac{Z_{B e} m_{e}}{m_{i}}\left(\frac{v_{0}}{c}\right)^{2} \frac{A_{1}(z)^{*}}{A_{0}},
\end{aligned}
$$

where $A_{0}\left(A_{1}\right)$ is the incoming (reflected) laser light vector potentials, $\omega_{0}$ is the laser frequency, $v_{0}$ is the electron quiver velocity. Balancing the two terms on the LHS of Eq. (9) shows that detuning becomes important for SBS saturation when $\delta n / n_{e} \approx \nu^{2} / \eta^{2} \simeq 0.1 \%$ for our parameters. Solving the system of equations [Eqs. (8) and (9)] between $z=0$ and $z$ $=L$ leads to

$$
G_{\mathrm{SBS}}(L)=\left\lfloor\ln \left|\frac{\delta n}{n_{e}}\right|+\frac{3 \eta^{2}}{2 \nu^{2}}\left|\frac{\delta n}{n_{e}}\right|\right\rfloor_{0}^{L},
$$

where $G_{\mathrm{SBS}}$ is the usual linear convective gain calculated with the reduced damping discussed above. The logarithmic part is responsible for an exponential growth until the detuning dominates the damping, leading to a polynomial growth in length. The SBS reflectivity for different fractions of $A u$ is shown in Fig. 10 and is in rough agreement with the experimental values. Parameters used in the model were selected to match the experiment $\left(L=800 \mu \mathrm{m}, T_{i} / T_{e} \approx 1 / 2, I_{0}=3\right.$ $\left.\times 10^{15} \mathrm{~W} \mathrm{~cm}^{-2}, n_{e}=10^{20} \mathrm{~cm}^{-3}\right)$. This model is dependent on the electron density and the interaction beam intensity;

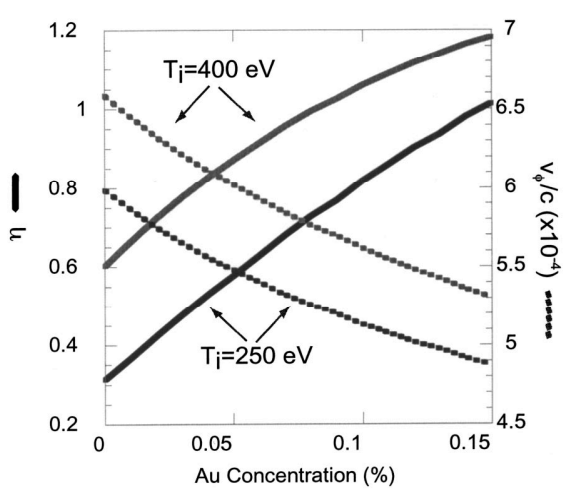

FIG. 11. As more Au ions are added to a Be plasma, the phase velocity of the SBS driven wave decreases (right axis, dashed lines); more Be ions can be trapped, and the detuning parameter $(\eta)$ increases (left axis, solid lines). Two sets of curves are shown for $T_{i}=400$ (upper curves) and $T_{i}=250$ (lower curves).

therefore, the crror bars in Fig. 10 result from uncertaintics in these experimental parameters. The scaling with the fraction of Au ions is well reproduced and is mainly due to a decreasing phase velocity of the fast mode when the fraction of $\mathrm{Au}$ ions is increased which increases the number of ions at the phase velocity. This results in a larger detuning parameter $(\eta)$, and therefore, more efficient detuning (i.e., the same acoustic wave amplitude creates a larger frequency shift) (Fig. 11). It should be noted that for a concentration of $\mathrm{Au}$ greater than $10 \%$, absorption by inverse bremsstrahlung and collisional effects need to be included.

We further test this model by estimating the ion temperature in the Thomson-scattering volume. We balance the energy flux deposited into the acoustic waves (from the Manley-Rowe relations) with a free streaming heat flux: ${ }^{26}$

$$
\frac{\omega_{s}}{\omega_{0}}\left(R_{\mathrm{SBS}} I_{0}\right)(z=L / 2)=n_{B e} T_{i}(z=L / 2) v_{B e}(z=L / 2) .
$$

This model assumes that the energy deposition is local, i.e., the mean-free path of the hot ions accelerated by trapping (a few tens of $\mu \mathrm{m}$ ) is much smaller than the length of the plasma $(L \approx 1 \mathrm{~mm})$, and thermalization is fast enough so heat is carried away by Maxwellian ions when a steady-state is reached. Theoretical and experimental ion-wave amplitudes were related by matching a high reflectivity point; the model then reproduces the experimental scaling for ion temperature. This agreement shows that our modeling of SBS gives a correct estimate of the acoustic wave amplitude in the Thomson-scattering volume, but does not prove that trapping actually occurs; however, trapping is the most plausible means for energy transfer form the waves to the ions with these plasma conditions. The fact that the electron temperature does not increase rules out inverse bremsstrahlung and other energy transfer through electrons. 

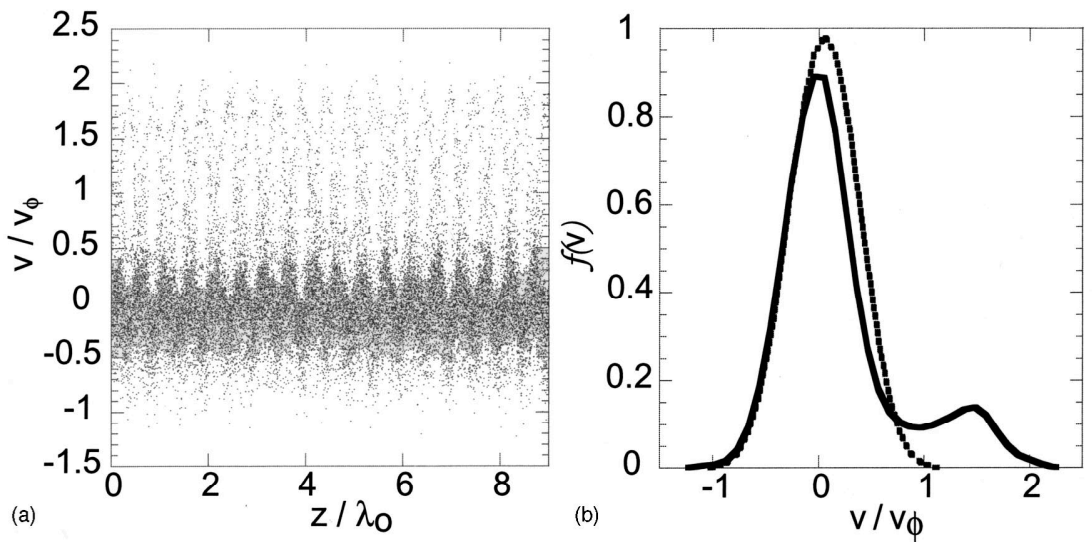

FIG. 12. (a) The results of a PIC simulation of SBS show a phase diagram where trapping can clearly be seen to repeat at the spatial frequency of the driven ion-acoustic wave (b) The Be distribution function evolves from an initial Maxwellian (dashed line), and after $60 \mathrm{ps}$ (solid line) shows a plateau at the phase velocity and a tail of hot ions.

Validation of the main assumptions of this modeling (a steady-state is reached and the nonlinear frequency shift is the main effect that detunes and saturates the instability) through comparisons of particle-in-cell (PIC) and fluid simulations is beyond the scope of this paper, but such a behavior has been emphasized in various publications $s^{3,27,28}$ and preliminary PIC simulations of SBS, with the experimental parameters, using the hybrid code Bzohar ${ }^{7}$ show trapping of the Be ions by the SBS acoustic wave. Figure 12 shows the distribution of particles in phase space where trapped particles follow closed orbits and concurrently the distribution function exhibits a hot tail with ions moving up to twice the phase velocity. The saturation mechanism governed by detuning will be much weaker in high Z-plasmas $\left(\mathrm{CO}_{2}\right.$, pure $\mathrm{Au}$ ) because the detuning parameter is a strong function of charge state.

\section{SUMMARY}

In summary, we have presented measurements of the growth of ion-acoustic waves in well-characterized multiion-species plasmas. Our measurements of the SBS reflectivity indicate the need for a saturation mechanism. Using Thomson scattering and adding a small fraction of Au ions to a Be plasma enabled an accurate measurement of $T_{i}$, while $T_{i}$ was found to increase with SBS reflectivity and with the amplitude of the (local) acoustic waves, $T_{e}$ was found to be constant. This is consistent with our model where the increase in $T_{i}$ is due to trapping of ions by the SBS ionacoustic waves, while the electron-ion collision rate and the electronic part of the Landau damping is very small, so that $T_{e}$ and $T_{i}$ are de-coupled.

\section{ACKNOWLEDGMENTS}

We would like to acknowledge the efforts of the Trident laser crew: T. Hurry, R. Gonzales, N. Okamoto, F. Archuleta,
S. Letzring, and R. Perea. We thank R. Scalettar, J. Fernandez, and A. Hauer, and the UC Davis Physics Department for their support.

This work is partially supported by LDRD 01-ERD-107 and was performed under the auspices of the U.S. Department of Energy by the Lawrence Livermore National Laboratory under Contract No. W-7405-ENG-48.

${ }^{1}$ H. A. Rose and D. F. DuBois, Phys. Rev. Lett. 72, 2883 (1994) ${ }^{2}$ R. L. Berger, C. Still, E. A. Williams, and A. B. Langdon, Phys. Plasmas 5, 4337 (1998).

${ }^{3}$ C. E. Clayton, C. Joshi, and F. F. Chen, Phys. Rev. Lett. 51, 1656 (1983).

${ }^{4}$ S. II. Glenzer, L. M. Divol, R. L. Berger, C. Geddes, R. K. Kirkwood, J.

Moody, E. A. Williams, and P. E. Young, Phys. Rev. Lett. 86, 2565 (2001).

${ }^{5}$ B. Gellert and B. Kronast, Appl. Phys. B: Photophys. Laser Chem. 32, 175 (1983).

${ }^{6}$ B. Gellert and B. Kronast, Appl. Phys. B: Photophys. Laser Chem. 33, 29 (1984).

${ }^{7}$ B. I. Cohen, B. F. Lasinski, A. B. Langdon, and E. A. Williams, Phys. Plasmas 4, 956 (1997)

${ }^{8}$ N. K. Moncur, R. P. Johnson, R. G. Watt, and R. B. Gibson, Appl. Opt. 34, 4274 (1995).

${ }^{9}$ G. B. Zimmerman and W. L. Kruer, Comments Plasma Phys. Controlled Fusion 2, 51 (1975).

${ }^{10}$ L. J. Suter, A. A. Hauer, L. V. Powers, D. B. Ress, N. Delameter, W. W. Hsing, O. L. Landen, A. R. Thiessen, and R. E. Turner, Phys. Rev. Lett. 73, 2328 (1994)

${ }^{11}$ L. J. Suter, R. L. Kauffman, C. B. Darrow, A. A. Hauer, H. N. Kronblum, O. L. Landen, T. J. Orzechowski, D. W. Phillion, J. L. Porter, L. V. Powers et al., Phys. Plasmas 3, 2057 (1996).

${ }^{12} \mathrm{~S}$. H. Glenzer, W. Rozmus, B. J. MacGowan, K. G. Estabrook, J. S. D. Groot, and G. B. Zimmerman, Phys. Rev. Lett. 82, 97 (1999).

${ }^{13}$ B. S. Baner, R. P. Drake, K. G. Fstahrook, R. G. Watt, M Wilke, and S. A Baker, Phys. Rev. Lett. 74, 3604 (1995)

${ }^{14}$ D. S. Montgomery, R. P. Johnson, H. A. Rose, J. A. Cobble, and J. C. Fernandez, Phys. Rev. Lett. 84, 678 (2000).

${ }^{15}$ B. Bai, J. Zheng, W. Liu, and C. X. Yu, Phys. Plasmas 8, 4144 (2001).

${ }^{16}$ E. A. Williams, R. L. Berger, R. P. Drake, A. M. Rubenchik, B. S. Bauer. D. D. Meyerhofer, A. C. Gaeris, and R. P. Johnson, Phys. Plasmas 2, 129 (1995).

${ }^{17}$ B. Bezzerides, H. X. Vu, and J. M. Wallace, Phys. Plasmas 3, 1073 (1995). ${ }^{18}$ J. A. Fejer, Can. J. Chem. 38, 1114 (1960). 
${ }^{19}$ D. E. Evans, Phys. Plasmas 12, 573 (1970).

${ }^{20}$ L. V. Powers, R. L. Berger, R. L. Kauffman, B. J. MacGowan, P. A. Amendt, C. A. Back, T. P. Bernat, S. N. Dixit, D. I. Eimerl, K. G. Es-

tabrook et al., Phys. Plasmas 2, 2473 (1995).
${ }^{21}$ P. W. Rambo, S. C. Wilks, and W. L. Kruer, Phys. Rev. Lett. 79, 83 (1997).

${ }^{21}$ P. W. Rambo, S. C. Wilks, and W. L. Kruer, Phys. Rev. Let. 75

${ }^{22}$ V. E. Zakharov and V. I. Karpman, JETP 16, 351 (1962).
${ }^{23}$ A. A. Andreev and V. T. Tikhonchuk, JETP 68, 1135 (1989).

${ }^{24}$ G. J. Morales and T. M. O'Neil, Phys. Rev. Lett. 28, 417 (1972)
${ }^{25}$ E. A. Williams and B. I. Cohen, Bull. Am. Phys. Soc. 46, 284 (2001).

${ }^{26}$ W. L. Kruer, Phys. Fluids 23, 1273 (1980).

${ }^{27}$ S. C. Wilks, W. L. Kruer, J. Denavit, K. G. Estabrook, D. E. Hinkel, D. Kalantar, A. B. Langdon, B. MacGowan, D. S. Montgomery, and E. A. Williams, Phys. Rev. Lett. 74, 5048 (1995)

${ }^{28} \mathrm{H}$. X. Vu, D. F. DuBois, and B. Bezzerides, Phys. Rev. Lett. 86, 4306 (2001). 
Appendix C

"Stimulated Brillouin scattering

(SBS) in the saturated regime", D.

H. Froula, et al., Physics of

Plasmas Special Issue, Accepted

for publication May (2003)

(APS-DPP Invited) 


\title{
Stimulated Brillouin scattering in the saturated regime
}

\author{
D. H. Froula* \\ Physics Department, University of California, Davis, 95616
}

L. Divol, D. G. Braun, B. I. Cohen, G. Gregori, A. Mackinnon, E. A. Williams, and S. H. Glenzer Lawrence Livermore National Laboratory, University of California, P.O. Box 808, Livermore, California 94551

H. A. Baldis

Department of Applied Science. University of California at Davis, Davis, 95616 and Physics and Advanced Technologies, University of California, P.O. Box 808, Livermore, CA 94551

D. S. Montgomery and R. P. Johnson Los Alamos National Laboratory, Los Alamos, New Mexico 87545

(Date textdate; Received textdate; Revised textdate; Accepted textdate; Published textdate)

An experimental study of the stimulated Brillouin scattering (SBS) instability has investigated the effects of velocity gradients and kinetic effects on the saturation of ion-acoustic waves in a plasma. For intensities less than $\mathrm{I}<1.5 \times 10^{15} \mathrm{~W} \mathrm{~cm}{ }^{3}$, SBS is in a linear regime and is moderated primarily by velocity gradients, while for intensities above this threshold, non-linear trapping is relevant. We report direct evidence of detuning of SBS by a velocity gradient which was achicved by directly measuring the frequency of the SBS-driven acoustic wave relative to the local resonant acoustic frequency. The frequency and amplitude of the ion-acoustic wave directly responsible for SBS has been measured as a function of space using a $3 \omega 200 \mathrm{ps}$ Thomson-scattering probe beam. Furthermore, a novel use of Thomson scattering has allowed us to gather direct evidence of kinetic effects associated with the SBS process in the non-linear regime. Specifically, a measured two-fold increase in the ion temperature has been linked with ion-acoustic waves that have been driven to large amplitudes by the SBS instability. Ion-acoustic waves were excited to large amplitude with a $2 \omega 1.2$-ns long interaction beam with intensities up to $7 \times 10^{15} \mathrm{~W} \mathrm{~cm}^{-2}$. The measured two-fold increase in the ion temperature and its correlation with SBS reflectivity measurements provides quantitative evidence of hot ions created by ion trapping in laser plasmas. These detailed and accurate measurements in well-characterized plasma conditions allow a direct test of linear and non-linear models of the saturation of SBS.

PACS numbers: $52.25 . \mathrm{Qt}, 52.35 . \mathrm{Fp}, 52.40 . \mathrm{Nk}, 52.50 . \mathrm{Jm}$

\section{INTRODUCTION}

An understanding of the propagation of laser light into an underdense plasma is critical in the development of indirect-drive inertial confinement fusion. Understanding ion-wave growth and the saturation mechanisms in these plasmas is necessary to improve the energy coupling into the fusion capsule. Currently models are being developed to predict stimulated Brillouin scattering (SBS laser losses in high temperature, large-scalelength plasmas that will be achieved in inertial confinement fusion at future facilities such as the National Ignition Facility $[1,2]$. We will present measurements that have verified both hydrodynamic (velocity gradients) and kinetic (trapping) effects on SBS.

The SBS instability results from the resonant coupling of an intense laser pulse, a scattered light wave, and an ion-acoustic wave. SBS excites an ion-acoustic wave from

*Lawrence Livermore National Laboratory, University of California, P.O. Box 808, Livermore, California 94551 thermal fluctuations to large amplitude where it has been shown to saturate [3-6]. Previous models have suggested non-linear saturation mechanisms such as two-ion-decay, trapping, and wave breaking $[7-10]$. Theory has long predicted that velocity gradients will create a mismatch in the parametric SBS instability therefore, reducing the growth of ion-acoustic waves [11]. Detuning of SBS can be a powerful mechanism for limiting the growth of ionacoustic waves because the instability relies on a resonant three wave process. Any shift in the local frequency of the ion-acoustic resonance relative to the ponderomotive frequency, created by the incident and reflected light, will limit the growth of the SBS instability.

Experiments at the Trident Laser Facility observe detuning of the SBS instability by a velocity gradient and by kinetic effects. Direct evidence of detuning of SBS by a velocity gradient was observed through a novel use of two Thomson-scattering diagnostics allowing us to measure the frequency and amplitude of the ion-acoustic waves directly responsible for SBS. By comparing the local ion-acoustic frequency with the frequency of the driven acoustic wave, we have measured the actual detuning of the SBS instability. 
We will show that ion-acoustic waves and the SBS reflectivity saturate for intensities above $\mathrm{I}>1.5 \times$ $10^{15} \mathrm{~W} \mathrm{~cm}{ }^{-2}$ where we have seen direct effects of trapping. Adding a small amount of Au to our Be plasmas allowed the accurate determination of the ion temperature by measuring the relative damping of two ion-acoustic modes. We report up to a factor of two increase in ion temperature when ion-acoustic waves are excited to large amplitude by SBS [12]. This increase in ion temperature is a strong indication of hot ions due to trapping.

In Sec. II of this paper we present the experimental setup. Section III describes the measurements made to characterize the plasma while Sec. IV discusses SBS saturation experiments and presents observed linear and non-linear saturation mechanisms. Section VI summarizes our findings.

\section{EXPERIMENTAL SETUP}

\section{A. Beam and Target configuration}

T'he experiments used a three-laser beam configuration at the Trident Laser Facility [13]. The plasmas were produced by a heater beam with $180 \mathrm{~J}$ of $2 \omega(\lambda=527 \mathrm{~nm})$ laser light in a 1.2-ns-long square pulse. The heater beam was focused normal to the target surface (Fig. 1a) using an $f / 6$ lens and a strip-line random phase plate. This produced a $1200 \mu \mathrm{m} \times 100 \mu \mathrm{m}$ line focus with an intensity of $10^{14} \mathrm{~W} \mathrm{~cm}^{-2}$ [14]. The targets where cut to vary the length of the plasma along the interaction beam axis between $L=1000 \mu \mathrm{m}$ and $L=250 \mu \mathrm{m}$. A $2 \omega$ interaction beam with a maximum energy of $230 \mathrm{~J}$ in a 1.2 ns-long square pulse was aligned parallel to the target surface (Fig. 1c). The interaction heam was focused to $\mathrm{a} \sim 60 \mu \mathrm{m}$ diameter spot, resulting in intensities up to $7 \times 10^{15} \mathrm{~W} \mathrm{~cm}^{-2}$. The interaction beam was used to drive SBS which excites ion-acoustic waves $\left(\mathbf{k}_{\|}=\mathbf{2} \mathbf{k}_{\mathbf{2}}\right)$ in the plasma co-propagating in the direction of the interaction beam (Fig. 1b).

The third laser beam, $3 \omega(\lambda=3513.0 \AA)$, was aligned $48^{\circ}$ from the interaction beam and was used as a Thomson-scattering probe beam (Fig. 1a). The probe beam was focused to a $50 \mu \mathrm{m}$ spot overlapping the focus of the interaction beam. The probe beam and Thomson-scattering collection optics define a volume $(70 \mu \mathrm{m} \times 70 \mu \mathrm{m} \times 60 \mu \mathrm{m})$ located at the center of the target chamber. The $2 \omega$ interaction beam was timed to turn on $1.0 \mathrm{~ns}$ after the heater beam. The 180 ps Gaussian $3 w$ probe bean was turned on 400 ps after the interaction beam near the peak of the backscattered SBS light.

\section{B. Thomson scattering}

Thomson scattering provides a measure of the frequencies and amplitudes of co- and counter-propagating ionacoustic waves in a plasma. From the measured resonant

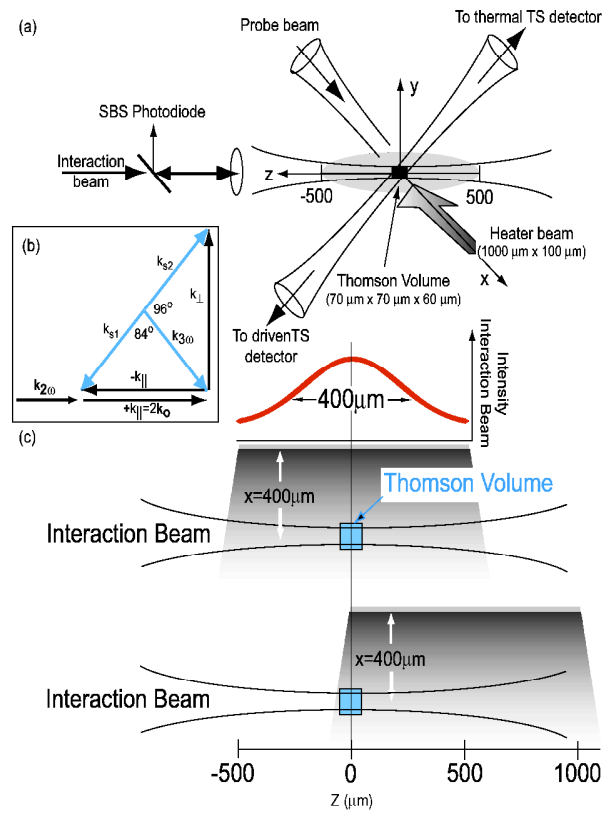

FIG. 1: (a) The experimental setup is shown with three beams intersecting the Thomson volume located at target chamber center. Light is collected by two f/5 collection lenses. (b) A diagram detailing the ion-acoustic wave-vectors probed is shown. (c) The target was moved to spatially probe ionacoustic waves in the plasma; the location of the Thomson volume and the focus of the interaction beam remain at target chamber center.

frequencies, the local sound speed, electron temperature, and particle flow can be determined. The amplitude of the ion-acoustic wave is proportional to the square root of the measured intensity. In multi-ion-species plasmas, the relative amplitude of the light scattered from ion-acoustic waves allows an accurate determination of the ion temperature $[15-17]$. In theory, the ion temperature can also be obtained from the width of the intensity peaks in the Thomson-scattering spectra, but velocity and temperature gradients within the Thomson-scattering volume make this measurement uncertain and therefore, unreliable in laser-produced plasmas.

In our experiment, the wavelength of the probe laser, the scattering geometry, and the plasma parameters result in collective Thomson scattering from fluctuations characterized by wave numbers such that the scattering parameter is greater than one, $\alpha=1 / \mathrm{k}_{a} \lambda_{D} \approx 3$, where $\mathrm{k}_{a}$ is the acoustic scattering wave vector and $\lambda_{\nu}$ is the electron Debye length. The ion-acoustic scattering wave vector is determined by energy and momentum 
equations:

$$
\begin{aligned}
& \mathbf{k}_{\mathbf{a}}=\mathbf{k}_{\mathbf{o}}-\mathbf{k}_{\mathrm{s}} \\
& \omega_{a}=\omega_{0}-\omega_{\mathrm{s}} .
\end{aligned}
$$

For low-frequency fluctuations, $\mathrm{k}_{0} \sim \mathrm{k}_{\mathrm{s}}$ and the acoustic wave vector is given by,

$$
k_{a}=2 k_{o} \sin \left(\frac{\theta}{2}\right)
$$

where $\theta$ is the angle between the Thomson-scattering probe and the collection optics (Fig. 1b).

When observing low frequency collective effects with Thomson scattering, the frequency of the scattered light is shifted from the laser frequency in two ways: by the frequency of the ion-acoustic wave in the frame of the plasma $\left(\omega_{a}=c_{s} k_{a}\right)$, and by the relative ion flow in the laboratory frame $\left(\mathrm{v}_{f}\right)$. The frequency shift due to the ion-acoustic waves is given by,

$$
\Delta \lambda_{\text {iaw }}=\frac{\lambda_{3 \omega}^{2}}{2 \pi c}\left(\mathbf{c}_{s} \mathbf{k}_{w}\right)
$$

while the ion flow shifts the spectral peaks by,

$$
\Delta \lambda_{\text {flow }}=\frac{\lambda_{3 \omega}^{2}}{2 \pi c}\left(\overrightarrow{\mathrm{k}}_{a} \cdot \overrightarrow{\mathrm{v}}_{f}\right)
$$

where the dispersion relation is given by,

$$
\frac{\omega}{k}=c_{s}=\sqrt{\frac{Z k_{b} T_{e}}{m_{i}}+3 \frac{k_{b} T_{i}}{m_{i}}} .
$$

$\mathrm{k}_{b}$ is Boltzmann's constant and $\mathrm{m}_{i}$ is the ion mass and $\mathrm{Z}$ is the particles average charge state. Therefore, if the sound speed is known, the frequency shift of one peak in the Thomson-scattering spectra relative to the frequency of the probe laser provides a measure of the plasma flow.

We employed two separate Thomson-scattering spectrometers observing the same volume and location in the plasma (Fig. 1a). One of the Thomson-scattering diagnostics measured light scattered from the SBS excited ion-acoustic waves ( $\mathbf{k}_{a}=\mathbf{k}$ see Fig. 1b) which propagate parallel to the interaction beam (Fig. 2a). The other Thomson-scattering diagnostic probes ion-acoustic waves propagating perpendicular $\left(\mathrm{k}_{\alpha}=\mathrm{k} \perp\right.$ see $\mathrm{Fig}$. 1b) to the interaction beam (Fig. 2b). The latter allows us to measure the local sound speed (proportional to the square root of the electron temperature), while the former measures both the frequency of the excited ion-acoustic wave (from $\mathbf{k}_{\mid}$co-propagating in the direction of the interaction beam) and the plasma flow along the interaction beam (-k counter-propagating in the direction of the interaction beam).

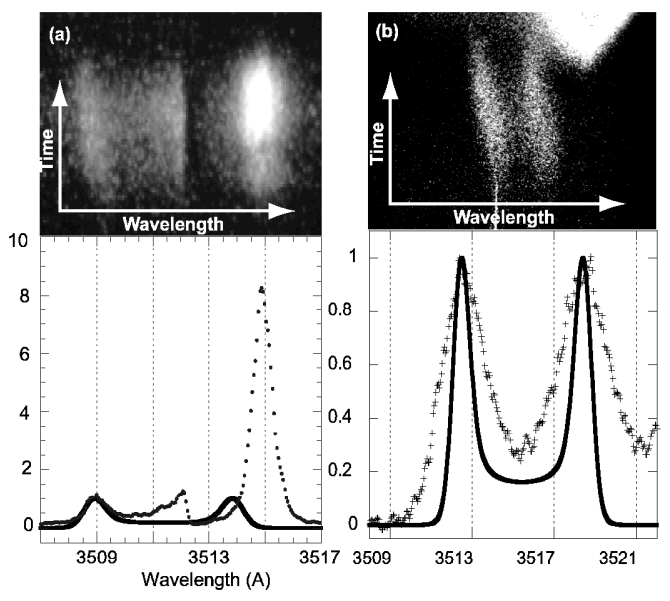

FIG. 2: (a) The driven Thomson scattering spectra provides a measure of both the plasma flow along the axis of the interaction beam $\left(-\mathbf{k}_{||}\right)$, the frequency of the SBS driven acoustic wave $\left(\mathbf{k}_{\|}\right)$, and the relative amplitude of the SBS driven ion-

acoustic waves. This data is representative of a point at $\mathrm{z}$-$375 \mu \mathrm{m}$ and $\mathrm{x}=400 \mu \mathrm{m}$ with an interaction beam intensity of $\mathrm{T}=1.5 \times 10^{15} \mathrm{~W} \mathrm{~cm}{ }^{-2}$. (h) Our thermal Thomson-scattering spectra allow us to measure the electron temperature by observing ion-acoustic waves propagating perpendicular $\left(k_{\perp}\right)$ to the driven ion-acoustic waves $\left(\mathrm{k}_{\mid}\right)$.

\section{Full aperture backscatter station}

Light incident on a plasma can couple into electron and ion waves driving instabilities which can backscatter large amounts of energy [6]. The stimulated Brillouin (SBS) and stimulated Raman (SRS) instabilities result from the resonant coupling of the incident and scattered electromagnetic waves with an ion-acoustic wave (in the case of SBS) and an electron plasma wave (in the case of SRS).

The backscattered light was collected and collimated by the focusing lens of the interaction beam (Fig. 1a). Four percent of the backscattered light was split onto a diagnostics table (Fig. 3) where a fast photodiode measured the absolute SBS reflectivity. To measure the SBS and SRS spectra (see Sec. III C), the reflections from the front and back surface of a wedge placed before the photodiode further split the backscattered light into $400 \mu \mathrm{m}$ fiber optic cables coupled to 1 -meter and 1/4-meter spectrometers. The fibers where placed at the focus of the FABS focusing lens. The light was spatially smoothed by two $3^{\circ}$ diffusers $5 \mathrm{~cm}$ from the fiber optic entrances. The diffusers expanded the spot on the fiber making alignment less critical. The fibers were mounted at the slits of a 1 meter SBS and $1 / 4$ meter SRS spectrometer. The 


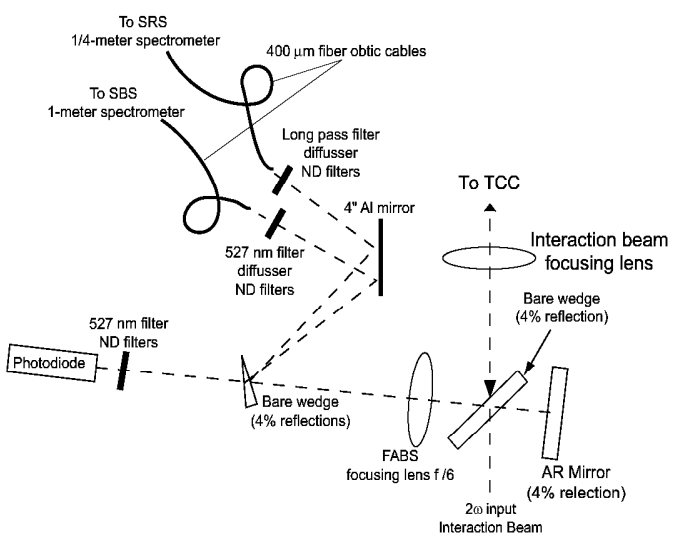

FIG. 3: Light backscattered from the plasma is collected and collimated by the focusing lens of the interaction beam. The light is split by a $4 \%$ wedge to the diagnostic table wher the SBS and SRS spectrum were measured. A photodiode measured the absolute SBS reflectivity.

SBS spectrometer used a 1200 lines $/ \mathrm{mm}$ grating and a $200 \mu \mathrm{m}$ slit to obtain a spectral resolution of $0.05 \mathrm{~nm}$ The SRS spectrometer used a 150 lines $/ \mathrm{mm}$ grating with a $400 \mu \mathrm{m}$ slit to obtain a resolution of $0.5 \mathrm{~nm}$. A longpass glass optical filter (OG570) was used between the output of the SRS spectrometer and the streak camera to filter stray light below $570 \mathrm{~nm}$. In both cases, a tempora resolution of 100 ps was achieved using two Hamamatsu streak cameras.

A four-percent reflection from the interaction beam has been used to provide a shot-to-shot calibration for the photodiode. This beam was split by a wedge along the path of the interaction beam before the focusing lens (Fig. 3). The split energy is reflected by an AR mirror where it is directed back through the wedge and focused onto the photodiode. This reference pulse arrives at the photodiode 15 ns after the light from target chamber center. The data was reduced by numerically integrating each pulse; the SBS reflectivity is the ratio of the integrated signals adjusted for the energy loss $(96 \%)$ at the AR mirror.

\section{PLASMA CHARACTERIZATION}

\section{A. Plasma temperature}

The plasma has been well-characterized [18]. Figure 4 shows a constant electron temperature profile $\left(\mathrm{T}_{e}=\right.$ $450 \mathrm{eV}$ ) along the interaction beam axis. Figure $2 \mathrm{~b}$ shows a typical Thomson-scattering spectrum $\left(\mathrm{k}_{\perp}\right)$. Two peaks corresponding to thermal ion acoustic fluctuations prop

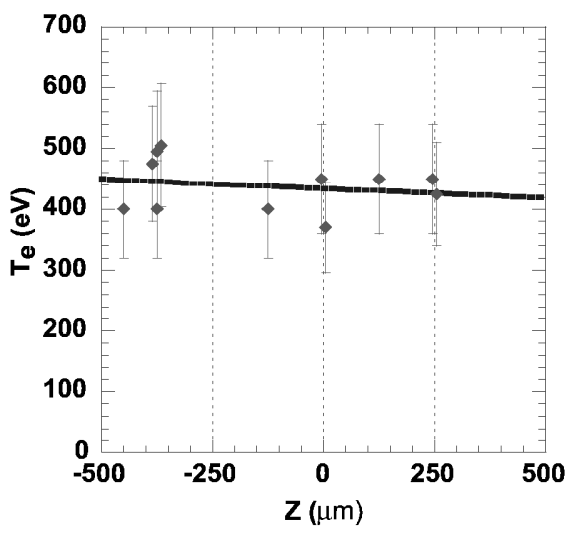

FIG. 4: The electron temperature was measured to be a constant $\mathrm{T}_{e}=450 \mathrm{eV}$ along the interaction beam axis, $\mathrm{x}=400$ $\mu \mathrm{m}$ from the surface of the target. The line is a best fit.

agating perpendicular to the interaction beam $\left(\mathrm{k}_{\perp}\right)$ are evident. The data are fit using a standard theoretical form factor which uses the electron temperature as the only sensitive free parameter for our fully ionized Be plasmas $[19]$. The measured width of the peaks $(\delta \lambda)$ gives an estimated velocity range $\frac{\delta v}{\alpha}\left(\propto \frac{\delta \lambda}{\lambda}\right)=0.5$ within the Thomson-scattering volume. This broadening is to be expected as the initial plasma length is only $\mathrm{y}=100 \mu \mathrm{m}$ in the perpendicular direction and therefore, the Thomsonscattering volume is centered around the outward expansion of the plasma. An error of $20 \%$ in the electron temperature is due to the broad Thomson-scattering spectra. The error bars were determined by fitting the form factor over a reasonable spread in the data.

In previous experiments the ion temperature has been measured using Be plasmas with small amounts of $\mathrm{Au}$. Measuring the relative damping of two kinetic modes produced in these multi-ion-species plasmas allowed us to determine the ion temperature with high accuracy. In this study we found the ion temperature to be $T_{i}(\mathrm{x}=$ $300 \mu \mathrm{m})=250 \mathrm{eV}[15]$.

\section{B. Plasma flow}

Figure 2a shows a Thomson-scattering spectra in which the probed ion-acoustic waves are co- and counterpropagating in the dircetion of the interaction bcam $\left(\mathrm{k}_{\|}\right)$. The blue-shifted light is scattered from ion-acoustic fluctuations $\left(-\mathbf{k}_{\mid}\right)$, i.e., the standard Thomson-scattered signal. The form factor is fit to the blue-shifted intensity peak using the independently measured electron temperature and a flow of $\frac{\mathrm{y}}{c_{\mathrm{s}}}=0.7$. The red-shifted intensity peak in the theoretical fit $(\lambda=3513.8 \AA)$ corresponds to 


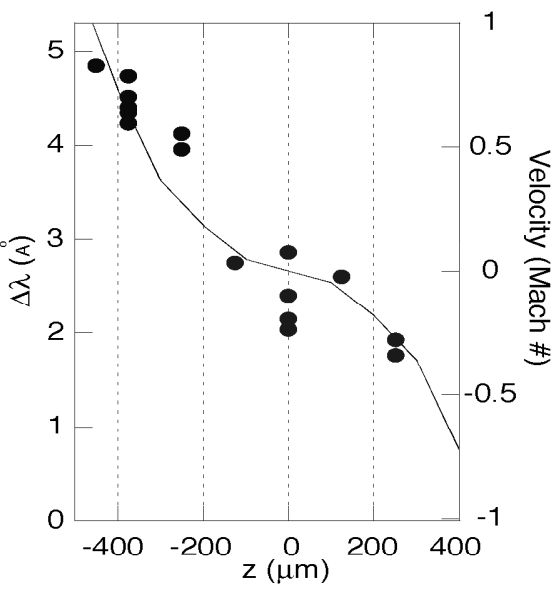

FIG. 5: The frequency shift of the peak intensity scattered from thermal $\left(-\mathbf{k}_{\|}\right)$ion-acoustic waves allows us to measure a velocity profile along the interaction beam axis. A LASNEX simulation using a 1-mm disk target reproduces the velcocity profile.

the local ion-acoustic resonance for waves co-propagating in the direction of the interaction beam $\left(\mathbf{k}_{\mid}\right)$. Note that the use of two Thomson-scattering diagnostics is necessary as the driven acoustic wave is not assumed to be at the local resonant frequency. In fact, from Fig. 2a it is evident that the SBS-driven ion-acoustic waves are of resonance (see Sec. IV A)

By moving the target, we have used scattering from the ion-acoustic fluctuations $\left(-\mathbf{k}_{\mid}\right)$to map out the velocity flow (Fig. 5). A $200 \mu \mathrm{m}$ velocity plateau in the middle of the plasma is evident while a Mach 1 flow was measured in the front of the plasma. A LASNEX [20] simulation using a 1-mm circular target with an average intensity of $10^{14} \mathrm{~W} \mathrm{~cm}^{-2}$ calculated a radial flow $400 \mu \mathrm{m}$ from the target that compares well with our data in Fig. 5.

\section{Plasma electron density}

The frequency of the light backscattered by the electron plasma wave (SRS) is a strong function of the electron plasma frequency $\left(\omega_{p e}^{2}=\frac{n_{e} e^{2}}{\varepsilon_{o} m_{e}}\right.$ ) and therefore, the density [21-24],

$$
\omega=\sqrt{\omega_{p e}^{2}+\frac{3 \mathrm{k}^{2} k_{b} \mathrm{~T}_{e}}{\mathrm{~m}_{e}}}
$$

where $k_{b}$ is Boltzmann's constant, $m_{e}$ is the mass of an electron, and $\mathrm{k}=2 k_{0}$ is the wave vector of the probed
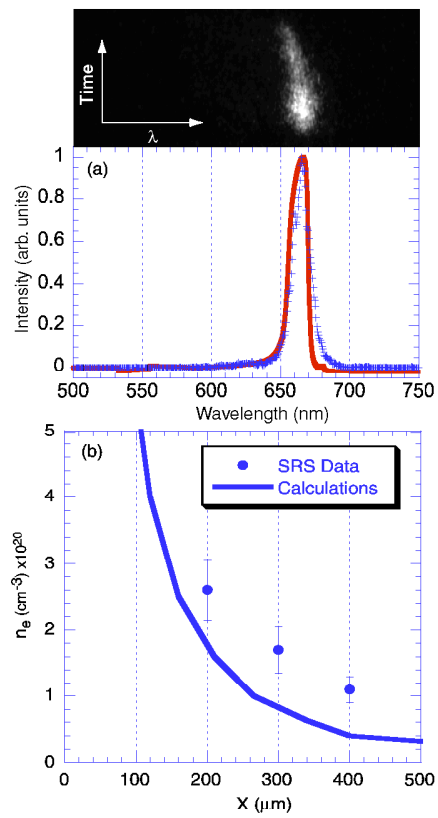

FIG. 6: (a) The SRS spectra is measured at $\mathrm{x}=400 \mu \mathrm{m}$ from the target surface. A normalized linear gain calculation is fit to the data (line) to obtain the density. (b) The electron density calculated from the SRS spectra are plotted as a function of the distance from the target surface. LASNEX calculations are plotted (line).

electron plasma wave.

Figure 6a shows the measured SRS spectrum for a shot where the interaction beam was focused $\mathrm{x}=400 \mu \mathrm{m}$ from the target surface. The spectrum peaks at $665 \mathrm{~nm}$ which corresponds to a density of $n_{e}(x=400 \mu \mathrm{m})=1.2 \times 10^{20}$ $\mathrm{cm}^{-3}$. The spectra was fit using a linear gain calculation. To match the spectral width a $10 \%$ density gradient was assumed over $300 \mu \mathrm{m}$. 'The corresponding SRS gain was $\mathrm{G}_{\text {srs }}=10$. The electron density profile normal to the target surface is shown in Fig. 6b.

\section{SATURATED SBS REGIME}

Figure 2 a shows Thomson-scattering data in which the SBS instability driven by the interaction beam has excited ion-acoustic waves $\left(\mathbf{k}_{\|}\right)$to large amplitude. The intensity of the scattered light is proportional to the square root of the amplitude of the probed ion-acoustic wave $\left(\mathbf{I}^{2} \propto \frac{\delta n}{n_{e}}\right)$. In Fig. 7, the local normalized ionacoustic wave amplitude $(\mathrm{z}=-375)$ and the SBS reflectivity are plotted as a function of the intensity of the 


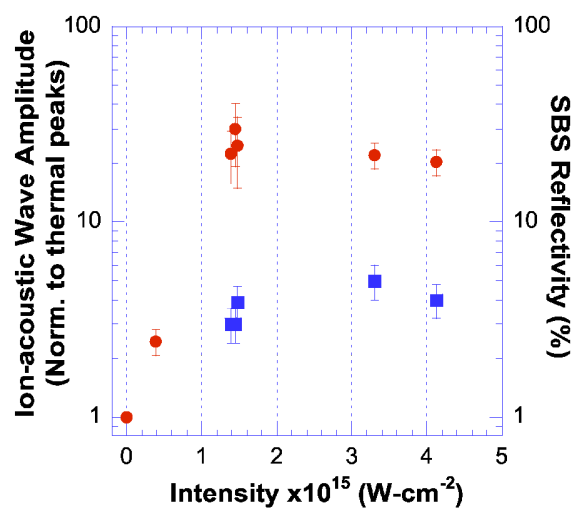

FIG. 7: The ion-acoustic wave amplitude and the SBS reflectivity are plotted as a function of the intensity of the interaction beam. The target was moved to probe ion waves at $\mathrm{z}=-375$. The plasma length along the interaction beam was $\mathrm{L}=1-\mathrm{mm}$.

interaction beam. The amplitude of the local SBS driven ion-acoustic waves and the SBS reflectivity saturate for intensity $\mathrm{I}>1.5 \times 10^{15} \mathrm{~W} \mathrm{~cm}^{-2}$. The intensity was varied by changing the energy from 10 to $150 \mathrm{~J}$ while all other parameters were held fixed.

Various saturation mechanisms have been suggested to explain ion-wave saturation, including frequency detuning induced by trapping $[3,12,25]$, two-ion-wave-decay $[6,26-28]$, increased linear Landau damping due to kinetic ion heating $[29,30]$, nonlinear damping associated with wave-breaking and trapping $[7,8]$, and energy coupling with higher harmonics $[31,32]$. Detuning of SBS can be a powerful mechanism for limiting the growth of ion-acoustic waves because the instability relies on a resonant three-wave process. Any shift in the local frequency of the SBS driven ion-acoustic wave relative to the frequencies of the incident and/or reflected light will limit the growth of the SBS instability. In the following sections we will investigate detuning of the local ion-acoustic wave due to velocity gradients and kinetic effects. We have found that velocity gradients increase the threshold for linear growth, but for intensities above I $\geq 1.5 \times 10^{15}$ $\mathrm{W} \mathrm{cm}^{-2}$ non-linear kinetic effects saturate SBS

\section{A. Velocity gradient}

The measured velocity gradient Doppler shifts the local resonant frequency of the ion-acoustic waves, detuning the SBS resonance, therefore, saturating the driven ion-acoustic wave. From Fig. $2 a$ it is evident that the SBS driven ion-acoustic waves are off resonance. The local resonant frequency for acoustic waves with $\mathbf{k}_{\|}$(shown

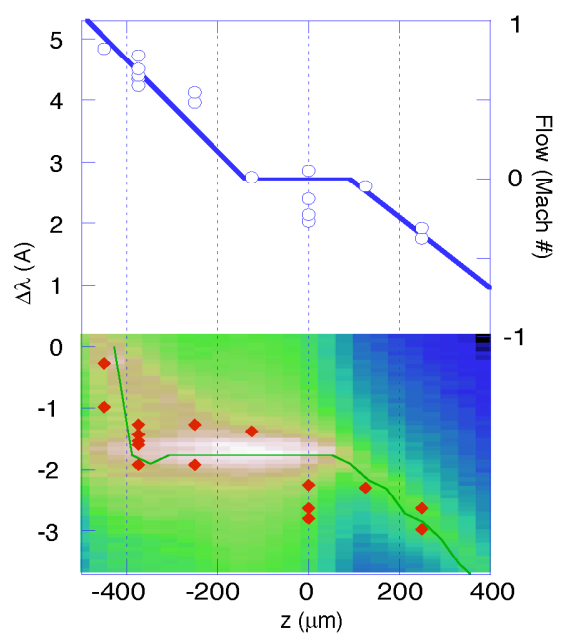

FIG. 8: The wavelength shift of the peak intensity for the down shifted (solid squares) and up-shifted (open circles) Thomson-scattering peaks are plotted. The up-shifted intensity peaks reveal a Mach 1 flow parallel to the interaction beam (right axis). The data are plotted on top of a pF3d simulation where ion-acoustic waves are driven to large amplitude in the front of the velocity plateau.

by the red-shifted intensity peak in the theoretical form factor) is lower than that of the measured SBS driven ion-acoustic wave by $1.1 \AA$, which shows that SBS is detuned by the velocity gradient (i.e. SBS-driven acoustic waves are locally off resonance). Figure 8 shows that the frequency of the driven ion-acoustic wave remains unchanged as one moves away from the velocity plateau and towards the front of the plasma. This indicates that the frequency of the ponderomotive force driving the ionacoustic waves in the front of the plasma is constant

From Fig. 9 it is apparent that SBS grows rapidly in the plateau creating a large scattered light wave. This scattered light wave beats with the incident light wave creating a constant frequency ponderomotive force in the front of the plasma (i.e., the frequency of the light scattered from the plateau and the frequency of the incident light are constant). From the resonant acoustic wave, it is apparent that the acoustic resonance is changing as one move towards the front of the plasma, therefore, the constant ponderomotive frequency created in the plateau is increasingly off resonance (right axis Fig. 8) in the front of the plasma and the spatial amplitude of the ionacoustic waves can not resonantly grow in the front of the plasma.

The experiment was simulated in 2-D using pF3d [1]. A $f / 6$ RPP beam with a best focus of $60 \mu \mathrm{m}$ and an average vacuum intensity of $10^{15} \mathrm{~W} \mathrm{~cm}{ }^{-2}$ was propagated 
through a $\mathrm{L}=1000 \mu \mathrm{m}$ slab of plasma $\left(\mathrm{T}_{e}=450 \mathrm{eV}\right.$, $\left.\mathrm{n}_{e}=1.2 \times 10^{20} \mathrm{~cm}^{-3}\right)$. A Mach 2 transverse flow and the measured velocity profile along the propagation axis were included (right axis Fig. 8)

Figure 8 shows the simulated spatial growth of the ion-acoustic waves. The calculated amplitude profile is shown in Fig. 9 after post-processing the simulation and averaging over a $70 \mu \mathrm{m}$ area similar to the experiments. It is evident that large ion-acoustic waves are driven only in the front half of the plasma. The velocity gradient in the back of the plasma prevents any significant SBS growth. The plateau in the middle provides a resonant region where SBS drives a large ion-acoustic wave. In the front of the plasma, the strong velocity gradient prevents SBS growth at the local resonant frequency, but the off-resonant ponderomotive force drives non-resonant acoustic waves in the front of the plasma (Fig. 8).

While the amplitude of the driven acoustic waves $\left(\frac{\delta n}{n}\right)$ is readily obtained from simulations, it has to be calculated from the measured intensity peaks in the Thomsonscattering spectra. This is done by comparing the scattered reflectivity from thermal fluctuations (i.e., the power scattered into the left intensity peak in Fig. 2a divided by the incident laser power), which is given by:

$$
R_{\text {thermal }}=\frac{1}{4} r_{0}^{2} n_{e} L_{3 \omega} \Delta \Omega_{\text {exp }}
$$

where $r_{0}$ is the classical electron radius, $L_{3 \omega}$ is the length along the direction of the $3 \omega$ probe beam, and $\Delta \Omega_{e x p}$ is the solid angle of the collecting optics (an $\mathrm{f} / 5$ lens), with the reflectivity scattered by the driven acoustic wave given by:

$$
R_{\text {driven }}=\frac{1}{4} r_{0}^{2} n_{e}^{2} \lambda_{3 \omega}^{2} L_{3 \omega} L_{c}\left(\frac{\delta n}{n_{e}}\right)^{2}
$$

where $\delta n / n_{\leftarrow}$ is the amplitude of the acoustic wave and $L_{c}$ its correlation length along the direction of the $3 \omega$ probe heam. $T_{c}$ can be estimated by the transverse size of speckles generated by the interaction beam $f_{2 \omega} \lambda_{2 \omega}$.

Figure 9 shows that this estimation of $\delta n / n_{e}$ reproduces the overall spatial shape predicted by $\mathrm{pF} 3 \mathrm{~d}$, but the absolute amplitude is smaller by almost an order of magnitude. Various factors can explain this discrepancy. For instance, any misalignment of the optics, an overestimation of $L_{c}$, or enhanced thermal fluctuations would lead to an underestimation of the acoustic wave amplitude. Assuming the latter, we have verified that increasing the amplitude of the thermal noise source used both in the Thomson-scattering calculations and in the SBS simulations by an order of magnitude gives a good agreement between the measured and simulated wave amplitude and spatial profiles. A study of the amplitude of the thermal noise in laser-induced plasmas needs to be completed to investigate the discrepancies in the calculated wave amplitudes seen in this and other studies [16].

Figure 10a plots the average SBS reflectivity as a function of the length of the plasma along the interaction

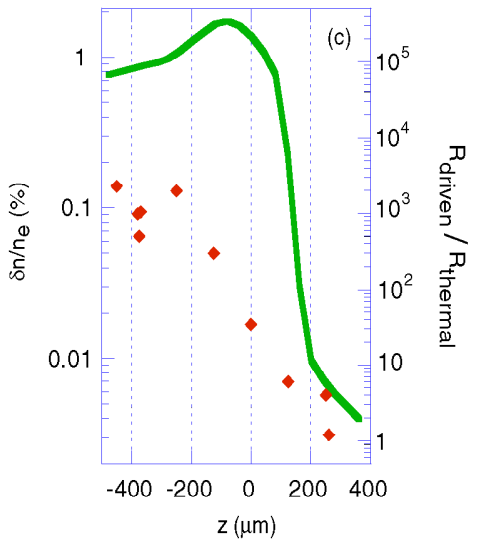

FIG. 9: The measured amplitude of the excited ion-acoustic wave $\left(\frac{\delta n}{n_{c}}\right)$ is plotted as a function of the position along the interaction beam. The calculated amplitude profile from $\mathrm{pF} 3 \mathrm{~d}$ (solid line) after averaging over a $70 \mu \mathrm{m}$ area similar to the experiment is shown.

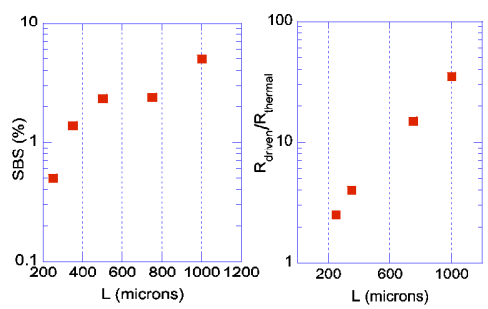

FIG. 10: The (a) SBS reflectivity and (b) normalized ionwave amplitude are plotted as a function of plasma length along the interaction beam axis.

beam $(\mathrm{L})$. This scaling was done by using various sizes of targets. The SBS reflectivity should scale exponentially with the length of the velocity plateau. From simulations it is apparent that there is no velocity plateau for targets shorter than 600 microns. Therefore, the reflectivity below this point is due to scattering from regions of the plasma where there is a velocity gradient. In these cases, the reflectivity is very sensitive to the actual shape of the velocity gradient; and no simple scaling is available. As shown previously, a very precise characterization of the plasma conditions was necessary to model SBS from the largest 1-mm targets (see Sec. IV A). Figure 10b shows the measured ion-acoustic wave ratio $\left(\mathbf{R}_{\text {driven }} / R_{\text {thermal }}\right)$ in the middle of the plasma $(z=0)$ as a function of the plasma length along the interaction beam $(\mathrm{L})$. 


\section{B. Kinetic effects (Trapping)}

When the intensity exceeds $\mathrm{I}>1.5 \times 10^{15} \mathrm{~W} \mathrm{~cm}^{-2}$, it is clear that even with a strong velocity gradient non-linear kinetic effects are needed to explain saturation of the SBS instability. In our range of experimental parameters $\left(6<Z_{B e} T_{e} / T_{i}<14\right)$ there are many particles at the ion-acoustic phase velocity; therefore, non-linear kinetic effects are a plausible mechanism to saturate SBS

By adding small amounts of Au to our Be plasmas, we were able to accurately measure an increase in ion temperature when ion-acoustic waves were driven to large amplitude. For these plasmas, the solution to the kinetic dispersion relation reveals two modes which react qualitatively differently when the ion temperature is changed. Namely, when the ion temperature is increased, the damping of the two modes move inversely. Figure 11a shows the multi-ion spectra for two different ion temperatures. These data are fit using a multi-ion form factor with two free sensitive fit parameters $\left(\mathrm{T}_{i}, \mathrm{~T}_{e}\right)$ [17]. The frequency shift of the outter peak is set by the electron temperature, while the relative amplitude of the two intensity peaks is determined by the ion temperature.

Figure 11b shows the ion temperature increase as a function of the SBS excited ion-acoustic wave amplitude. As the interaction beam excites ion-acoustic waves, the ion temperature in the plasma was measured to increase, while we found no change in the electron temperature. Comparing Fig. 7 and Fig. 11b it is evident that the ncrease in ion temperature is directly linked to the saturated regime. This direct observation of an increase in ion temperature is an indication of ion trapping. In this experiment ion-acoustic waves are excited in the direction of the interaction beam. These driven acoustic waves trap ions which are then accelerated creating hot ions. $\Lambda \mathrm{ftcr}$ thermalization this will increase the average ion temperature which we have measured using Thomson scattering.

Trapping induces a modification of the dispersion relation for the SBS acoustic wave, creating a non-linear frequency shift $[25,27,33]$. This non-linear frequency shift detunes the SBS resonance therefore, saturating the ion-acoustic wave. Using this trapping model, the calculated SBS reflectivity is close to the $5 \%$ measured in our experiments [15].

Furthermore, we have tested this model by estimating the ion temperature in the Thomson-scattering volume. We balance the energy flux deposited into the ionacoustic waves (from the Manley-Rowe relations) with a free streaming heat flux [8]. This model assumes that the energy deposition is local and thermalization is fast enough so that heat is carried away by Maxwellian ions when a steady state is reached. Theoretical and experimental ion-wave amplitudes were related by matching a high reflectivity point; the model then reproduces the experimental scaling for ion temperature (Fig. 11b)

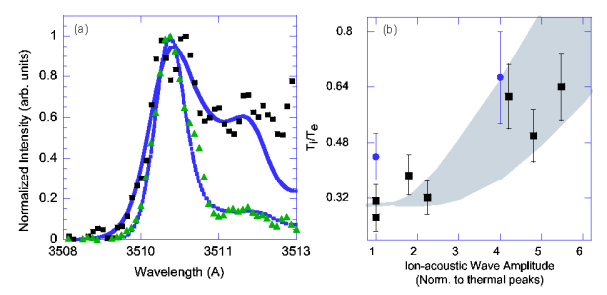

FIG. 11: (a) The blue-shifted (thermal) part of the Thomsonscattering spectra for a $10 \%$ A 11 mixtıre are shown for a shot without the interaction beam (triangels) and a shot with an interaction beam intensity of $7 \times 10^{15} \mathrm{~W}_{-} \mathrm{cm}^{-2}$ (squares) resulting in an ion temperature from $250 \mathrm{eV}$ to $475 \mathrm{eV}$. 0(b) The ratio of $T_{e} / T_{i}$ as a function of the normalized ion-wave amplitude shows evidence of the generation of hot ions and ion trapping. The ion-wave amplitude is normalized to the thermal ion-wave amplitude. Data for Be plasmas with $5 \%$ Au (circles) and $10 \% \mathrm{Au}$ (squares) show a factor of two increase in ion temperature. The gray area represents calculated results from a simple energy balance between energy deposition due to SBS and a free-streaming heat flux.

\section{SUMMARY AND CONCLUSIONS}

We have presented a detailed study of SBS saturation mechanisms in low-Z Be plasmas. We find that detuning of the SBS instability by velocity gradients and by trapping are effective mechanisms for saturating the SBS reflectivity. Direct evidence of detuning of SBS by a velocity gradient was observed through a novel use of two Thomson-scattering diagnostics, allowing us to directly measure the frequency shift between the frequency of the SBS driven acoustic wave relative to the local resonant acoustic frequency. Furthermore, we have shown that adding small amounts of Au to our Be plasmas has allowed us to accurately determine the ion temperature by measuring the relative damping of two ion-acoustic modes. We report up to a factor of two increase in ion temperature when ion-acoustic waves are excited by SBS.

This increase in ion temperature is a strong indication of hot ions due to trapping.

Trapping is an efficient saturation mechanism in low$\mathrm{Z}$ plasmas because there are significant particles at the waves phase velocity, but in general trapping does not explain saturation in high- $\mathrm{Z}$ plasmas. Both the ionacoustic wave and SBS reflectivity have experimentally been shown to saturate in high-Z plasmas [16]. Previous non-linear saturation mechanisms such as two-ion-decay or harmonic generation in high-Z plasmas have been suggested, but these effects have never been experimentally or theoretically verified.

In part due to the measured kinetic effects, trapping is currently being implemented in models to help simulate low-Z conditions [34], and experiments are underway to investigate saturation mechanisms in high $\mathrm{Z}$ plasmas. 
Understanding the saturation mechanisms in high-Z plasmas is critical in the development of indirect-drive inertial confinement fusion

\section{ACKNOWLEDGMENTS}

We would like to acknowledge the efforts of the Trident laser crew: T. Hurry, R. Gonzales, N. Okamoto, F.
Archuleta, and R. Gibson. We appreciate the effort of R. Pera in target fabrication at LANL. We thank R. Scalettar, J. Fernandez, A. Hauer, and the UC Davis Physics Department for their support.

This work is partially supported by LDRD 01-ERD107 and was performed under the auspices of the U.S. Department of Energy by the Lawrence Livermore National Laboratory under Contract No. W-7405-ENG-48.
[1] R. L. Berger, C. Still, E. A. Williams, and A. B. Langdon, Phys Plasmas 5, 4337 (1998)

[2] H. A. Rose and D. F. DuBois, Phys Rev Lett 72, 2883 (1994).

[3] C. E. Clayton, C. Joshi, and F. F. Chen, Phys Rev Lett 51, 1656 (1983).

[4] B. Gellert and B. Kronast, Appl Phys B 32, 175 (1983).

[5] S. C. Wilks, W. L. Kruer, J. Denavit, K. G. Estabrook, D. E. Hinkel, D. Kalantar, A. B. Langdon, B. MacGowan, D. S. Montgomery, and E. A. Williams, Phys Rev Lett 74, 5048 (1995).

[6] S. H. Glenzer, L. M. Divol, R. L. Berger, C. Geddes, R. K. Kirkwood, J. Moody, E. A. Williams, and P. E. Young, Phys Rev Lett 86, 2565 (2001).

[7] M. Herbst, C. E. Clayton, and F. F. Chen, Phys Rev Lett 43, 1591 (1979).

[8] W. L. Kruer, Phys Fluids 23, 1273 (1980)

[9] J. Handke, S. A. Rizvi, and B. Kronast, Phys Rev Lett 51, 1660 (1983).

[10] J. E. Bernard and J. Meyer, Phys Rev Lett 55 , 79 (1985).

[11] C. S. Lui, M. N. Rosenbluth, and R. B. White, Phys Rev Lett 31 (1973).

[12] D. H. Froula, L. M. Divol, and S. H. Glenzer, Phys Rev Lett 88, 105003 (2002).

[13] N. K. Moncur, R. P. Johnson, R. G. Watt, and R. B. Gibson, Appl Opt 34, 4274 (1995).

[14] B. S. Bauer, R. P. Drake, K. G. Estabrook, R. G. Watt, M. Wilke, and S. A. Baker, Phys Rev Lett 74,3604 (1995)

[15] D. H. Froula, L. Divol, H. A. Baldis, R. L. Berger, D. G. Braun, B. I. Cohen, R. P. Johnson, D. S. Montgomery, E. A. Williams, and S. H. Glenzer, Phys Plasmas 9, 4709 (2002).

[16] S. H. Glenzer, C. A. Back, K. G. Estabrook, R. Wallace, K. Baker, B. J. MacGowan, B. A. Hammel, R. E. Cid, and J. S. D. Groot, Phys Rev Lett 77, 1496 (1996).

[17] D. E. Evans, Plasma Phys 12, 573 (1970).
[18] D. H. Froula, Ph.D. thesis, University of California at Davis (2002)

[19] J. A. Fejer, Can J Phys 38, 1114 (1960)

[20] G. B. Zimmerman and W. L. Kruer, 2, 51 (1975).

[21] J. C. Fernandez, J. A. Cobble, D. S. Montgomery, M. D. Wilke, and B. B. Afeyan, Phys Plasmas 7, 3743 (2000).

[22] S. H. Glenzer, L. J. Suter, R. L. Berger, K. G. Estabrook, B. A. Hammel, R. L. Kauffman, R. K. Kirkwood, B. J. MacGowan, J. D. Moody, J. E. Rothenberg, et al., Phys Plasmas 7 (2000).

[23] J. D. Moody, B. J. MacGowan, J. E. Rothenberg, R. L. Berger, L. Divol, S. H. Glenzer, R. K. Kirkwood, E. A. Williams, and P. E. Young, Phys Rev Lett 86, 2810 (2001).

[24] R. K. Kirkwood, B. J. MacGowan, D. S. Montgomery, B. B. Afeyan, W. L. Kruer, D. M. Pennington, S. C. Wilks, J. D. Moody, K. Wharton, C. A. Back, et al., Phys Plasmas 4, 1800 (1997).

[25] A. A. Andreev and V. T. Tikhonchuk, JETP 68, 1135 (1989).

[26] W. L. Kruer, From fusion to light surfing (Addison-Wesley, Redwood City, CA, 1991).

[27] B. I. Cohen, B. F. Lasinski, A. B. Langdon, and E. A. Williams, Phys Plasmas 4, 956 (1997).

[28] C. Riconda, Physica Scripta 84, 217 (2000).

[29] C. J. Pawley, H. E. Huey, and N. C. J. Luhmann, Phys Rev Lett 49, 877 (1982).

[30] P. W. Rambo, S. C. Wilks, and W. L. Kruer, Phys Rev Lett 79, 83 (1997).

[31] J. A. Heikkinen, S. J. Karttunen, and R. R. E. Salomaa, Phys Fluids 27, 707 (1984).

[32] W. Rozmus, Phys Fluids B4, 576 (1992).

[33] G. J. Morales and T. M. O'Neil, Phys Rev Lett 28, 417 (1972).

[31] R. K. Kirkwood, J. D. Moody, A. B. Langdon, B. I. Cohen, E. A. Williams, M. R. Dorr, J. A. Hittinger, R. L. Berger, P. E. Young, L. J. Suter, et al., Phys Rev Lett 89, 215003 (2002). 
Appendix D

"Direct observation of stimulated

Brillouin scattering (SBS)

detuning by a velocity gradient",

D. H. Froula, et al., Physical

Review Letters, submitted (2002) 


\title{
Direct observation of stimulated Brillouin scattering (SBS) detuning by a velocity gradient
}

\author{
D. H. Froula, ${ }^{*}$ L. Divol, A. MacKinnon, G. Gregori, and S. H. Glenzer \\ L-399, Lawrence Livermore National Laboratory
}

University of California P. O. Box 808, CA 94551, U.S.A.

\begin{abstract}
We report the first direct evidence of detuning of stimulated Brillouin scattering (SBS) by a velocity gradient, which was achieved by directly measuring the frequency shift of the SBS driven acoustic wave relative to the local resonant acoustic frequency. We show that in the expanding part of the plasma, ion-acoustic waves are driven off-resonance which leads to the saturation of the SBS instability. These measurements are well reproduced by fluid simulations that include the measured flow.
\end{abstract}

PACS numbers: $52.25 . \mathrm{Qt}, 52.35 . \mathrm{Fp}, 52.40 . \mathrm{Nk}, 52.50 . \mathrm{Jm}$

Stimulated Brillouin scaltering (SBS) is the result of the resonant ponderomotive coupling of an incident light wave, a reflected light wave, and an ion-acouslic wave. This resonance can drive ion-acoustic waves to large amplitudes. In principle, this process could result in the reflection of a large fraction of the incident energy for inertial confinement fusion (ICF) targets. In order to minimize this energy loss, a good understanding of mechanisms that could limit SBS is important. In many targets, a velocity gradient within the expanding plasma provides such a mechanism; the frequency of the local acoustic waves is Doppler shifted, therefore, detuning the three-wave resonant process [1]

In this letter, we present the first direct measurement of SBS detuning by a velocily gradient. A novel use of two Thomson-scattering diagnostics has allowed us to directly measure the frequency and amplitude of the ion-acoustic wave responsible for SBS as a function of space. This is the first measurement that spatially resolves both the frequency and the amplitude of the ionacoustic waves directly responsible for SBS. These medsurements link the saturation of the SBS instability to the frequency detuning from an expanding plasma. We have independently measured the ion-acoustic frequency and the frequency of the driven acoustic wave at various positions in the plasma. Therefore, by comparing the local ion-acoustic frequency with the local frequency of the driven acoustic wave we have measured the actual detuning of the SBS instability. The measured electron temperature, velocity gradient, and electron density have been included in fluid simulations which clearly support the measured SBS detuning by a velocity gradient.

The experiments used a three-beam configuration at the Trident Laser Facility [2]. The Be plasmas were produced by a preform plasma beam with $180 \mathrm{~J}$ of $2 \omega$ $(\lambda=527 \mathrm{~nm})$ laser light in a 1.2 -ns-long square pulse. The preform plasma beam was focused normal to the target surface using an $f / 6$ lens and a strip line random phase plate (Fig. 1c). This produced a line focus with an intensity of $10^{14} \mathrm{~W} \mathrm{~cm}^{-2}$ and an initial $1000 \mu \mathrm{m} \times 100 \mu \mathrm{m}$ Be plasma [3]. An interaction

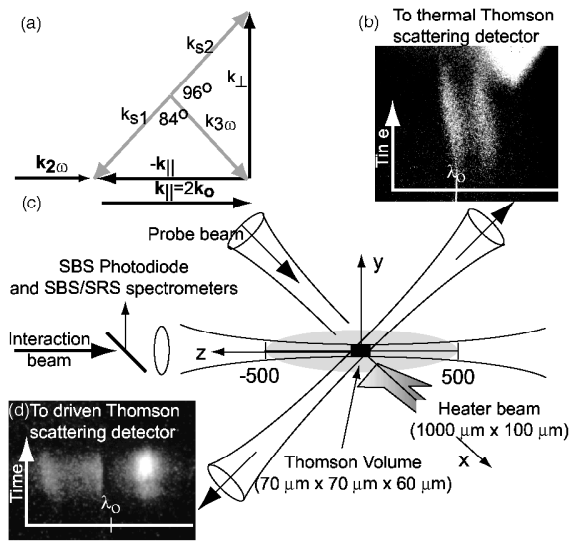

FIG. 1: (a) A diagram detailing the ion-acoustic wave-vectors probed is shown. (b) A thermal Thomson-scattering spectrum $\left(\mathrm{k}_{\perp}\right)$ and (d) a driven Thomson scattering spectrum $\left(\mathrm{k}_{\mid}\right)$are shown. (c) The experimental setup is shown.

beam ( $50 \mathrm{~J}, 2 \omega, 1.2$-ns-long square pulse) was aligned $\mathrm{x}=400 \mu \mathrm{m}$ from the target surface and was focused to a $60 \mu \mathrm{m}$ diameter spot, resulting in an intensity of $1.5 \times 10^{15} \mathrm{~W} \mathrm{~cm}^{-2}$. The interaction beam was used to drive SBS which excites ion-acoustic waves with wavevector $\mathbf{k}_{1}=2 \mathbf{k}_{\mathbf{o}}$ co-propagating in the direction of the interaction beam. The backscattered SBS light was collected and collimated by the focusing lens of the interaction bcam. $\Lambda$ fraction of the backscattcred light was focused on to a fast photodiode and two, 1/4-meter SRS and 1-meter SBS, spectrometers. The SRS and SBS spectra were measured with a respective resolution of $0.5 \mathrm{~nm}$ and $0.45 \AA$. The absolute SBS cnergy was moasured with the diode [4].

The third laser beam, $3 \omega(\lambda=3513 \AA)$, was used as a Thomson-scattering probe beam. The probe beam and Thomson-scattering collection optics define 


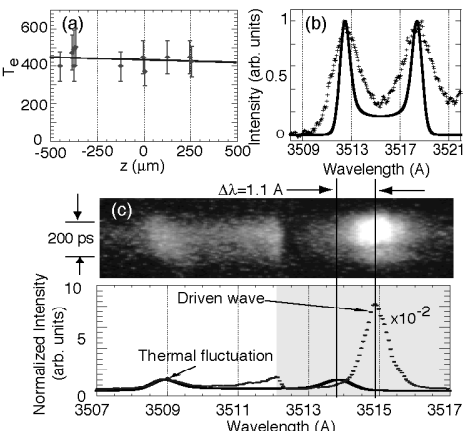

FIG. 2: (a) A constant electron temperature, $T_{0}=450 \mathrm{eV}$, is measured along the interaction beam axis. The Be is fully ionized $(Z=4)$. (b) Representative Thomson-scattering spectra taken from the spectra in Fig. 1b (points) where light is scattered from ion-acoustic fluctuations propagating perpendicular to the interaction beam $\left(\mathbf{k}_{\perp}\right)$; the theoretical form factor is fit (line). (c) Thomson-scattering data are shown collected by the diagnostic observing ion acoustic waves propagating parallel to the interaction beam $\left(\mathbf{k}_{\mid}\right)$. The spectra (points) is shown below averaged over 50 ps. The theoretical form fac tor (solid line) is fit to the blue-shifted light using a velocity flow $\frac{v}{v}=0.7$ and the electron temperature measured with the first Thomson-scattering diagnostic $\left(T_{e}=450 \mathrm{eV}\right)$. The shaded region was optically filtered by x100

a volume $(70 \mu \mathrm{m} \times 70 \mu \mathrm{m} \times 60 \mu \mathrm{m})$ located at the center of the chamber. Light scattered from the Thomson-scattering volume was imaged onto two separate Thomson-scattering spectrometers. The interaction beam is focused in the middle of this volume. The first Thomson-scattering diagnostic probes ion-acoustic fluctuations (Fig. 1b) propagating perpendicular to the interaction beam $\left(\mathbf{k}=\mathbf{k}_{\perp}\right)$ and allows us to independently measure the electron temperature, $T_{e}$. The geometry (Fig. 1a) of the second Thomson-scattering diagnostic was chosen to measure light scattered from SBS-driven ion-acoustic waves (Fig. 1d) that are excited by the SBS instability $\left(\mathbf{k}=\mathbf{k}_{\mid}\right)[5]$.

The interaction beam was timed to turn on $1.0 \mathrm{~ns}$ after the preform plasma beam. The 180 ps Gaussian Thomson probe beam was turned on 400 ps after the interaction beam near the peak of the backscattered SBS light

The plasma has been well-characterized; Fig. 2 a shows a constant clcctron temperature profile $\left(\mathrm{T}_{e}=450 \mathrm{cV}\right.$ ) along the interaction beam axis. The location of the Thomson-scattering volume and the focus of the interaction beam remained at TCC throughout the experiment; the target (and the preformed plasma beam) was moved to probe different positions in the plasma parallel to, and $400 \mu \mathrm{m}$ from, the surface of the target.
Figure $2 \mathrm{~b}$ shows a Thomson-scattering spectrum taken from the streak data in Fig. 1b. Two pcaks corresponding to ion-acoustic fluctuations propagating along the direction of $\mathbf{k} \perp$ are evident. Fitting the data using a standard theoretical form factor gives the electron temperature [6] . The measured peaks are broadened by ve locity gradients perpendicular to the interaction beam. This broadening is to be expected as the initial plasma length is only $\mathrm{y}=100 \mu \mathrm{m}$ in the perpendicular direction; this Thomson scattering diagnostic probes the plasma expanding in the perpendicular direction. The measured width of the peaks $(\delta \lambda)$ gives an estimated velocity range $\frac{\delta v}{v}\left(\propto \frac{\delta \lambda}{\lambda}\right)=0.5$ within the Thomson-scattering volume. The instrument function of the spectrometer was measured to be $0.2 \AA$.

The blue-shifted intensity peak in the spectra mesured by the second Thomson-scattering diagnostic is a direct measure of the local resonant frequency of the ionacoustic waves in the laboratory frame $(-\mathbf{k} \mid)$. The frequency of the scattered light in the laboratory frame is shifted from the laser frequency in two ways: by the frequency of the probed ion-acoustic wave $\left(\omega-c_{s} k_{4}\right.$, where $c_{S}$ is the sound speed which is a function of the electron temperature), and by the overall flow, $\mathbf{u}_{\mathbf{f}}$, of the plasma relative to the frame of the laboratory (Doppler shift $\mathbf{k}_{||} \cdot \mathbf{u}_{\mathbf{r}}$ ) which leads to a wavelength shift in the Thomson-scattered light given by,

$$
\Delta \lambda=\frac{\lambda_{3 \omega}^{2}}{2 \pi c} \mathbf{k}_{||} \cdot\left(\mathbf{c}_{\mathbf{s}}+\mathbf{u}_{\mathbf{f}}\right)
$$

Since the electron temperature is known, the plasma flow parallel to the $\mathrm{z}$-axis is determined from the local frequency shift between the frequency of the Thomsonscattering probe $\left(\lambda_{3 \omega}=3513 \AA\right)$ and the blue-shifted intensity peak.

Figure 2c shows a Thomson-scattering spectra in which two intensity peaks can be observed. The blue-shifted intensity peak is fit using the independently measured electron temperature, and a flow of $\frac{v}{c_{s}}=0.7$ is measured. Note that the use of two Thomson-scattering diagnostics is necessary as the SBS-driven acoustic wave is not always at the local resonant ion-acoustic frequency. This is evident in Fig. 2c, where the red-shifted intensity peak and the corresponding resonant intensity peak are offset by $1.1 \AA$.

Figure 3d shows the plasma flow along the z-axis measured from the frequency of the local resonant ionacoustic fluctuations $\left(\mathbf{k}_{||}\right) . \Lambda 200 \mu \mathrm{m}$ velocity plateau is measured in the center of the plasma; a Mach 1 flow was measured in the front edge of the plasma. One can directly observe the effect of the velocity gradient on SBS from the froqueney mismatch between the measured frequency of the SBS driven acoustic wave, and the measured frequency of the local resonant waves.

Figure 3 shows spectra for representative data shots at three positions in the plasma; the frequency of the driven 

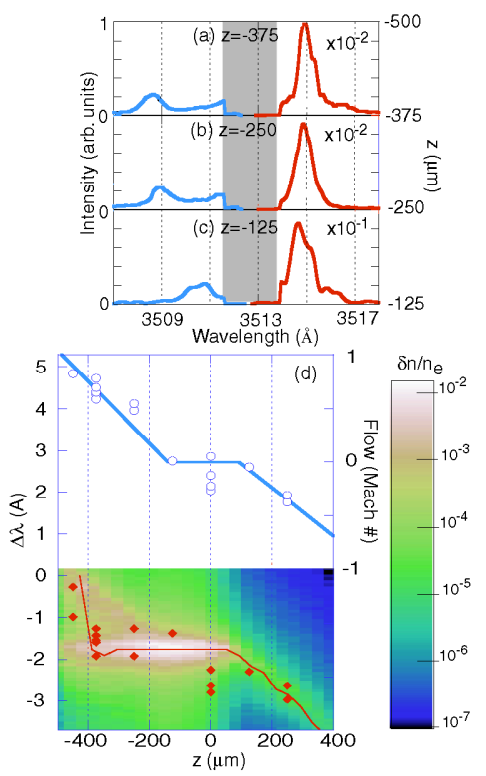

FIG. 3: 'I'homson-scattering spectra at $\mathrm{z}=$ (a) $-375 \mu \mathrm{m}$, (b) $-250 \mu \mathrm{m}$, and (c) $-125 \mu \mathrm{m}$ are shown. The increasing shift of the thermal peak (blue peaks) indicates a velocity flow. One can see that the frequency of the SBS-driven ion-acoustic wave remains unchanged (red peaks). The shaded area has been filtered by a factor of 10 . (d) The wavelength shift for the two peaks are plotted for every Thomson-scattering spectra obtained. The wavelength shift of the red-shifted peaks (red points) reveals off-resonant ion-acoustic waves in the front of the plasma. A pF3d simulation is shown (color) where large acoustic waves are driven in the plateau region and the frequency of the driven acoustic waves remain constant in the front of the plasma (red line)

ion-acoustic wave remains unchanged $(\lambda=3515 \AA$ ) while the frequency of the local thermal ion-acoustic waves $\left(-\mathbf{k}_{\|}\right)$increase as one moves from the plateau to the front of the plasma (this increase is a direct effect of the plasma flow as the electron temperature is constant). SBS light is scattered from the velocity plateau where large resonant acoustic waves grow exponentially (shadded region Fig. 4c). The light scattered from the plateau and the incident light create a constant ponderomotive force with a constant frequency profile in the front of the plasma. This constant frequency ponderomotive force drives nonresonant acoustic waves (i.e., the velocity gradient shifts the resonant frequency from the constant frequency of the ponderomotive force)

The experiment was simulated in $2 \mathrm{D}$ using pF3d [7]. A $f / 6$ RPP beam with a best focus of $60 \mu \mathrm{m}$ and an average vacuum intensity of $10^{15} \mathrm{~W} \mathrm{~cm}^{-2}$ was propa

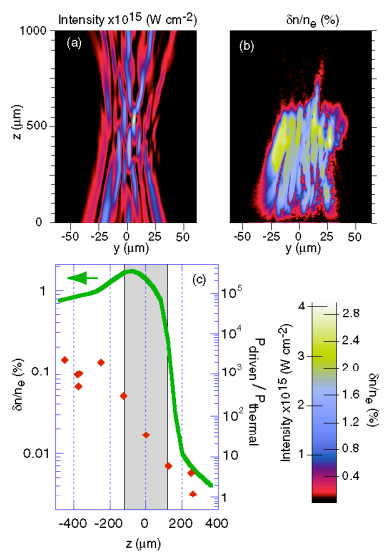

FIG. 4: (a) The intensity of the interaction beam is plotted $70 \mathrm{ps}$ into the pF3d simulation. (b) The ion-acoustic waves are large in the front of the plasma. (c) The measured amplitude of the excited ion-acoustic wave (diamonds) is plotted as a function of the position along the interaction beam. The amplitude profile given by $\mathrm{pF} 3 \mathrm{~d}$ is also plotted (solid). The shaded region represents the velocity plateau.

gated through a $1000 \mu \mathrm{m}$ slab of plasma $\left(T_{e}=450 \mathrm{eV}\right.$, $n_{e}=1 \times 10^{20} \mathrm{~cm}^{-3}$ ). A Mach 2 transverse flow (lateral expansion of the target) and the measured velocity profile along the propagation axis shown in Fig. 3d were included.

Figure $4 \mathrm{a}$ shows a plot of the interaction beam $70 \mathrm{ps}$ into the simulation. Intensity of the most intense speckles is around $4 \times 10^{15} \mathrm{~W} \mathrm{~cm}^{-2}$. A linear non-local heat transport model was used, but had only a small effect on the results. The maximum local temperature fluctuation observed was $\frac{\Delta T}{T} \approx 30 \%$ in the most intense speckles. The transverse flow suppressed most of the filamentation and the simulations show little beam bending (Fig. 4a).

Figure $4 \mathrm{~b}$ and the solid line in Fig. 4c show that large ion-acoustic waves are driven by SBS only in the front half of the plasma [8]. The velocity gradient in the back of the plasma prevents any significant SBS growth. The plateau in the middle provides a resonant region where SBS drives ion-acoustic waves exponencially to large amplitude. In the front of the plasma, the strong velocity gradient prevents SBS growth at the local resonant frequency, but the reflected light coming from the plateau and the incoming light provide a constant frequency ponderomotive force that drives non-resonant acoustic waves as secn between $\mathrm{z}=200 \mu \mathrm{m}$ and $\mathrm{z}=400 \mu \mathrm{m}$ in Fig. 3 . As one moves away from the plateau and towards the front of the plasma, the acoustic wave amplitude slightly decreases as the ponderomotive driver is increasingly offresonance (Fig. 4c). This effect of detuning SBS by a 
velocity gradient is further verified by post processing the simulation providing a measure of the local frequeney of the driven ion-acoustic waves. This agrees well with the expcrimentally measured frequencics, as shown in Fig. 3d; off-resonant driven ion-acoustic waves are evident in the front part of the plasma.

While the amplitude of driven acoustic waves $\left(\frac{\delta n}{n_{n}}\right)$ is readily obtained from simulations, it has to be calculated from the intensity peaks in the Thomson-scattering spectra. This is done by comparing the scattered power from thermal fluctuations (i.e., the power scattered in to the left intensity peak in Fig. 2c), with the power scattered by the driven acoustic wave which gives the ratio,

$$
\frac{P_{\text {driven }}}{P_{\text {thermal }}}=n_{e} \lambda_{3 \omega}^{2} \Delta \Omega_{e x p} L_{c}\left(\frac{\delta n}{n_{e}}\right)^{2}
$$

where $\delta n / n_{e}$ is the amplitude of the acoustic wave, $L_{c}$ its correlation length along the direction of the $3 \omega$ probe beam, and $\Delta \Omega_{\exp }$ is the solid angle of the collectiong optics (an $f / 5$ lens). $L_{c}$ can be estimated by the transverse size of speckles generated by the interaction beam $f_{2 \omega} \lambda_{2 \omega}$.

Figure $4 \mathrm{c}$ shows that the estimation of $\delta n / n_{e}$ from Eq. 1 reproduces the overall spatial shape predicted by pF3d, but the absolute amplitude is smaller by almost an order of magnitude. Various factors can explain this discrepancy. For instance, any misalignment of the optics, an overestimation of $L_{c}$, or enhanced thermal fluctuations would lead to an underestimation of the acoustic wave amplitude. Assuming the latter, we have verified that increasing the amplitude of the thermal noise source used both in the Thomson-scattering calculations and in the SBS simulations by an order of magnitude gives a good agreement between the measured and simulated wave amplitude and spatial profiles. A study of the amplitude of the thermal noise in laser plasmas needs to be completed to investigate the discrepancies in the calculated wave amplitudes seen in this and other studies [9].

A peak SBS reflectivity of $6 \%$ was measured. The SBS spectrum (Fig. 5) confirms our analysis of the effect of the velocity gradient on SBS. The frequency of the peak intensity corresponds to the resonant acoustic frequency in the velocity plateau. The spectrum has a tail towards the fundamental frequency that corresponds to light scattered from the flow propagating towards the detector There is a sharp cutoff towards higher wavelengths indicating that there is no scattering coming from the back of the plasma where the velocity flow is propagating away from the detector. This is consistent with the fact that we measure very small ion-acoustic waves in the back portion of the plasma (Fig. 4c). Overall, the SBS spectrum is characteristic of the central plateau, while the expanding plasma only slightly modifies its shape.

In summary, we have presented the first direct measurement of SBS detuning by a velocity gradient. We

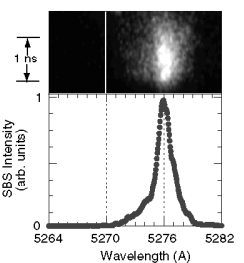

FIC. 5: Ths SBS spectra is plotted as a function of intensity. The peak intensity corresponds to light scattered from the velocity plateau; a tail towards the fundamental frequency corresponds to light scattered from ion-acoustic waves in the front of the plasma.

studied an expanding plasma with a central velocity plateau. The frequency mismatch between the frequency of the SBS driven ion-acoustic wave and the local resonant frequency (as measured from thermal waves) was mapped throughout the plasma. It was found that acoustic waves in the flow are driven off-resonance by the ponderomotive force with a beat frequency created by the incoming light and the light reflected from the plateau. This detuning inhibits further SBS growth in the expanding plasma. These findings are important to many laserplasma experiments and occurs in various ICF directdrive $[10]$ and indirect-drive targets $[9]$. We have shown that the SBS spectrum gives insight into the locations of SBS growth, and Thomson scattering on driven ionacoustic waves provides a direct verification of the effect of a velocity gradient on SBS.

We would like to acknowledge the efforts of the Trident laser crew. We thank D. Montgomery and H. Baldis for valuable discussions. This work was performed under the auspices of the U.S. Department of Energy by the Lawrence Livermore National Laboratory under Contract No. W-7405-ENG-48.

* Also at Physics Department, University of California at Davis, 95616 .

1] C. S. Lui et al., Physical Review Letters 31 (1973).

[2] N. K. Moncur et al., Applied Optics 34, 4274 (1995).

[3] B. S. Baner et al., Physical Review Tetters 74, 3604 (1995).

4] D. H. Froula et al., Physics of Plasmas 9, 4709 (2002).

[5] D. H. Froula et al., Physical Review Letters 88, 105003 (2002).

[6] D. E. Evans, Plasma Physics 12, 573 (1970)

[7] R. L. Berger et al., Physics of Plasmas 5, 4337 (1998).

[8] J. A. Cobble et al., Physics of Plasmas 7, 323 (2000).

[9] S. H. Glenzer et al., Physical Review Letters 77, 1496 (1996).

[10] W. Seka et al., Physical Review Letters 89, 175002 (2002). 